\title{
DEVELOPMENT OF A KINEMATIC HARDENING RULE TO ASSESS RATCHETING RESPONSE OF MATERIALS UNDER VARIOUS MULTIAXIAL LOADING SPECTRA
}

\author{
By \\ SeyedMahdi Hamidinejad \\ B.Sc. in Mechanical Engineering, Najafabad Azad University, Iran, 2006 \\ M.Sc. in Mechanical Engineering, Ferdowsi University of Mashhad, Iran, 2009
}

\begin{abstract}
A dissertation
presented to Ryerson University

in partial fulfillment of the

requirements for the degree of
\end{abstract}

\section{Doctor of Philosophy}

in the Program of

Mechanical Engineering

Toronto, Ontario, Canada, 2015

(C) SeyedMahdi Hamidinejad 2015 


\section{AUTHOR'S DECLARATION FOR ELECTRONIC SUBMISSION OF A DISSERTATION}

I hereby declare that I am the sole author of this dissertation. This is a true copy of the dissertation, including any required final revisions, as accepted by my examiners.

I authorize Ryerson University to lend this dissertation to other institutions or individuals for the purpose of scholarly research.

I further authorize Ryerson University to reproduce this dissertation by photocopying or by other means, in total or in part, at the request of other institutions or individuals for the purpose of scholarly research.

I understand that my dissertation may be made electronically available to the public. 


\title{
ABSTRACT \\ Development of A Kinematic Hardening Rule to Assess Ratcheting Response of Materials under various Multiaxial Loading Spectra
}

\author{
SeyedMahdi Hamidinejad, Doctor of Philosophy in Mechanical Engineering, Ryerson \\ University, Toronto, Canada, 2015
}

The present thesis develops an Armstrong-Frederick (A-F) type coupled kinematic hardening rule to assess ratcheting response of steel alloys under various multiaxial loading paths. The hardening rule is constructed on the basis of the recently proposed Ahmadzadeh-Varvani (AV) hardening rule to further evaluate the ratcheting response of materials under multiaxial loading spectra. The modified model offers a simple framework with limited number of terms and coefficients in the dynamic recovery portion of the model. The dynamic recovery further holds inner product of plastic strain increment $d \bar{\varepsilon}_{p}$ and backstress unit vector $\bar{a} /|\bar{a}|$ with different directions under multiaxial stress cycles enables the model to track different directions. Term $\langle\bar{n} . \bar{a} /|\bar{a}|\rangle^{1 / 2}$ taking positive values less than unity for multiaxial loading conditions is to control the accumulation rate of ratcheting strain and to prevent the modified model to experience plastic shakedown over stress cycles in stage II. Term $(2-\bar{n} \cdot \bar{a} /|\bar{a}|)$ taking the values between 1 and 3 under multiaxial loading, magnifies the effect of coefficient $\gamma_{2}$ to take into account the nonproportionality effect of various loading paths and further to shift down the predicted ratcheting strain over the stress cycles.

The predicted ratcheting curves by the modified rule were compared with those predicted based on earlier developed hardening rules of Ohno-Wang (O-W), Jiang-Sehitoglu (J-S), McDowell, and Chen-Jiao-Kim (C-J-K) holding relatively complex framework and more number of coefficients. The O-W, the J-S, McDowell and C-J-K models mainly deviated from the experimental ratcheting strain of steel alloys for various multiaxial loading histories, while the 
predicted curves of the modified model closely agreed with experimental data of steel samples over ratcheting stages. The predicted ratcheting curves based on the modified model closely agreed with experimental data of steel samples under various multiaxial step-loading histories. The modified model was also found capable of predicting ratcheting in the opposite direction as the tensile axial mean stress dropped in magnitude. The O-W, J-S, McDowell and C-J-K models holding more backstress components and coefficients require longer Central Processing Unit (CPU) time. While time required for ratcheting assessment using the modified hardening rule was found to be twice shorter due to its simpler framework and limited number of coefficients. 


\section{ACKNOWLEDGEMENTS}

I would like to express my very great appreciation to my supervisor, Professor A. VarvaniFarahani whose guidance, expertise and encouragement were greatly helpful to me throughout my $\mathrm{PhD}$ research. He has provided me with inspiration, advice, and support, helping me to address the challenges of my dissertation. He has definitely contributed to the success of this work very much.

Sincere thanks are extended to Dr. G. R. Ahmadzadeh, Dr. M. R. Noban, adjunct Professor at University of Toronto and Professor Foroutan at Razi University who morally supported me over the period of this study.

The Financial supports through Natural Sciences and Engineering Research Council (NSERC) of Canada are greatly appreciated.

My sincerest appreciation for my father, sister and brother for all the caring and support provided to me during the time of my $\mathrm{PhD}$ studies.

Finally my warmest gratitude to my lovely wife for her patience, inspiration, unconditional support, and love. I undoubtedly owe her greatly for being able to complete my studies and perform this work. This dissertation is dedicated to her. 


\section{TABLE OF CONTENTS}

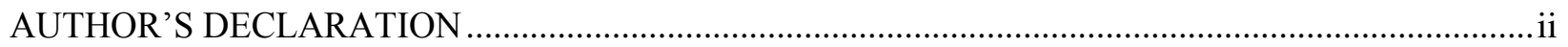

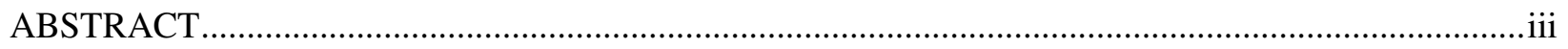

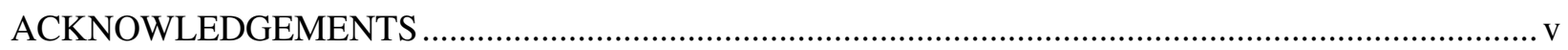

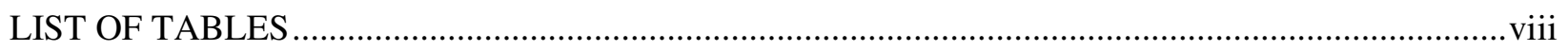

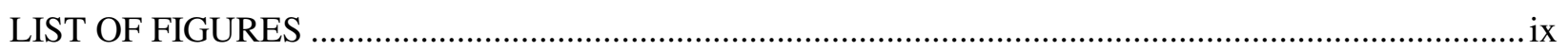

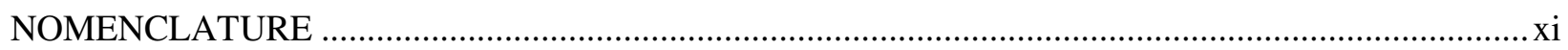

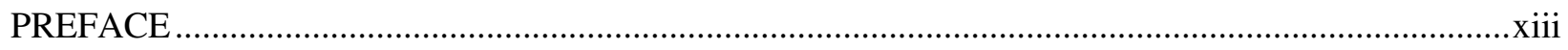

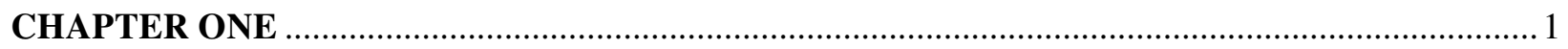

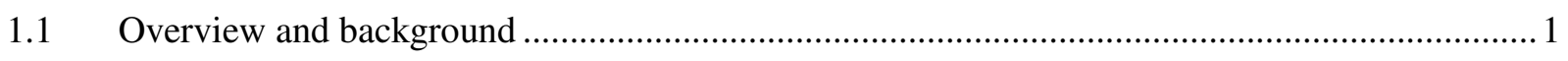

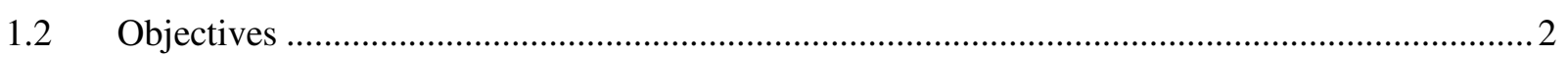

1.3 Significance of ratcheting and contribution of this research................................................. 3

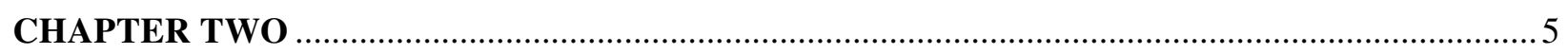

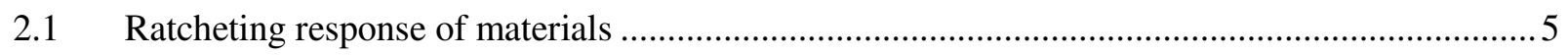

2.1.1 Coupled kinematic hardening rules and ratcheting assessment .......................................... 5

2.1.2 Hardening rules and ratcheting assessment under complex loading conditions ................... 8

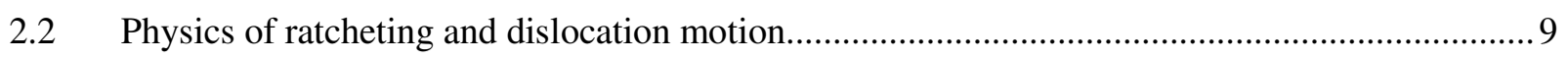

2.3 A-F type kinematic hardening rules and ratcheting assessment ........................................... 9

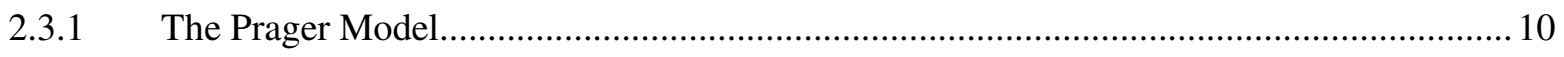

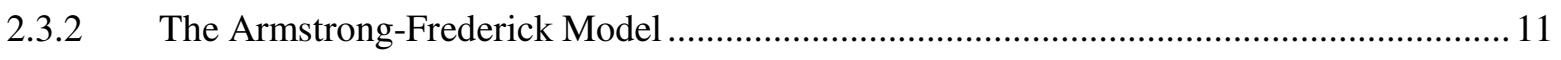

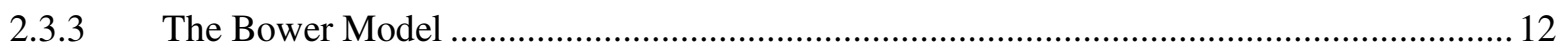

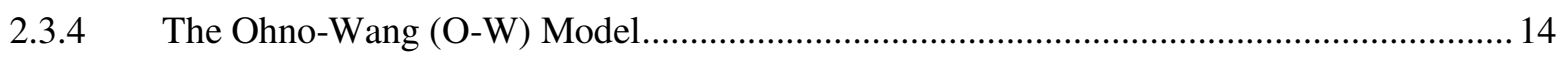

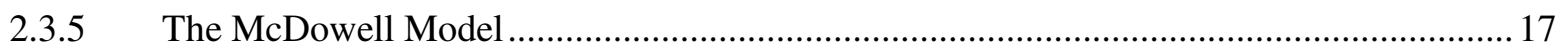

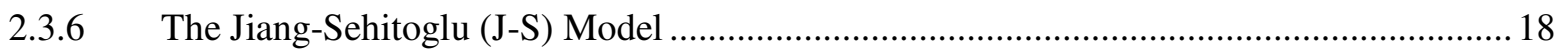

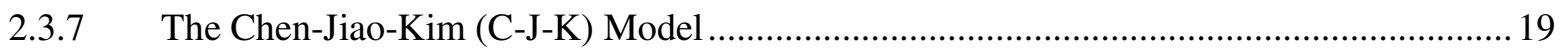

2.3.8 The Ahmadzadeh-Varvani (A-V) Model ........................................................................ 20

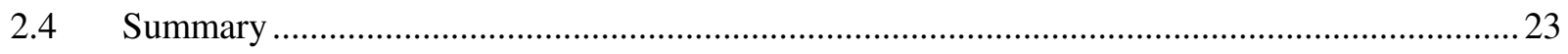

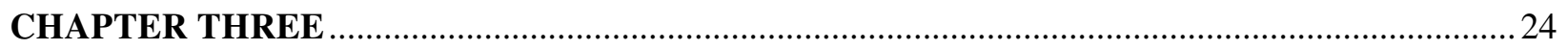

3.1 Cyclic plasticity and A-F type kinematic hardening rules .................................................24

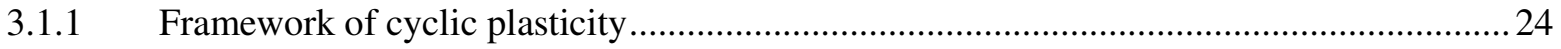




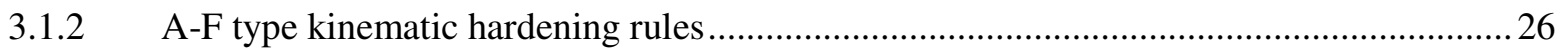

3.2 Modifications on the A-V hardening rule and dynamic recovery term .................................22

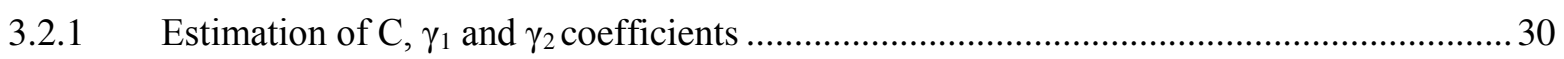

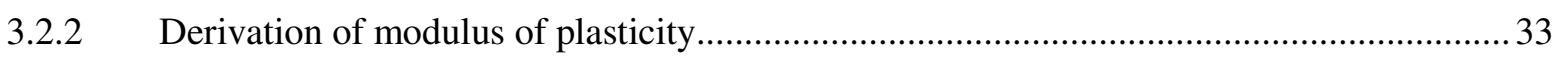

3.3 Algorithm of ratcheting assessment under various multiaxial loading conditions .....................35

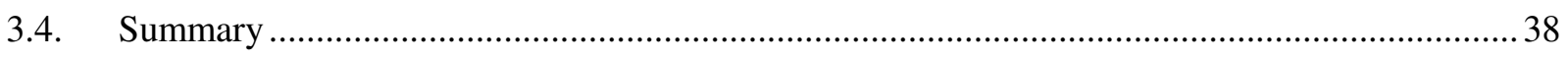

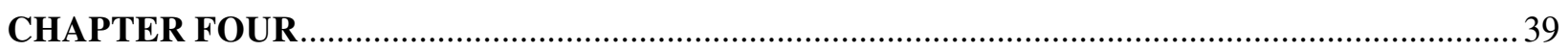

4.1 The evolution of the hardening rule in ratcheting assessment of 1045 steel alloy under

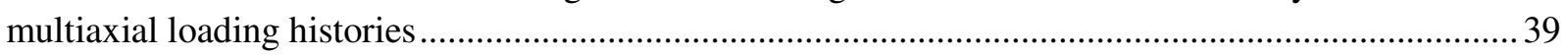

4.1.1 1045 steel samples, testing and multiaxial ratcheting data ............................................. 40

4.1.2 Predicted ratcheting curves of 1045 steel alloy............................................................ 45

4.2 Multiaxial ratcheting assessment of 1045 and 1Cr18Ni9Ti steel alloys based on the O-W, J-S,

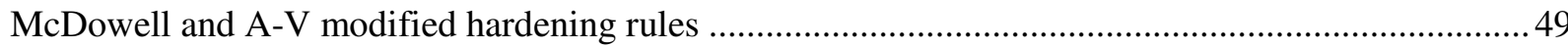

4.2.1 1045 and $1 \mathrm{Cr} 18 \mathrm{Ni}$ TTi steel samples, testing and multiaxial ratcheting data ....................50

4.2.2 Predicted ratcheting of 1045 and $1 \mathrm{Cr} 18 \mathrm{Ni}$ Ti steel alloys by O-W, J-S, McDowell and A-

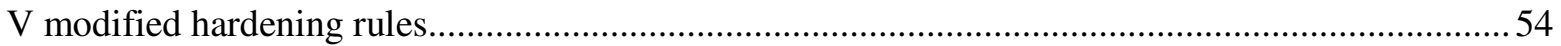

4.3 Multiaxial ratcheting of SS304 alloys under stress-controlled and combined stress- and strain-

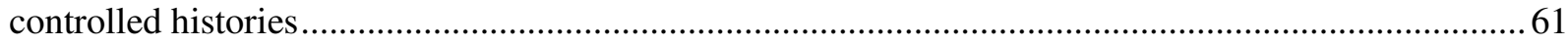

4.3.1 SS304 steel samples, testing and multiaxial ratcheting data ...........................................62

4.3.2 Predicted ratcheting of SS304 steel by O-W, C-J-K and A-V modified hardening rules

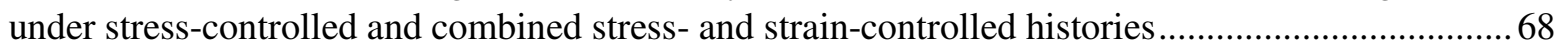

4.4 Ratcheting assessment of SS304 steel under multiaxial step-loading conditions ......................75

4.4.1 SS304 sample, testing and multiaxial ratcheting data...................................................... 75

4.4.2 Multiaxial ratcheting of SS304 over loading steps by the modified model .......................79

4.4.3 Multiaxial ratcheting of SS304 based on the O-W, C-J-K and modified A-V hardening

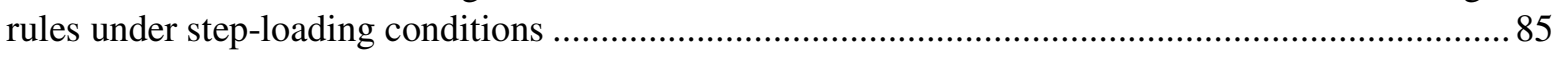

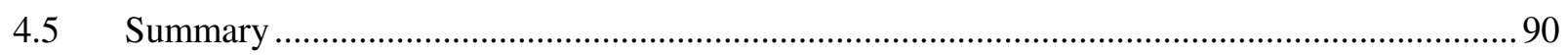

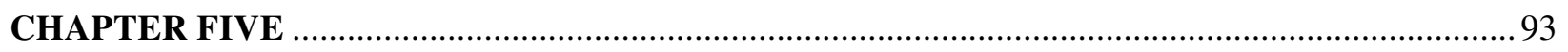

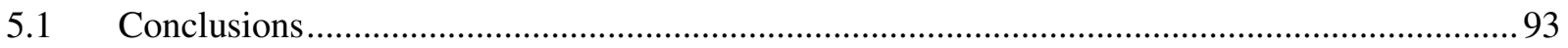

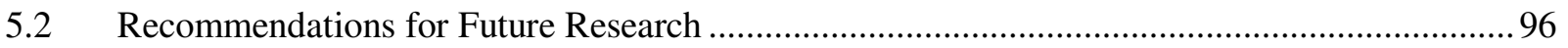

APPENDIX A

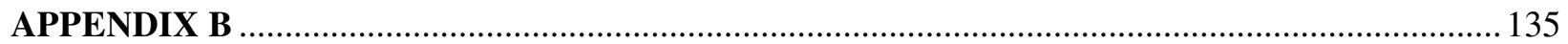

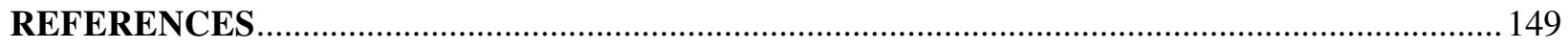




\section{LIST OF TABLES}

Table 4.1 Ratcheting experiments for 1045 steel samples under various loading paths [30]. .................. 43

Table 4.2 The coefficients of the modified hardening rule for 1045 steel alloy. .....................................45

Table 4.3 Ratcheting experiments for 1045 and 1Cr18Ni9Ti steel samples under various loading ..........52

Table 4.4 The coefficients of the hardening rules to assess ratcheting response of 1045 and $1 \mathrm{Cr} 18 \mathrm{Ni} 9 \mathrm{Ti}$

steel samples.

Table 4.5 Multiaxial ratcheting testing for 304 stainless steel samples under various loading paths.

Table 4.6 The coefficients of the hardening rules to assess ratcheting response of 304 stainless steel samples.

Table 4.7 Step-loading multiaxial ratcheting testing of SS304 samples under various loading paths [50].

Table 4.8 Coefficients of the hardening rules to assess ratcheting response of 304 stainless steel samples.

Table A.1 Symbols and terms used in the MATLAB programming codes. ........................................... 98

Table B.1 Mechanical properties of steel alloys studied in this thesis.

Table B.2 Uniaxial experimental ratcheting data of 1045 steel alloy [30] used to determine calibrating coefficient $\gamma_{2}$ in figure 4.3

Table B.3 Multiaxial ratcheting data of 1045 steel alloy [30] for various loading paths A-H used in figures 4.4 and 4.7 .

Table B.4 Multiaxial ratcheting data of 1Cr18Ni9Ti steel alloy [29] for loading paths I-K used in figure 4.8 .

Table B.5 Uniaxial experimental ratcheting data of SS304 steel alloys [47, 50-51] used to determine calibrating coefficient $\gamma_{2}$ in figure 4.12

Table B.6 Multiaxial ratcheting data for SS304 alloys [47, 50] for loading paths A1-A5 under stresscontrolled loading conditions used in figure 4.13.

Table B.7 Multiaxial ratcheting data for SS304 alloys [47, 51] for loading paths B1-B6 under mixed strain- and stress-controlled conditions used in figure 4.14.

Table B.8 Ratcheting data for SS304 alloys [43, 50] for loading paths A-E under various multiaxial steploading spectra used in figure 4.17-4-22. 


\section{LIST OF FIGURES}

Figure 2.1 Hysteresis loops based on Prager hardening rule for 42CrMo............................................ 11

Figure 2.2 Hysteresis loops of 42CrMo based on A-F hardening rule .................................................. 12

Figure 2.3 Hysteresis loops of 42CrMo based on the Bower hardening rule. ....................................... 14

Figure 2.4 Total backstress defined based on the superposition of $\mathrm{M}$ independent backstress components.

Figure 2.5 The $\mathrm{i}^{\text {th }}$ component of uniaxial backstress at different activation functions 16

Figure 2.6 The defined segments on the uniaxial cyclic stress-strain to determine the coefficients of the

$\mathrm{O}-\mathrm{W}$ hardening rule. .17

Figure 2.7 Family curves representing the variations of (a) coefficient $\gamma_{2}$ and (b) coefficient $\delta$ for different $\sigma_{\mathrm{a}}$ and $\sigma_{\mathrm{m}}$ values [34]. .22

Figure 2.8 The variation of coefficients $\delta$ and $\gamma_{2}$ on the ratcheting strain over stress cycles [34]. .23

Figure 3.1 Experimental data [30] and predicted ratcheting strain values of 1045 steel samples based on the modified model. 30

Figure 3.2 The effect of $\mathrm{C}$ and $\gamma_{1}$ on the consistency condition and hysteresis loops [35]..................... 32

Figure 3.3 Determination of coefficient $\gamma_{2}$ in the modified model using uniaxial ratcheting response..... 33

Figure 3.4 Algorithm for multiaxial ratcheting assessment based on the modified hardening rule........... 37

Figure 4.1 Multiaxial loading paths A-H and their loading spectra for 1045 steel samples. 41

Figure 4.2 Ratcheting strain vs stress cycles for 1045 steel samples under various non-proportional loading paths $(\mathrm{A}-\mathrm{H})[30]$...... 44

Figure 4.3 Determination of coefficient $\gamma_{2}$ in 1045 steel sample tested under uniaxial condition. 45

Figure 4.4 Experimental data [30] and predicted ratcheting strain values of 1045 steel samples based on the modified model under various loading conditions (A-H). 46

Figure 4.5 Multiaxial loading paths and their loading spectra for 1045 (A-H) and 1Cr18Ni9Ti (I-K) steel samples

Figure 4.6 Ratcheting strain vs stress cycles for $1 \mathrm{Cr} 18 \mathrm{Ni} 9 \mathrm{Ti}$ (I-K) steel samples under various nonproportional loading paths [29].

Figure 4.7 Experimental data [30] and predicted ratcheting strain values of 1045 steel samples based on the Ohno-Wang (O-W), Jiang-Sehitoglu (J-S), McDowell and A-V modified hardening rules under various loading conditions $(\mathrm{A}-\mathrm{H})$. 
Figure 4.8 Experimental data [29] and predicted ratcheting strain values of 1Cr18Ni9Ti steel samples based on the Ohno-Wang (O-W), Jiang-Sehitoglu (J-S), McDowell and A-V modified hardening rules

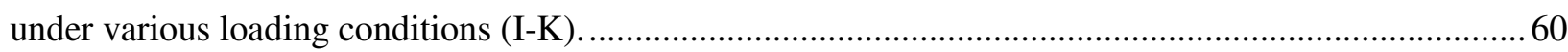

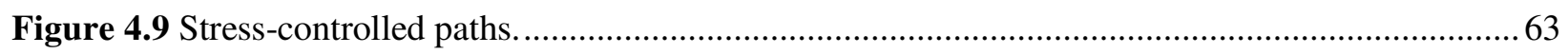

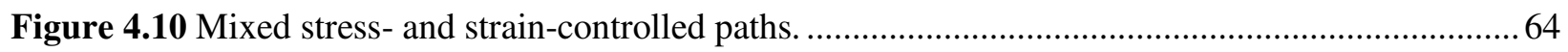

Figure 4.11 Ratcheting strain vs stress cycles for 304 stainless steel alloys under non-proportional stresscontrolled conditions (A1-A5) and mixed stress- and strain-controlled conditions (B1-B6)...................66

Figure 4.12 Determination of coefficient $\gamma_{2}$ for SS304 samples from uniaxial ratcheting data............... 67

Figure 4.13 Experimental and predicted ratcheting strain values of 304 stainless steel samples based on the $\mathrm{O}-\mathrm{W}, \mathrm{C}-\mathrm{J}-\mathrm{K}$ and A-V modified hardening rules under various stress-controlled paths A1-A5 .......... 71

Figure 4.14 Experimental and predicted ratcheting strain values of 304 stainless steel samples based on the O-W, C-J-K and A-V modified hardening rules under combined stress- and strain-controlled tests B1-

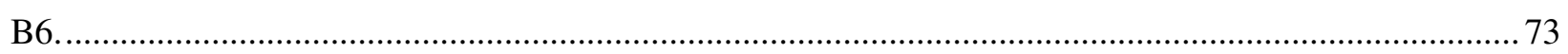

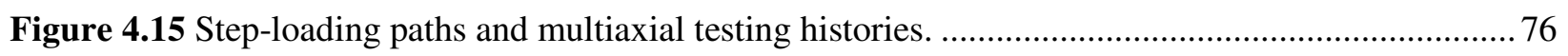

Figure 4.16 Ratcheting strain vs stress cycles for 304 stainless steel samples tested under multiaxial steploading histories A-E [50]... .78

Figure 4.17 (a) Experimental ratcheting data [50] vs predicted ratcheting curves based on the modified model, (b) experimental hysteresis loops [50] ( $\varepsilon-\gamma)$, and (c) predicted hysteresis loops $(\varepsilon-\gamma)$ over load steps for history A.

Figure 4.18 (a) Experimental ratcheting data [50] vs predicted ratcheting curves based on the modified model, (b) experimental hysteresis loops [50] $(\varepsilon-\gamma)$, and (c) predicted hysteresis loops $(\varepsilon-\gamma)$ over load steps for history B.

Figure 4.19 (a) Experimental ratcheting data [50] vs predicted ratcheting curves based on the modified model, (b) experimental hysteresis loops [43] ( $\varepsilon-\gamma)$, and (c) predicted hysteresis loops $(\varepsilon-\gamma)$ over load steps for history $\mathrm{C}$.

Figure 4.20 (a) Experimental ratcheting data [43] vs predicted ratcheting curves based on the modified model, (b) experimental hysteresis loops [50] $(\varepsilon-\gamma)$, and (c) predicted hysteresis loops $(\varepsilon-\gamma)$ over load steps for history D.

Figure 4.21 (a) Experimental ratcheting data [50] vs predicted ratcheting curves based on the modified model, (b) experimental hysteresis loops [43] ( $\varepsilon-\gamma)$, and (c) predicted hysteresis loops $(\varepsilon-\gamma)$ over load steps for history $\mathrm{E}$.

Figure 4.22 Experimental data [50] and predicted ratcheting strain values of 304 stainless steel samples based on the O-W, C-J-K and A-V modified hardening rules under various loading histories A-E. 87 


\section{NOMENCLATURE}

\begin{tabular}{|c|c|}
\hline$f$ & Yield surface function \\
\hline $\bar{S}$ & Deviatoric stress tensor \\
\hline $\bar{a}$ & Total Backstress tensor \\
\hline$\sigma_{y}$ & Yield stress \\
\hline$d \bar{\varepsilon}_{t}$ & Total strain increment \\
\hline$d \bar{\varepsilon}_{p}$ & Plastic strain increment \\
\hline$d \bar{\varepsilon}_{e}$ & Elastic strain increment \\
\hline$d \bar{\sigma}$ & Stress increment \\
\hline$G$ & Shear modulus \\
\hline$v$ & Poisson's ratio \\
\hline$E$ & Young's Modulus \\
\hline$I$ & Unit tensor \\
\hline $\bar{\sigma}$ & Stress tensor \\
\hline$H_{p}$ & Plastic modulus function \\
\hline$d \bar{s}$ & Deviatoric stress increment \\
\hline $\bar{n}$ & Unit exterior normal to the present yield surface at the stress state \\
\hline $\mathrm{C}$ & Material constant in Prager, A-F and Bower hardening rules \\
\hline$\gamma$ & Material constant in A-F hardening rule \\
\hline$d p$ & Increment of equivalent plastic strain \\
\hline$\gamma_{1}, \gamma_{2}$ & $1^{\text {st }}$ and $2^{\text {nd }}$ feedback rate of Bower hardening rule \\
\hline $\bar{b}$ & Second kinematic variable in Bower hardening rule \\
\hline$d \bar{a}_{i}$ & Increment of $\mathrm{i}^{\text {th }}$ backstress tensor \\
\hline$\gamma_{i}, r_{i}, m_{i}$ & $\begin{array}{l}\text { Material dependent constant in Ohno-Wang, McDowell, Jiang- } \\
\text { Sehitoglu and Chen-Jiao-Kim hardening rules }\end{array}$ \\
\hline$A_{i}, B_{i}$ & Additional constants in McDowell hardening rule \\
\hline$m_{0 i}$ & Additional constants in Jiang-Sehitoglu hardening rule \\
\hline$\chi_{i}$ & Additional constant in Chen-Jiao-Kim hardening rule \\
\hline
\end{tabular}




$\begin{array}{ll}\bar{a}_{i} & \mathrm{i}^{\text {th }} \text { backstress tensor } \\ f_{i} & \text { Heaviside function in Ohno-Wang hardening rule } \\ C, \gamma_{1} & \text { Material constants of A-V and the modified hardening rules } \\ \gamma_{2}, \delta & \text { Stress level dependent constants in the A-V hardening rule } \\ \gamma_{2} & \text { Calibrating coefficient in the modified hardening rule } \\ \varepsilon_{r} & \text { Ratcheting strain } \\ \sigma_{a} & \text { Stress amplitude } \\ \sigma_{m} & \text { Mean stress } \\ d \sigma_{x x} & \text { Stress increment in X-direction } \\ d \sigma_{y y} & \text { Stress increment in Y-direction } \\ d \sigma_{z z} & \text { Stress increment in Z-direction } \\ d \sigma_{x y} & \text { Shear stress increment } \\ d \varepsilon_{x x}^{t} & \text { Total strain increment in X-direction } \\ d \varepsilon_{y y}^{t} & \text { Total strain increment in Y-direction } \\ d \varepsilon_{z z}^{t} & \text { Total strain increment in Z-direction } \\ d \varepsilon_{x y}^{t} & \text { Total Shear strain increment }\end{array}$




\section{PREFACE}

A brief description of materials covered in the chapters of current thesis is provided in the following.

Chapter 1 presents the background and overview for ratcheting assessment. This chapter mainly highlights the scope and objectives of the work.

Chapter 2 presents a literature survey in the field of cyclic plasticity with emphasis on the ratcheting assessment. This chapter also discusses physics of ratcheting and dislocation motion. The kinematic hardening rules of Prager, Armstrong-Frederick, Bower, Ohno-Wang, McDowell, Jiang-Sehitoglu, Chen-Jiao-Kim and Ahmadzadeh-Varvani were reviewed in the details of their frameworks.

Chapter 3 discusses the framework, terms and components of the modified hardening rule to assess multiaxial ratcheting of materials. The terms and coefficients in the dynamic recovery of the modified hardening rule are introduced in details and their contributions in multiaxial ratcheting assessment of materials in different directions, loading path and non-proportionality effect, and shakedown and ratcheting arrest were discussed. The procedures for calculation of coupled modulus of plasticity and other stress/strain components for both stress-controlled conditions and mixed stress- and strain-controlled multiaxial conditions are presented. This chapter presents a comprehensive algorithm to assess ratcheting based on the modified hardening rule for various multiaxial step-loading spectra.

Chapter 4 presents results and discussion on ratcheting assessment of steel alloys under various multiaxial loading spectra by mean of the modified hardening rule and other well-known hardening rules of $\mathrm{O}-\mathrm{W}, \mathrm{McDowell}, \mathrm{J}-\mathrm{S}$, and $\mathrm{C}-\mathrm{J}-\mathrm{K}$. The terms and coefficients of dynamic recovery in these models are extensively discussed and the capability of hardening rules in assessing ratcheting under various multiaxial step-loading conditions and paths were evaluated as the predicted ratcheting curves were compared with those experimentally reported in this chapter. 
Chapter 5 summarizes the conclusions extracted from this research work and presents future recommendations.

Appendix A presents MATLAB programming code developed to assess ratcheting response based on the modified hardening rule and other well-known hardening rules of O-W, McDowell, J-S, and C-J-K along with related subroutines.

Appendix B tabulates ratcheting experimental strain values employed in chapter 4 to evaluate the capability of the modified hardening rule and the O-W, J-S, and C-J-K models for steel alloys under various multiaxial loading paths and spectra. 


\section{CHAPTER ONE}

\section{INTRODUCTION}

\subsection{Overview and background}

Engineering components and machinery parts such as those in auto-, aero-, and pipeline industries can experience catastrophic failure if the extent of materials plastic deformation is not accurately controlled over stress cycles. Plasticity theories enable characterising nonlinear strainstress response of materials under cyclic loading conditions. Structural components experiencing the low-cycle fatigue (LCF) in the presence of mean stress can accumulate an irreversible plastic deformation in progress of their fatigue damage as the number of cycle advances. This successive and directional plastic strain is defined as cyclic ratcheting strain and occurs in components undergoing unsymmetrical stress cycles. The integration of ratcheting and fatigue phenomena results in severe damage in materials leading to failure of components. A precise investigation of ratcheting response of materials is crucial for reliable design of engineering components and structures undergoing asymmetric stress cycles.

The simulation of ratcheting strain in the framework of coupled constitutive theories is complicated as plastic deformation involves the translation of stress space as well as change in shape and size of yield surfaces. Several exciting theories in literature are yet to properly predict ratcheting behavior of materials particularly under multiaxial loading conditions. The developed models in the literature possess numerous coefficients and complicated frameworks. These models are also limited to address ratcheting response of some particular materials and limited experimental ratcheting data. 
The present study intends to assess the ratcheting response of steel alloys under single and multi-step multiaxial loading histories by means of a modified hardening rule developed on the basis of the Ahmadzadeh-Varvani (A-V) hardening rule. The capability of the modified model then will be compared with predicted ratcheting curves of earlier well-known models of OhnoWang (O-W), McDowell, Jiang-Sehitoglu (J-S) and Chen-Jiao-Kim (C-J-K) hardening rules as well as experimentally obtained ratcheting data.

\subsection{Objectives}

The present research intends to study the cyclic ratcheting behavior of different steel alloys under non-proportional loading conditions to further develop a simple framework of hardening rule for the prediction of ratcheting strain under stress-controlled and mixed stress- straincontrolled multiaxial paths. The main objective of this work is to further develop the A-F based hardening rule of $\mathrm{A}-\mathrm{V}$ for ratcheting assessment of materials under multiaxial loading histories through a comprehensive algorithm and Matlab programming code. The modified hardening rule holding material dependent coefficients $\mathrm{C}$ and $\gamma_{1}$ of the $\mathrm{A}-\mathrm{V}$ model, redefined calibrating coefficient $\gamma_{2}$ in conjunction with terms of $\left\langle d \bar{\varepsilon}_{p} \cdot \bar{a} /|\bar{a}|\right\rangle,\langle\bar{n} \cdot \bar{a} /|\bar{a}|\rangle^{1 / 2}$ and $(2-\bar{n} \cdot \bar{a} /|\bar{a}|)$ in the dynamic recovery of the model. The projection presented by the function in term $\left\langle d \bar{\varepsilon}_{p} \cdot \bar{a} /|\bar{a}|\right\rangle$ is smaller than $d p$ in the $\mathrm{A}-\mathrm{V}$ model resulting in a slower evolution in simulation of ratcheting strain under multiaxial loading conditions. Term $\left\langle d \bar{\varepsilon}_{p} \cdot \bar{a} /|\bar{a}|\right\rangle$ introduces a slower evolution of ratcheting strain in the deviatoric stress space as the components $d \bar{\varepsilon}_{p}$ and $\bar{a} /|\bar{a}|$ in the MaCaulay brackets take different directions under multiaxial loading conditions and makes the model capable of tracking these different directions. The measure of non-proportionality was also utilized in the form of product of $\langle\bar{n} \cdot \bar{a} /|\bar{a}|\rangle^{1 / 2}$ and vector $\bar{b}$ to control the accumulation rate of ratcheting strain and mainly to prevent the modified model to experience plastic shakedown as the number of cycles advances over various multiaxial loading conditions. Under multiaxial loading histories, term 
$\langle\bar{n} \cdot \bar{a} /|\bar{a}|\rangle^{1 / 2}$ takes values smaller than 1 and prevents this model from premature plastic shakedown. Term $(2-\bar{n} \cdot \bar{a} /|\bar{a}|)$ regulates the magnitude of coefficient $\gamma_{2}$ properly for various multiaxial loading paths. This term takes the values between 1 and 3 under multiaxial loading and consequently magnifies the effect of $\gamma_{2}$ and results in a smaller accumulation rate of ratcheting strain over stress cycles. Term $(2-\bar{n} \cdot \bar{a} /|\bar{a}|)$ encounters the effect of non-proportionality as multiaxial loads are applied and reduces to unity for uniaxial loading condition.

The capability of the modified hardening rule in ratcheting assessment of materials is further examined and compared with a wide variety experimentally obtained data under singlestep and multi-step non-proportional loading histories. The framework, terms and coefficients of the Ohno-Wang (O-W), McDowell, Jiang-Sehitoglu (J-S) and Chen-Jiao-Kim (C-J-K) hardening rules along with the modified $\mathrm{A}-\mathrm{V}$ model are described in details. The capability, limitations and complications involved with multiaxial ratcheting assessment of steel alloys are discussed.

\subsection{Significance of ratcheting and contribution of this research}

Structural and engineering components subjected to asymmetric stress cycles are prone to failure and require to be critically assessed for materials integrity. Excess of applied stress in load-bearing components in service beyond the elastic limit is most times inevitable. Successive and directional plastic strain accumulation over stress cycles and its integration with fatigue damage deteriorates fatigue damage through a continuous drop in cross-sectional area of components. The ratcheting strain is a hidden problem and exacerbates performance of engineering structures due to the interaction of fatigue damage and accumulated ratcheting strain. This phenomenon is influenced by several parameters including microstructural properties, stress amplitude, mean stress, loading history, loading step and the sequence of loading. The catastrophic failure of engineering components and structures has been reported [1] to cost over 120 billion dollars per year in North America and ratcheting phenomenon has 
been recognised as one of the main root causes due to its high detrimental influence on the components life. Research in ratcheting enhances a better understanding of the mechanism involved with plastic strain accumulation over stress cycles and offers pertinent database to reliably design components such as landing gears, offshore structures and piping /pressure vessels undergoing asymmetric stresses.

Ratcheting assessment is considered as a challenging engineering problem due to complexity in constitutive cyclic plasticity equations. This involves a delegated process in yield surface translation over loading within plasticity regime through a proper choice of kinematic hardening rule. This research contributes to develop a comprehensive kinematic hardening rule to assess ratcheting response of steel alloys and addresses shortcomings of earlier developed hardening rules. The following outlines contributions of the current study:

* The modified hardening rule offers a simple framework with less coefficients to assess ratcheting response of materials over various multiaxial loading spectra.

* Terms introduced in the dynamic recovery holding plastic strain increment, the normal vector to the yield surface, and the backstress components enable the modified model to (i) track different directions, (ii) account for the effect of non-proportionality, and (iii) prevent plastic shakedown over the multiaxial stress cycles.

* Coefficients in the modified hardening rule are defined from uniaxial stress-strain and ratcheting data while constants in other models discussed in this research are defined by segments chosen on the uniaxial stress-strain and multiaxial ratcheting curves through a tedious and time-consuming trial procedure.

- The modified model with less complexity and number of coefficients noticeably shortens the CPU time in ratcheting assessment as compared to other hardening rules discussed in this thesis. 


\section{CHAPTER TWO}

\section{LITERATURE SURVEY}

\subsection{Ratcheting response of materials}

Structural components and machinery parts undergo failure if materials deform severely over stress cycles. Engineering components experiencing asymmetric stress cycles with non-zero mean stress accumulate an irreversible plastic deformation along with fatigue damage as the number of cycles progresses. This successive and directional accumulation of plastic strain is referred as ratcheting strain. Both ratcheting and fatigue phenomena when are coupled result in a severe damage leading to failure of components. For reliable design of engineering components and structures undergoing asymmetric stress cycles, the ratcheting assessment of materials is always crucial. Ratcheting phenomenon was first reported by Bairstow [2]. This subject has received considerable attention over last few decades. Many researchers have investigated cyclic plasticity and ratcheting response of various materials tested under stress-controlled conditions [3$12]$.

\subsubsection{Coupled kinematic hardening rules and ratcheting assessment}

Several cyclic plasticity models have been developed to characterize ratcheting response of materials under various loading conditions. Unlike coupled hardening rules, there are some uncoupled models structured on the basis of constitutive equations at which the calculation of the modulus of plasticity, $H_{p}$, is not coupled with the hardening rule through the consistency condition of yield surface. It can however indirectly affect the hardening rule. The current study aims to study and evaluate the coupled constitutive models in predicting and modeling of cyclic ratcheting 
strain of materials. The coupled kinematic hardening rules at which the calculation of the modulus of plasticity, $H_{p}$, was coupled with the hardening rule through the consistency condition of yield surface were proposed by Armstrong-Frederick (A-F) [13], Chaboche [14], Bower [15], Bari and Hassan [16], Delobelle et al. [17], Burlet-Cailletaud [18], Ohno-Wang (O-W) [19-20], JiangSehitoglu (J-S) [21, 22], McDowell [23] , Abdel Karim-Ohno [24, 25], Döring et al. [26], Kang [27], Chen et al. [28-30], Yaguchi-Takahashi [31], Colak [32], Dafalias-Feigenbaum [33] and lately Ahmadzadeh-Varvani (A-V) [34, 35]. Combination of linear strain hardening and dynamic recovery terms have constructed coupled nonlinear constitutive model of A-F. The choice and capability of the coupled hardening rules has been highly dependent on how dynamic recovery term was modified. To decrease the rate of plastic strain accumulation over stress cycles, Bower [15] introduced a simple non-linear model by adding the second kinematic variable. Chaboche [14] proposed a model with a threshold in the dynamic recovery term. Chaboche [14] extended AF hardening rule by decomposing hardening rule to several parts at which backstress components worked independently. The main goal of employing a threshold in the hardening rule was to reduce the overall magnitude of ratcheting and it was implemented by means of a term inside MaCaulay brackets. During plastic deformation, the threshold converted the model from non-linear to linear hardening rule. Beyond this threshold, the recall term prevented plastic shakedown to occur. Bari and Hassan [36] achieved better simulation of uniaxial ratcheting strain while the model with threshold over-predicted ratcheting strain for multiaxial loading conditions. Bari and Hassan [16] developed a hardening rule by using the ideas of Delobelle et al. [17]. Depending on the magnitude of Delobelle parameter, this model compromises over-prediction of ratcheting of A-F and the plastic shakedown of Burlet-Cailletaud [18] under multiaxial loading conditions. Dafalias et al. $[37,38]$ developed multiplicative A-F kinematic hardening rule based on the initial model of Chaboche et al. [39].

Based on the concept of independent backstress components Ohno and Wang [19] developed a multilinear hardening rule in which each backstress component would have a critical state. To avoid producing closed hysteresis loops in uniaxial loading condition, the second version 
of O-W model was introduced [19] through a power-law function. To improve the capability of this model in predicting ratcheting strain under various loading conditions, the $\mathrm{O}-\mathrm{W}$ models have been taken as the backbone of several hardening rules through modifications on dynamic recovery part. Abdel-Karim [40] examined the recently developed models implementing terms/ variables introduced into the dynamic recovery term of O-W. Jiang and Sehitoglu [22] and McDowell [23] modified the exponent of the O-W model. However in these models, accumulated plastic strain increment was employed rather than plastic strain increment incorporated to the dynamic recovery term. To improve the ability of first model of $\mathrm{O}-\mathrm{W}$ rather than non-linear activation, Abdel-Karim and Ohno [24] utilized a weighting parameter to partially activate dynamic recovery term as backstress component reached its critical value. Another effort to improve ratcheting simulation was made by Abdel-Karim and Ohno [25] by multiplying new parameters as additional terms into dynamic recovery of $\mathrm{O}-\mathrm{W}$ initial model. Magnitude of these parameters are able to compromise the over-prediction of A-F and shakedown of O-W under uniaxial loading conditions. Chen et al. [30] reported that the magnitudes of these parameters determined based on uniaxial ratcheting test did not enable this hardening rule to predict multiaxial ratcheting in materials. Kang [27] has utilized accumulated plastic strain increment instead of plastic strain increment in this model to develop a visco-plastic constitutive model to simulate uniaxial and multiaxial ratcheting responses of U71Mn rail steel samples. Chen et al. [30] developed a model incorporating a new factor associated with backstress and non-coaxiality of plastic strain rate in the second model of O-W. Hardening rule of Chen et al. [30] in simulating materials ratcheting under multiaxial loading conditions were reported promising [40]. Ahmadzadeh-Varvani [34, 35] modified the dynamic recovery term in Bower's kinematic hardening rule by means of a limited number of material and stress level dependent coefficients to assess uniaxial ratcheting response of materials. 


\subsubsection{Hardening rules and ratcheting assessment under complex loading conditions}

Multiaxial ratcheting response of materials becomes rather challenging as loading path and non-proportionality are coupled with the hardening rules. Non-proportional loading histories induce greater hardening than those of proportional, resulting in slower rates in the ratcheting progress over multiaxial stress cycles [41]. The ratcheting assessment of materials are investigated based on stress-controlled and/or combined stress- and strain-controlled multiaxial testing conditions. Under stress-controlled state, the elements of applied stress tensor are known and the incremental ratcheting strain values are defined using Hooke's law and flow rule through the framework of the kinematic hardening rule. While the combined condition embraces complexity in the tensorial stress and strain components before the deviatoric stress/strain components are employed in ratcheting analysis of materials. Several researchers [41-52] have discussed these complexities including the effect of complex loading paths and non-proportionality on ratcheting response of materials conducting multiaxial ratcheting tests under stress-controlled and the combined stress- and strain-controlled conditions. Hassan et al. [5, 8, 47] discussed capability of hardening rules in ratcheting assessment of materials under multiaxial loading conditions and concluded that the non-proportionality effect is yet to fully address and the shape of hysteresis curves and the evolution of yield surfaces over stress cycles are required to be accurately predicted.

Jiang and Sehitoglu [53] examined ratcheting response of 1070 steel samples under uniaxial and multiaxial step-loading histories. They investigated ratcheting response of steel samples under uniaxial high-low and low-high step-loading histories as well as two-step axialtorsional loading paths. Haupt and Schinke [54] conducted two-step loading tests on austenitic AISI 316 L (N) stainless steel samples at room temperature and discussed the influence of loading sequence on ratcheting response of steel samples. The influence of loading sequence, stress amplitude and mean stress on ratcheting response of SA333 C-Mn and 304LN steel alloys over steps of loading was experimentally examined by Paul et al. [55, 56]. Kang et al. [27, 43, 50, 5759] investigated ratcheting response of 304 and 316 stainless steel alloys under uniaxial and multiaxial step-loading histories. In their studies, Kang and coworkers [27] discussed the influence 
of temperature in ratcheting progress over loading steps. Goodman [60] investigated the ratcheting response of SS316 steel samples under multi-step loading histories and discussed how influential the effect of increase in mean stress on ratcheting magnitude is while the applied stress amplitude is kept constant. On the other hand, Hassan and Kyriakides [4] discussed the ratcheting response of 1026 steel samples tested under multi-step loading with an increasing stress amplitude and a constant mean stress over loading steps. Ahmadzadeh-Varvani [35] evaluated ratcheting response of SS316L, SA333, SS316L (N) and 1070 steel alloys undergoing various uniaxial low-high, highlow, low-high-low and high-low-high loading sequences by means of the A-V hardening rule and discussed the influence of the prior load step for different loading sequences affecting the ratcheting progress in subsequent steps.

\subsection{Physics of ratcheting and dislocation motion}

The successive plastic deformation over stress cycles referred as ratcheting strain tends to be accumulated in three distinct stages. This phenomenon is similar, but not identical in nature to cyclic creep. The ratcheting strain is the result of formation of cells, dislocations movement and/or slip and the interaction of dislocations [61-64]. In stage I, ratcheting increases rapidly while experiencing a gradual decrease in ratcheting rate as a result of cyclic hardening and reduction of dislocation motion [65]. In the second stage, the ratcheting rate decreases until the stabilization of ratcheting strain occurs. At the last stage, as the number of cycles advances more active dislocation are produced and the cross sectional area decreases due to accumulation of the plastic strain. This accelerates the accumulation of ratcheting strain, resulting in an increase in the true stress, and an instability and therefore necking in the specimen experiencing ratcheting [61-64].

\subsection{A-F type kinematic hardening rules and ratcheting assessment}

The backbone of cyclic plasticity models is structured by important constituents of strain increment, Hooke's law, yield function, flow rule, hardening rule and consistency condition. The 
hardening rule is the central part of cyclic plasticity theory defining the movement direction of yield surface in the stress space during plastic deformation. This chapter mainly describes structure of A-F type hardening rules including the linear kinematic hardening rule of Prager [66], nonlinear hardening rule of Armstrong-Frederick (A-F) [13], Bower's model [15] further developed on the basis of A-F rule by introducing second kinematic variable, the Ohno-Wang (O-W) [20] hardening rule developed based on the critical state of dynamic recovery of backstress, the JiangSehitoglu (J-S) [21], McDowell and Chen-Jiao-Kim (C-J-K) [30] hardening rules developed based on the $\mathrm{O}-\mathrm{W}$ rule to include the effects of non-proportionality in ratcheting assessment of materials.

\subsubsection{The Prager Model}

The simplest kinematic hardening rule has been proposed by Prager [66]. This model translates the yield surface linearly in the stress space without altering the shape and size of it during plastic deformation:

$$
d \bar{a}=C d \bar{\varepsilon}_{p}
$$

where $\bar{a}$ corresponds to the backstress tensor and $\mathrm{C}$ is a material constant. Based on the linear hardening rule, if the shape of yield surface stays unchanged the value of plastic modulus will be identical with the value of constant $\mathrm{C}$ in forward loading and reverse loading and consequently results in closed hysteresis loops and no ratcheting as shown in Fig. 2.1. Upon application of stress cycles in the presence of mean stress, this model is not capable to predict the shape of hysteresis loops properly and after overprediction of ratcheting over initial stress cycles ratcheting is arrested. 


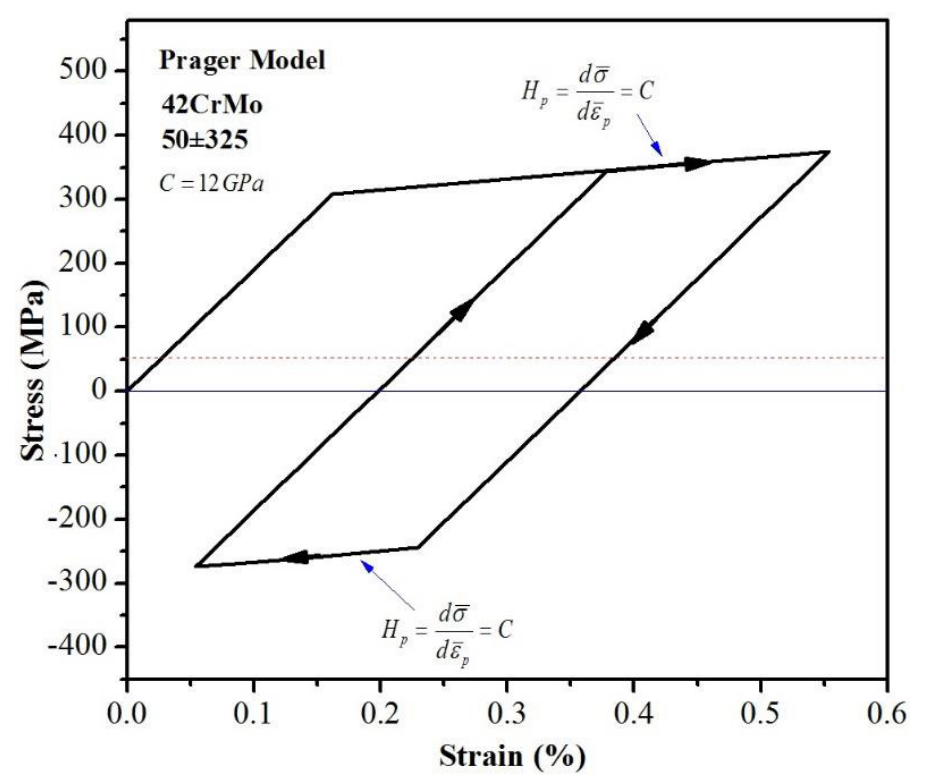

Figure 2.1 Hysteresis loops based on Prager hardening rule for 42CrMo.

\subsubsection{The Armstrong-Frederick Model}

Armstrong and Frederick [13] modified Prager's model by adding a dynamic recovery term introducing nonlinearity to this model as:

$$
d \bar{a}=C d \bar{\varepsilon}_{p}-\gamma \bar{a} d p
$$

where

$$
d p=\sqrt{d \bar{\varepsilon}_{p} \cdot d \bar{\varepsilon}_{p}}
$$

Coefficients $\mathrm{C}$ and $\gamma$ are material coefficients. The second term in equation (2.2) introducing nonlinearity to the model is responsible for the accumulation of plastic strain over the stress cycles. Uniaxial stress-strain hysteresis loops obtained from strain-controlled loading condition are employed to determine the $\mathrm{C}$ and $\gamma$. Constants $\mathrm{C}$ and $\gamma$ are used to find modulus of plasticity given in equation (2.4):

$$
H_{p}=C \pm \gamma(\bar{a} \cdot \bar{n})
$$


The dynamic recovery term in Armstrong-Frederick kinematic hardening rule is responsible to track different shapes of forward loading and reverse loading hysteresis curves. The modulus of plasticity takes negative sign during forward loading and positive sign in reverse loading as shown in the Fig 2.2 for $42 \mathrm{CrMo}$ steel.

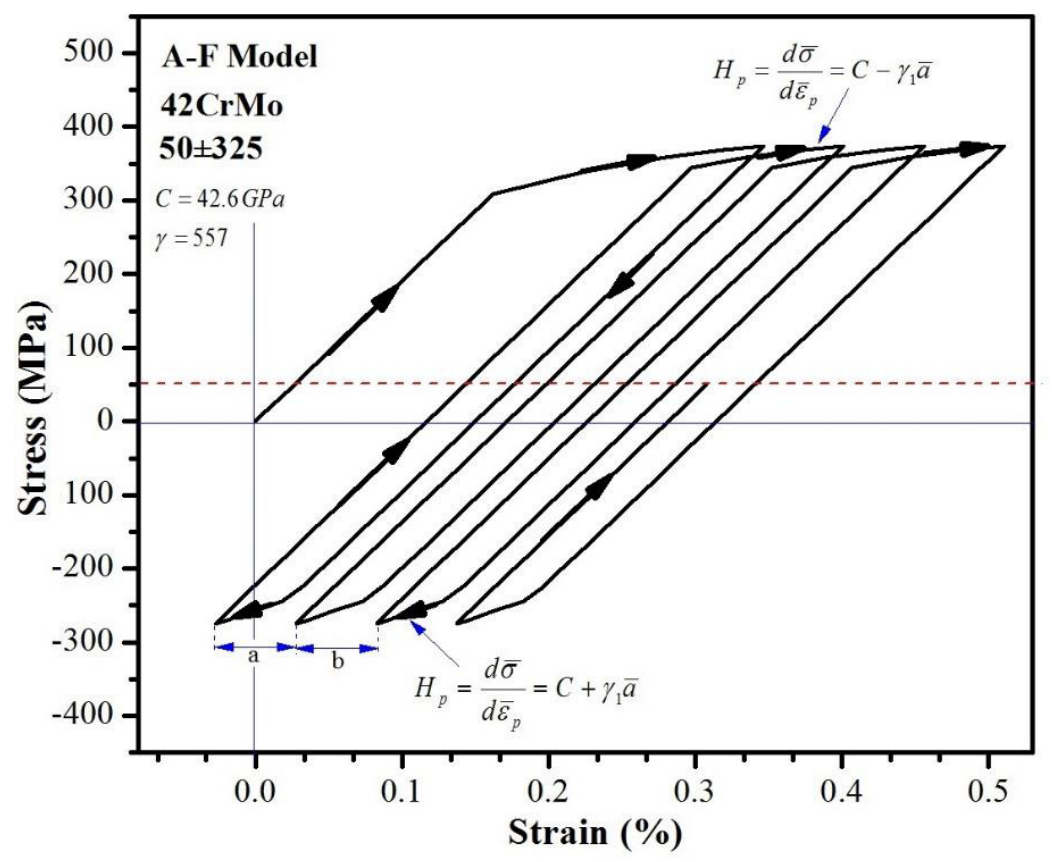

Figure 2.2 Hysteresis loops of 42CrMo based on A-F hardening rule.

The A-F hardening model produces open hysteresis loops resulting in ratcheting progress, however this model predicts same loops over all stress cycles, resulting in a constant rate of ratcheting. In Fig 2.2 the equality of ratcheting strain intervals $(a=b)$ proves that this model predicts ratcheting strain of $42 \mathrm{CrMo}$ steel with a constant rate resulting in a significant overestimation of the ratcheting.

\subsubsection{The Bower Model}

The A-F hardening rule predicts ratcheting with a constant rate per cycle and consequently overestimates the ratcheting strain. Bower [15] further modified the A-F rule to overcome this drawback. He introduced the second kinematic variable $\gamma_{2}$ into the dynamic recovery term to 
reduce the constant rate of strain accumulation predicted by the A-F hardening rule as the number of stress cycles advances. The Bower's model of equation (2.5) employs the second kinematic variable in the recall term to simulate the accumulating ratcheting strain decay $[15,67]$ :

$$
\begin{aligned}
& d \bar{a}=C d \bar{\varepsilon}_{p}-\gamma_{1}(\bar{a}-\bar{b}) d p \\
& d \bar{b}=\gamma_{2}(\bar{a}-\bar{b}) d p
\end{aligned}
$$

In Bower's model coefficient $C$ and the first kinematic variable $\gamma_{1}$ are material constants regulating size and the shape of hysteresis loops. The second kinematic variable is responsible to determine the ratcheting rate. Constant $\gamma_{2}$ introduces a gradual decrease in the magnitude of $(\bar{a}-\bar{b})$ as the stress cycles advances. The movement of tensor $\bar{b}$ with the initial value of zero in equation (2.5a) follows backstress $(\bar{a})$ exponentially through equation $(2.5 \mathrm{~b})$ over stress cycles. This model is capable to simulate rate of ratcheting decay over initial number of cycles and over stage I. Fig 2.3 shows the hysteresis loops of $42 \mathrm{CrMo}$ steel sample predicted by the Bower model. This figure shows the capability of the model to track different shapes between forward and reverse loading and produces open hysteresis loops similar to the A-F hardening rule. It is evident from figure $2.3 \mathrm{as} \mathrm{a}>\mathrm{b}$, this model is able to simulate the rate of ratcheting decay as the number of cycles advances. The model however suffers a premature shakedown in stage II. 


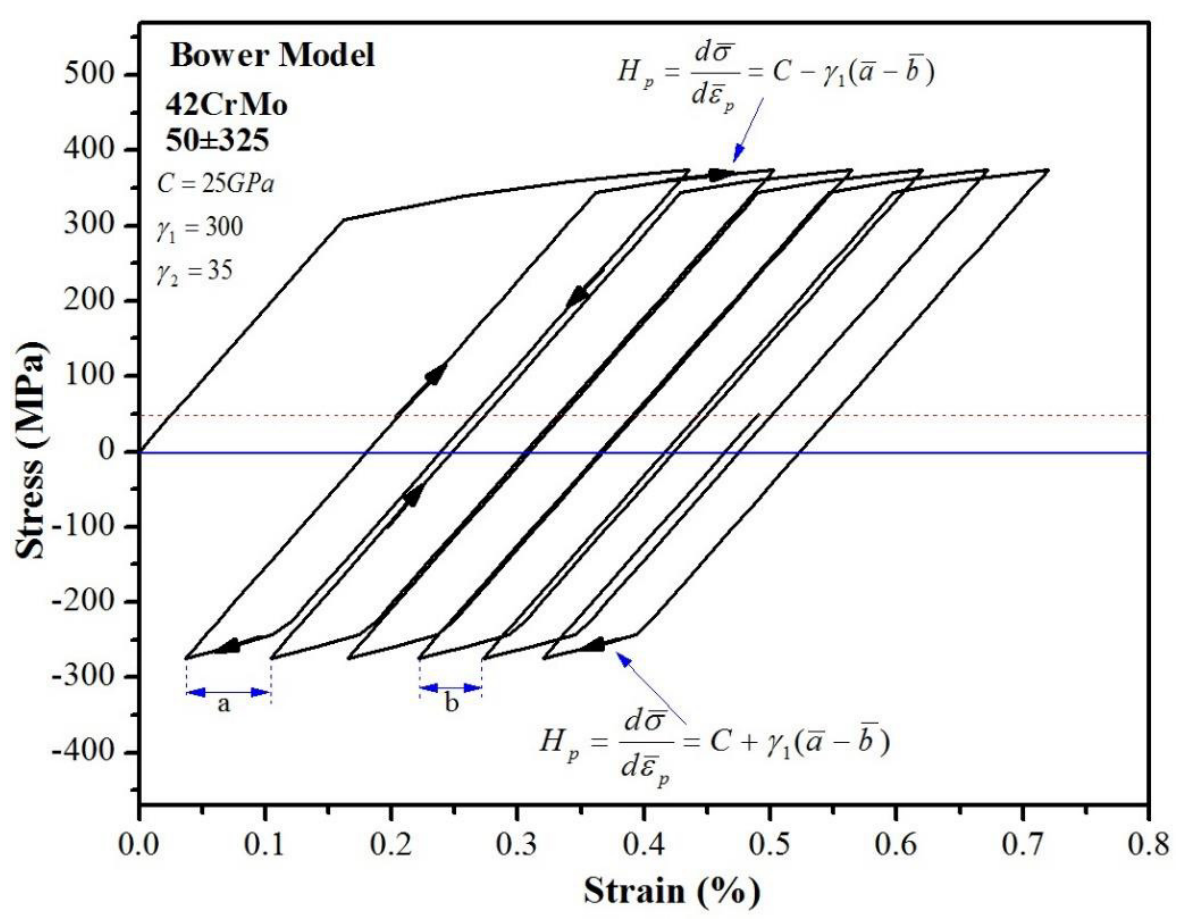

Figure 2.3 Hysteresis loops of 42CrMo based on the Bower hardening rule.

\subsubsection{The Ohno-Wang (O-W) Model}

Ohno and Wang $[19,20]$ developed a hardening rule on the basis of the critical state of the dynamic recovery term in the backstress equation. The total backstress in this hardening rule is defined based on the superposition of $M$ independent backstress components as presented schematically in figure 2.4 suggested first by Chaboche [14] as:

$$
d \bar{a}=\sum_{i=1}^{M} d \bar{a}_{i} \quad(i=1,2, \ldots, M)
$$




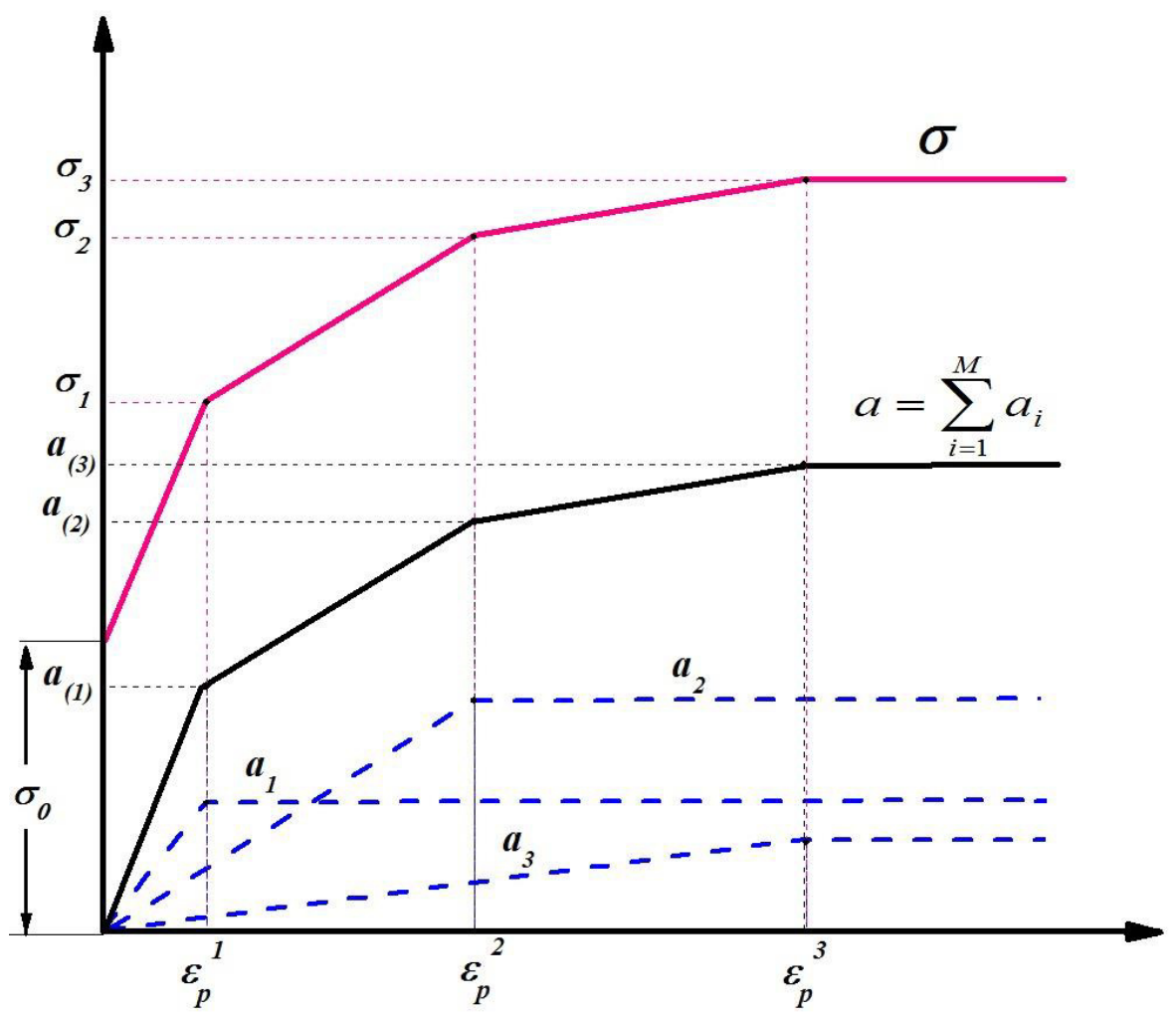

Figure 2.4 Total backstress defined based on the superposition of $M$ independent backstress components.

A critical value in each component $(i=1,2, \ldots, M)$ causes its dynamic recovery term to be fully activated. In the initial O-W model [19], authors employed a Heaviside step function in the dynamic recovery term as:

$$
d \bar{a}_{i}=\gamma_{i}\left[\frac{2}{3} r_{i} d \bar{\varepsilon}_{p}-H\left(f_{i}\right)\left\langle d \bar{\varepsilon}_{p} \cdot \frac{\bar{a}_{i}}{\left|\bar{a}_{i}\right|}\right\rangle \bar{a}_{i}\right]
$$

Magnitude of $\bar{a}_{i}$ is defined as $\left|\bar{a}_{i}\right|=\left(\bar{a}_{i} \cdot \bar{a}_{i}\right)^{1 / 2}$ and the critical state of dynamic recovery through yield surface definition of $f_{i}=\left|\bar{a}_{i}\right|^{2}-r_{i}^{2}$. In proportional loading conditions, for $\bar{a}_{i}<r_{i}$ hardening rule linearly develops with the rate of $\frac{2}{3} \gamma_{i} r_{i}$ and as soon as $\bar{a}_{i}=r_{i}$ dynamic recovery term is activated and dynamic recovery and hardening terms become balanced resulting in no change in backstress i.e. $d \bar{a}_{i}=0$. The hardening rule acted as a multi-linear model for uniaxial 
case and produced closed hysteresis loops resulting in materials ratcheting arrest $[19,68]$. To overcome this shortcoming, the second $\mathrm{O}-\mathrm{W}$ model was defined by means of a power function as:

$$
d \bar{a}_{i}=\gamma_{i}\left[\frac{2}{3} r_{i} d \bar{\varepsilon}_{p}-\left(\frac{\left|\bar{a}_{i}\right|}{r_{i}}\right)^{m_{i}}\left\langle d \bar{\varepsilon}_{p} \cdot \frac{\bar{a}_{i}}{\left|\bar{a}_{i}\right|}\right\rangle \bar{a}_{i}\right]
$$

Dynamic recovery term in this hardening rule operates partially within the yield surface $f_{i}$ and as each backstress component, $\bar{a}_{i}$ approaches the surface $f_{i}$ its dynamic recovery becomes highly nonlinear. As exponent $m_{i}$ approaches infinity, Eq. (2.8) turns to the initial O-W model and acts like a multilinear hardening rule resulting in the plastic shakedown after a slight overprediction in ratcheting [19]. Fig 2.5 shows that the variation of backstress $\alpha_{i}$ at the transient stage is affected by to the Heaviside and power functions under monotonic loading.

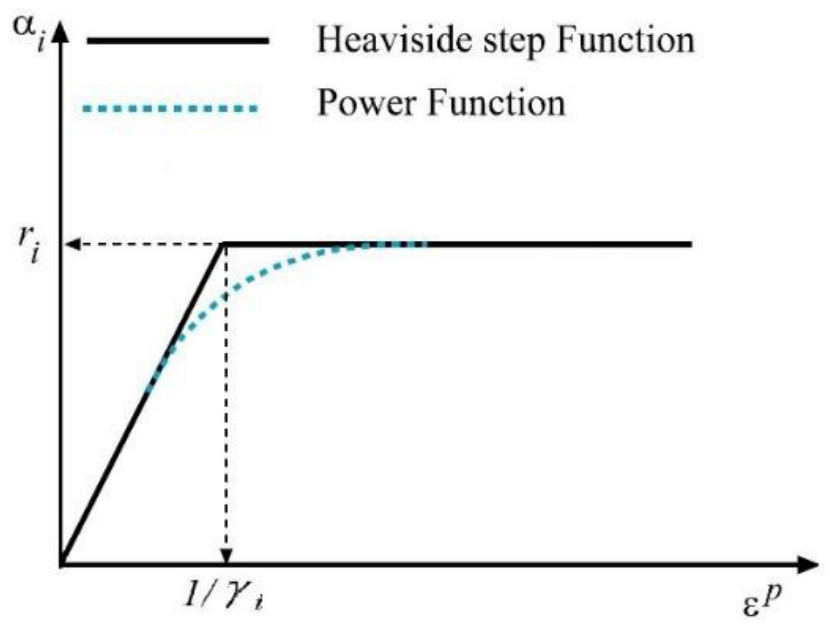

Figure 2.5 The $\mathrm{i}^{\text {th }}$ component of uniaxial backstress at different activation functions

Figure 2.6 shows how a tensile uniaxial stress-strain curve is divided into several segments, and consequently, parameters $r_{i}$ and $\gamma_{i}$ are determined through equations (2.9a) and (2.9b), respectively as:

$$
\gamma_{i}=\frac{1}{\varepsilon_{p(i)}}, \quad r_{i}=\left(\frac{\sigma_{(i)}-\sigma_{(i-1)}}{\varepsilon_{p(i)}-\varepsilon_{p(i-1)}}-\frac{\sigma_{(i+1)}-\sigma_{(i)}}{\varepsilon_{p(i+1)}-\varepsilon_{p(i)}}\right) \varepsilon_{p(i)}, \text { for } i \neq 1
$$




$$
\sum_{i=1}^{M} r_{i}+\sigma_{0}=\sigma_{\max }
$$

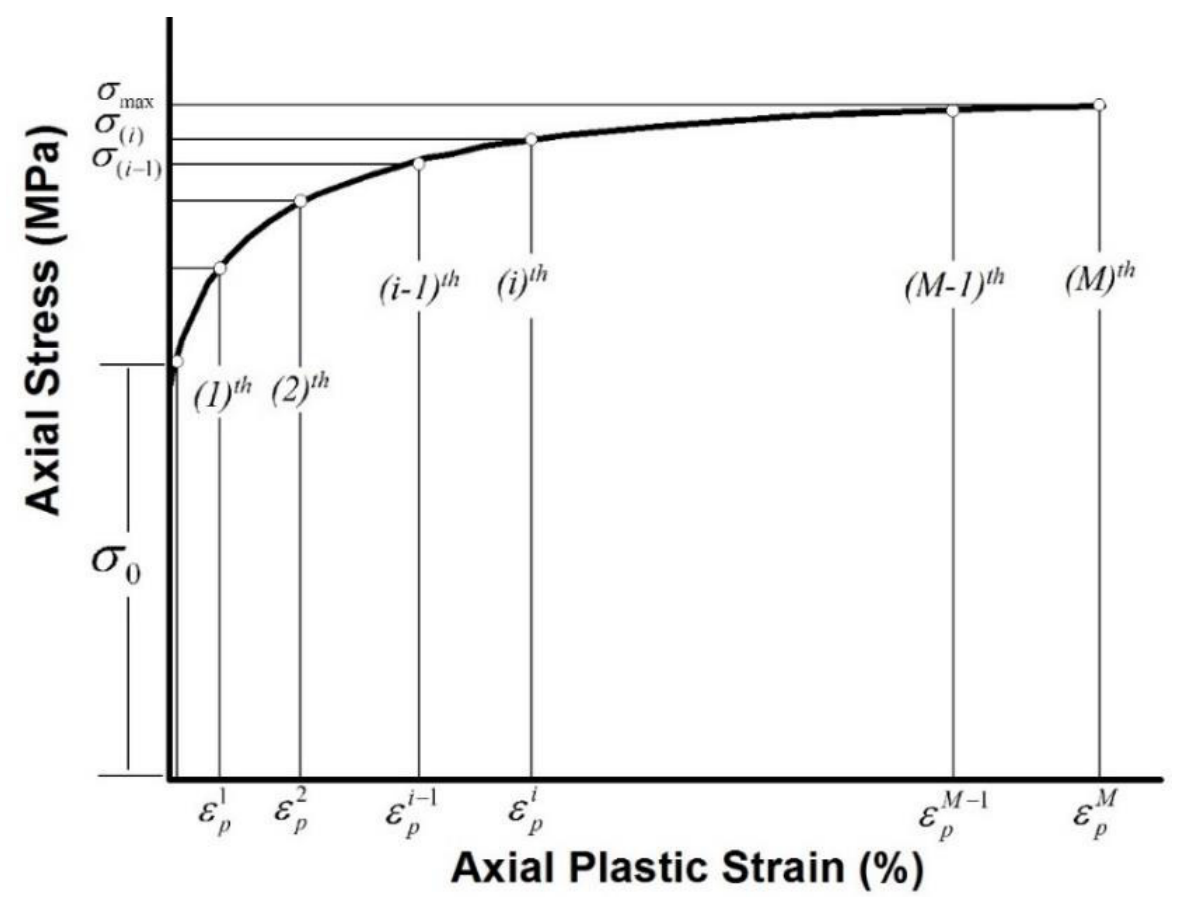

Figure 2.6 The defined segments on the uniaxial cyclic stress-strain to determine the coefficients of the $\mathrm{O}-\mathrm{W}$ hardening rule.

\subsubsection{The McDowell Model}

In the prediction of ratcheting strain based on the $\mathrm{O}-\mathrm{W}$ hardening rule, both rate and magnitude of ratcheting progress are controlled by exponents $m_{i}$. To improve the capability of the $\mathrm{O}-\mathrm{W}$ model in predicting ratcheting strain under multiaxial loading conditions, exponents $m_{i}$ were modified by McDowell [23]. The exponent $m_{i}$ in equation (2.10a) is related to noncoaxiality of the plastic strain increment and relative distance of backstress component from its origin in the form of equation (2.9b):

$$
d \bar{a}_{i}=\gamma_{i}\left[\frac{2}{3} r_{i} d \bar{\varepsilon}_{p}-\left(\frac{\left|\bar{a}_{i}\right|}{r_{i}}\right)^{m_{i}}\left\langle d \bar{\varepsilon}_{p} \cdot \frac{\bar{a}_{i}}{\left|\bar{a}_{i}\right|}\right\rangle \bar{a}_{i}\right]
$$




$$
m_{i}=A_{i}\left\langle\bar{n} \cdot \frac{\bar{a}_{i}}{\left|\bar{a}_{i}\right|}\right\rangle^{B_{i}}
$$

where $A_{i}$ is calibrated to predict the best fit for the uniaxial materials ratcheting similar to $m_{i}$ in the Ohno-Wang model (equation (2.8)) while $B_{i}$ is calibrated to fit the ratcheting response of material under multiaxial loading. The noncoaxiality reflected by $B_{i}$ was introduced to simulate the softening effects of non-proportionality. Based on the definition of $m_{i}$ the nonlinearity level of recall term decreases with increasing the relative directions of $\bar{a}_{i} /\left|\bar{a}_{i}\right|$ and $\bar{n}$. As the level of nonproportionality increases, $m_{i}$ diminishes the nonlinearity of dynamic recovery term which results in larger ratcheting strain. At $B_{i}=0$, the McDowell reduces to the $\mathrm{O}-\mathrm{W}$ hardening rule. With an increase in $B_{i}$ the McDowell model overpredicts ratcheting strain as compared to that of the O-W model.

\subsubsection{The Jiang-Sehitoglu (J-S) Model}

Jiang [6] experimentally found that evolution of backstress is associated with loading nonproportionality and that the rate of ratcheting strain is dependent on the stress level. These parameters were further employed to modify the O-W capability in ratcheting assessment of materials under non-proportional loading conditions through exponent $m_{i}[21]$. The J-S hardening rule was presented in the form of a series of backstress components as:

$$
d \bar{a}_{i}=\gamma_{i}\left[\frac{2}{3} r_{i} d \bar{\varepsilon}_{p}-\left(\frac{\left|\bar{a}_{i}\right|}{r_{i}}\right)^{m_{i}} \bar{a}_{i} d p\right]
$$

where $d p=\sqrt{d \bar{\varepsilon}_{p} \cdot d \bar{\varepsilon}_{p}}$ corresponds to the equivalent plastic strain increment. Exponent $m_{i}$ in Eq. (2.11) is defined as:

$$
m_{i}=m_{0 i}\left(2-\bar{n} \cdot \frac{\bar{a}_{i}}{\left|\bar{a}_{i}\right|}\right)
$$


where $m_{i}(i=1,2, \ldots, M)$ introduces the non-proportionality effect in ratcheting assessment of materials through term $\left(2-\bar{n} \cdot \bar{a}_{i} /\left|\bar{a}_{i}\right|\right)$. Term $\bar{n} \cdot \bar{a}_{i} /\left|\bar{a}_{i}\right|$ is referred as the non-proportionality factor. Under uniaxial loading condition, for constant value of $m_{i}$ (i.e., $m_{i}=m_{0 i}$ ) the J-S rule reduces to the $\mathrm{O}-\mathrm{W}$ model. The parameters in the O-W model can also be employed in the J-S and McDowell models and consequently, similar ratcheting strain values are achieved based on the $\mathrm{O}-\mathrm{W}, \mathrm{J}-\mathrm{S}$, and McDowell hardening rules under uniaxial loading condition. The J-S hardening rule, however showed an overestimation of ratcheting response of materials subjected to multiaxial loading conditions $[16,30]$. This model holds $d p$ in the dynamic recovery term while components $d \bar{\varepsilon}_{p}$ and $\bar{a}_{i} /\left|\bar{a}_{i}\right|$ in the MaCaulay brackets in the dynamic recovery term of the $\mathrm{O}-\mathrm{W}$ rule possess different directions under multiaxial loading. The projection presented by $\left\langle d \bar{\varepsilon}_{p} \cdot \bar{a}_{i} /\left|\bar{a}_{i}\right|\right\rangle$ in the O-W is smaller than $d p$ in the J-S rule. The O-W rule holding $\left\langle d \bar{\varepsilon}_{p} \cdot \bar{a}_{i} /\left|\bar{a}_{i}\right|\right\rangle$ shows slower evolution in ratcheting progress under multiaxial loading than the $\mathrm{J}-\mathrm{S}$ model including $d p$ in the dynamic recovery term. In the $\mathrm{O}-\mathrm{W}$ rule, the function with the MaCaulay brackets reduces to $d p$ under uniaxial loading condition. Exponent $m_{0 i}$ in the J-S hardening rule is equivalent with exponent $m_{i}$ in the $\mathrm{O}-\mathrm{W}$ hardening rule. The exponent $m_{i}$ introduced through the J-S rule resulted in larger value of $m_{i}$ as compared to that of the $\mathrm{O}-\mathrm{W}$ rule and consequently caused slower evolution in ratcheting progress under multiaxial loading conditions. This interactive effect between $m_{i}$ and $d p$ in the JS hardening rule needs to be analysed for different loading paths, which will be addressed in this study.

\subsubsection{The Chen-Jiao-Kim (C-J-K) Model}

In the O-W hardening rule, both rate and magnitude of ratcheting progress are controlled by exponents $m_{i}$. To improve the capability of the $\mathrm{O}-\mathrm{W}$ model in ratcheting assessment under multiaxial loading conditions, exponents $m_{i}$ were modified by McDowell [23] and Jiang-Sehitoglu [21]. The modifications on $m_{i}$ were yet to accurately assess ratcheting under complex multiaxial 
loading conditions. To overcome this drawback, Chen-Jiao-Kim (C-J-K) [30] further modified the O-W second model by introducing term $\left\langle\bar{n} . \bar{a}_{i} /\left|\bar{a}_{i}\right|\right\rangle$ constructed based on backstress and the noncoaxiality of the plastic strain rate:

$$
d \bar{a}_{i}=\gamma_{i}\left[\frac{2}{3} r_{i} d \bar{\varepsilon}_{p}-\left\langle\bar{n} \cdot \frac{\bar{a}_{i}}{\left|\bar{a}_{i}\right|}\right\rangle^{\chi_{i}}\left(\frac{\left|\bar{a}_{i}\right|}{r_{i}}\right)^{m_{i}}\left\langle d \bar{\varepsilon}_{p} \cdot \frac{\bar{a}_{i}}{\left|\bar{a}_{i}\right|}\right\rangle \bar{a}_{i}\right]
$$

The exponent $\chi_{i}$ in equation (2.13) is referred as multiaxial factor. Under nonproportional loading, term $\left\langle\bar{n} . \bar{a}_{i} /\left|\bar{a}_{i}\right|\right\rangle$ takes values of either zero or less than unity and corresponds to the non-proportionality factor and consequently the term in the MaCaulay brackets, $\left.\left\langle\bar{n} .\left(\bar{a}_{i} / \mid \bar{a}_{i}\right)\right\rangle\right\rangle^{\chi_{i}}$ incorporates the non-proportionality effect into the hardening rule. For $\chi_{i} \rightarrow \infty$ the C-J-K hardening rule reduces to a multilinear model experiencing premature shakedown. For $\chi_{i}=0$ the C-J-K reduces to the second $\mathrm{O}-\mathrm{W}$ model (equation (2.8)). Under uniaxial loading condition, term $\left\langle\bar{n} . \bar{a}_{i} /\left|\bar{a}_{i}\right|\right\rangle$ takes values either zero or unity resulting in an ineffective exponent $\chi_{i}$ then the $\mathrm{C}-\mathrm{J}-\mathrm{K}$ model reduces to the $\mathrm{O}-\mathrm{W}$ rule. Under multiaxial loading, the term $\left\langle\bar{n} . \bar{a}_{i} /\left|\bar{a}_{i}\right|\right\rangle$ becomes less than unity and exponent $\chi_{i}\left(0<\chi_{i}<\infty\right)$ noticeably influences the ratcheting rate and magnitude. As this exponent approaches infinity $\left(\chi_{i} \rightarrow \infty\right)$, ratcheting rate and magnitude reduces toward ratcheting arrest. The value of the exponent $\chi_{i}$ is calibrated based on the experimental ratcheting curve under multiaxial loading condition.

\subsubsection{The Ahmadzadeh-Varvani (A-V) Model}

The A-V model was developed on the basis of constitutive equations at which the calculation of the modulus of plasticity, $H_{p}$, was coupled with consistency condition of yield surface. The A-V model with its dynamic recovery term introduced a non-linear trend in the hardening rule. This hardening rule holds coefficients dictating the evolution of the yield surface 
on the deviatoric stress space to accumulate the ratcheting strain with the gradual decreasing rate. For uniaxial loading, the A-V hardening rule was expressed as [34-35, 69].

$$
\begin{aligned}
& d \bar{a}=C d \bar{\varepsilon}_{p}-\gamma_{1}(\bar{a}-\delta \bar{b}) d p \\
& d \bar{b}=\gamma_{2}(\bar{a}-\bar{b}) d p
\end{aligned}
$$

The tensor $\bar{b}$ is an additional kinematic variable with the initial value of zero. Coefficients $\mathrm{C}$ and $\gamma_{1}$ are determined from experimental stress-strain hysteresis under stress-controlled loading condition. Coefficients $\gamma_{2}$ and $\delta$ were found to be material and stress level dependent. Coefficient $\delta$ avoids ratcheting arrest after certain number of cycles and results in a constant rate followed by ratcheting strain rate decay over stage I. Coefficients $\gamma_{2}$ and $\delta$ were defined based on the material properties and stress levels. Both coefficients $\gamma_{2}$ and $\delta$ were defined for various stress amplitudes and mean stresses in non-linear forms as:

$$
\gamma_{2}=\sqrt{\sigma_{m}}\left(\sigma_{a}-\sigma_{y}\right)^{\rho_{1}}+K\left(\sigma_{a}-\sigma_{y}\right)^{\rho_{2}}
$$

and

$$
\delta=1-F e^{\lambda \sigma_{a}}
$$

Constant $F=2\left(\sigma_{y}-\sigma_{m}\right) / E$. Terms $\mathrm{E}$ and $\sigma_{\mathrm{y}}$ represent respectively modulus of elasticity and size of yield surface. In equations (2.15) and (2.16), constants $K, \rho_{1}, \rho_{2}$, and $\lambda$ are materials constants. Coefficients $\gamma_{2}$ and $\delta$ can also can be estimated using typical family curves shown in figure 2.7 for SS304 steel samples. 

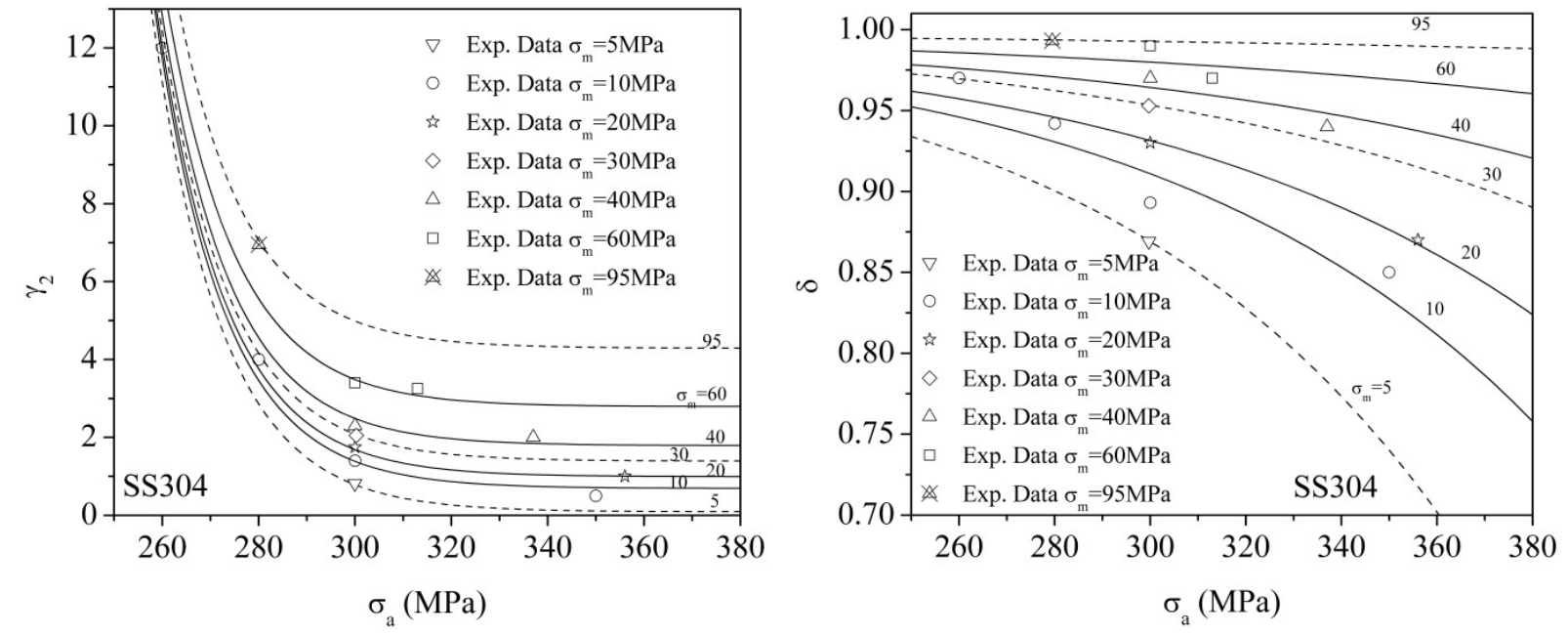

Figure 2.7 Family curves representing the variations of (a) coefficient $\gamma_{2}$ and (b) coefficient $\delta$ for different $\sigma_{\mathrm{a}}$ and $\sigma_{\mathrm{m}}$ values [34].

Coefficient $\gamma_{2}$ controls ratcheting rate and regulates the ratcheting response of materials at various loading conditions. Coefficients $\delta$ is responsible to control the accumulation rate of ratcheting strain and prevents the modified model to experience plastic shakedown as the number of cycles advances. Figure 2.8 presents the effects of $\gamma_{2}$ and $\delta$ on the trend of A-V hardening rule in the ratcheting prediction of $42 \mathrm{CrMo}$ steel samples. In figure $2.8 \mathrm{a}$, for $\delta=1$ the model reduces to the Bower's model experiencing plastic shakedown and for $\delta=0$ the model acts as the A-F model predicting same hysteresis loops over stress cycles which results in a constant rate of ratcheting strain with no decay in the accumulation rate of ratcheting strain. In figure $2.8 \mathrm{~b}$, for $\gamma_{2}=0$ the A-V model reduces to A-F model. In case of $\gamma_{1}=\gamma_{2}=0$ the model converts to linear hardening rule of Prager's. Coefficients $\gamma_{2}$ and $\delta$ compromise the overestimation of A-F rule and the plastic shakedown predicted by the Bower hardening rule. 

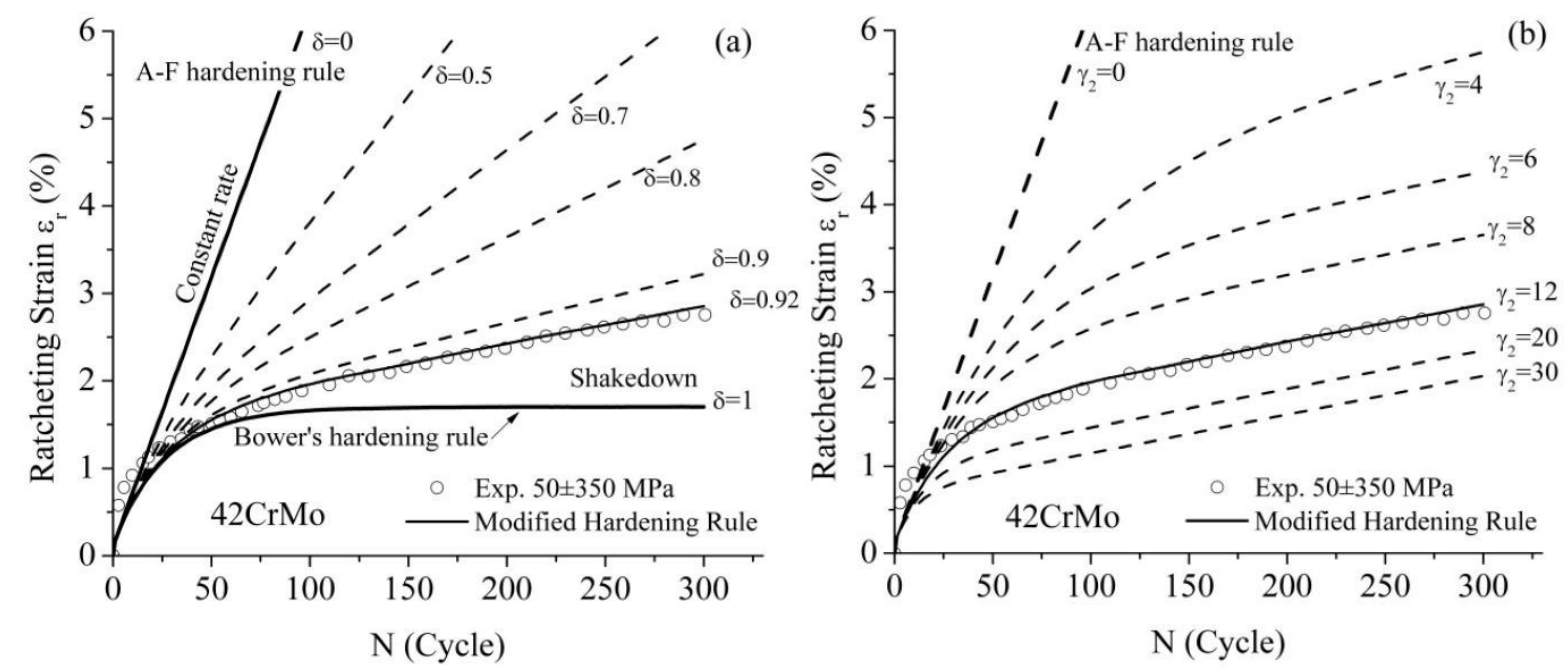

Figure 2.8 The variation of coefficients $\delta$ and $\gamma_{2}$ on the ratcheting strain over stress cycles [34].

\subsection{Summary}

The framework of several coupled kinematic hardening rules were described and studied. The linear hardening rule of Prager failed to produce open hysteresis loops and accumulate ratcheting strain. The A-F mode predicted open hysteresis loops and ratcheting over stress cycles. The rate of ratcheting progress stayed constant and resulted in a large overestimation based on the A-F model as compared with experimental ratcheting data. The A-F hardening rule was further modified by Bower through inclusion of an additional kinematic variable $\bar{b}$ in the dynamic recovery term to introduce the ratcheting rate decay. Despite this breakthrough, Bower's model experiences plastic shakedown over the second stage. To overcome this drawback, Ahmadzadeh and Varvani offered a hardening rule holding coefficients $\gamma_{2}$ and $\delta$ in the dynamic recovery term to compromise the overestimation of A-F model and the plastic shakedown predicted by Bower's rule. Ohno and Wang developed a kinematic hardening rule based on the critical state of dynamic recovery in backstress equation. The total backstress was consequently determined by the superposition of $M$ independent backstress components in this model. Attempts were made by McDowell, Jiang-Sehitoglu and Chen-Jiao-Kim to further modify the O-W model by inclusion of non-proportionality effects into the framework of this model. 


\section{CHAPTER THREE}

\section{FUNDAMENTAL ELEMENTS OF THE PROPOSED HARDENING RULE}

This chapter presents the constitutive equations to assess ratcheting response of materials over stress cycles. The framework and components of cyclic plasticity are defined and the evolutional modifications made on the $\mathrm{A}-\mathrm{V}$ hardening rule are presented to finally introduce the modified hardening rule. The influential elements in the dynamic recovery term of the modified model including $\gamma_{2},\left\langle d \bar{\varepsilon}_{p} \cdot \bar{a} /|\bar{a}|\right\rangle,\langle\bar{n} \cdot \bar{a} /|\bar{a}|\rangle^{1 / 2}$ and $(2-\bar{n} \cdot \bar{a} /|\bar{a}|)$ terms are defined. The step-wise procedures for calculation of modulus of plasticity and unknown elements in case of applied mixed stress- strain controlled multiaxial conditions are presented. The algorithm of the modified hardening rule is detailed in this chapter and the MATLAB programming code is presented in Appendix A.

\subsection{Cyclic plasticity and A-F type kinematic hardening rules}

\subsubsection{Framework of cyclic plasticity}

The backbone of cyclic plasticity models is structured by important constituents of strain increment, Hooke's law, yield function, flow rule, hardening rule and consistency condition. Materials are assumed to present elastic behaviour with strain-stress relation without plastic strain until the yield condition is satisfied. The von Mises criterion is given as:

$$
f\left(\bar{s}, \bar{a}, \sigma_{y}\right)=\frac{3}{2}(\bar{s}-\bar{a}) \cdot(\bar{s}-\bar{a})-\sigma_{y}^{2}=0
$$


where $\bar{s}$ is the deviatoric stress tensor and defined as:

$$
\bar{s}=\bar{\sigma}-\frac{1}{3}(\bar{\sigma} \cdot \bar{I}) \bar{I}
$$

During loading, the size and shape of yield surface remain unchanged. The yield surface can translate in the deviatoric stress space. Total strain increment is composed of both elastic and plastic strain components as:

$$
d \bar{\varepsilon}=d \bar{\varepsilon}^{e}+d \bar{\varepsilon}^{p}
$$

Elastic strain is defined by Hooke's law as:

$$
\bar{\varepsilon}^{e}=\frac{\bar{\sigma}}{2 G}-\frac{v}{E}(\bar{\sigma} \cdot \bar{I}) \bar{I}
$$

where terms $\bar{I}$ and $\bar{\sigma}$ correspond respectively to unit and stress tensors. Elastic and plastic regions is partitioned by employing von Mises criterion and the plastic strain increment is obtained based on the associated flow rule which was first proposed by Drucker [70-71] to define the relation of stress and strain increments as:

$$
d \bar{\varepsilon}^{p}=\frac{1}{H_{p}}(d \bar{s} \cdot \bar{n}) \bar{n}
$$

where the normal vector to the yield surface is defined as:

$$
\bar{n}=\frac{\bar{s}-\bar{a}}{|\bar{s}-\bar{a}|}
$$

Terms $H_{p}$ and $d \bar{s}$ are the plastic modulus and the increment of deviatoric stress tensor, respectively.

During an elastic-plastic deformation loading condition the consistency condition of yield surface needs to be satisfied. The consistency condition implies for the same projection of 
backstress and stress state increments on the unit exterior normal $\bar{n}$ during an elastic-plastic loading condition and is defines as:

$$
d \bar{a} \cdot \bar{n}=d \bar{s} \cdot \bar{n}
$$

The plastic modulus $H_{p}$ can be derived through the equations of flow rule (equation (3.5)), the consistency condition of yield surface (equation (3.7)), and a kinematic hardening rule as the central part of cyclic plasticity theory. The hardening rule defines the movement direction of yield surface in the stress space during plastic deformation.

\subsubsection{A-F type kinematic hardening rules}

Recalling from Chapter 2, Armstrong and Frederick [13] modified Prager's model [15] by adding a "dynamic recovery" term introducing nonlinearity to A-F model:

$$
d \bar{a}=C d \bar{\varepsilon}_{p}-\gamma \bar{a} d p
$$

where

$$
d p=\sqrt{d \bar{\varepsilon}_{p} \cdot d \bar{\varepsilon}_{p}}
$$

Coefficients $\mathrm{C}$ and $\gamma$ are material dependent and determined using strain-stress hysteresis loop obtained under strain-controlled loading condition. The second term in equation (2.2) introducing nonlinearity to the model is responsible for the accumulation of plastic strain over stress cycles. The A-F hardening rule predicts same loops over all stress cycles and results in a constant rate of ratcheting strain and consequently the overestimation of ratcheting strain.

Bower [15] further modified the A-F rule to overcome this drawback. He introduced the second kinematic variable $\gamma_{2}$ into the dynamic recovery term to reduce the constant rate of strain accumulation predicted by the A-F hardening rule as the number of stress cycles advances. The 
Bower's model defined in equation (2.5) employs the second kinematic variable in the recall term to simulate the accumulating ratcheting strain decay:

$$
\begin{aligned}
& d \bar{a}=C d \bar{\varepsilon}_{p}-\gamma_{1}(\bar{a}-\bar{b}) d p \\
& d \bar{b}=\gamma_{2}(\bar{a}-\bar{b}) d p
\end{aligned}
$$

Bower model is capable to simulate rate decay in predicting the ratcheting strain over initial number of cycles and over stage I. However, this model shows premature shakedown in stage II.

Ahmadzadeh-Varvani [34, 69] further modified Bower's hardening rule to encounter the influence of stress levels over ratcheting stages by means of $\gamma_{2}$ and $\delta$ coefficients introduced into the dynamic recovery term of the hardening rule. The A-V hardening rule was then described in the form of:

$$
\begin{aligned}
& d \bar{a}=C d \bar{\varepsilon}_{p}-\gamma_{1}(\bar{a}-\delta \bar{b}) d p \\
& d \bar{b}=\gamma_{2}(\bar{a}-\bar{b}) d p
\end{aligned}
$$

The tensor $\bar{b}$ is an additional kinematic variable with the initial value of zero. Both $C$ and $\gamma_{1}$ in the A-V hardening rule (Eq. (2.14.)) are material dependent coefficients and responsible to control the width of stress-strain hysteresis loops over ratcheting progress. Coefficients $\mathrm{C}$ and $\gamma_{1}$ are determined from experimental stress-strain hysteresis under stress-controlled loading condition. Coefficients $\gamma_{2}$ and $\delta$ were defined to be material and stress level dependent through the equations (2.15) and (2.16). Coefficients $\gamma_{2}$ and $\delta$ can also be estimated using corresponding family curves with respect to $\sigma_{\mathrm{a}}$ and $\sigma_{\mathrm{m}}$.

\subsection{Modifications on the $\mathrm{A}-\mathrm{V}$ hardening rule and dynamic recovery term}

Components undergoing multiaxial stress cycles and various non-proportional loading histories are more prone to hardening than those of uniaxially and proportionally loaded. 
Modifications have been made to extend the capability of the A-V hardening rule to predict multiaxial ratcheting response of materials under various loading paths. The modified hardening rule holding function $\left\langle d \bar{\varepsilon}_{p} \cdot \bar{a}|| \bar{a} \mid\right\rangle$ in dynamic recovery term is capable to assess ratcheting at different directions under multiaxial loading conditions due to components $d \bar{\varepsilon}_{p}$ and $\bar{a} /|\bar{a}|$. The projection presented by the function in the MaCaulay brackets is smaller than $d p$ (in equation (2.14)) resulting in a slower evolution in simulation of ratcheting strain under multiaxial loading conditions. The calibrating coefficient $\gamma_{2}$ in the modified model is redefined to be merely material dependent. The $\mathrm{A}-\mathrm{V}$ hardening rule was further modified by introducing the term $(\bar{n} \cdot \bar{a} /|\bar{a}|)$ into the dynamic recovery term to encounter the effect of non-proportionality and structured as:

$$
\begin{aligned}
& d \bar{a}=C d \bar{\varepsilon}_{p}-\gamma_{1}\left(\bar{a}-\left\langle\bar{n} \cdot \frac{\bar{a}}{|\bar{a}|}\right\rangle^{1 / 2} \bar{b}\right)\left\langle d \bar{\varepsilon}_{p} \cdot \frac{\bar{a}}{|\bar{a}|}\right\rangle, \\
& d \bar{b}=\left(2-\bar{n} \cdot \frac{\bar{a}}{|\bar{a}|}\right) \gamma_{2}(\bar{a}-\bar{b})\left\langle d \bar{\varepsilon}_{p} \cdot \frac{\bar{a}}{|\bar{a}|}\right\rangle .
\end{aligned}
$$

In equation (3.8), term $(\bar{n} . \bar{a} /|\bar{a}|)$ holds non-proportional characteristics under multiaxial loading conditions. The normal vector to the yield surface $\bar{n}$ and the backstress unit vector $\bar{a} /|\bar{a}|$ have different directions under multiaxial loading, and consequently the projection presented by $(\bar{n} . \bar{a} /|\bar{a}|)$ produces values between -1 and 1 , however in uniaxial cases, this term is unity. The term $(2-\bar{n} \cdot \bar{a} /|\bar{a}|)$ regulates the magnitude of coefficient $\gamma_{2}$ properly for various multiaxial loading paths. This term takes the values between 1 and 3 under multiaxial loading and consequently magnifies the effect of $\gamma_{2}$ and results in a smaller accumulation rate of ratcheting strain over stress cycles. Term $(2-\bar{n} \cdot \bar{a} /|\bar{a}|)$ encounters the effect of non-proportionality as multiaxial loads are applied and reduces to unity for uniaxial loading condition. For $\gamma_{2}=0$, the modified model reduces to A-F model. In case of $\gamma_{1}=\gamma_{2}=0$ the proposed model converts to linear hardening rule of Prager's. The measure of non-proportionality was also utilized in the form of 
product of $\langle\bar{n} \cdot \bar{a} /|\bar{a}|\rangle^{1 / 2}$ and vector $\bar{b}$ to control the accumulation rate of ratcheting strain and mainly to prevent the modified model to experience plastic shakedown as the number of cycles advances over various multiaxial loading conditions. Under multiaxial loading histories, term $\langle\bar{n} . \bar{a} /|\bar{a}|\rangle^{1 / 2}$ takes positive values smaller than 1 and prevents this model from premature plastic shakedown. For uniaxial loading, since term $\langle\bar{n} \cdot \bar{a} /|\bar{a}|\rangle^{1 / 2}$ is equal to 1 , the modified model reduces to Bower's model.

Figure 3.1 presents the evolution of the modified hardening rule (Eqn (3.8)) for typical ratcheting assessment of 1045 steel samples for cases at which (i) the accumulated plastic strain increment $d p$ is replaced with $\left\langle d \bar{\varepsilon}_{p} \cdot \bar{a} /|\bar{a}|\right\rangle$ in the dynamic recovery term of equation (3.8a), (ii) the term $\langle\bar{n} \cdot \bar{a} /|\bar{a}|\rangle^{1 / 2}$ in equation (3.8a) is absent, (iii) the term $(2-\bar{n} \cdot \bar{a} /|\bar{a}|)$ in equation (3.8b) is absent, and (iv) the modified hardening rule holding all terms of equation (3.8). 


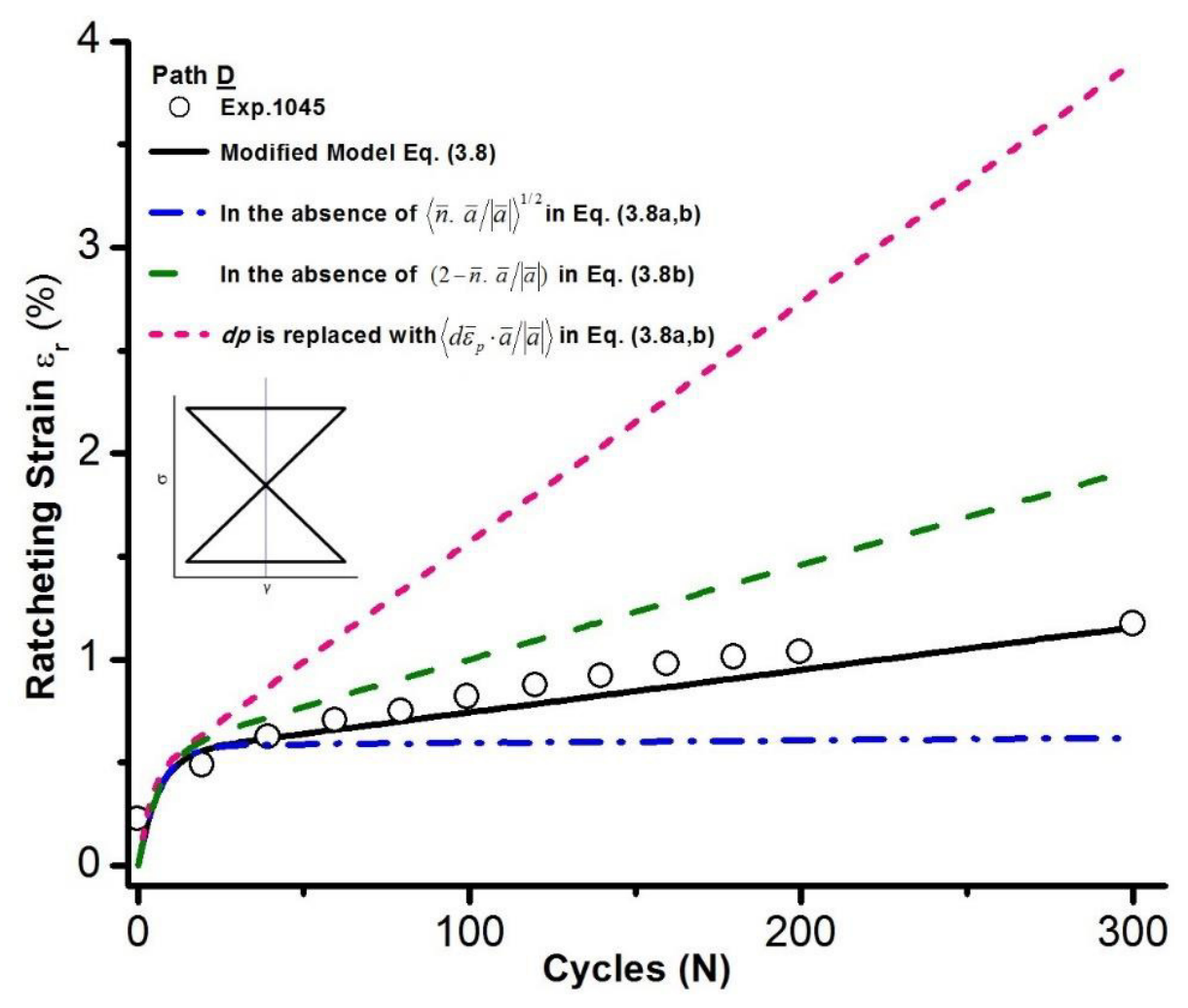

Figure 3.1 Experimental data [30] and predicted ratcheting strain values of 1045 steel samples for butterfly loading path based on the modified model.

\subsubsection{Estimation of $C, \gamma_{1}$ and $\gamma_{2}$ coefficients}

Coefficients $\mathrm{C}$ and $\gamma_{1}$ in the A-V and modified hardening rules are defined and calibrated on the basis of strain-stress hysteresis loops over stress cycles. In the A-F model [13], constants C and $\gamma_{1}$ are determined from the uniaxial stress-strain hysteresis loop under strain-controlled condition. The material constants $\mathrm{C}$ and $\gamma_{1}$ in the Bower [15] hardening rule were obtained from the unsymmetrical uniaxial stress-strain hysteresis loops. The $\gamma_{2}$ in this model, is also chosen to give the correct total accumulated strain after $\mathrm{N}_{1}$ and $\mathrm{N}_{2}$ stress cycles. Constants $m_{i}, r_{i}$ and $\gamma_{i}$ in the kinematic hardening rules of Jiang-Sehitoglu [6], Ohno-Wang [19, 20] and Chen et al. [30] are defined by segments chosen on the uniaxial stress-strain and uniaxial ratcheting curves through a tedious and time-consuming trial procedure, which remain constant after determination during each increment of cyclic plasticity theory. These constants are highly affected by the chosen segments over stress-strain curve. 
Material dependent coefficients $\mathrm{C}$ and $\gamma_{1}$ are estimated to properly coincide predicted and experimental stress-strain hysteresis loops while satisfying the consistency condition of yield surface in forward and reversed loading [35,69]. Both $\mathrm{C}$ and $\gamma_{1}$ coefficients are material dependent coefficients to control the shape and size of stress-strain hysteresis loops over evolution of ratcheting strain. Figure $3.2 \mathrm{~b}$ shows how influential the effects of different values of $\mathrm{C}$ and $\gamma_{1}$ on the shape and size of hysteresis loops of SS304 under asymmetrical uniaxial loading are. Various combinations of $\mathrm{C}$ and $\gamma_{1}$ values are examined in a closed form solution of the proposed model in each individual iteration to address hysteresis loops with consistent size and shape. Figures 3.2c and 3.3d with the respective combinations of $\left(C=10 \mathrm{GPa}, \gamma_{1}=150\right),\left(\mathrm{C}=40 \mathrm{GPa}, \gamma_{1}=450\right)$ which result in the violation of consistency condition and consequently the distortion of hysteresis loops while the coefficients $\mathrm{C}=27 \mathrm{GPa}, \gamma_{1}=180$ for this material satisfied the consistency condition and resulted in progressive hysteresis loops with the number of cycles [35]. 

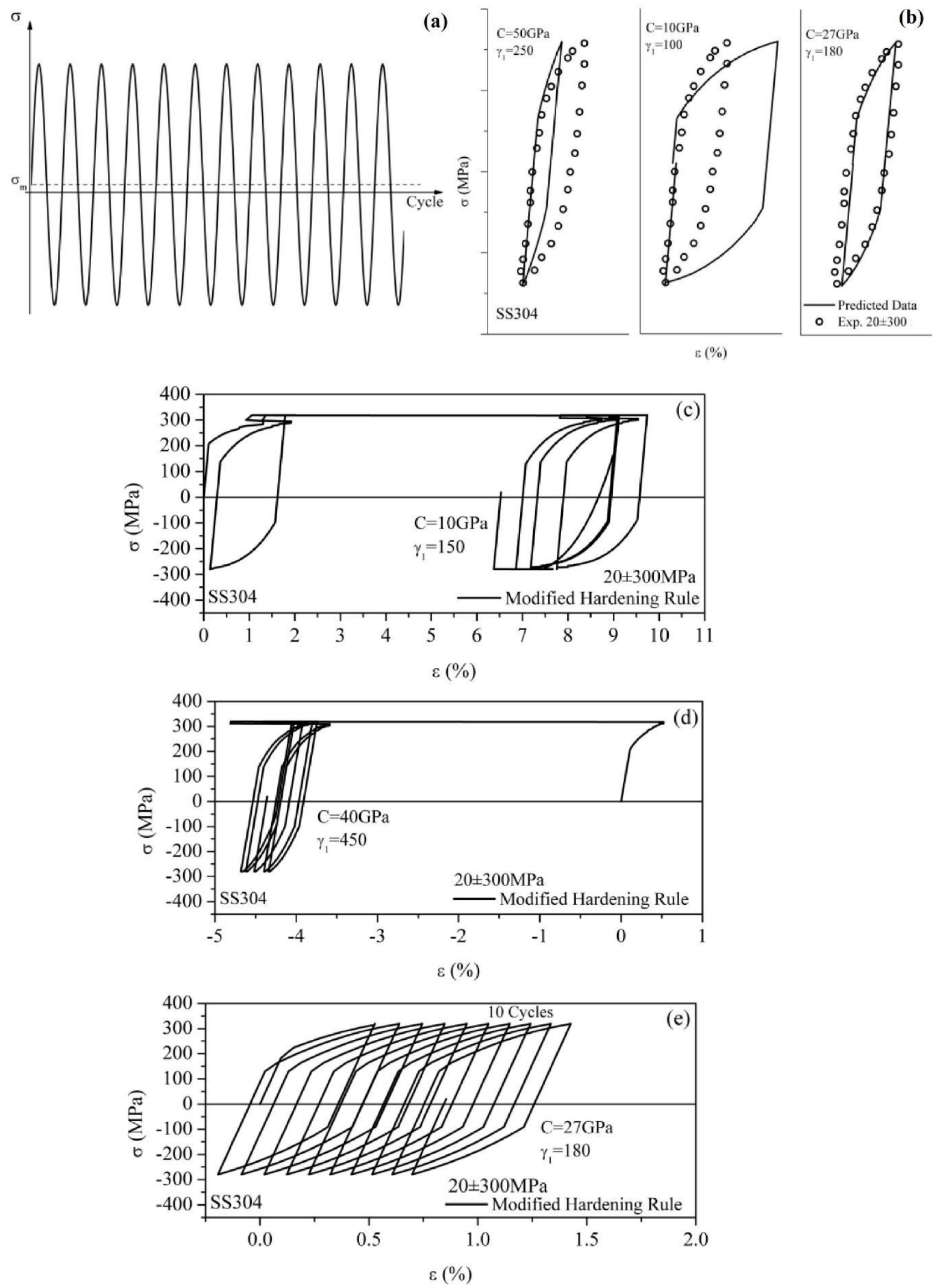

Figure 3.2 The effect of $\mathrm{C}$ and $\gamma_{1}$ on the consistency condition and hysteresis loops [35]. 
The calibrating coefficient $\gamma_{2}$ is material dependent and is determined using the ratcheting data obtained under uniaxial loading condition. Figure 3.3 shows the effect of $\gamma_{2}$ on the ratcheting assessment of SS304L under uniaxial loading of 50 \pm 200 . Coefficient $\gamma_{2}$ controls ratcheting rate and regulates the ratcheting response of materials.

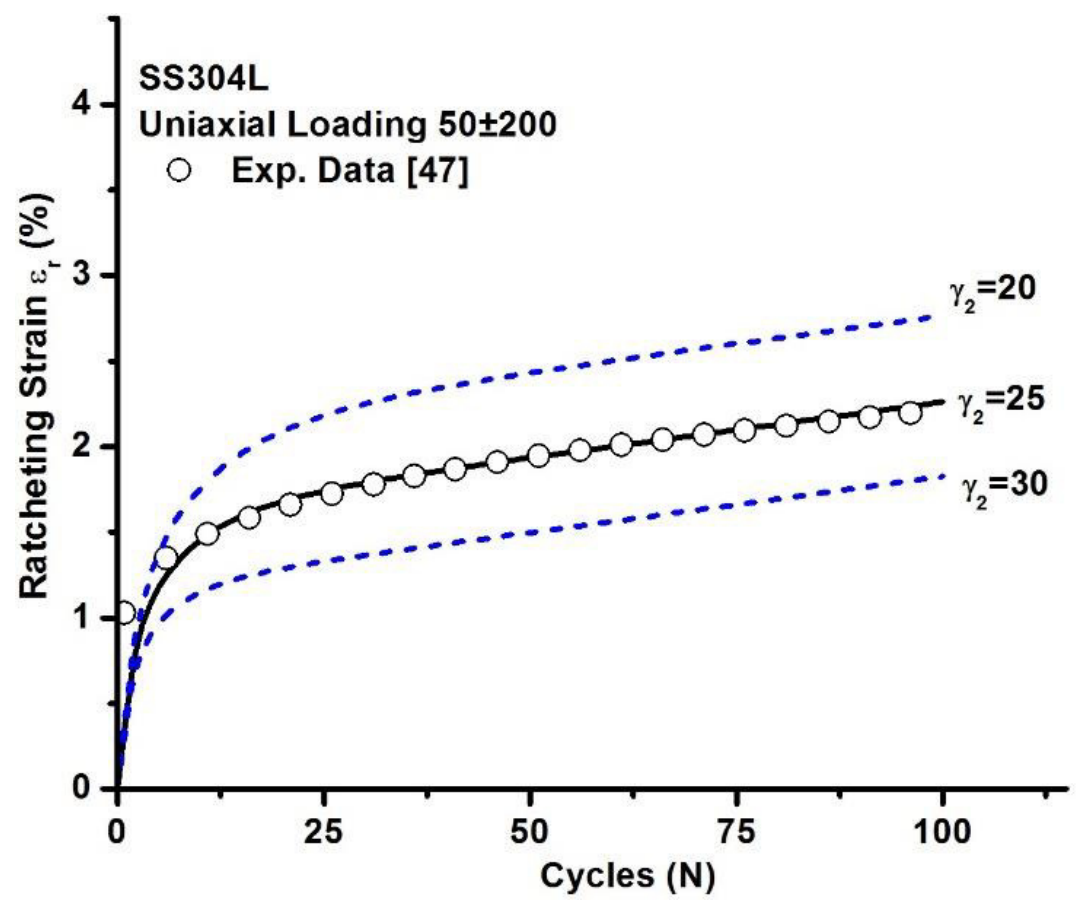

Figure 3.3 Determination of coefficient $\gamma_{2}$ in the modified model using uniaxial ratcheting response.

Under multiaxial loading, coefficient $\gamma_{2}$ non-proportionally is regulated by $(2-\bar{n} \cdot \bar{a} /|\bar{a}|)$ in the dynamic recovery term resulting in more controllable ratcheting rate over stages I and II. The term $\bar{n} \cdot \bar{a} /|\bar{a}|$ further encounters the non-proportionality in the model through $\langle\bar{n} \cdot \bar{a} /|\bar{a}|\rangle^{1 / 2}$ over asymmetric multiaxial stress cycles.

\subsubsection{Derivation of modulus of plasticity}

In the category of coupled hardening rules, the calculation of modulus of plasticity, $H p$, is coupled with the kinematic hardening rule through the consistency condition of the yield surface. 
The derivation of modulus of plasticity can be expressed in three distinctive steps using kinematic hardening rule, flow rule and the consistency condition.

(i) The definition of $d \bar{\varepsilon}^{p}$ (equation (3.9)) is substituted into the modified hardening rule (equation (3.8a)) and backstress increment $d \bar{a}_{i}$ is defined in equation (3.10):

$$
\begin{aligned}
& d \bar{\varepsilon}^{p}=d p \cdot \bar{n} \\
& d \bar{a}=C d p \bar{n}-\gamma_{1}\left(\bar{a}-\langle\bar{n} \cdot \mid \bar{a}\rangle^{1 / 2} \bar{b}\right)\left\langle d p \bar{n} \cdot \frac{\bar{a}}{|\bar{a}|}\right\rangle
\end{aligned}
$$

(ii) Based on consistency condition, the stress state during the elastic-plastic deformation should always lie on yield surface. Equation (3.7) is then extended as:

$$
d \bar{s} \cdot \bar{n}=C d p \bar{n} \cdot \bar{n}-\left[\gamma_{1}\left(\bar{a}-\left\langle\bar{n} \cdot \frac{\bar{a}}{|\bar{a}|}\right\rangle^{1 / 2} \bar{b}\right)\left\langle d p \bar{n} \cdot \frac{\bar{a}}{|\bar{a}|}\right\rangle\right] \cdot \bar{n}
$$

(iii) Plastic strain increment $d \bar{\varepsilon}^{p}$ of equation is redefined as the plastic modulus defined based on (3.9) and $d \bar{s} \cdot \bar{n}$ defined based on (3.11) are substituted into the flow rule equation (3.5):

$$
d p \cdot \bar{n}=\frac{1}{H_{p}}\left(C d p \bar{n} \cdot \bar{n}-\left[\gamma_{1}\left(\bar{a}-\left\langle\bar{n} \cdot \frac{\bar{a}}{|\bar{a}|}\right\rangle^{1 / 2} \bar{b}\right)\left\langle d p \bar{n} \cdot \frac{\bar{a}}{|\bar{a}|}\right\rangle\right] \cdot \bar{n}\right) \bar{n}
$$

The plastic modulus $H_{p}$ is then derived as:

$$
H_{p}=C-\gamma_{1}\left(\bar{a}-\left\langle\bar{n} \cdot \frac{\bar{a}}{|\bar{a}|}\right\rangle^{1 / 2} \bar{b}\right)\left\langle\bar{n} \cdot \frac{\bar{a}}{|\bar{a}|}\right\rangle \bar{n}
$$




\subsection{Algorithm of ratcheting assessment under various multiaxial loading conditions}

The multiaxial ratcheting assessment of materials are generally conducted in (i) stress-based multiaxial state, and (ii) stress- and strain-based multiaxial state. In the first case, upon the application of incremental cyclic load all the elements of applied stress tensor is known and the incremental ratcheting strain values are defined using Hooke's law and flow rule through the framework of operated kinematic hardening rule. In the second case embracing higher complexity, both stress-controlled and strain-controlled cycles are however responsible for ratcheting of materials. The present study has first transformed applied cyclic stress and strain values on the tubular steel samples to the stress components in the stress tensor over each increment. The incremental ratcheting strain values were defined using Hooke's law and flow rule through the framework of the modified hardening rule.

Under such loading conditions, incremental strain and stress respectively were defined through $d \varepsilon_{i j}^{t}=\left[\begin{array}{lll}d \varepsilon_{x x}^{t} & d \varepsilon_{x y}^{t} & d \varepsilon_{x z}^{t} \\ d \varepsilon_{y x}^{t} & d \varepsilon_{y y}^{t} & d \varepsilon_{y z}^{t} \\ d \varepsilon_{z x}^{t} & d \varepsilon_{z y}^{t} & d \varepsilon_{z z}^{t}\end{array}\right]$ and $d \sigma_{i j}=\left[\begin{array}{ccc}d \sigma_{x x} & d \sigma_{x y} & d \sigma_{x z} \\ d \sigma_{y x} & d \sigma_{y y} & d \sigma_{y z} \\ d \sigma_{z x} & d \sigma_{z y} & d \sigma_{z z}\end{array}\right]$.

Unknown elements of strain and stress increments, were studied in this research are $d \sigma_{x y}$, $d \varepsilon_{x x}^{t}, d \varepsilon_{y y}^{t}$ and $d \varepsilon_{z z}^{t}$. Thess unknown elements under multiaxial loading conditions were first symbolically defined by solving equations (3.3-3.5) simultaneously, using Symbolic Math Toolbox in MATLAB software and re-expressed by equations (3.14)-(3.17):

$$
\begin{aligned}
& d \sigma_{x y}=\frac{2 G\left(3 d \varepsilon_{x y}^{t} H_{p}-2 d \sigma_{x x} n_{x x} n_{x y}+d \sigma_{x x} n_{x y} n_{y y}+d \sigma_{x x} n_{x y} n_{z z}\right)}{3\left(4 G n_{x y}^{2}+H_{p}\right)} \\
& d \varepsilon_{x x}^{t}=\frac{3 H_{p} d \sigma_{x x}+2 E d \sigma_{x x} n_{x x}^{2}+12 G d \sigma_{x x} n_{x y}^{2}-E d \sigma_{x x} n_{x x} n_{x y}-E d \sigma_{x x} n_{x x} n_{z z}+12 G E d \varepsilon_{x y}^{t} n_{x x} n_{z z}}{3 E\left(4 G n_{x y}^{2}+H_{p}\right)}
\end{aligned}
$$


$d \varepsilon_{y y}^{t}=\frac{3 H_{p} d \sigma_{x x} v+E d \sigma_{x x} n_{y y}^{2}+12 G d \sigma_{x x} v n_{x y}^{2}-2 E d \sigma_{x x} n_{x x} n_{y y}+E d \sigma_{x x} n_{y y} n_{z z}-12 G E d \varepsilon_{x y}^{t} n_{y y} n_{x y}}{3 E\left(4 G n_{x y}^{2}+H_{p}\right)}$

$d \varepsilon_{z z}^{t}=\frac{3 H_{p} d \sigma_{x x} v+E d \sigma_{x x} n_{z z}^{2}+12 G d \sigma_{x x} v n_{x y}^{2}-2 E d \sigma_{x x} n_{x x} n_{z z}+E d \sigma_{x x} n_{y y} n_{z z}-12 G E d \varepsilon_{x y}^{t} n_{z z} n_{x y}}{3 E\left(4 G n_{x y}^{2}+H_{p}\right)}$

To evaluate ratcheting response of materials based of the modified hardening model upon the application of a stress-controlled and mixed stress- strain- controlled loading conditions in the framework of theory of advanced cyclic plasticity, a MATLAB programming was developed. The algorithm of developed MATLAB programming is presented in figure. 3.4 and includes the main components of cyclic plasticity theory and evolution of ratcheting strain over strain cycles developed for the modified hardening rule. Dashed box in the algorithm corresponds to a subroutine to address ratcheting assessment under combined stress-/strain-controlled condition tests. MATLAB codes are presented in Appendix A. 


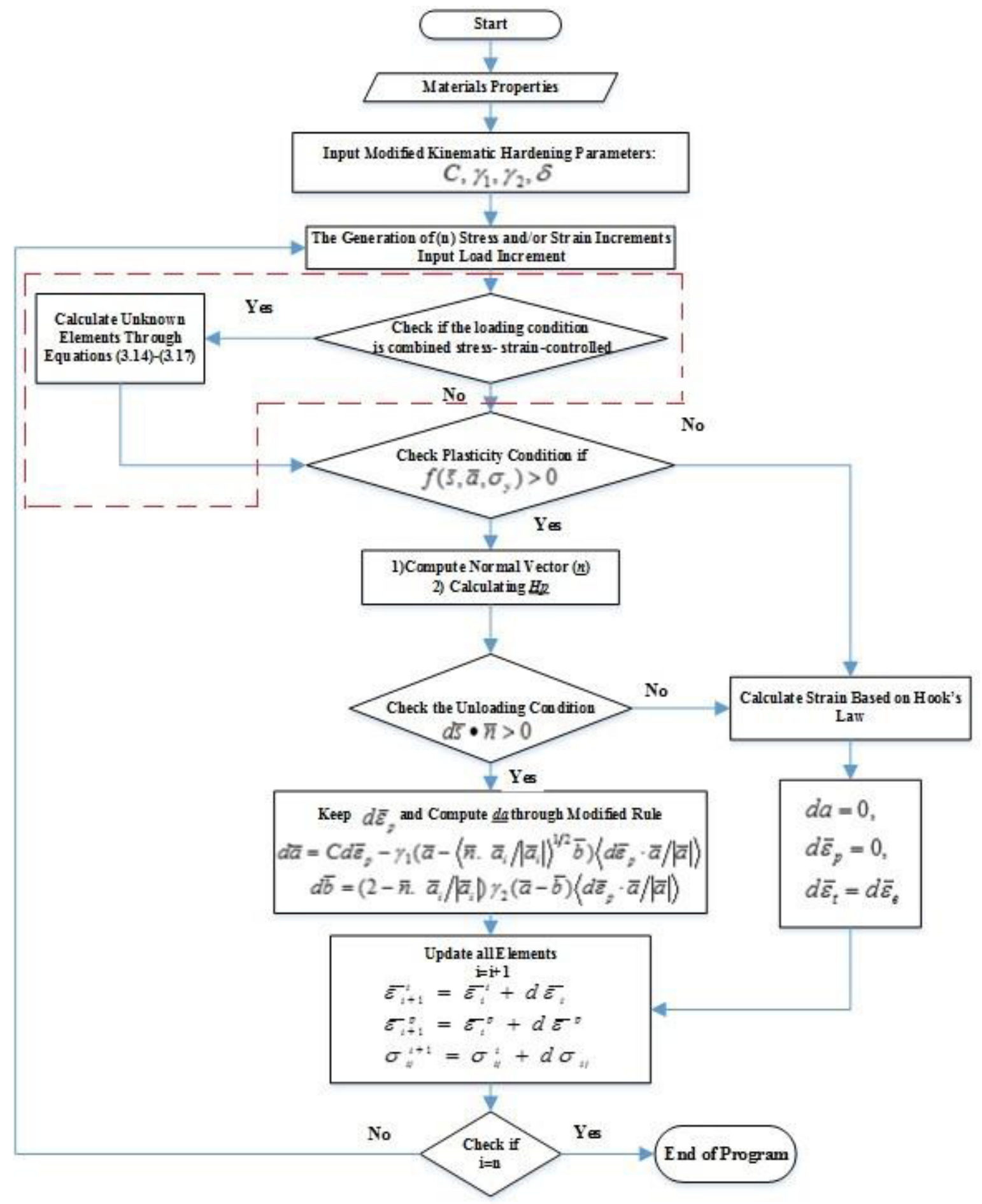

Figure 3.4 Algorithm for multiaxial ratcheting assessment based on the modified hardening rule. 


\subsection{Summary}

The A-V hardening rule is further modified through its linear and dynamic recovery terms. The linear term of backstress holds material dependent coefficients of $C$ and $\gamma_{1}$ controlling shape and size of hysteresis loops while the dynamic recovery term possesses coefficient $\gamma_{2}$ to control the ratcheting strain rate as the number of cycles advances. The plastic strain increment $d \bar{\varepsilon}_{p}$ and the backstress unity vector $\bar{a} /|\bar{a}|$ as components in the MaCaulay brackets $\left\langle d \bar{\varepsilon}_{p} \cdot \bar{a} /|\bar{a}|\right\rangle$ possessed different directions enabling the hardening rule to track reversed ratcheting during multiaxial stress cycles. Coefficient $\gamma_{2}$ is redefined in the modified model to be merely material dependent and it is determined using the ratcheting data under uniaxial loading condition. This constant is regulated by $(2-\bar{n} \cdot \bar{a} /|\bar{a}|)$ to account for non-proportionality resulting in slower ratcheting rate. Function $\langle\bar{n} \cdot \bar{a} /|\bar{a}|\rangle^{1 / 2}$ in the dynamic recovery term prevented the modified model to plastically shakedown. These terms holding backstress unity vector $\bar{a} /|\bar{a}|$ are coupled to control ratcheting rate and direction over non-proportional loading conditions. The algorithm for the modified hardening rule is developed to evaluate multiaxial ratcheting under stress-controlled and mixed stress- straincontrolled loading conditions. The MATLAB programming code for this algorithm is detailed in Appendix A. 


\section{CHAPTER FOUR}

\section{RESULTS OF RATCHETING ASSESSMENT AND DISCUSSION}

This chapter presents the results of ratcheting assessment under various multiaxial loading paths by mean of the modified hardening rule and other well-known hardening rules of O-W, J-S, and C-J-K for several steel alloys. The predicted ratcheting results by the hardening rules are compared with experimentally obtained ratcheting data in this chapter. . Materials properties and experimental ratcheting data are tabulated in Appendix B.

\subsection{The evolution of the hardening rule in ratcheting assessment of 1045 steel alloy under multiaxial loading histories}

The Ahmadzadeh-Varvani (A-V) kinematic hardening rule is further modified to assess ratcheting response of steel alloys under various multiaxial loading paths. The modified hardening rule (equation (3.8)) offered a simple framework to predict ratcheting strain over multiaxial loading cycles mainly due to modifications in the dynamic recovery term. The plastic strain increment $d \bar{\varepsilon}_{p}$ and the backstress unit vector $\bar{a} /|\bar{a}|$ as components in the MaCaulay brackets $\left\langle d \bar{\varepsilon}_{p} \cdot \bar{a} /|\bar{a}|\right\rangle$ possessed different directions enabling the hardening rule to track reversed ratcheting during multiaxial stress cycles. The calibrating coefficient $\gamma_{2}$ in dynamic recovery term controlled the ratcheting rate and was regulated by term $(2-\bar{n} \cdot \bar{a} /|\bar{a}|)$ accounting for non-proportionality to further lower the ratcheting strain curve. Function $\langle\bar{n} \cdot \bar{a} /|\bar{a}|\rangle^{1 / 2}$ in the dynamic recovery term prevented the modified model to plastically shakedown. These terms holding backstress unit vector 
$\bar{a} /|\bar{a}|$ and the normal vector $\bar{n}$ to the yield surface are coupled to control ratcheting rate and direction over multiaxial stress cycles under non-proportional loading conditions.

\subsubsection{5 steel samples, testing and multiaxial ratcheting data}

To evaluate the capability of the modified hardening rule in ratcheting assessment of materials, eight data tests conducted on 1045 steel alloy at various multiaxial loading paths were extracted from literature [30]. Ratcheting tests on 1045 steel samples under multiaxial loading conditions were conducted at which both stress-controlled and strain-controlled cycles were responsible for ratcheting of steel samples.

The tubular test samples of 1045 with inside and outside diameters of $10 \mathrm{~mm}$ and $12.5 \mathrm{~mm}$ in the gauge section, were cyclically loaded using tension-torsion loading conditions using an Instron testing machine. Extensometers were mounted on the outside of the sample gauge section to measure plastic strain over stress cycles with the frequency of $0.5 \mathrm{~Hz}$ at room temperature. The tests conducted using triangular waveform. The different multiaxial and loading paths conducted on samples of 1045 are presented in Fig. 4.1. In eight loading histories, the amplitude of shear strain was $0.866 \%$ and major ratcheting strain accumulation occurs in axial direction. Table 4.1

presents ratcheting tests and loading conditions for tubular 1045 steel samples. In figure 4.1, loading path A corresponds to shear strain cycle applied concurrently with an axial constant stress. Path B presents a shear strain cycle and an axial stress with a mean value of $50 \mathrm{MPa}$. Load path C is diamond-shaped with a fully reversed shear strain and axial stress consisted of mean value of 50MPa. Load path D vertical butterfly and paths E, F, G, and $\mathrm{H}$ are horizontal butterflies with different mean axial stress and shear strain values. The horizontal loading paths E, F, G, and H have been differentiated through non-proportionality factor for their different mean stress values and loading sequences. 

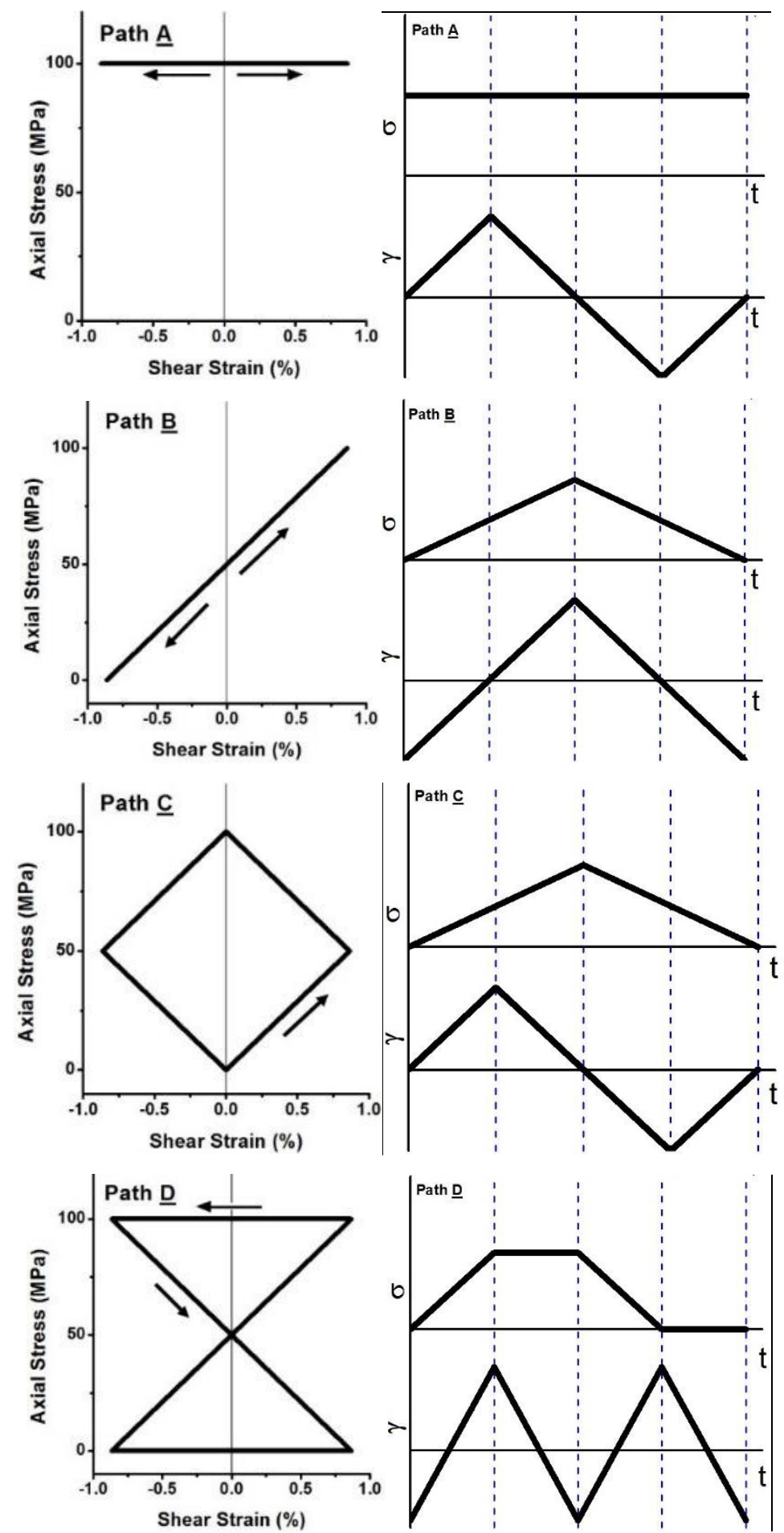

Figure 4.1 Multiaxial loading paths A-H and their loading spectra for 1045 steel samples. 

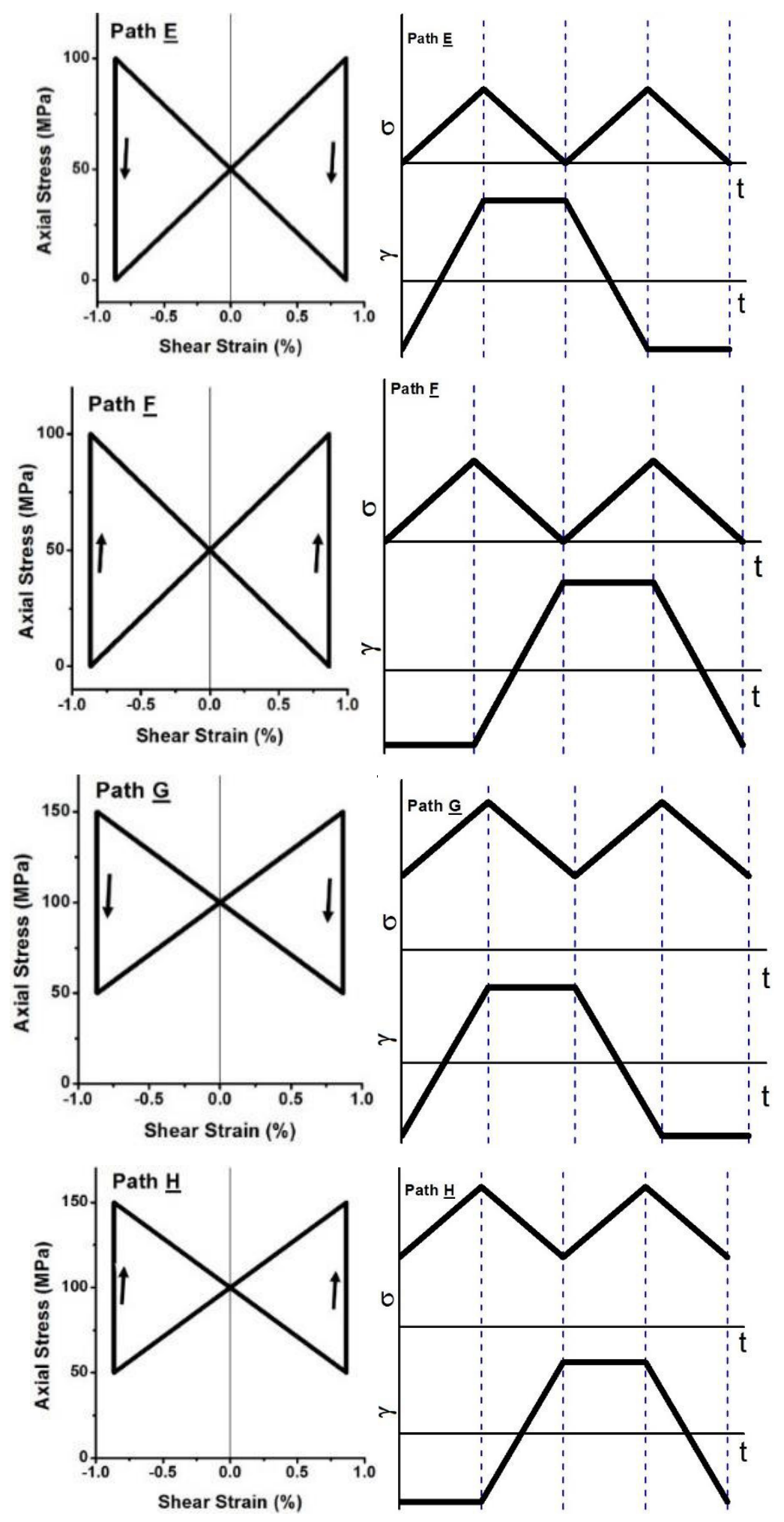

Figure 4.1. Continued 
Table 4.1 Ratcheting experiments for 1045 steel samples under various loading paths [30].

\begin{tabular}{cccccc}
\hline Loading Path & $\boldsymbol{\Delta} \boldsymbol{\gamma} / \mathbf{2}$ & $\boldsymbol{\sigma}_{\mathbf{a}}$ & $\boldsymbol{\sigma}_{\mathbf{m}}$ & $\boldsymbol{\tau}_{\mathbf{a}}$ & $\boldsymbol{\tau}_{\mathbf{m}}$ \\
\hline $\mathbf{A}$ & $0.866 \%$ & 0 & 100 & 288 & 0 \\
\hline $\mathbf{B}$ & $0.866 \%$ & 50 & 50 & 275 & 0 \\
\hline $\mathbf{C}$ & $0.866 \%$ & 50 & 50 & 274 & 0 \\
\hline $\mathbf{D}$ & $0.866 \%$ & 50 & 50 & 280 & 0 \\
\hline $\mathbf{E}$ & $0.866 \%$ & 50 & 50 & 282 & 0 \\
\hline $\mathbf{F}$ & $0.866 \%$ & 50 & 50 & 285 & 0 \\
\hline $\mathbf{G}$ & $0.866 \%$ & 50 & 100 & 290 & 0 \\
\hline $\mathbf{H}$ & $0.866 \%$ & 50 & 100 & 295 & 0 \\
\hline
\end{tabular}

The ratcheting strain values were determined from $\varepsilon_{r}=\frac{1}{2}\left(\varepsilon_{\max }+\varepsilon_{\min }\right)$ where $\varepsilon_{\max }$ and $\varepsilon_{\min }$ are the experimentally obtained maximum and minimum strain values of progressing stress-strain hysteresis loops. Ratcheting strain data of 1045 steel samples of various loading paths A-H were plotted versus multiaxial stress cycles in Fig 4.2. This figure shows how influential is the effect of loading path on the magnitude of ratcheting strain data in steel samples achieved at different stress amplitudes and mean stresses. 


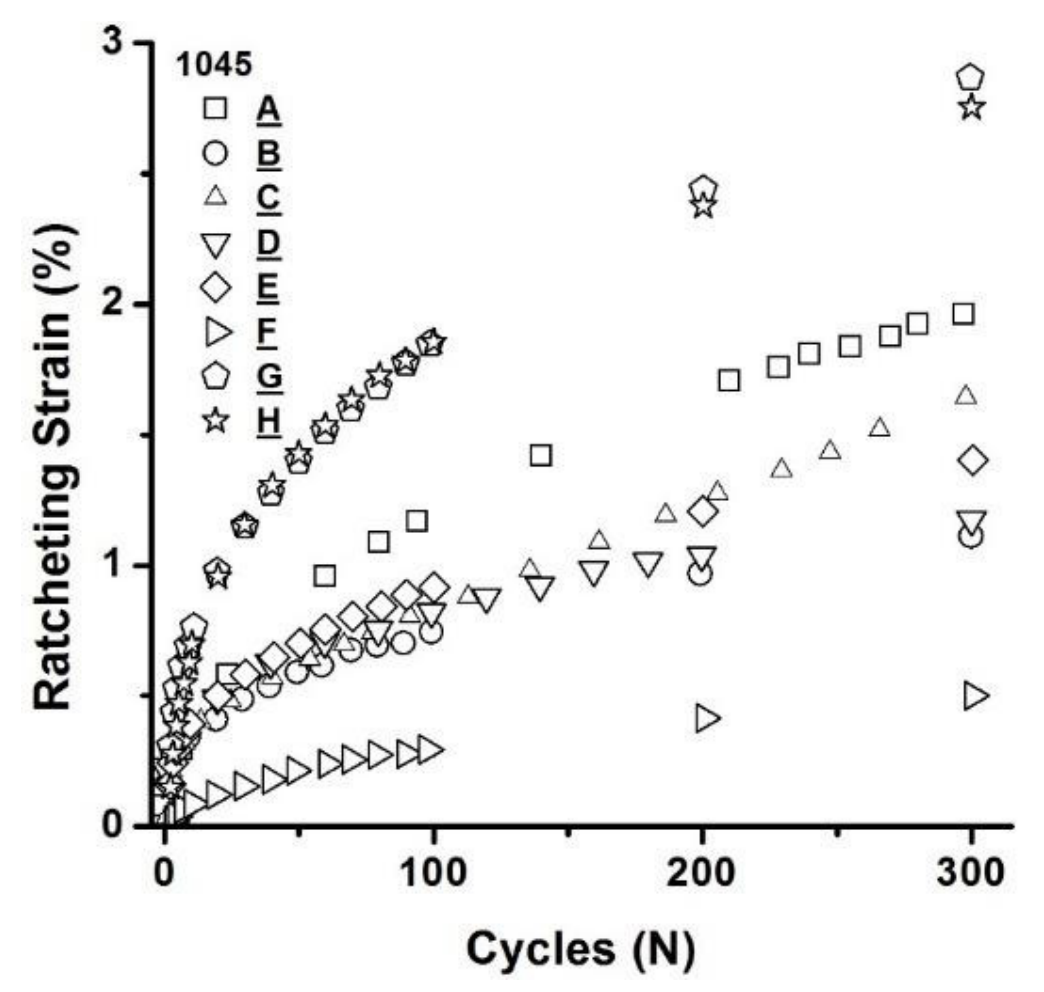

Figure 4.2 Ratcheting strain vs stress cycles for 1045 steel samples under various nonproportional loading paths (A-H) [30].

The material dependent calibrating coefficient $\gamma_{2}$ is determined from experimentally obtained ratcheting strains over uniaxial stress cycles in 1045 steel alloy. Figure 4.3 shows curves of ratcheting calibrated through $\gamma_{2}$ for a 1045 steel sample tested under uniaxial stress cycles of $100 \pm 370$ magnitude. Coefficients of the modified hardening rule for 1045 steel alloy are listed in Table 4.2. 


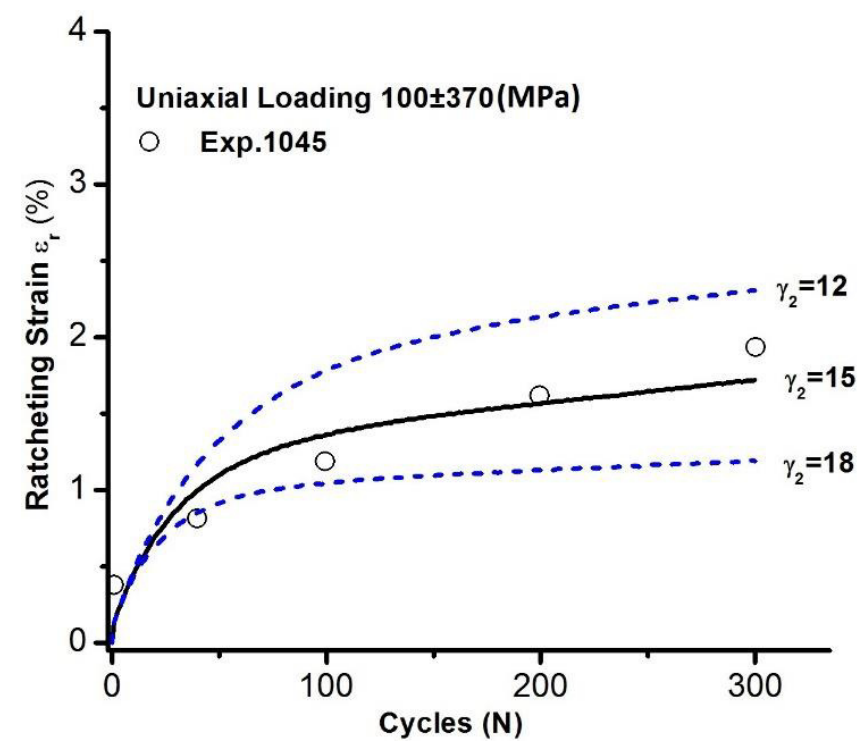

Figure 4.3 Determination of coefficient $\gamma_{2}$ in 1045 steel sample tested under uniaxial condition.

Table 4.2 The coefficients of the modified hardening rule for 1045 steel alloy.

Modified Hardening Rule Parameters $C=150 \mathrm{GPa}, \gamma_{1}=400, \gamma_{2}=15$

\subsubsection{Predicted ratcheting curves of 1045 steel alloy}

The multiaxial ratcheting data of 1045 tubular steel samples were predicted by means of the modified hardening rule. The predicted ratcheting curves were discussed in the presence of modifying parameters in the dynamic recovery term and were compared with experimental data. The modified model similar to other coupled kinematic hardening rules was constructed on the basis of the theory of plasticity elements including Hooke's law, yield criterion, and flow rule. The experimental and the predicted ratcheting strain values based on the modified model (Eq. (3.8)) for 1045 tubular specimen tested under various multiaxial loading paths A-H are presented in figure 4.4. In this figure, ratcheting strains progress in the direction of axial mean stress with a decay in ratcheting rate over multiaxial stress cycles.

Figure 4.4 presents the evolution of the modified hardening rule (Eqn (3.8)) for ratcheting assessment of 1045 steel samples for cases at which (i) the accumulated plastic strain increment 
$d p$ is replaced with $\left\langle d \bar{\varepsilon}_{p} \cdot \bar{a} /|\bar{a}|\right\rangle$ in the dynamic recovery term of equation (3.8a), (ii) the term $\langle\bar{n} \cdot \bar{a} /|\bar{a}|\rangle^{1 / 2}$ in equation (3.8a) is absent, (iii) the term $(2-\bar{n} \cdot \bar{a} /|\bar{a}|)$ in equation (3.8b) is absent, and (iv) the modified hardening rule holding all terms of equation (3.8).
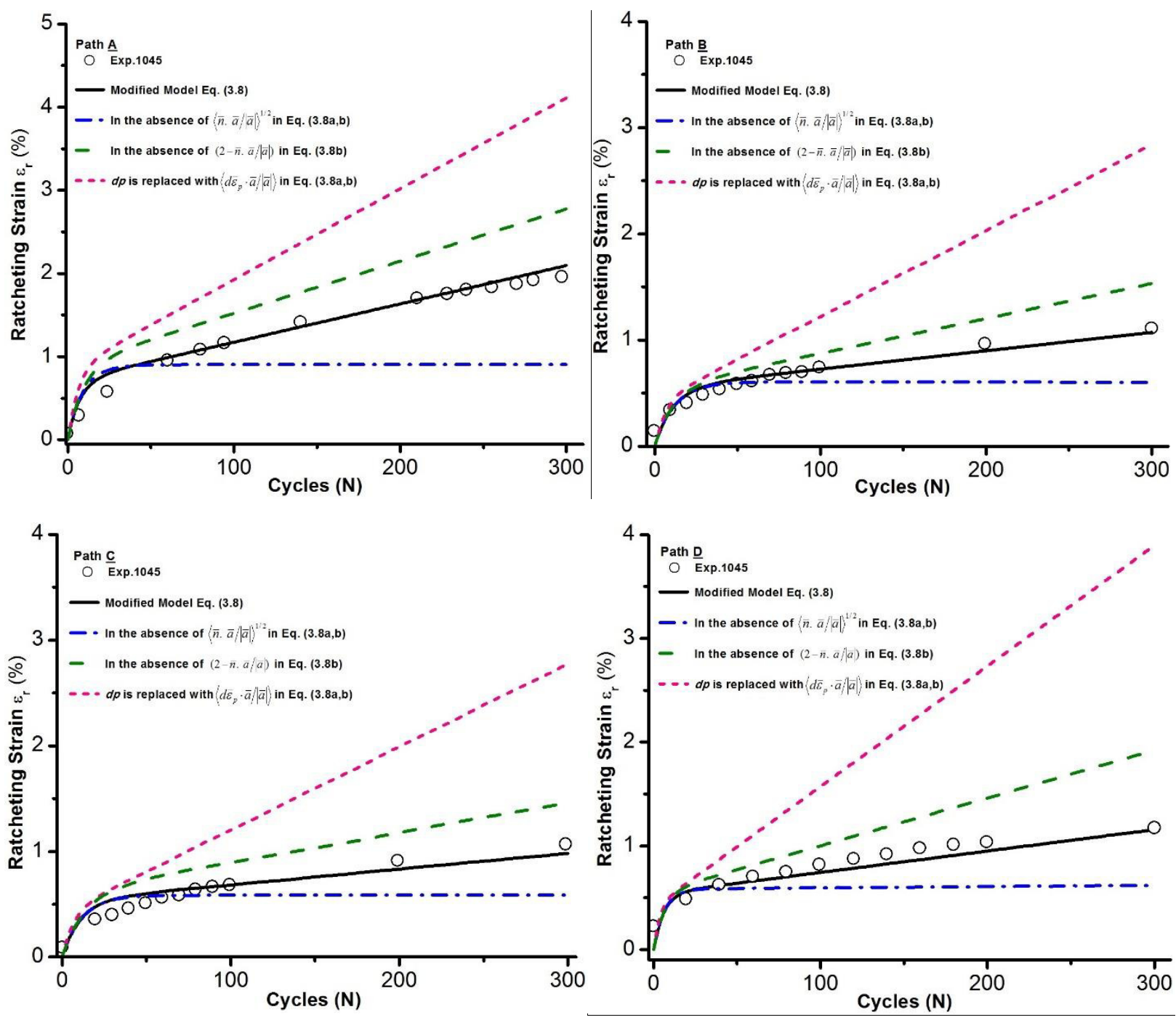

Figure 4.4 Experimental data [30] and predicted ratcheting strain values of 1045 steel samples based on the modified model under various loading conditions (A-H). 

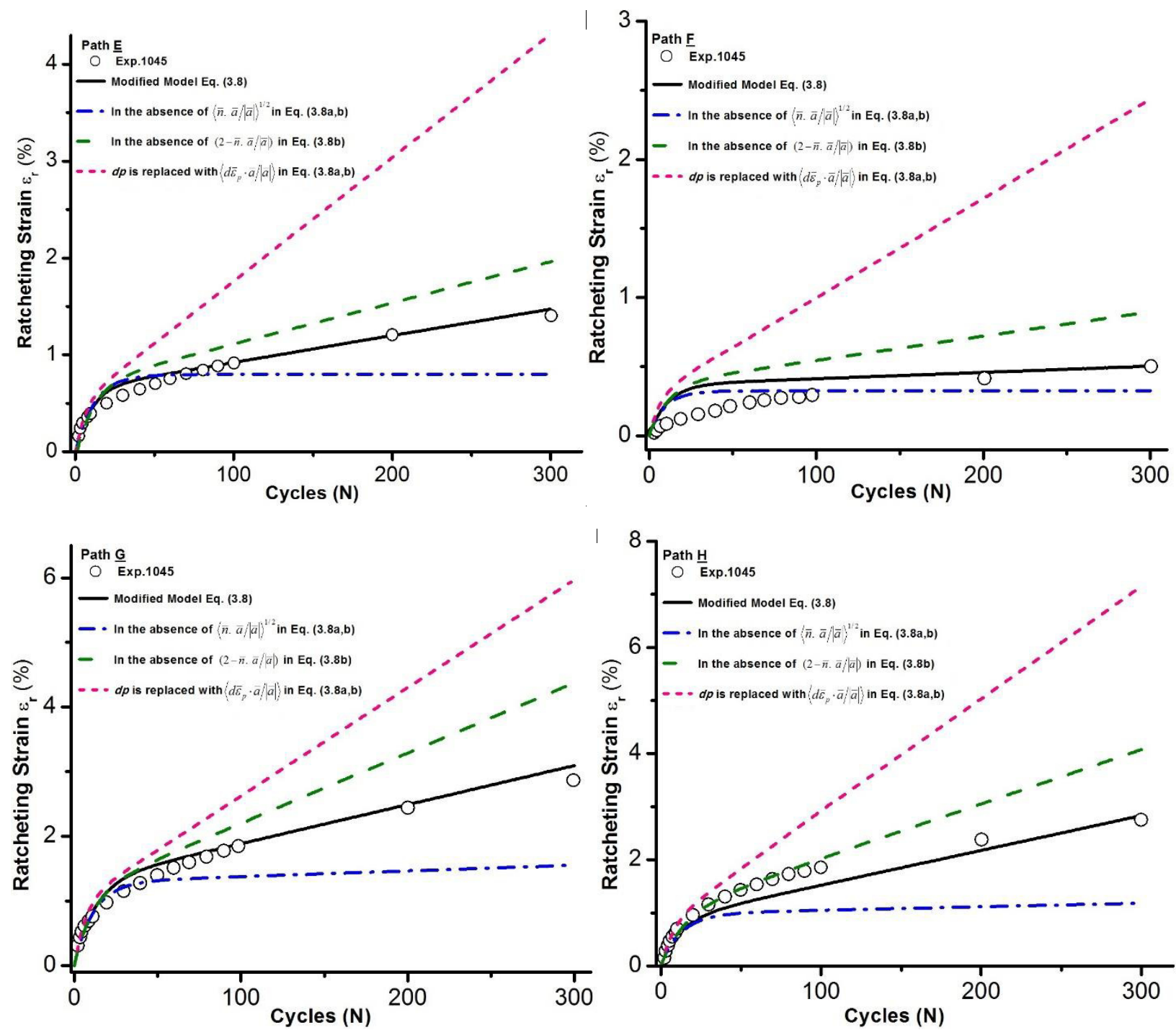

Figure 4.4. Continued.

Experimental ratcheting data in this figure consistently agree with those of predicted curves based on the modified hardening rule for multiaxial loading paths A, B, C, D, and E. The predicted ratcheting curve for the path $\mathrm{F}$ however slightly overestimates the ratcheting data. The modified model closely predicts the trend of experimental ratcheting data over stages I and II under loading path G. The modified model slightly underestimates the ratcheting strain of 1045 samples under loading path $\mathrm{H}$. The overestimation and underestimation in histories $\mathrm{F}$ and $\mathrm{H}$ have been also reported for the predicted ratcheting curves based on the Abdel Karim-Ohno and Chen models in ref. [30]. The close agreements of the modified models with experimental data are attributed to the 
inclusion of material dependent coefficients $\mathrm{C}, \gamma_{1}$, and $\gamma_{2}$. The non-proportionality has been introduced in the dynamic recovery term through terms $\left\langle d \bar{\varepsilon}_{p} \cdot \bar{a} /|\bar{a}|\right\rangle$ and $(\bar{n} \cdot \bar{a} /|\bar{a}|)$. The modified model (Eqn (3.8)) holding the calibrating coefficient $\gamma_{2}$, terms $\left\langle d \bar{\varepsilon}_{p} \cdot \bar{a} /|\bar{a}|\right\rangle$ and $(\bar{n} . \bar{a} /|\bar{a}|)$ offered a reasonable agreement with multiaxial ratcheting data obtained experimentally. As $d p$ in dynamic recovery term of the $\mathrm{A}-\mathrm{V}$ hardening rule is replaced with $\left\langle d \bar{\varepsilon}_{p} \cdot \bar{a} /|\bar{a}|\right\rangle$, the capability of the hardening rule has been further improved to assess multiaxial ratcheting. The function in the MaCaulay brackets reduces to $d p$ under uniaxial loading condition. This function results in slower accumulation of ratcheting strain rate. Since $d \bar{\varepsilon}_{p}$ and $\bar{a} /|\bar{a}|$ possess different directions under multiaxial stress cycles, their projection presented by $\left\langle d \bar{\varepsilon}_{p} \cdot \bar{a} /|\bar{a}|\right\rangle$ is rather smaller and more controllable. The function in the MaCaulay brackets in the dynamic recovery term shifted down the overall magnitude of predicted ratcheting curve as substituted with the accumulated plastic strain increment $d p$. Figure 4.4 shows how influential this substitution in the dynamic recovery term is. In the framework of the proposed model, term $(\bar{n} \cdot \bar{a} /|\bar{a}|)$ accounts for non-proportionality under multiaxial loading conditions. The normal vector $\bar{n}$ to the yield surface, and the unity vector of backstress $\bar{a} /|\bar{a}|$ have different directions under multiaxial loading, and consequently the projection presented by $(\bar{n} . \bar{a} /|\bar{a}|)$ produces values between -1 and 1 and becomes 1 under uniaxial case. Term $(2-\bar{n} \cdot \bar{a} /|\bar{a}|)$ becomes equal to unity under uniaxial loading case and takes the values between 1 and 3 under multiaxial loading conditions. The product of this term and calibrating coefficient $\gamma_{2}$ results in a slower rate of ratcheting strain accumulated over stress cycles. This is evident from figure 4.4 as the predicted ratcheting solid curve based on the modified model (Eqn (3.8b)) holding $(2-\bar{n} \cdot \bar{a} /|\bar{a}|)$ term in the dynamic recovery and the green dashed curve predicted in the absence of $(2-\bar{n} . \bar{a} /|\bar{a}|)$ term are compared. In the absence of this term embracing nonproportionality term $(\bar{n} \cdot \bar{a} /|\bar{a}|)$ in this predicted curve, ratcheting values show overprediction. More overprediction of ratcheting (pink dashed curve) is resulted in through the replacement of 
$\left\langle d \bar{\varepsilon}_{p} \cdot \bar{a} /|\bar{a}|\right\rangle$ with the accumulated plastic strain increment $d p$ in the modified model (see figure 4.4). This predicted curve shows how influential the effect of $\left\langle d \bar{\varepsilon}_{p} \cdot \bar{a} /|\bar{a}|\right\rangle$ is in the prediction of ratcheting values. The blue dash-dot curve in this figure represents the modified rule in the absence of $\langle\bar{n} . \bar{a} /|\bar{a}|\rangle^{1 / 2}$ in the dynamic recovery term resulting in plastic shakedown over stage II. Under multiaxial loading histories, this term takes positive values smaller than 1 and prevents this model from premature plastic shakedown while it reduces to unity under uniaxial loading conditions.

\subsection{Multiaxial ratcheting assessment of 1045 and $1 \mathrm{Cr} 18 \mathrm{Ni}$ Ti steel alloys based on the O-W, J-S, McDowell and A-V modified hardening rules}

Ratcheting response of 1045 and $1 \mathrm{Cr} 18 \mathrm{Ni9Ti}$ tubular steel samples are predicted using nonlinear kinematic hardening rules of Ohno-Wang (O-W), Jiang-Sehitoglu (J-S), McDowell and newly modified model based on hardening rule of Ahmadzadeh-Varvani (A-V) under various multiaxial loading histories. The $\mathrm{O}-\mathrm{W}$ hardening rule was developed based on the critical state of dynamic recovery of backstress. The total backstress was determined by the superimposition of $M$ independent backstress components first suggested by Chaboche holding several coefficients. The $\mathrm{J}-\mathrm{S}$ and McDowell hardening rules further developed the $\mathrm{O}-\mathrm{W}$ rule to include the effects of stressdependency and non-proportionality in ratcheting assessment of materials. The modified

hardening rule with less complexity holds terms $\left\langle d \bar{\varepsilon}_{p} \cdot \bar{a} /|\bar{a}|\right\rangle,(2-\bar{n} \cdot \bar{a} /|\bar{a}|)$ and $\langle\bar{n} \cdot \bar{a} /|\bar{a}|\rangle^{1 / 2}$ to encounter the non-proportionality effects in its dynamic recovery term dictating the evolution of the yield surface on the deviatoric stress space to accumulate the ratcheting strain with the gradual decreasing rate. 


\subsubsection{5 and $1 \mathrm{Cr} 18 \mathrm{Ni}$ Ti steel samples, testing and multiaxial ratcheting data}

Ratcheting experimental data of 1045 steel [30] and 1Cr18Ni9Ti stainless steel [29] samples were taken to evaluate the capability of the $\mathrm{O}-\mathrm{W}, \mathrm{J}-\mathrm{S}$, McDowell and modified A-V hardening rules in ratcheting assessment under various multiaxial loading paths. Ratcheting tests on 1045 and 1Cr18Ni9Ti steel samples under multiaxial loading conditions were conducted at both stresscontrolled and strain-controlled cycles which were responsible for ratcheting of steel samples. The different multiaxial and loading paths conducted on samples of 1045 are presented in Fig. 4.5 (Paths A-H). The experimental details of 1045 steel samples were presented in section 4.1.1.

The samples of $1 \mathrm{Cr} 18 \mathrm{Ni}$ Ti consisted inside and outside diameters of 18 and $22 \mathrm{~mm}$ in the gauge section and were subjected to multiaxial with a frequency of $0.5 \mathrm{~Hz}$ at room temperature. An MTS axial-torsional extensometer mounted on the outside of the gauge section of $1 \mathrm{Cr} 18 \mathrm{Ni}$ Ti steel

sample was used to measure the strain over cycles. The axial-torsional loading histories of 1Cr18Ni9Ti samples are shown in Fig. 4.5 (Paths I-K). 

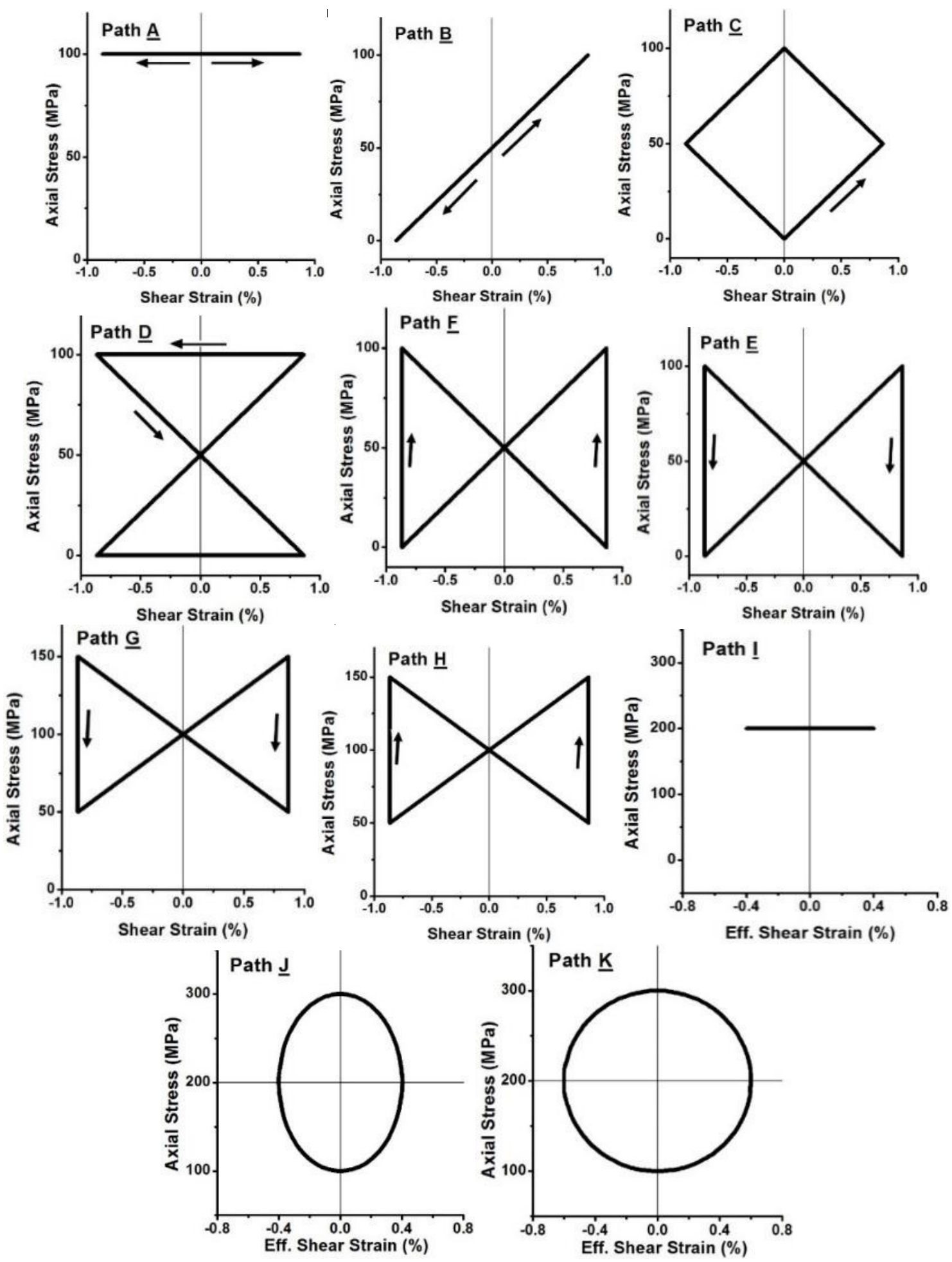

Figure 4.5 Multiaxial loading paths and their loading spectra for 1045 (A-H) and 1Cr18Ni9Ti (IK) steel samples. 
Table 4.3 presents ratcheting tests and loading conditions for tubular $1 \mathrm{Cr} 18 \mathrm{Ni}$ Ti and 1045 steel samples. The experiments on thin-walled tubular samples of $1 \mathrm{Cr} 18 \mathrm{Ni}$ Ti stainless steel were carried out [29] under axial stress-shear strain cycles.

Table 4.3 Ratcheting experiments for 1045 and 1Cr18Ni9Ti steel samples under various loading

\begin{tabular}{|c|c|c|c|c|c|c|}
\hline Material & Loading Path & $\Delta \gamma / 2$ & $\sigma_{\mathbf{a}}$ & $\sigma_{m}$ & $\tau_{\mathbf{a}}$ & $\tau_{m}$ \\
\hline \multirow{8}{*}{1045} & $\mathbf{A}$ & $0.866 \%$ & 0 & 100 & 288 & 0 \\
\hline & $\overline{\mathbf{B}}$ & $0.866 \%$ & 50 & 50 & 275 & 0 \\
\hline & $\bar{C}$ & $0.866 \%$ & 50 & 50 & 274 & 0 \\
\hline & $\bar{D}$ & $0.866 \%$ & 50 & 50 & 280 & $\overline{0}$ \\
\hline & $\bar{E}$ & $0.866 \%$ & 50 & 50 & 282 & 0 \\
\hline & $\mathbf{F}$ & $0.866 \%$ & 50 & 50 & 285 & 0 \\
\hline & $\mathbf{G}$ & $0.866 \%$ & 50 & 100 & 290 & 0 \\
\hline & $\bar{H}$ & $0.866 \%$ & 50 & 100 & 295 & 0 \\
\hline \multirow{3}{*}{ 1Cr18Ni9Ti } & I & $0.4 \%$ & 0 & 200 & 278 & 0 \\
\hline & $\mathbf{J}$ & $0.4 \%$ & 100 & 200 & 302 & 0 \\
\hline & $\bar{K}$ & $0.6 \%$ & 100 & 200 & 327 & 0 \\
\hline
\end{tabular}

The stainless steel samples of 1Cr18Ni9Ti were tested with loading paths I, J and K. Path I is similar to path A and consists of fully reversed shear strain cycle with a constant axial stress, while paths $\mathrm{J}$ and $\mathrm{K}$ represent 90 out-of-phase of sinusoidal shear strain and axial stress spectra which result in elliptical and circular paths respectively possessing $0.4 \%$ and $0.6 \%$ shear strain amplitudes. 


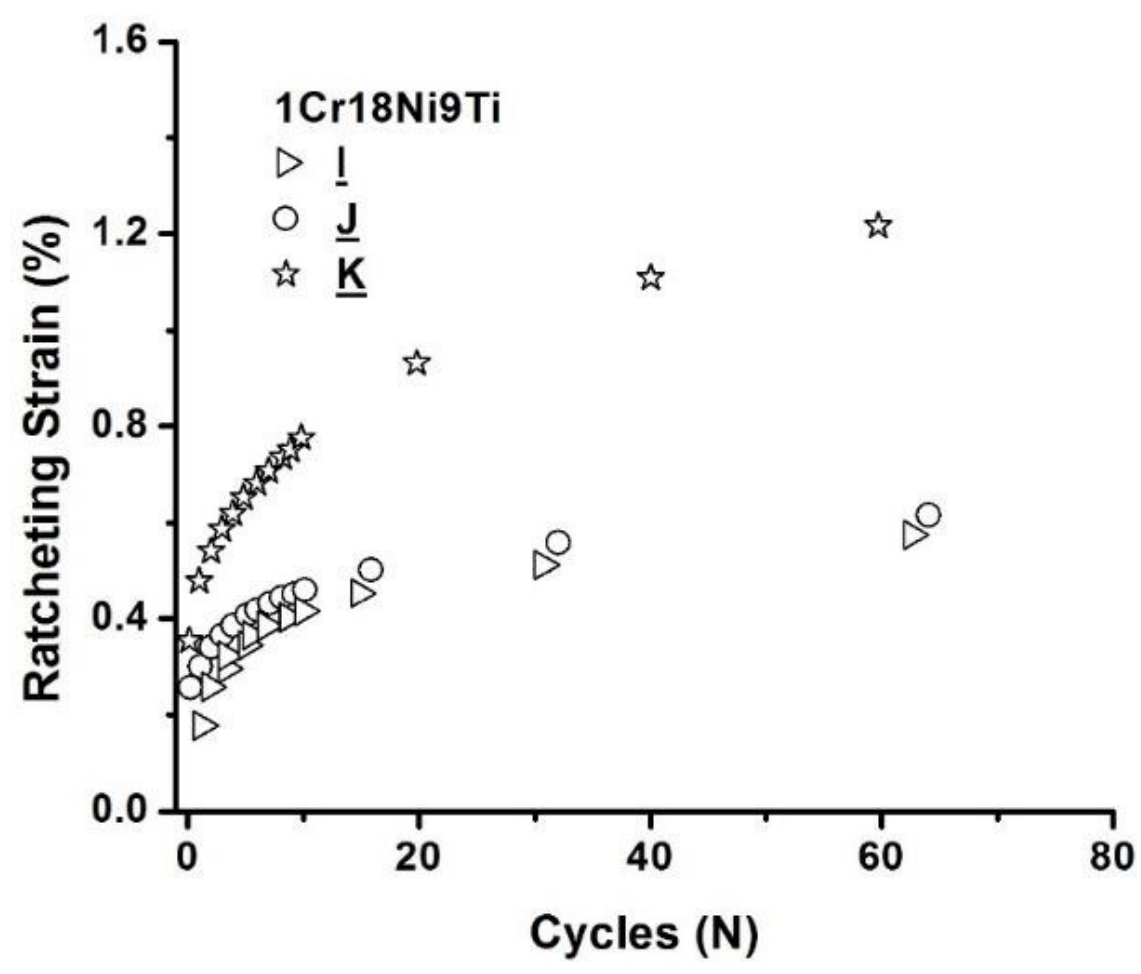

Figure 4.6 Ratcheting strain vs stress cycles for $1 \mathrm{Cr} 18 \mathrm{Ni}$ Ti (I-K) steel samples under various non-proportional loading paths [29].

The coefficients of the $\mathrm{O}-\mathrm{W}, \mathrm{J}-\mathrm{S}, \mathrm{McD}$ owell hardening rules and the modified model employed in the current study are listed in Table 4.4.

Ratcheting strain data of 1045 and $1 \mathrm{Cr} 18 \mathrm{Ni} 9 \mathrm{Ti}$ steel samples of various loading paths were plotted versus multiaxial stress cycles in figures 4.2 and 4.6, respectively. This figures shows how influential the effect of loading path is on the magnitude of ratcheting strain data in steel samples achieved at different loading paths, stress amplitudes and mean stresses. 
Table 4.4 The coefficients of the hardening rules to assess ratcheting response of 1045 and 1Cr18Ni9Ti steel samples.

\begin{tabular}{|c|c|c|}
\hline Material & Model & Hardening Rule Parameter \\
\hline \multirow{4}{*}{1045} & $\begin{array}{c}\text { Modified } \\
\text { Model }\end{array}$ & $C=150 \mathrm{GPa}, \gamma_{1}=400, \gamma_{2}=16$ \\
\hline & $\begin{array}{c}O-W \\
\text { Model }\end{array}$ & $\begin{array}{c}\gamma_{1}=2500, \gamma_{2}=1250, \gamma_{3}=666.7, \gamma_{4}=500, \gamma_{5}=333.3, \gamma_{6}=200, \gamma_{7}=125, \gamma_{8}=83.3, \\
r_{1}=88, r_{2}=42, r_{3}=30.4, r_{4}=21.3, r_{5}=28.3, r_{6}=31, r_{7}=27.8, r_{8}=76.4 \\
m_{i}=3.7\end{array}$ \\
\hline & $\begin{array}{c}J-S \\
\text { Model }\end{array}$ & $\begin{array}{c}\gamma_{1}=2500, \gamma_{2}=1250, \gamma_{3}=666.7, \gamma_{4}=500, \gamma_{5}=333.3, \gamma_{6}=200, \gamma_{7}=125, \gamma_{8}=83.3, \\
r_{1}=88, r_{2}=42, r_{3}=30.4, r_{4}=21.3, r_{5}=28.3, r_{6}=31, r_{7}=27.8, r_{8}=76.4 \\
m_{0 i}=3.7\end{array}$ \\
\hline & $\begin{array}{c}\text { McDowell } \\
\text { Model }\end{array}$ & $\begin{array}{c}\gamma_{1}=2500, \gamma_{2}=1250, \gamma_{3}=666.7, \gamma_{4}=500, \gamma_{5}=333.3, \gamma_{6}=200, \gamma_{7}=125, \gamma_{8}=83.3, \\
r_{1}=88, r_{2}=42, r_{3}=30.4, r_{4}=21.3, r_{5}=28.3, r_{6}=31, r_{7}=27.8, r_{8}=76.4 \\
A_{i}=3.7, B_{i}=0.5\end{array}$ \\
\hline \multirow{4}{*}{$1 \mathrm{Cr} 18 \mathrm{Ni9Ti}$} & $\begin{array}{c}\text { Modified } \\
\text { Model }\end{array}$ & $C=200 \mathrm{GPa}, \gamma_{1}=370, \gamma_{2}=54$ \\
\hline & $\begin{array}{c}O-W \\
\text { Model }\end{array}$ & $\begin{array}{c}\gamma_{1}=4800, \gamma_{2}=2400, \gamma_{3}=1200, \gamma_{4}=600, \gamma_{5}=300, \gamma_{6}=150, \gamma_{7}=75, \gamma_{8}=37.5 \\
r_{1}=10, r_{2}=65, r_{3}=63, r_{4}=41, r_{5}=80, r_{6}=70, r_{7}=16, r_{8}=2 \\
m_{i}=30\end{array}$ \\
\hline & $\begin{array}{c}J-S \\
\text { Model }\end{array}$ & $\begin{array}{c}\gamma_{1}=4800, \gamma_{2}=2400, \gamma_{3}=1200, \gamma_{4}=600, \gamma_{5}=300, \gamma_{6}=150, \gamma_{7}=75, \gamma_{8}=37.5 \\
r_{1}=10, r_{2}=65, r_{3}=63, r_{4}=41, r_{5}=80, r_{6}=70, r_{7}=16, r_{8}=2 \\
m_{0 i}=30\end{array}$ \\
\hline & $\begin{array}{l}\text { McDowell } \\
\text { Model }\end{array}$ & $\begin{array}{c}\gamma_{1}=4800, \gamma_{2}=2400, \gamma_{3}=1200, \gamma_{4}=600, \gamma_{5}=300, \gamma_{6}=150, \gamma_{7}=75, \gamma_{8}=37.5 \\
r_{1}=10, r_{2}=65, r_{3}=63, r_{4}=41, r_{5}=80, r_{6}=70, r_{7}=16, r_{8}=2 \\
A_{i}=30, B_{i}=0.5\end{array}$ \\
\hline
\end{tabular}

\subsubsection{Predicted ratcheting of 1045 and 1 Cr18Ni9Ti steel alloys by $\mathrm{O}-\mathrm{W}, \mathrm{J}-\mathrm{S}$, McDowell and A-V modified hardening rules}

The multiaxial ratcheting response of 1045 and $1 \mathrm{Cr} 18 \mathrm{Ni}$ Ti steel samples were predicted by means of the $\mathrm{O}-\mathrm{W}, \mathrm{J}-\mathrm{S}, \mathrm{McD}$ owell and modified A-V kinematic hardening rules. The experimental and the predicted ratcheting strain values based on the modified model (Eq. (3.8)), and the hardening rules of O-W (Eq. (2.8)), J-S (Eq. (2.11-2.12)), McDowell (Eq. (2.10)) for 1045 and $1 \mathrm{Cr} 18 \mathrm{Ni}$ Ti samples tested under various multiaxial loading paths are presented in figures 4.7 and 4.8 respectively. Ratcheting strains progressed in the direction of the axial mean stress with a decay in the accumulated ratcheting rate over multiaxial stress cycles. The capability of the $\mathrm{O}-\mathrm{W}$, 
$\mathrm{J}-\mathrm{S}, \mathrm{McD}$ owell and A-V modified hardening rules in ratcheting assessment of 1045 steel sample under eight different loading paths (A-H) was assessed as the predicted ratcheting curves were compared with those of experimentally reported values in Fig. 4.7. Experimental ratcheting data in this figure show consistently good agreements with those of predicted curves based on the modified hardening rule for multiaxial loading paths A, B, C, D, and E. The predicted ratcheting curve for path $\mathrm{F}$ however slightly overestimates the ratcheting data. The modified model closely predicts the trend of experimental ratcheting data over stages I and II under loading path G. The modified model underestimates the ratcheting strain of 1045 samples under loading path $\mathrm{H}$. The overestimation and underestimation of respectively histories $\mathrm{F}$ and $\mathrm{H}$ have been also reported for the predicted ratcheting curves based on the Abdel Karim -Ohno and Chen models in ref. [30].

The close agreement between the predicted ratcheting curves based on the modified model with experimental data for various loading histories is attributed to the inclusion of nonproportionality through terms $\left\langle d \bar{\varepsilon}_{p} \cdot \bar{a} /|\bar{a}|\right\rangle$ and $(\bar{n} . \bar{a} /|\bar{a}|)$ in the dynamic recovery. The modified hardening rule substitutes $\left\langle d \bar{\varepsilon}_{p} \cdot \bar{a} /|\bar{a}|\right\rangle$ with the accumulated plastic strain increment, $d p$ in the dynamic recovery term of the model. This enables the modified model to follow different directions under multiaxial loading due to components $d \bar{\varepsilon}_{p}$ and $\bar{a} /|\bar{a}|$. Under multiaxial loading condition, the projection presented by the function in the MaCaulay brackets is smaller than $d p$ resulting in a slower evolution in simulation of ratcheting strain under multiaxial loading conditions. 

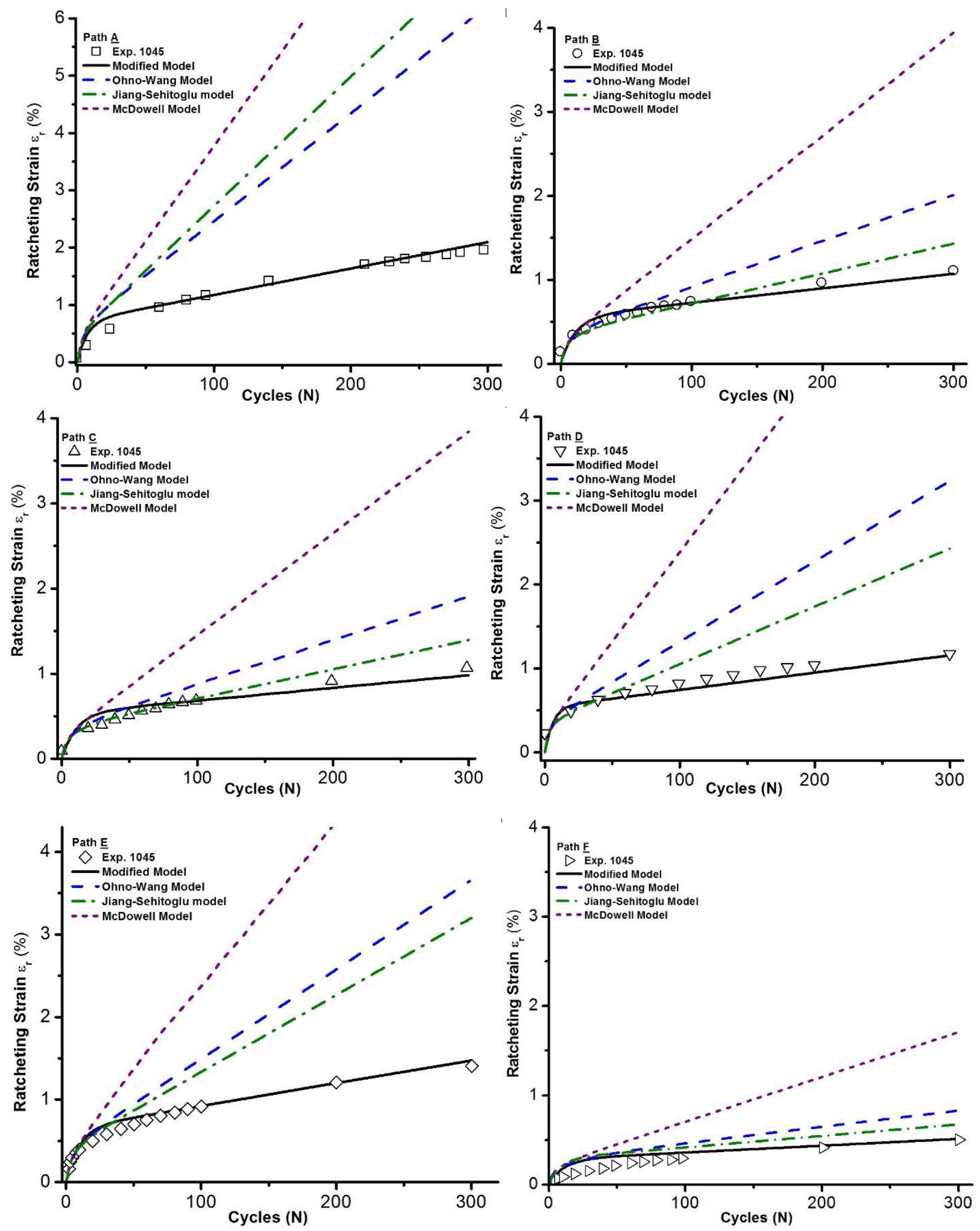

Figure 4.7 Experimental data [30] and predicted ratcheting strain values of 1045 steel samples based on the Ohno-Wang (O-W), Jiang-Sehitoglu (J-S), McDowell and A-V modified hardening rules under various loading conditions $(\mathrm{A}-\mathrm{H})$. 

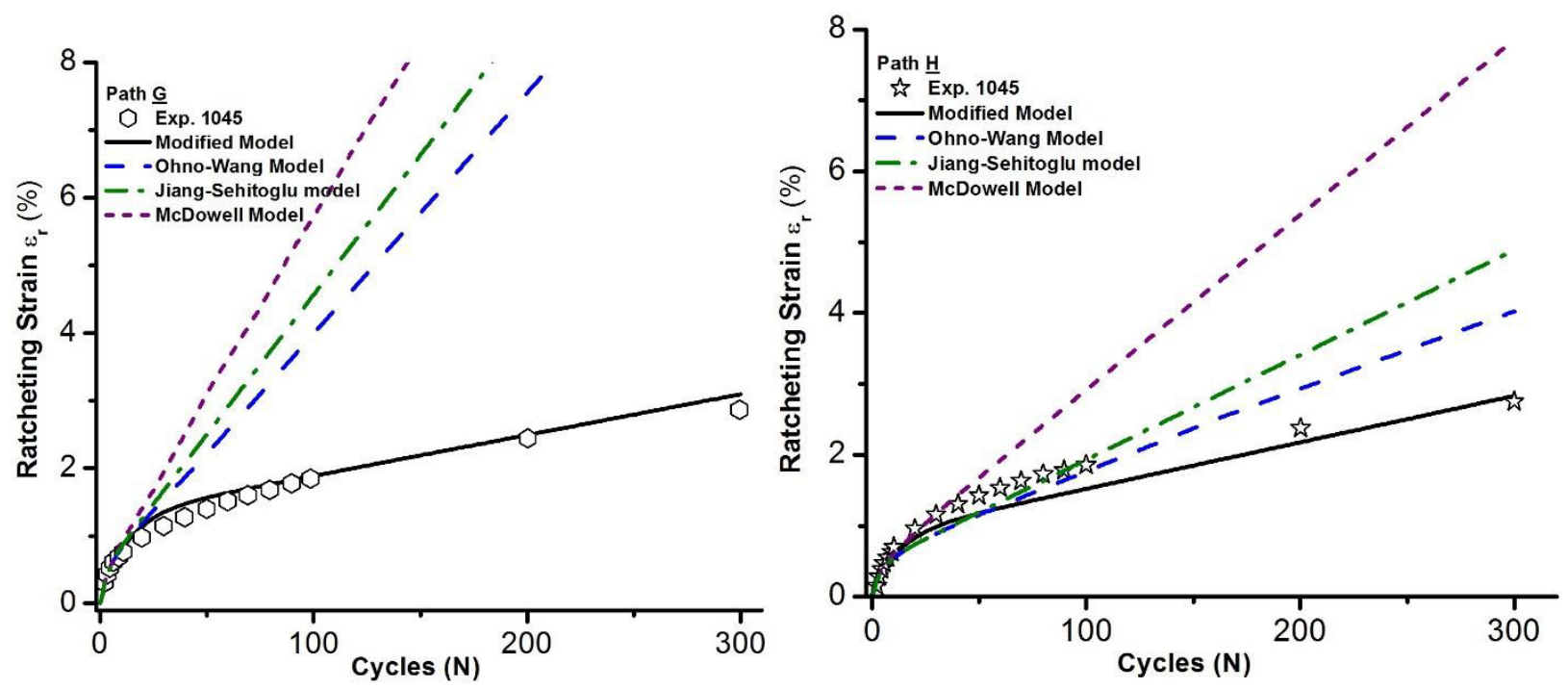

Figure 4.7. Continued

As presented in figures 4.7 and 4.8 , it is quite evident that the $\mathrm{O}-\mathrm{W}$ model with $m_{i}=3.7$ overestimated ratcheting response for various multiaxial loading paths. Larger values of $m_{i}$ in equation (2.8) can decrease the rate of accumulated ratcheting over multiaxial stress cycles. However, this adjustment can affect the simulations response of uniaxial ratcheting. Chen and Jiao [29] have reported that the O-W model overestimates the ratcheting response of cyclically hardened materials under multiaxial loading. This overestimation was earlier attributed to the lack of parameters reflecting non-proportionality to control the evolution of ratcheting over multiaxial stress cycles [36].

The J-S hardening rule has also shown a persistent overestimation in various loading conditions of steel samples in figures 4.7 and 4.8. Based on the definition of exponent $m_{i}$ in equation (2.12), different directions possessed by terms $\bar{a}_{i} /\left|\bar{a}_{i}\right|$ and $\bar{n}$ over multiaxial stress cycles resulted in function $\bar{n} \cdot \bar{a}_{i} /\left|\bar{a}_{i}\right|$ to fall between -1 and 1 and consequently exponent $m_{i}$ in the J-S hardening rule becomes larger than $m_{0 i}=3.7$. The higher value of $m_{i}$ diminishes the rate of accumulated ratcheting as compared with that of the $\mathrm{O}-\mathrm{W}$ rule. The interactive parameter $d p$ however in the J-S model tends to expedite the rate of ratcheting contrarily. The predicted ratcheting curve based on the J-S rule showed less overestimation than the O-W model for loading 
paths B, C, D, E, and F. The O-W model however showed better prediction for loading paths A, $\mathrm{H}$, and $\mathrm{G}$.

Term $\left\langle d \bar{\varepsilon}_{p} \cdot \bar{a}_{i} /\left|\bar{a}_{i}\right|\right\rangle$ in the $\mathrm{O}-\mathrm{W}$ model resulted in lower ratcheting strain values under multiaxial stress cycles as compared with term $d p$ in the dynamic recovery term of the $\mathrm{J}-\mathrm{S}$ model. Under loading paths $\mathrm{A}, \mathrm{H}$, and $\mathrm{G}$ with larger axial mean stress of $\sigma_{m}=100 \mathrm{MPa}$ and axial stress ratio of $R>1 / 3$, the smaller rate of accumulated ratcheting strain introduced by $\left\langle d \bar{\varepsilon}_{p} \cdot \bar{a}_{i} /\left|\bar{a}_{i}\right|\right\rangle$ in the $\mathrm{O}-\mathrm{W}$ model is more pronounced. A choice of larger value of exponent $m_{i}$ introduced through equation (2.12) overcame the lower rate of accumulated ratcheting strain through the O-W rule. Loading paths B, C, D, E, and F presented in figure 4.7 show that the exponent $m_{i}$ introduced by the J-S model under multiaxial loading histories, is more influential than term $\left\langle d \bar{\varepsilon}_{p} \cdot \bar{a}_{i} /\left|\bar{a}_{i}\right|\right\rangle$ in the O-W model to decrease the accumulated rate of ratcheting strain. Chen et al. [30] reported the predicted ratcheting curves of medium carbon steel samples tested under multiaxial loading conditions based on the $\mathrm{O}-\mathrm{W}$ and $\mathrm{J}-\mathrm{S}$ hardening rules. In their ratcheting assessment, the dominancy of the mean stress level, stress ratio over exponent $m_{i}$ on the hardening rules was not discussed. Upon replacing $d p$ in the dynamic recovery term of equation (2.11) with term $\left\langle d \bar{\varepsilon}_{p} \cdot \bar{a}_{i} /\left|\bar{a}_{i}\right|\right\rangle$, the J-S hardening rule transforms to the O-W model resulting in a remarkable reduction in ratcheting strain of steel samples undergoing multiaxial stress cycles.

The McDowell model largely overestimated the ratcheting response of steel samples under various loading paths A-H. Predicted ratcheting strain values based on the McDowell model stayed higher than the $\mathrm{O}-\mathrm{W}$ and J-S models. Under uniaxial loading condition the McDowell model reduced to the $\mathrm{O}-\mathrm{W}$ rule. The exponent $m_{i}$ defined based on equation (2.10) enabled the hardening rule with term $\left\langle\bar{n} \cdot \bar{a}_{i} /\left|\bar{a}_{i}\right|\right\rangle$ of the dynamic recovery possessed values between 0 and 1 under multiaxial loading conditions. At $B_{i}>0$ exponent $m_{i}$ computed by this equation stayed smaller than $m_{i}$ determined for the $\mathrm{O}-\mathrm{W}$ model. Consequently McDowell model resulted in larger ratcheting strain values as compared with the $\mathrm{O}-\mathrm{W}$ model. The higher level of non-proportionality, 
larger relative directions of $\bar{a}_{i} /\left|\bar{a}_{i}\right|$ and $\bar{n}$, the lower level of nonlinearity in the dynamic recovery term in the McDowell model resulted in higher rate of ratcheting strain accumulation. An increase in the $B_{i}$ caused more overestimation of the McDowell model. At $B_{i}=0$ the McDowell model reduced to the $\mathrm{O}-\mathrm{W}$ model (Eq. 2.8). This value for medium carbon steel was taken as $B_{i}=1.0$ [30] and for steel samples in the present study $B_{i}=0.5$.

Figure 4.8 presents the predicted ratcheting curves of $\mathrm{Cr} 18 \mathrm{Ni} 9 \mathrm{Ti}$ steel samples tested under multiaxial load histories I-K. The ratcheting curves were predicted based on the O-W, J-S, and McDowell hardening rules considering eight backstress components $M=8$. These predicted ratcheting curves largely overestimated the experimental data.

The predicted ratcheting curves in figure 4.8 based on the modified hardening rule were found in good agreement with those of experimentally reported for histories I, J, and K at which the axial mean stress is $\sigma_{m}=200 \mathrm{MPa}$ with a minimum axial stress ratio of $R=1 / 3$. The predicted ratcheting curve based on the J-S model in figure 4.8 positioned slightly higher than those of the O-W model. Term $\left\langle d \bar{\varepsilon}_{p} \cdot \bar{a}_{i} /\left|\bar{a}_{i}\right|\right\rangle$ in the dynamic recovery of the O-W model dropped the accumulated ratcheting rate for load histories I-K. The lower rate of accumulated ratcheting strain caused by the $\mathrm{O}-\mathrm{W}$ rule overcame the choice of larger value of exponent $m_{i}$ introduced through equations (2.11) and (2.12). With increase in values of $m_{i}, m_{0 i}, A_{i}$ the capability of the O-W, J-S, and McDowell hardening rules in ratcheting prediction of $1 \mathrm{Cr} 18 \mathrm{Ni} 9 \mathrm{Ti}$ steel samples was improved. To predict multiaxial ratcheting based on these hardening rules, the constant values were increased up to 30 to achieve the optimal ratcheting results. This however considerably increased the CPU running time for these models as compared to the modified model with twice shorter CPU time capability in ratcheting assessment of steel samples. 

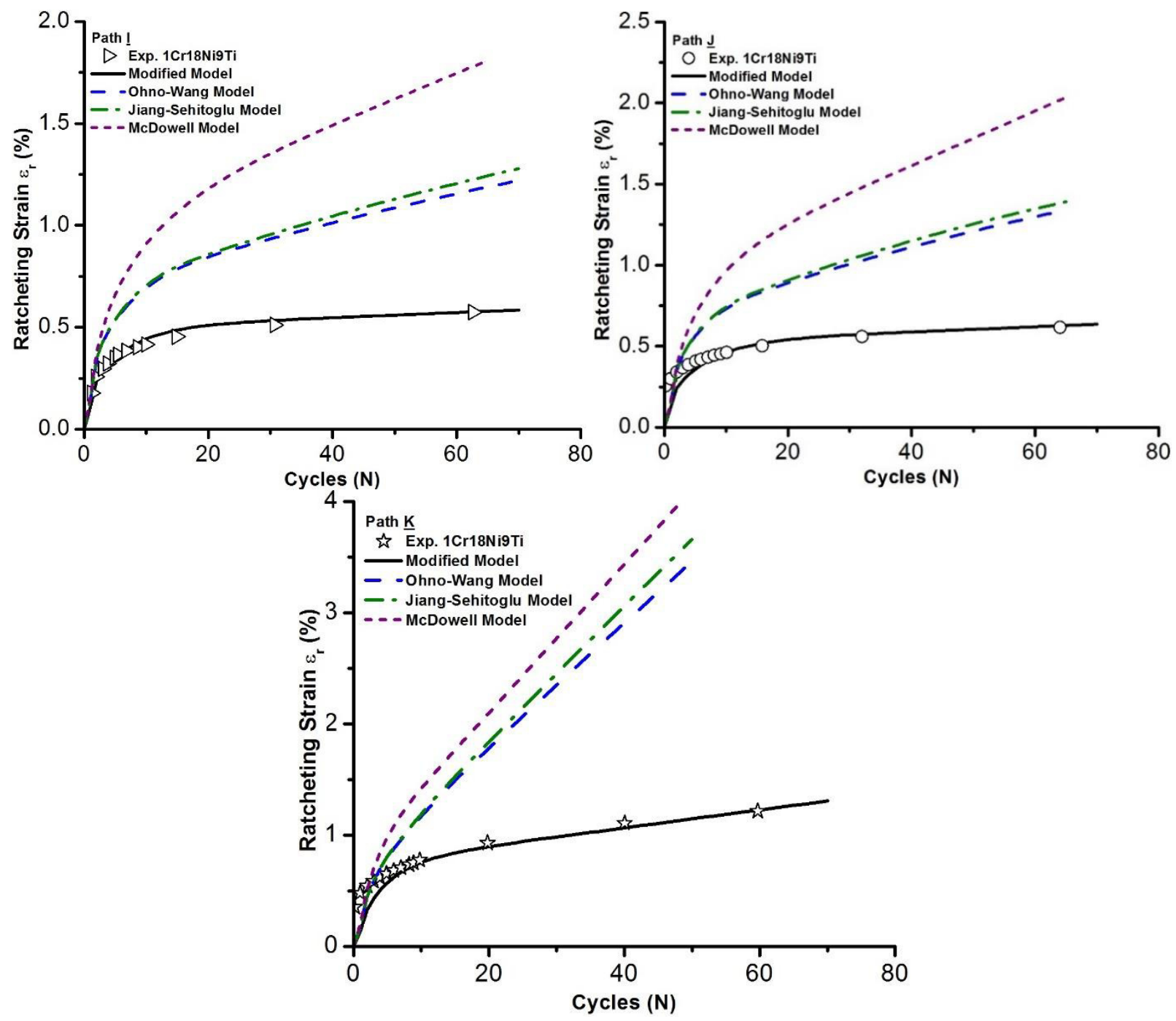

Figure 4.8 Experimental data [29] and predicted ratcheting strain values of 1Cr18Ni9Ti steel samples based on the Ohno-Wang (O-W), Jiang-Sehitoglu (J-S), McDowell and A-V modified hardening rules under various loading conditions (I-K).

The O-W, J-S and McDowell were found to overestimate the ratcheting response of 1045 and $1 \mathrm{Cr} 18 \mathrm{Ni}$ Ti steel samples for various loading paths. Predicted ratcheting curve based on the McDowell model positioned higher than other models in figures 4.7 and 4.8. The predicted ratcheting results based on the J-S model showed closer agreement with experimental data as compared with those of predicted by the $\mathrm{O}-\mathrm{W}$ for axial stress ratios $R>1 / 3$. The $\mathrm{O}-\mathrm{W}$ and consequently $\mathrm{J}-\mathrm{S}$ and McDowell models are significantly influenced by the number of backstress components, $M$, exponent $m_{i}$ and parameters $r_{i}$ and $\gamma_{i}$ defined by segments chosen on the uniaxial stress-strain curve through a tedious and time-consuming trial procedure. 
The J-S, McDowell rules and the proposed model took into account the effect of nonproportionality. The McDowell's modifications on the O-W model is beneficial for cases at which the $\mathrm{O}-\mathrm{W}$ model underestimated the ratcheting strain. The J-S rule on the other hand slightly improved the overestimated ratcheting curves based on the $\mathrm{O}-\mathrm{W}$ model due to terms $d p$ and $m_{i}$ of the dynamic recovery term. The modified model reasonably assessed various multiaxial loading histories through the inclusion of non-proportionality through terms $(2-\bar{n} \cdot \bar{a} /|\bar{a}|)$ and $\langle\bar{n} . \bar{a} /|\bar{a}|\rangle^{1 / 2}$ in the dynamic recovery term of the hardening rule.

The O-W, J-S and McDowell models possessed more complicated structures. The capability of these models were very dependent on the number of backstress components and the magnitude of coefficients. The prediction of ratcheting curves based on the $\mathrm{O}-\mathrm{W}, \mathrm{J}-\mathrm{S}$ and McDowell hardening rules with more number of backstress components, and with larger exponent $m_{i}$ requires longer $\mathrm{CPU}$ time to run the models while time required to predict ratcheting over stress cycles by the modified model is more time efficient mainly due to less complicated hardening rule and holding only three merely material dependent coefficients.

\subsection{Multiaxial ratcheting of SS304 alloys under stress-controlled and combined stress- and strain-controlled histories}

Ratcheting response of SS304 tubular stainless steel samples using nonlinear kinematic hardening rules of Ohno-Wang $(\mathrm{O}-\mathrm{W})$, Chen-Jiao-Kim (C-J-K) and newly modified hardening rule under stress-controlled, and combined stress- and strain-controlled histories, is evaluated. The framework of $\mathrm{O}-\mathrm{W}$ hardening rule was defined on the basis of the critical state of dynamic recovery of backstress. The C-J-K hardening rule further developed the $\mathrm{O}-\mathrm{W}$ rule to include the effect of non-proportionality in ratcheting assessment of materials. The modified hardening rule offered a simple framework including three material dependent coefficients $C, \gamma_{1}$ and $\gamma_{2}$. The modified 
model through the inclusion of $\left\langle d \bar{\varepsilon}_{p} \cdot \bar{a} /|\bar{a}|\right\rangle$ and $(\bar{n} \cdot \bar{a} /|\bar{a}|)$ into its recall term is capable to track different ratcheting directions during multiaxial loading histories.

\subsubsection{SS304 steel samples, testing and multiaxial ratcheting data}

The capability of the O-W, C-J-K and modified A-V hardening rules to assess ratcheting of SS304 steel samples under various non-proportional loading paths was investigated. Multiaxial ratcheting data of three different 304 stainless steel alloys taken from literature [47, 50-51] were conducted under stress-controlled and mixed stress- and strain-controlled conditions. Hassan et al. [47] carried out various multiaxial tests under stress-controlled and mixed stress- and straincontrolled conditions on SS304L tubular specimens of $25 \mathrm{~mm}$ in diameter and thickness of $1.27 \mathrm{~mm}$ using a servohydraulic system. The SS304L samples were annealed in $1050^{\circ} \mathrm{C}$ for an hour and then were quenched slowly in air. The employed loading paths/spectra on steel samples are presented in figures 4.9 and 4.10 (loading paths A1-A3 and B6).

Kang et al. [50] performed ratcheting tests on tubular steel samples of hot-rolled SS304 under different multiaxial stress-controlled paths of A4 and A5 (see figure 4.9). Tubular specimens employed in Kang's experiments possessed inside and outside diameters of $12 \mathrm{~mm}$ and $15 \mathrm{~mm}$, respectively. Tests were conducted using MTS809-25KN machine.

Kim et al. [51] conducted a series of axial-torsional experiments on SS304 tubular samples with inside and outside diameters of $10 \mathrm{~mm}$ and $12.5 \mathrm{~mm}$ under mixed stress- and strain-controlled conditions using an Instron testing machine. The axial-torsional loading histories B1-B5 for Kim's steel samples are presented in figure 4.10. 

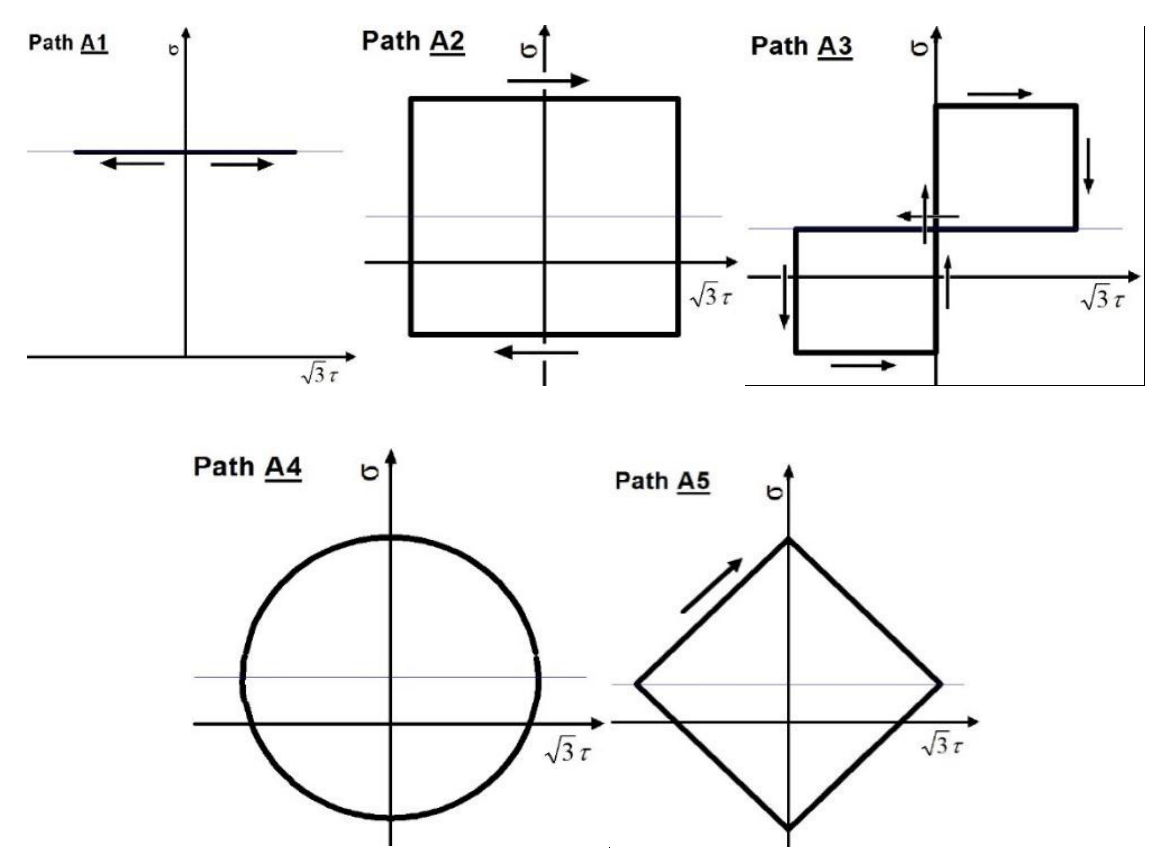

Figure 4.9 Stress-controlled paths.

Under mixed stress- and strain- controlled testing conditions B1-B6, incremental strain and stress respectively were defined through $d \varepsilon_{i j}^{t}$ and $d \sigma_{i j}$. Unknown elements of strain and stress increments $d \sigma_{x y}, d \varepsilon_{x x}^{t}, d \varepsilon_{y y}^{t}$ and $d \varepsilon_{z z}^{t}$ under multiaxial loading conditions were first determined using Symbolic Math Toolbox in MATLAB software. Then the applied cyclic stress and strain values on steel samples were transformed to the stress components in the stress tensor over each increment. The incremental ratcheting strain values were defined using Hooke's law and flow rule through the framework of hardening rules. While, for stress-controlled multiaxial loading histories A1-A5, the components of incremental stress tensor, $d \sigma_{i j}$, are known in each individual increment. 

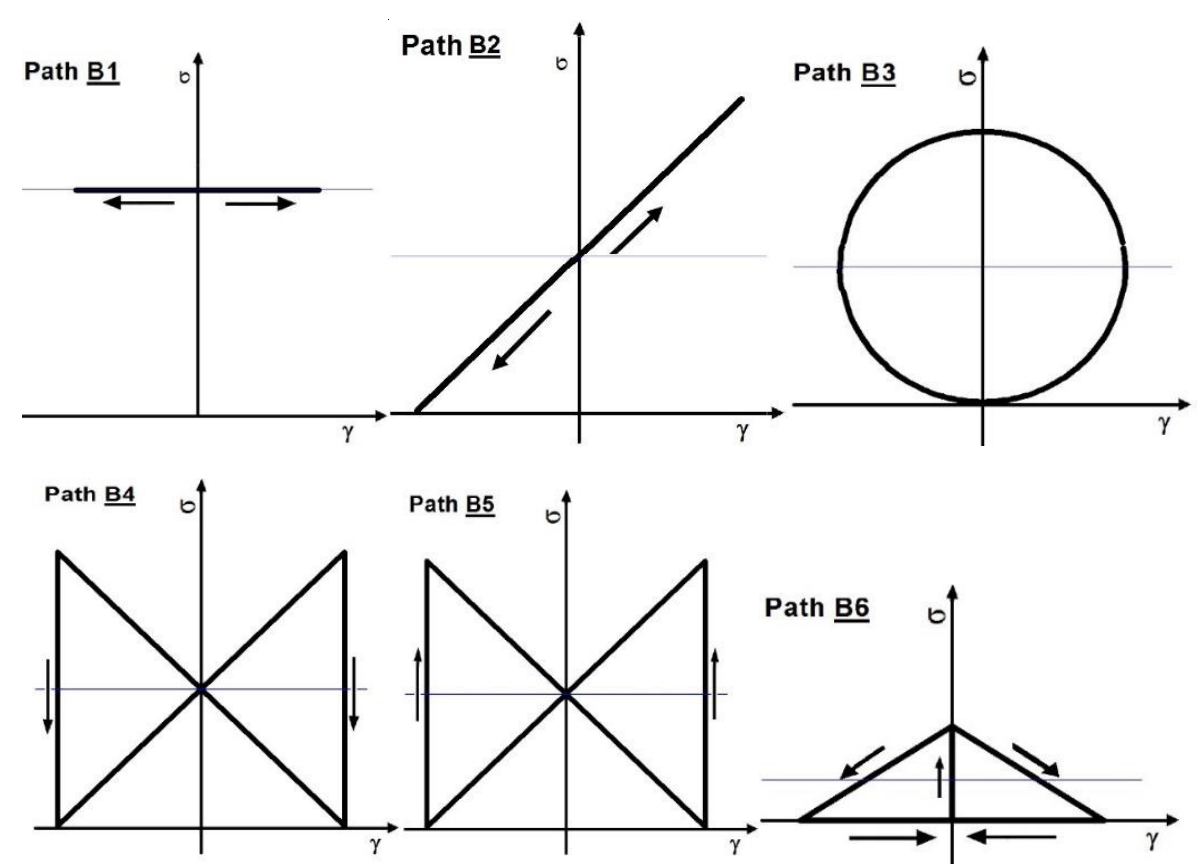

Figure 4.10 Mixed stress- and strain-controlled paths.

Table 4.5 presents ratcheting tests and loading conditions for tubular SS304 samples taken from $[47,50-51]$.

Table 4.5 Multiaxial ratcheting testing for 304 stainless steel samples under various loading paths.

\begin{tabular}{lcccccc}
\hline Materials & Loading Path & $\begin{array}{c}\Delta \boldsymbol{\gamma} / \mathbf{2} \\
(\%)\end{array}$ & $\begin{array}{c}\boldsymbol{\sigma}_{\mathbf{a}} \\
(\mathbf{M P a})\end{array}$ & $\begin{array}{c}\boldsymbol{\sigma}_{\mathbf{m}} \\
(\mathbf{M P a})\end{array}$ & $\begin{array}{c}\boldsymbol{\tau}_{\mathbf{a}} \\
(\mathbf{M P a})\end{array}$ & $\begin{array}{c}\boldsymbol{\tau}_{\mathbf{m}} \\
(\mathbf{M P a})\end{array}$ \\
\hline \multirow{3}{*}{ SS304L [47] } & $\mathbf{A 1}$ & - & 0 & 50 & 115.47 & 0 \\
\cline { 2 - 7 } & $\mathbf{A 2}$ & - & 141.42 & 50 & 81.65 & 0 \\
\hline \multirow{2}{*}{ SS304 [50] } & $\mathbf{A 3}$ & - & 141.42 & 50 & 81.65 & 0 \\
\cline { 2 - 7 } & $\mathbf{A 4}$ & - & 248 & 78 & 143.2 & 0 \\
\hline & $\mathbf{A 5}$ & - & 248 & 78 & 143.2 & 0 \\
\cline { 2 - 7 } & $\mathbf{B 1}$ & 0.866 & 0 & 150 & - & - \\
\cline { 2 - 7 } SS304 [51] & $\mathbf{B 2}$ & 0.866 & 100 & 100 & - & - \\
\cline { 2 - 7 } & $\mathbf{B 3}$ & 0.866 & 100 & 100 & - & - \\
\cline { 2 - 7 } & $\mathbf{B 4}$ & 0.866 & 100 & 100 & - & - \\
\hline SS304L [47] & $\mathbf{B 5}$ & 0.866 & 100 & 100 & - & - \\
\hline
\end{tabular}


In figure 4.9, loading path A1 corresponds to shear stress cycle applied in the presence of a constant axial stress. Path A2 presents a squared-shaped loading condition with fully reversed shear stress cycle and an axial mean stress of $50 \mathrm{MPa}$. Load path A3 is double squared-shaped with a symmetric shear stress cycle in one axis and an axial stress cycle in another loading axis consist of mean value of 50MPa. Loading path A4 represents 90 out-of-phase of sinusoidal symmetric shear stress and asymmetric axial stress spectra. Load path A5 is diamond-shaped with a fully reversed shear stress with a mean axial stress value of 78MPa.

In figure 4.10, loading path B1 corresponds to shear strain cycle applied concurrently with an axial constant stress. Path B2 presents a shear strain cycle and an axial stress with a mean value of $100 \mathrm{MPa}$. Loading path B3 represents 90 degree out-of-phase of sinusoidal shear strain and axial stress spectra. Loading paths B4 and B5 are horizontal butterflies with different directions and with mean axial stresses of 100MPa. Loading path B6 is triangle-shaped with asymmetric axial stress cycle and $0.5 \%$ shear strain amplitude.

Ratcheting strain data of 304 stainless steel alloys of various loading paths were plotted versus multiaxial stress cycles in Fig 4.11. This figure depicts the effect of loading path on the magnitude of ratcheting strain data in SS304 samples achieved at different loading paths, stress amplitudes and mean stresses. 


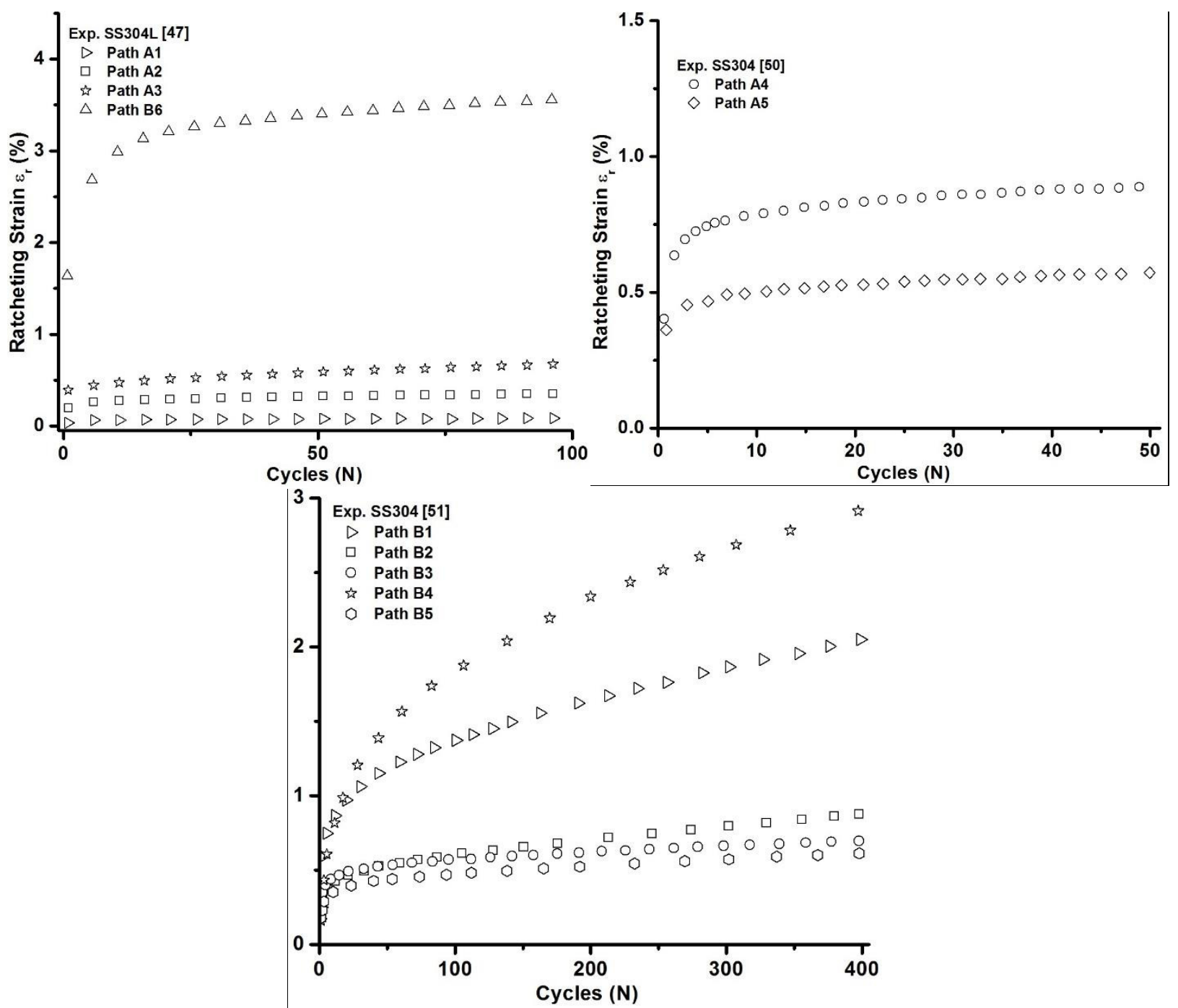

Figure 4.11 Ratcheting strain vs stress cycles for 304 stainless steel alloys under nonproportional stress-controlled conditions (A1-A5) and mixed stress- and strain-controlled conditions (B1-B6).

The calibrating coefficient $\gamma_{2}$ is determined using a uniaxial ratcheting data of 304 stainless steel samples taken from references [47], [50] and [51]. Figure 4.12 shows how $\gamma_{2}$ for three types SS304 is determined using the uniaxial ratcheting data. 

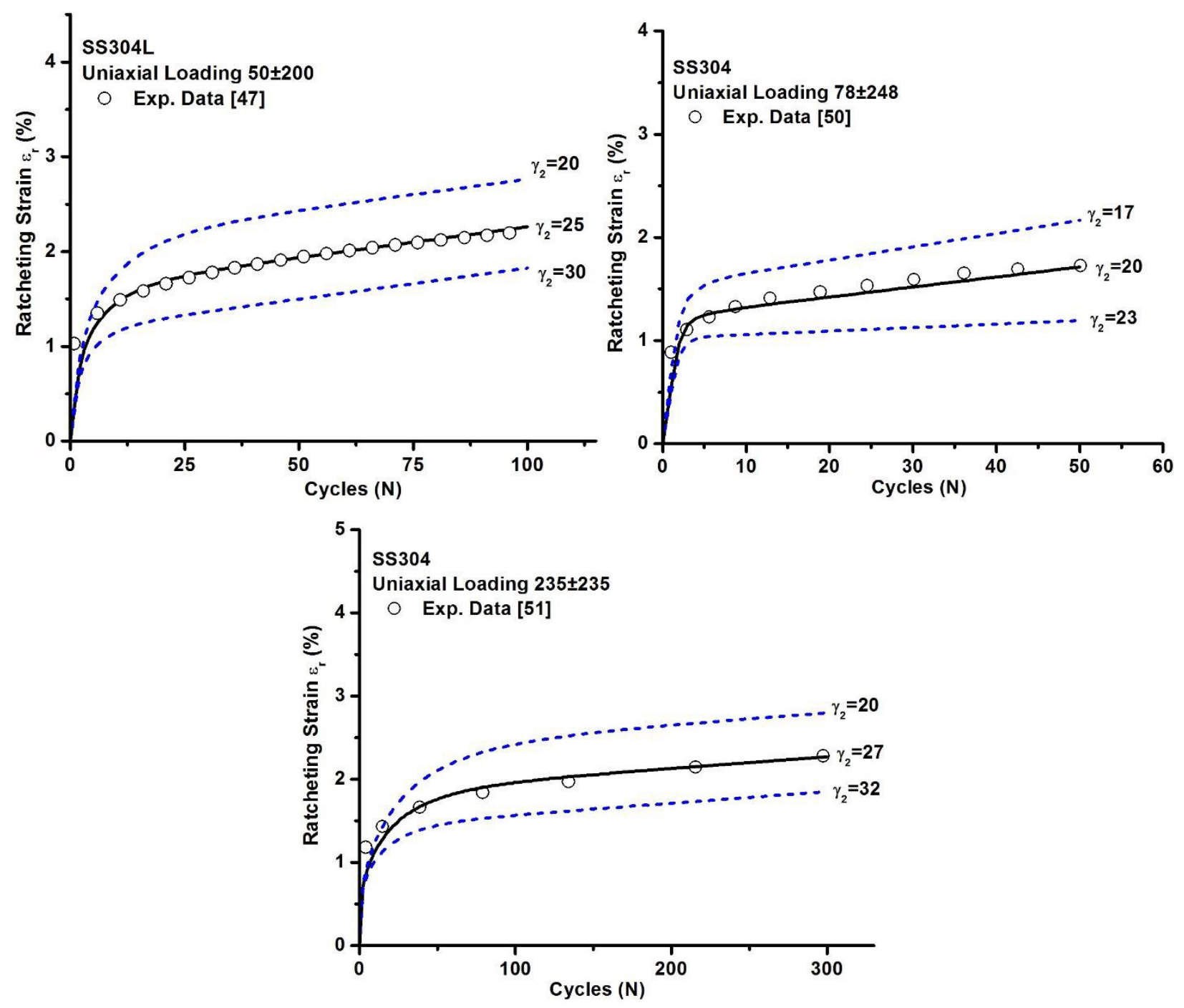

Figure 4.12 Determination of coefficient $\gamma_{2}$ for SS304 samples from uniaxial ratcheting data.

Coefficient $\gamma_{2}$ controls ratcheting rate and regulates the ratcheting response of materials. The coefficients of the O-W, C-J-K and A-V modified hardening rules employed in the current study are listed in Table 4.6. 
Table 4.6 The coefficients of the hardening rules to assess ratcheting response of 304 stainless steel samples.

\begin{tabular}{|c|c|c|}
\hline Materials & Model & Hardening Rule Parameters \\
\hline \multirow{3}{*}{ SS304L [47] } & $\begin{array}{c}\text { Modified } \\
\text { Model }\end{array}$ & $C=55 G P a, \gamma_{1}=190, \gamma_{2}=25$ \\
\hline & $O-W$ Model & $\begin{array}{c}\gamma_{1}=1000, \gamma_{2}=5000, \gamma_{3}=2000, \gamma_{4}=1400, \gamma_{5}=830, \gamma_{6}=340, \gamma_{7}=250, \gamma_{8}=55, \\
r_{1}=18, r_{2}=12, r_{3}=8.5, r_{4}=7.2, r_{5}=5.5, r_{6}=6.6, r_{7}=11, r_{8}=69.5 \\
m_{i}=2\end{array}$ \\
\hline & C-J-K Model & $\begin{array}{c}\gamma_{1}=1000, \gamma_{2}=5000, \gamma_{3}=2000, \gamma_{4}=1400, \gamma_{5}=830, \gamma_{6}=340, \gamma_{7}=250, \gamma_{8}=55 \\
r_{1}=18, r_{2}=12, r_{3}=8.5, r_{4}=7.2, r_{5}=5.5, r_{6}=6.6, r_{7}=11, r_{8}=69.5 \\
m_{i}=2 \quad \chi_{i}=4.8\end{array}$ \\
\hline \multirow{3}{*}{ SS304 [50] } & $\begin{array}{c}\text { Modified } \\
\text { Model }\end{array}$ & $C=80 \mathrm{GPa}, \gamma_{1}=200, \gamma_{2}=20$ \\
\hline & $O-W$ Model & $\begin{array}{c}\gamma_{1}=3448, \gamma_{2}=1527, \gamma_{3}=839.6, \gamma_{4}=210.3, \gamma_{5}=90.87, \gamma_{6}=49.10, \gamma_{7}=31.01, \gamma_{8}=22.65, \\
r_{1}=7.290, r_{2}=66.75, r_{3}=58.91, r_{4}=27.61, r_{5}=15.60, r_{6}=15.90, r_{7}=15.96, r_{8}=117.96 \\
m_{i}=5\end{array}$ \\
\hline & C-J-K Model & $\begin{array}{c}\gamma_{1}=3448, \gamma_{2}=1527, \gamma_{3}=839.6, \gamma_{4}=210.3, \gamma_{5}=90.87, \gamma_{6}=49.10, \gamma_{7}=31.01, \gamma_{8}=22.65, \\
r_{1}=7.290, r_{2}=66.75, r_{3}=58.91, r_{4}=27.61, r_{5}=15.60, r_{6}=15.90, r_{7}=15.96, r_{8}=117.96 \\
m_{i}=5 \quad \chi_{i}=1.3\end{array}$ \\
\hline \multirow{3}{*}{ SS304 [51] } & $\begin{array}{c}\text { Modified } \\
\text { Model }\end{array}$ & $C=75 \mathrm{GPa}, \gamma_{1}=190, \gamma_{2}=21$ \\
\hline & O-W Model & $\begin{array}{c}\gamma_{1}=2000, \gamma_{2}=1000, \gamma_{3}=500, \gamma_{4}=250, \gamma_{5}=167, \gamma_{6}=100, \gamma_{7}=62.5, \gamma_{8}=31.25, \\
r_{1}=60, r_{2}=60, r_{3}=40, r_{4}=50, r_{5}=27, r_{6}=24, r_{7}=17, r_{8}=52 \\
m_{i}=6\end{array}$ \\
\hline & C-J-K Model & $\begin{array}{c}\gamma_{1}=2000, \gamma_{2}=1000, \gamma_{3}=500, \gamma_{4}=250, \gamma_{5}=167, \gamma_{6}=100, \gamma_{7}=62.5, \gamma_{8}=31.25 \\
r_{1}=60, r_{2}=60, r_{3}=40, r_{4}=50, r_{5}=27, r_{6}=24, r_{7}=17, r_{8}=52 \\
m_{i}=6 \quad \chi_{i}=2.9\end{array}$ \\
\hline
\end{tabular}

\subsubsection{Predicted ratcheting of SS304 steel by O-W, C-J-K and A-V modified hardening rules under stress-controlled and combined stress- and strain-controlled histories}

The multiaxial ratcheting response of 304 stainless steel alloys under stress-controlled, and mixed stress- and strain-controlled conditions were predicted by means of the O-W, C-J-K and A$\mathrm{V}$ modified kinematic hardening rules. The experimental and the predicted ratcheting strain values based on the modified model (Eq. (3.8)), and the hardening rules of O-W (Eq. (2.8)) and C-J-K (Eq. (2.13)) for 304 stainless steel alloys tested under various stress-controlled and combined stress- and strain-controlled paths are presented in figures 4.13 and 4.14, respectively. Ratcheting 
strains progressed in the direction of the axial mean stress with a decay in the accumulated ratcheting rate over multiaxial stress cycles for both loading conditions. The capability of the OW, C-J-K and modified hardening rules in ratcheting assessment of 304 stainless steel samples under five different stress-controlled loading paths A1-A5 was assessed as the predicted ratcheting curves were compared with those of experimentally reported values in Fig. 4.13. Experimental ratcheting data in this figure shows consistently good agreements with those of predicted curves based on the modified hardening rule for multiaxial loading paths A2, A3, A4, and A5 beyond first few cycles of 10-20. The predicted ratcheting curve for loading path A1 however overestimated the ratcheting data in this figure. A larger overestimation has been reported as ratcheting of loading path A1 was predicted by the first and second O-W, Abdel Karim -Ohno, and Kang models in ref. [72].

The predicted ratcheting curves based on modified model were found in the closer agreement with those of experimentally reported which is attributed to inclusion of terms $\left\langle d \bar{\varepsilon}_{p} \cdot \bar{a} /|\bar{a}|\right\rangle$ and $(\bar{n} \cdot \bar{a} /|\bar{a}|)$ in the recall term in the framework of proposed rule. The replacement of accumulated plastic strain increment $d p$ with $\left\langle d \bar{\varepsilon}_{p} \cdot \bar{a} /|\bar{a}|\right\rangle$ in the dynamic recovery term shifts down the overall magnitude of predicted ratcheting curve under multiaxial loading, since components $d \bar{\varepsilon}_{p}$ and $\bar{a} /|\bar{a}|$ hold different direction and consequently their projection presented in the MaCaulay brackets induces a slower rate of ratcheting strain over stress cycles. In the meantime, material dependent coefficient $\gamma_{2}$ first determined using uniaxial ratcheting response, is incrementally calibrated by $(2-\bar{n} \cdot \bar{a} /|\bar{a}|)$ consistent with applied multiaxial loading. This term, taking values between 1 and 3 under multiaxial loading, results in lower ratcheting rate.

The non-proportionality term $(\bar{n} \cdot \bar{a} /|\bar{a}|)$ once again was employed in the form of $\langle\bar{n} \cdot \bar{a} /|\bar{a}|\rangle^{1 / 2}$ multiplying vector $\bar{b}$ to prevent the modified model experiencing plastic shakedown. The non-proportionality term $(\bar{n} \cdot \bar{a} /|\bar{a}|)$ takes values between -1 and 1 under multiaxial loading 
histories. Term $\langle\bar{n} \cdot \bar{a} /|\bar{a}|\rangle^{1 / 2}$ produces values between 0 and 1 under multiaxial loading reduces to 1 for uniaxial loading case.

For loading paths A1-A5, the O-W model overestimated ratcheting response of 304 stainless steel samples in figure 4.13. Chen and Jiao [29] have further concluded that the O-W model overestimated the ratcheting response of cyclically hardened materials under multiaxial loading conditions. This overestimation was earlier attributed to the absence non-proportionality factor to control the evolution of ratcheting over complex stress cycles [36]. Chen et al. [30] further modified the dynamic recovery term through $\left.\left\langle\bar{n} .\left(\bar{a}_{i} /\left|\bar{a}_{i}\right|\right)\right\rangle\right\rangle^{x_{i}}$ to incorporate the loading nonproportionality into the hardening rule. Term $\left\langle\bar{n} . \bar{a}_{i} /\left|\bar{a}_{i}\right|\right\rangle$ takes different values of either zero or a value less than 1 under non-proportional loading and the terms in the MaCaulay brackets $\left.\left\langle\bar{n} .\left(\bar{a}_{i} /\left|\bar{a}_{i}\right|\right)\right\rangle\right\rangle^{\chi_{i}}$ encounter the loading non-proportionality in ratcheting assessment of materials for exponent $\chi_{i}>0$.

Based on the C-J-K model, exponent $\chi_{i}$ was first calibrated based on a set of multiaxial ratcheting data and then was employed for various loading paths. For 304L stainless steel samples [47] exponent $\chi_{i}=4.8$ was initially calibrated to coincide the ratcheting curve predicted by the CJ-K model with those of experimentally obtained under loading Path A1. This value was then employed to predict the ratcheting under stress-controlled paths A2 and A3 as well as combined stress- and strain-controlled path B6. The predicted ratcheting curves for loading paths A1-A3 and B6 have positioned closer to experimental data as compared with predicted curves by the O-W mainly due to implementing term $\left\langle\bar{n} .\left(\bar{a}_{i} /\left|\bar{a}_{i}\right|\right)\right\rangle^{x_{i}}$. The C-J-K model is yet to closely follow the trend of ratcheting data of 304L in loading paths A1-A3 and B6. To predict ratcheting of 304 stainless steel under loading paths A4 and A5 [50] exponent $\chi_{i}=1.3$ was first defined to predict ratcheting of SS304 in agreement with experiment data of path A4. The C-J-K hardening rule with 
the same value of $\chi_{i}$ failed to well predict ratcheting of loading path A5 as close as the model predicted ratcheting under loading path A4.
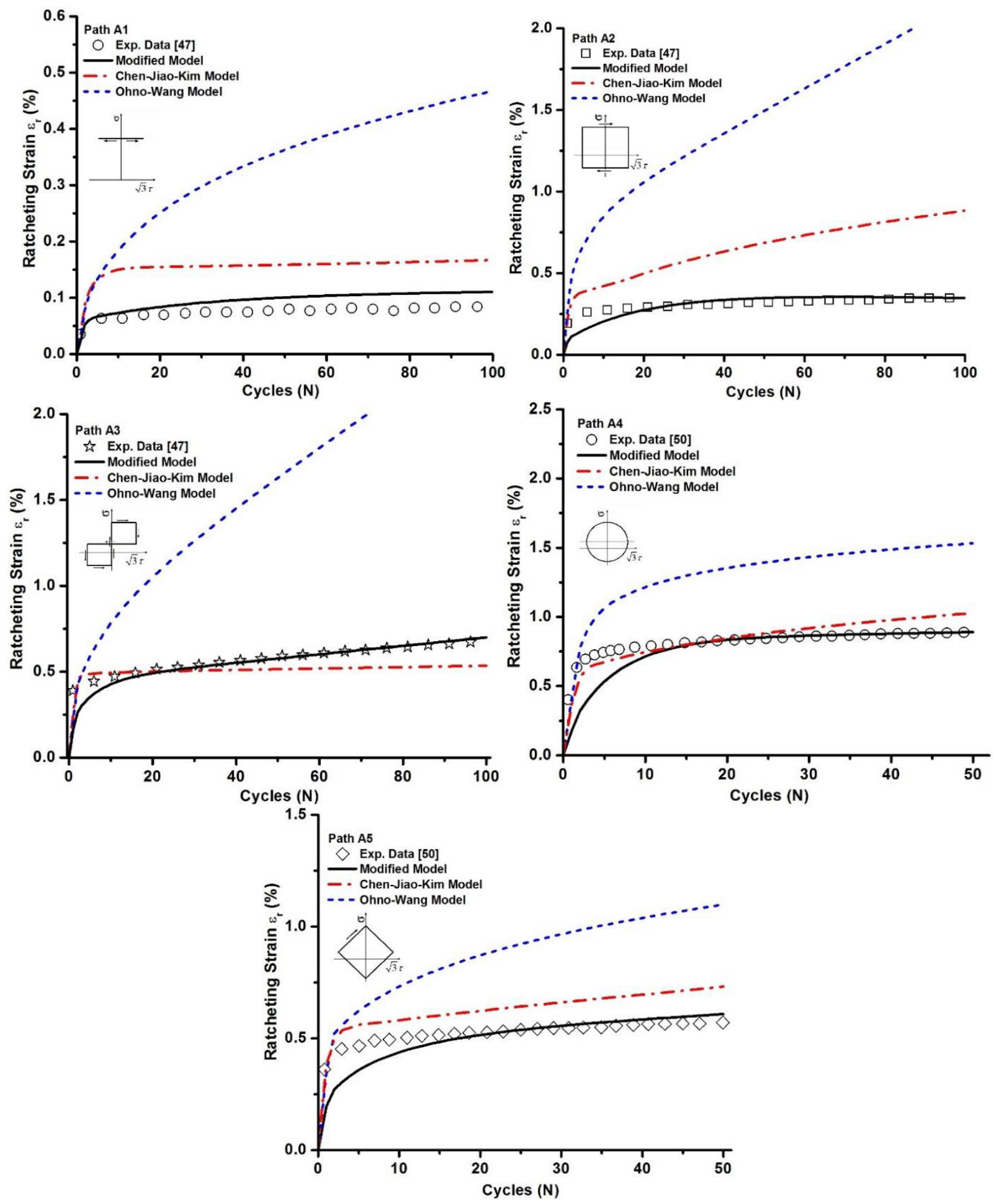

Figure 4.13 Experimental and predicted ratcheting strain values of 304 stainless steel samples based on the $\mathrm{O}-\mathrm{W}, \mathrm{C}-\mathrm{J}-\mathrm{K}$ and $\mathrm{A}-\mathrm{V}$ modified hardening rules under various stress-controlled paths A1-A5. 
Figure 4.14 presents the predicted ratcheting curves of 304 stainless steel samples tested under combined stress- and strain-controlled histories B1-B6. The ratcheting curves were predicted based on the $\mathrm{O}-\mathrm{W}$ and $\mathrm{C}-\mathrm{J}-\mathrm{K}$ hardening rules considering eight backstress components $M=8$.

The predicted ratcheting curves based on the $\mathrm{O}-\mathrm{W}$ model in this figure largely overestimated the experimental ratcheting data of SS304 samples [51] under B1-B5 loading histories. The $\mathrm{O}-\mathrm{W}$ hardening rule underestimated the ratcheting of 304L sample tested under triangular loading path B6 [47] for initial 12-15 cycles and then largely overestimated the ratcheting trend as the number of cycle progressed. To predict the ratcheting curves of SS304 [51] under various paths, $\chi_{i}=2.9$ was calibrated based on ratcheting data of B1. The C-J-K model, employing this value for other loading paths B2-B5, improved the overestimation predicted earlier by the $\mathrm{O}-\mathrm{W}$ model. The predicted ratcheting curves for loading paths B1 and B4 were found in good agreements with experimental data reported by Kim et al. [51]. The C-J-K model with the same multiaxial factor $\chi_{i}$ showed a noticeable deviation of predicted ratcheting curves for paths $\mathrm{B} 2, \mathrm{~B} 3$, and B5. The ratcheting of B6 sample predicted by the C-J-K showed some underestimation in consistent with what reported by Abdel-Karim [72].

Figure 4.14 also shows how closely the ratcheting curves predicted based on the modified model are positioned next to the experimental values for loading paths B1-B5. The modified model underestimated the ratcheting strain of 304L stainless steel samples under loading path B6. A larger underestimation in ratcheting was also achieved for B6 loading path when predicted based on the Abdel-Karim and Chen-Jiao models [72]. 

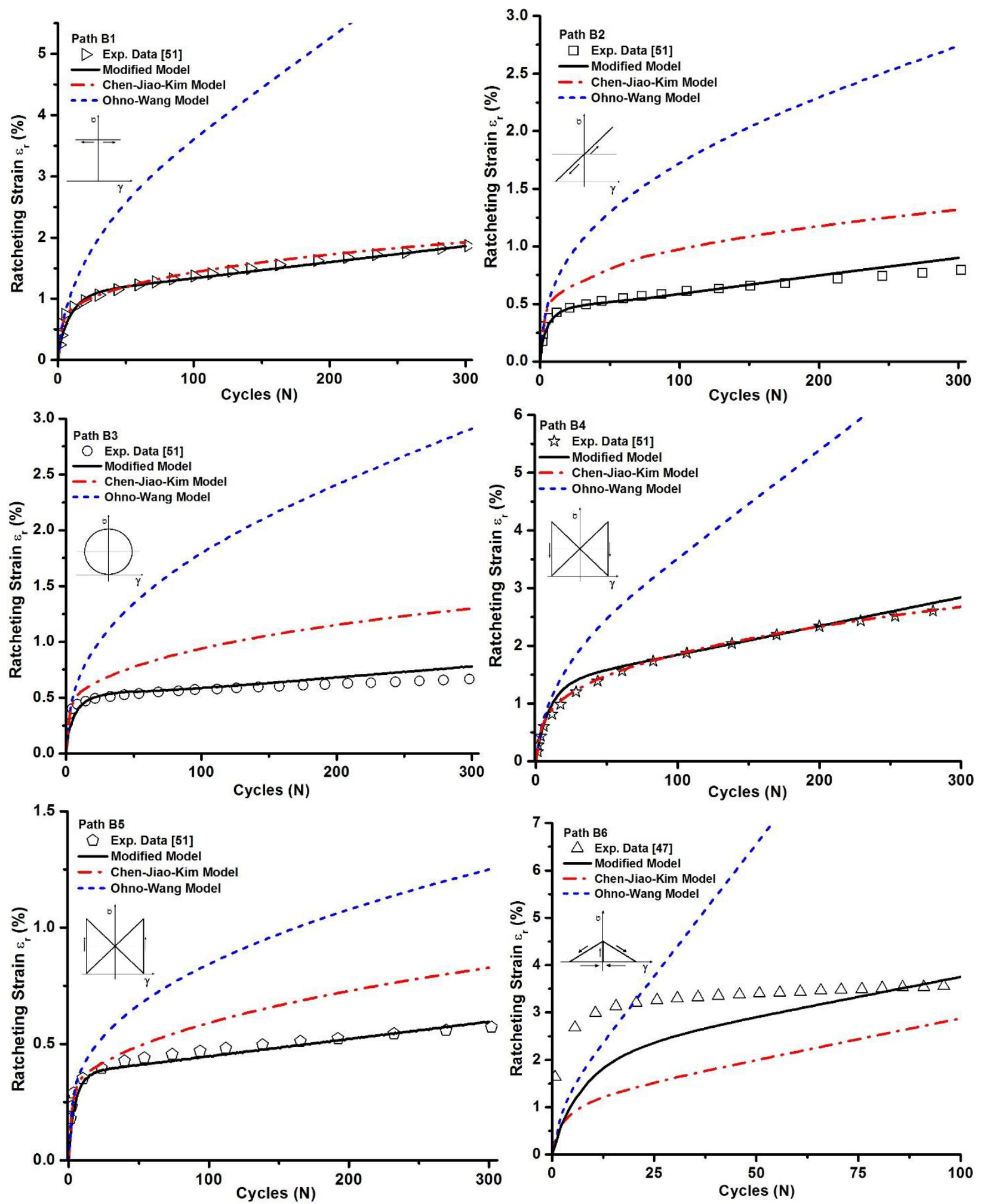

Figure 4.14 Experimental and predicted ratcheting strain values of 304 stainless steel samples based on the O-W, C-J-K and A-V modified hardening rules under combined stress- and straincontrolled tests B1-B6. 
The O-W model overestimated the ratcheting response of 304 stainless steel samples under both stress-controlled and combined stress- and strain-controlled paths. Larger values of $m_{i}$ in equation (2.8) suppressed the rate of accumulated ratcheting over multiaxial stress cycles. Constants $m_{i}, r_{i}$ and $\gamma_{i}$ in the kinematic hardening rules of O-W [19, 20] and C-J-K [30] are defined by segments chosen on the uniaxial stress-strain and uniaxial ratcheting curves through a tedious and time-consuming trial procedure. The persistent overestimation of ratcheting curves predicted based on the $\mathrm{O}-\mathrm{W}$ model in figures 4.13 and 4.14 was attributed to the absence of the nonproportionality factor in the framework of the O-W. The C-J-K model addressed this shortcoming of the O-W model by incorporating term $\left\langle\bar{n} \cdot\left(\bar{a}_{i} /\left|\bar{a}_{i}\right|\right)\right\rangle{ }^{\chi_{i}}$ into the dynamic recovery term to include the effect of loading non-proportionality. The C-J-K model showed a closer agreement of the predicted ratcheting curves of SS304 steel samples to experimental data as compared to those curves predicted by the $\mathrm{O}-\mathrm{W}$ model. Exponent $\chi_{i}$ in the C-J-K model once calibrated based on a multiaxial ratcheting data set, hardly became applicable for other multiaxial loading histories. For instance, the calibrated $\chi_{i}$ for SS304 under loading path A4 failed to result in good prediction for loading path A5. Similarly, the $\chi_{i}$ defined for loading path B1 is yet to well predict ratcheting for tests with loading paths B2, B3 and B5 of the same steel alloy.

The modified hardening rule was found consistently capable to predict ratcheting of 304 steel alloys under various stress-controlled and combined stress- and strain-controlled tests. The overestimation in predicting the ratcheting of steel alloys for loading paths A1 and B6 as compared with experimental data reported by researchers in reference [49] was attributed to the distortion of the yield surface affecting the ratcheting response in 304 samples.

The O-W and C-J-K models were significantly influenced by the number of backstress components, $M$, exponent $m_{i}$ and parameters $r_{i}$ and $\gamma_{i}$ defined by segments chosen on the uniaxial stress-strain curve through a tedious and time-consuming trial procedure. Both the C-J-K and modified models took into account the effect of non-proportionality. The C-J-K rule improved the 
overestimated ratcheting curves predicted by the $\mathrm{O}-\mathrm{W}$ model as a non-proportionality factor was introduced into the dynamic recovery term.

The modified model reasonably assessed various loading histories through the inclusion of non-proportionality term $(\bar{n} \cdot \bar{a} /|\bar{a}|)$ in the dynamic recovery term of the hardening rule. The capability of the O-W and C-J-K models was very much dependent on the number of backstress components and how the coefficients are chosen/determined. The prediction of ratcheting curves based on the O-W and C-J-K hardening rules with more number of backstress components, and with larger exponent $m_{i}$ required longer CPU time to run the models while time required to predict ratcheting over stress cycles by the modified model was insignificant mainly due to less complicated hardening rule and its limited number of coefficients.

\subsection{Ratcheting assessment of SS304 steel under multiaxial step-loading conditions}

The present study predicts ratcheting response of 304 tubular stainless steel samples undergoing multiaxial step-loading histories by means of nonlinear kinematic hardening rules of Ohno-Wang (O-W), Chen-Jiao-Kim (C-J-K) and the modified Ahmadzadeh-Varvani (A-V). The influence of load steps on ratcheting progress over multiaxial load cycles in 304 steel samples was evidential for five different loading paths discussed in this section. The prior load step in these paths greatly affected the ratcheting buildup in subsequent steps. The predicted ratcheting curves and generated axial-torsional hysteresis loops based on the modified are also compared with experimentally obtained hysteresis loops of SS304 steel samples over loading steps.

\subsubsection{SS304 sample, testing and multiaxial ratcheting data}

Ratcheting data of 304 stainless steel samples tested under various multiaxial step-loading spectra were taken from literature $[43,50]$ to assess the capability of the O-W, C-J-K and modified A-V hardening rules in predicting ratcheting curves. 
Ratcheting tests on tubular steel samples of hot-rolled SS304 were performed under different multiaxial stress-controlled paths of A-E as presented in figure 4.15.
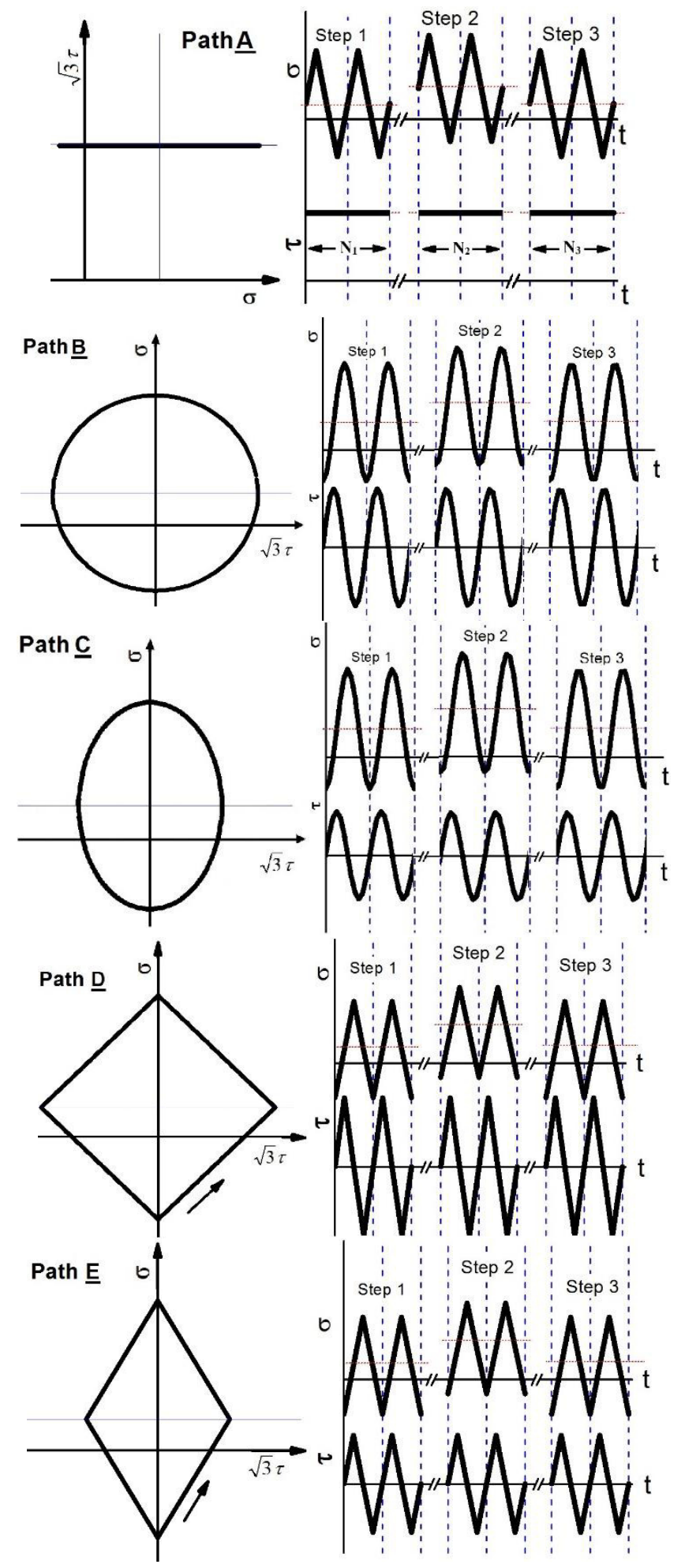

Figure 4.15 Step-loading paths and multiaxial testing histories. 
Tubular specimens in these experiments possessed inside and outside diameters of $12 \mathrm{~mm}$ and $15 \mathrm{~mm}$, respectively and ratcheting tests were conducted using MTS809-25KN testing machine. Table 4.7 presents ratcheting tests and loading conditions for tubular SS304 samples taken from [43].

Table 4.7 Step-loading multiaxial ratcheting testing of SS304 samples under various loading paths [50].

\begin{tabular}{|c|c|c|c|c|c|c|c|c|c|}
\hline \multirow{2}{*}{ Path } & \multicolumn{3}{|c|}{ Step 1} & \multicolumn{3}{|c|}{ Step 2} & \multicolumn{3}{|c|}{ Step 3} \\
\hline & $\begin{array}{c}\sigma_{\mathrm{m} 1} \pm \sigma_{\mathrm{a} 1} \\
(\mathrm{MPa})\end{array}$ & $\begin{array}{c}\tau_{\mathrm{m} 1} \pm \tau_{\mathrm{a} 1} \\
(\mathrm{MPa})\end{array}$ & $\mathrm{N}_{1}$ (Cycle) & $\begin{array}{c}\sigma_{\mathrm{m} 2} \pm \sigma_{\mathrm{a} 2} \\
(\mathrm{MPa})\end{array}$ & $\begin{array}{c}\tau_{\mathrm{m} 2} \pm \tau_{\mathrm{a} 2} \\
(\mathrm{MPa})\end{array}$ & $\mathrm{N}_{2}$ (Cycle) & $\begin{array}{c}\sigma_{\mathrm{m} 3} \pm \sigma_{\mathrm{a} 3} \\
(\mathrm{MPa})\end{array}$ & $\begin{array}{c}\tau_{\mathrm{m} 3} \pm \tau_{\mathrm{a} 3} \\
(\mathrm{MPa})\end{array}$ & $\mathrm{N}_{3}$ (Cycle) \\
\hline $\mathbf{A}$ & $78 \pm 248$ & $67.55 \pm 0$ & 50 & $117 \pm 248$ & $67.55 \pm 0$ & 50 & $78 \pm 248$ & $67.55 \pm 0$ & 20 \\
\hline B & $78 \pm 248$ & $0 \pm 143.2$ & 50 & $117 \pm 248$ & $0 \pm 143.2$ & 50 & $78 \pm 248$ & $0 \pm 143.2$ & 20 \\
\hline C & $78 \pm 248$ & $0 \pm 71.6$ & 50 & $117 \pm 248$ & $0 \pm 71.6$ & 50 & $78 \pm 248$ & $0 \pm 71.6$ & 20 \\
\hline D & $78 \pm 248$ & $0 \pm 143.2$ & 50 & $117 \pm 248$ & $0 \pm 143.2$ & 50 & $78 \pm 248$ & $0 \pm 143.2$ & 20 \\
\hline $\mathbf{E}$ & $78 \pm 248$ & $0 \pm 71.6$ & 50 & $117 \pm 248$ & $0 \pm 71.6$ & 50 & $78 \pm 248$ & $0 \pm 71.6$ & 20 \\
\hline
\end{tabular}

In figure 4.15, loading path A corresponds to axial stress cycle applied concurrently with a constant shear stress. Loading paths B and C represent 90 out-of-phase of sinusoidal shear stress and axial stress axes which result in circular and elliptical paths respectively possessing 143.2MPa and 71.6MPa as shear stress amplitudes. Loading paths D and E are diamond-shaped with a fully reversed shear stress with stress amplitudes of respectively $143.2 \mathrm{MPa}$ and $71.6 \mathrm{MPa}$, and asymmetric axial stress cycles. Paths A-E possess histories consist of three load steps with lowhigh-low sequences at which torsional loading was kept unchanged over three loading steps while the asymmetric axial axes in these histories included mean stresses of $78 \mathrm{MPa}, 117 \mathrm{MPa}$, and $78 \mathrm{MPa}$ over the first, the second, and the third steps of loading spectra, respectively. The axial and torsional stress amplitudes stayed unchanged at 248MPa over loading steps.

Ratcheting strain data of 304 stainless steel samples of various loading paths were plotted versus multiaxial loading steps in Fig 4.16. This figure shows how influential the effect of loading 
path, loading steps and its sequence on the magnitude of ratcheting strain data in SS304 samples is.

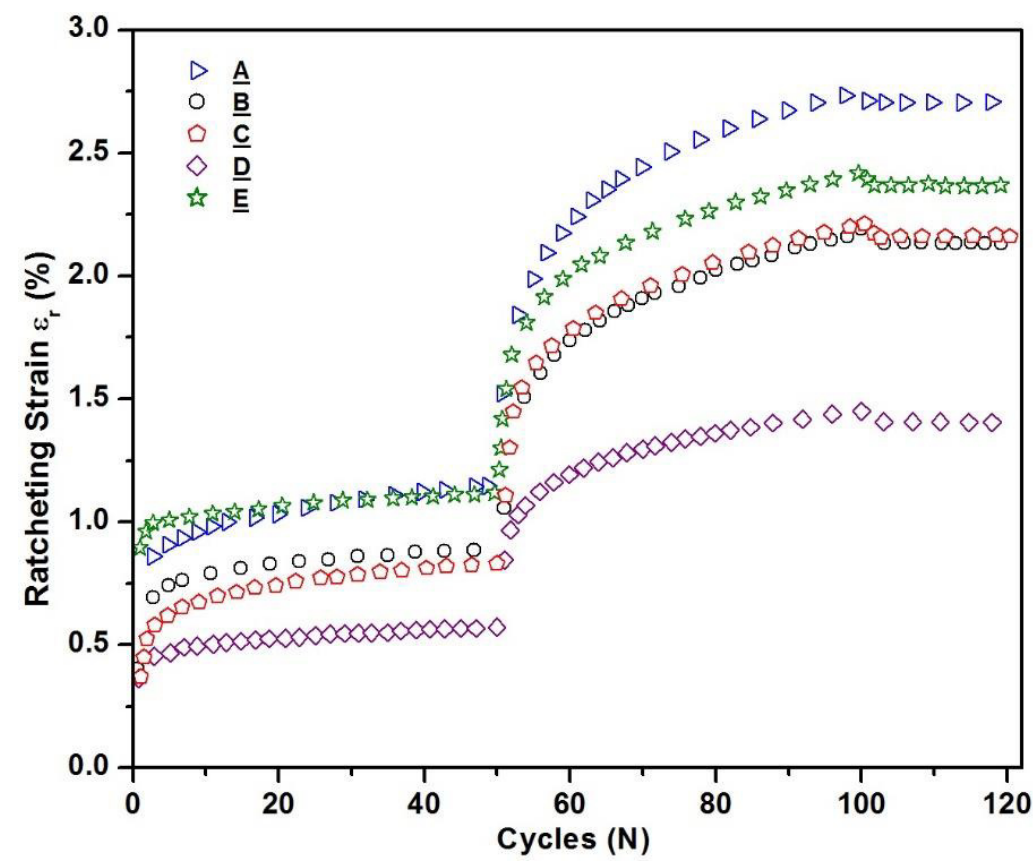

Figure 4.16 Ratcheting strain vs stress cycles for 304 stainless steel samples tested under multiaxial step-loading histories A-E [50].

Coefficients of the O-W, C-J-K and modified A-V hardening rules employed in the current study are listed in Table 4.8 .

Table 4.8 Coefficients of the hardening rules to assess ratcheting response of 304 stainless steel samples.

Model Hardening Rule Parameters

Modified Model

$C=80 \mathrm{GPa}, \gamma_{1}=200, \gamma_{2}=20$

O-W Model

$\gamma_{1}=3448, \gamma_{2}=1527, \gamma_{3}=839.6, \gamma_{4}=210.3, \gamma_{5}=90.87, \gamma_{6}=49.10, \gamma_{7}=31.01, \gamma_{8}=22.65$,

$r_{1}=7.290, r_{2}=66.75, r_{3}=58.91, r_{4}=27.61, r_{5}=15.60, r_{6}=15.90, r_{7}=15.96, r_{8}=117.96$

$m_{i}=5$

C-J-K Model

$\gamma_{1}=3448, \gamma_{2}=1527, \gamma_{3}=839.6, \gamma_{4}=210.3, \gamma_{5}=90.87, \gamma_{6}=49.10, \gamma_{7}=31.01, \gamma_{8}=22.65$,

$r_{1}=7.290, r_{2}=66.75, r_{3}=58.91, r_{4}=27.61, r_{5}=15.60, r_{6}=15.90, r_{7}=15.96, r_{8}=117.96$

$m_{i}=5 \quad \chi_{i}=1.3$ 


\subsubsection{Multiaxial ratcheting of SS304 over loading steps by the modified model}

Ratcheting response of 304 stainless steel samples undergoing multiaxial step-loading histories A-E was predicted by means of the modified hardening rule and was compared with experimental ratcheting data over loading steps in figures $4.17 \mathrm{a}-4.21$. The stress amplitude over step-loading histories remained constant while mean stress varied with low-high-low sequences. The experimentally obtained tensile strain-shear strain $(\varepsilon-\gamma)$ hysteresis loops and those predicted by the modified model over three low-high-low loading steps are presented in parts (b) and (c) of figures 4.17-4.21.

In figure 4.17, loading path A with a constant stress amplitude of 248MPa and low-highlow mean stress sequence of $78 \mathrm{MPa}, 117 \mathrm{MPa}, 78 \mathrm{MPa}$ resulted in ratcheting strain to progress in the direction of axial mean stress over the first and the second steps while it showed a change in direction of ratcheting accumulation over the third step. The predicted ratcheting curve closely agreed with experimental data over the first and third steps in figure 4.17a however a deviation of up to $7 \%$ between the predicted and experimental ratcheting values is evidenced within the second step. In the third step the modified model enabled the ratcheting strain values to lower agreeable with the experimental data as a result of a drop in mean stress level. The change in direction of predicted ratcheting curve over the third step of loading path A verifies the capability of the model. The experimental hysteresis loops $(\varepsilon-\gamma)$ in figure $4.17 \mathrm{~b}$ and those of predicted in figure $4.17 \mathrm{c}$ closely agreed over low-high-low loading steps. These figures verify the presence of the shear ratcheting strain for non-zero shear mean stress of $\tau_{\mathrm{m}}=67.55 \mathrm{MPa}$. 

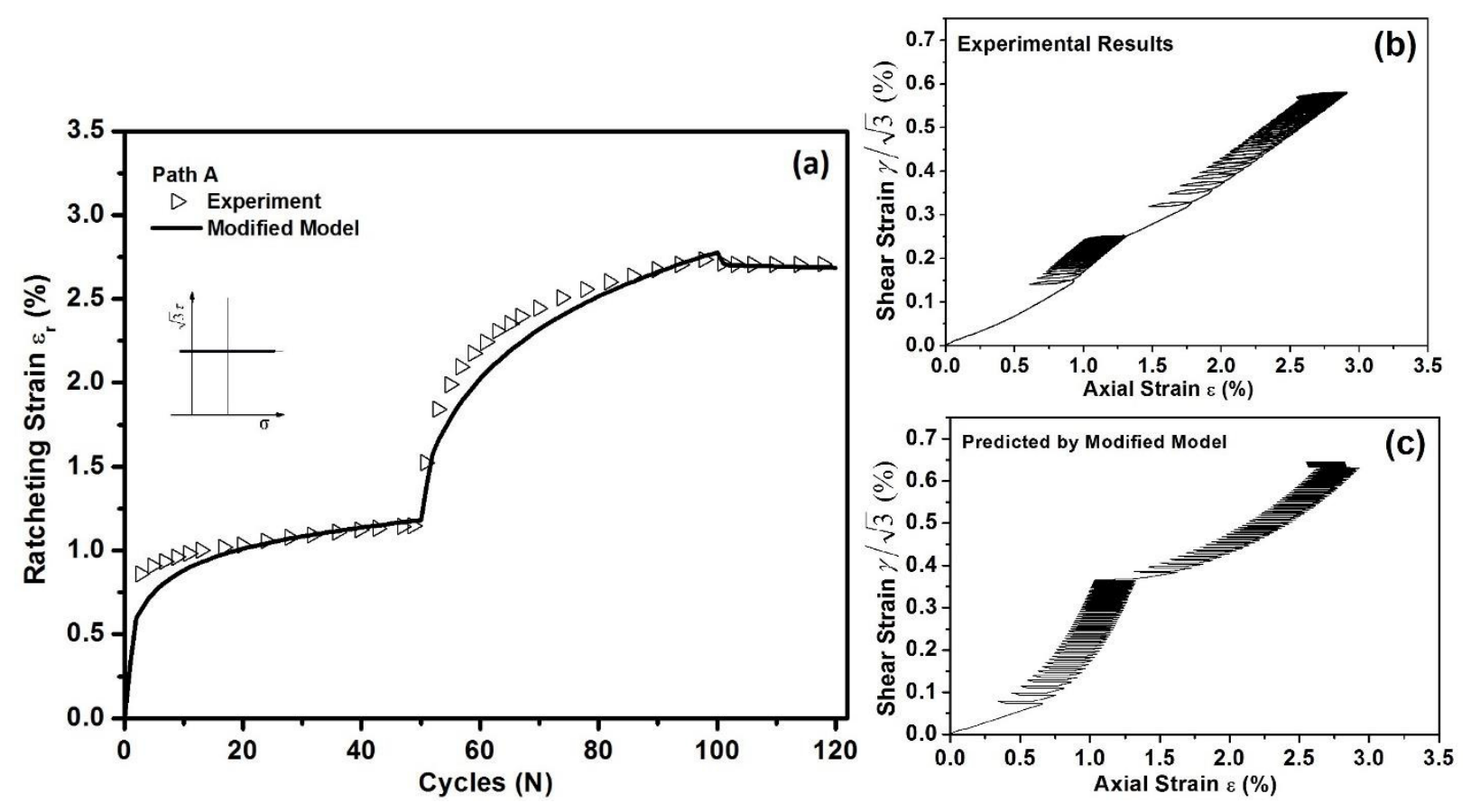

Figure 4.17 (a) Experimental ratcheting data [50] vs predicted ratcheting curves based on the modified model, (b) experimental hysteresis loops [50] ( $\varepsilon-\gamma)$, and (c) predicted hysteresis loops $(\varepsilon-\gamma)$ over load steps for history A.

The predicted ratcheting curves of SS304 steel sample undergoing circular loading path B with a constant stress amplitude of $248 \mathrm{MPa}$ and mean stresses $78 \mathrm{MPa}, 117 \mathrm{MPa}$, and $78 \mathrm{MPa}$ over three load steps was compared with those of experimentally reported in Fig. 4.18a. The predicted ratcheting curves agreed with experimental data over the first step and third step and they showed an underestimation as high as $5 \%$ as compared with experimental data within the second step. The experimental axial-shear ratcheting hysteresis loops in figure $4.18 \mathrm{~b}$ agreed with those of predicted by the modified model in figure $4.18 \mathrm{c}$. These figures show the dominancy of ratcheting along axial direction due to the applied axial mean stresses and a minor shear ratcheting progress as shear mean stresses were absent over load steps. The evidence of minor shear ratcheting progress in the absence of applied shear mean stresses have been also reported by Kang et al. [50, 73]. 

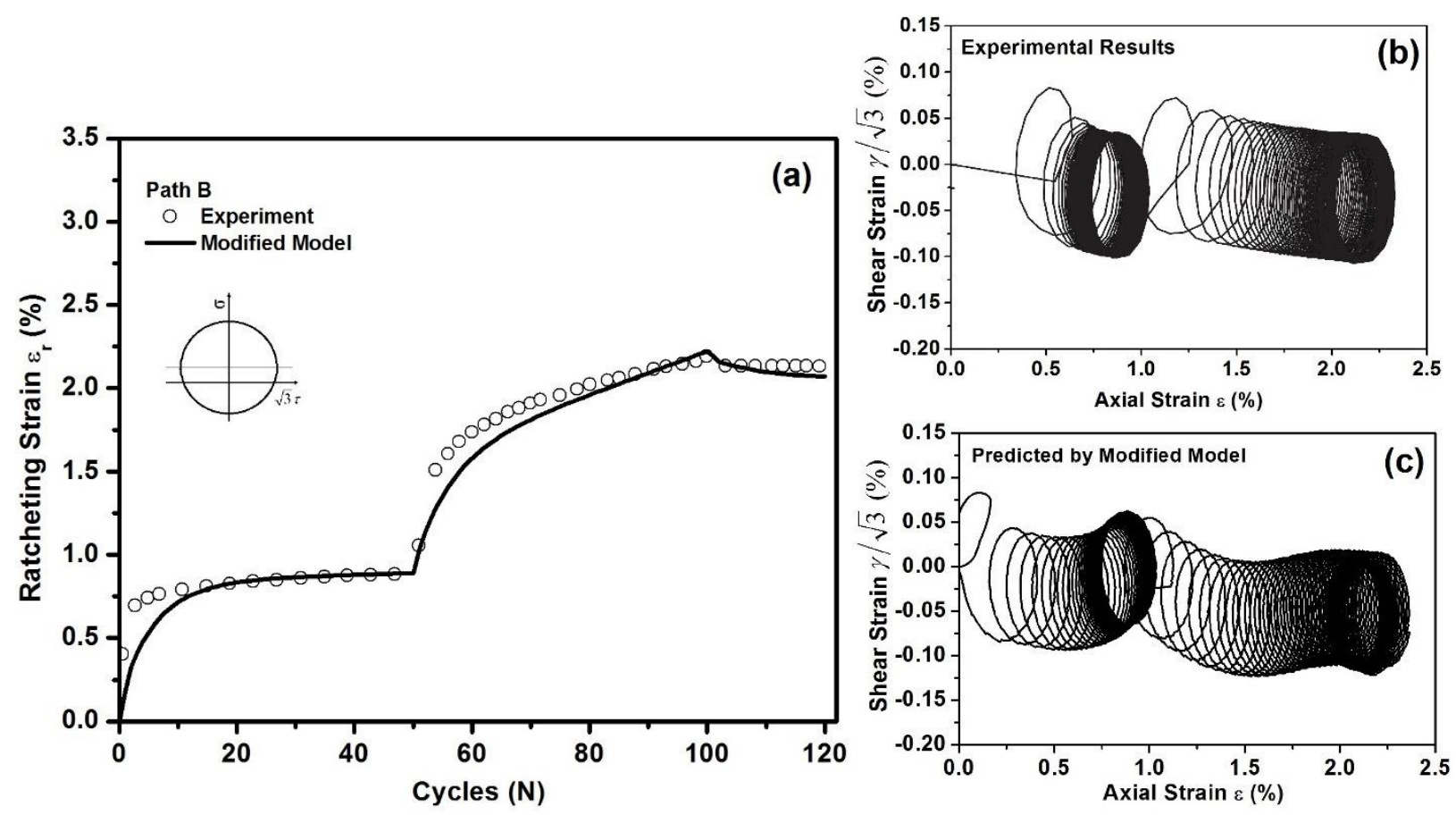

Figure 4.18 (a) Experimental ratcheting data [50] vs predicted ratcheting curves based on the modified model, (b) experimental hysteresis loops [50] ( $\varepsilon-\gamma)$, and (c) predicted hysteresis loops $(\varepsilon-\gamma)$ over load steps for history B.

Figure 4.19a plots both experimentally obtained ratcheting strain data of 304 stainless steel and those of predicted curves over three load steps of history $\mathrm{C}$ consists of the constant stress amplitude of $248 \mathrm{MPa}$ and mean stresses of $78 \mathrm{MPa}, 117 \mathrm{MPa}, 78 \mathrm{MPa}$. The predicted ratcheting curves in this figure underestimated the ratcheting for the first 10-15 cycles and closely agreed with experimental data as the number of cycle advanced in step 1 . The predicted ratcheting curve in the middle step fell below the experimental data with a magnitude of $7 \%$. At the third step both predicted and experimental ratcheting data collapsed next to each other. The experimental and predicted hysteresis loops $(\varepsilon-\gamma)$ in figures $4.19 \mathrm{~b}$ and $4.19 \mathrm{c}$ showed close agreements. 

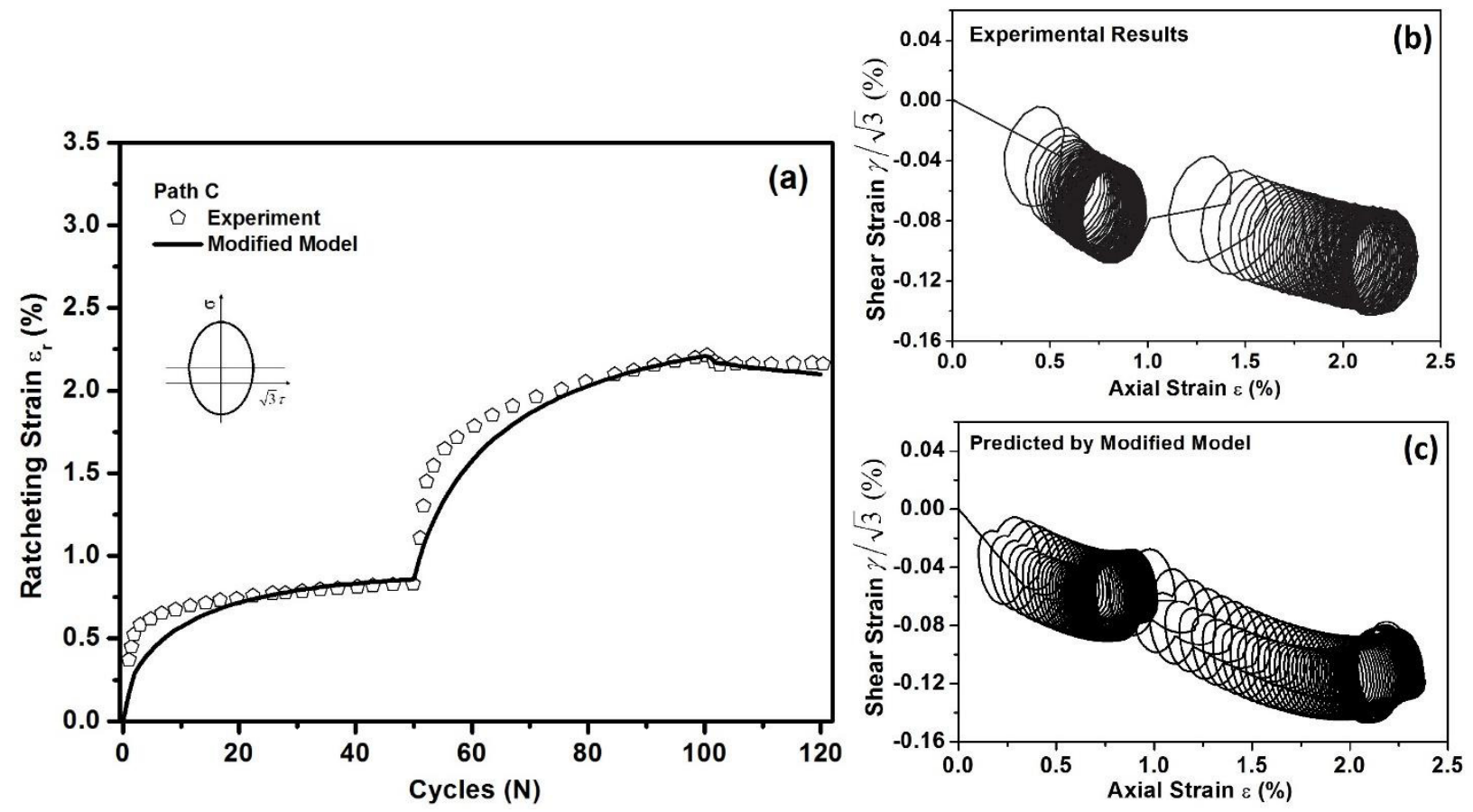

Figure 4.19 (a) Experimental ratcheting data [50] vs predicted ratcheting curves based on the modified model, (b) experimental hysteresis loops [43] ( $\varepsilon-\gamma)$, and (c) predicted hysteresis loops $(\varepsilon-\gamma)$ over load steps for history $\mathrm{C}$.

The experimental and predicted ratcheting values of 304 steel sample tested under history $\mathrm{D}$ is presented in figure 4.20a. The close agreement of the predicted ratcheting curves and experimental data over steps 1 and 3 is evident. The predicted curve in the middle step showed an underestimation of ratcheting not exceeding $8 \%$ as compared with experimental data. Over loading steps of history D shear stress amplitudes remained constant $\tau_{\mathrm{a}}=143.2 \mathrm{MPa}$ over loading steps. The experimental and predicted hysteresis loops $(\varepsilon-\gamma)$ in figures $4.20 \mathrm{~b}$ and $4.20 \mathrm{c}$ showed close agreements in both trend and magnitude of axial and shear strain values over hysteresis cycles. 

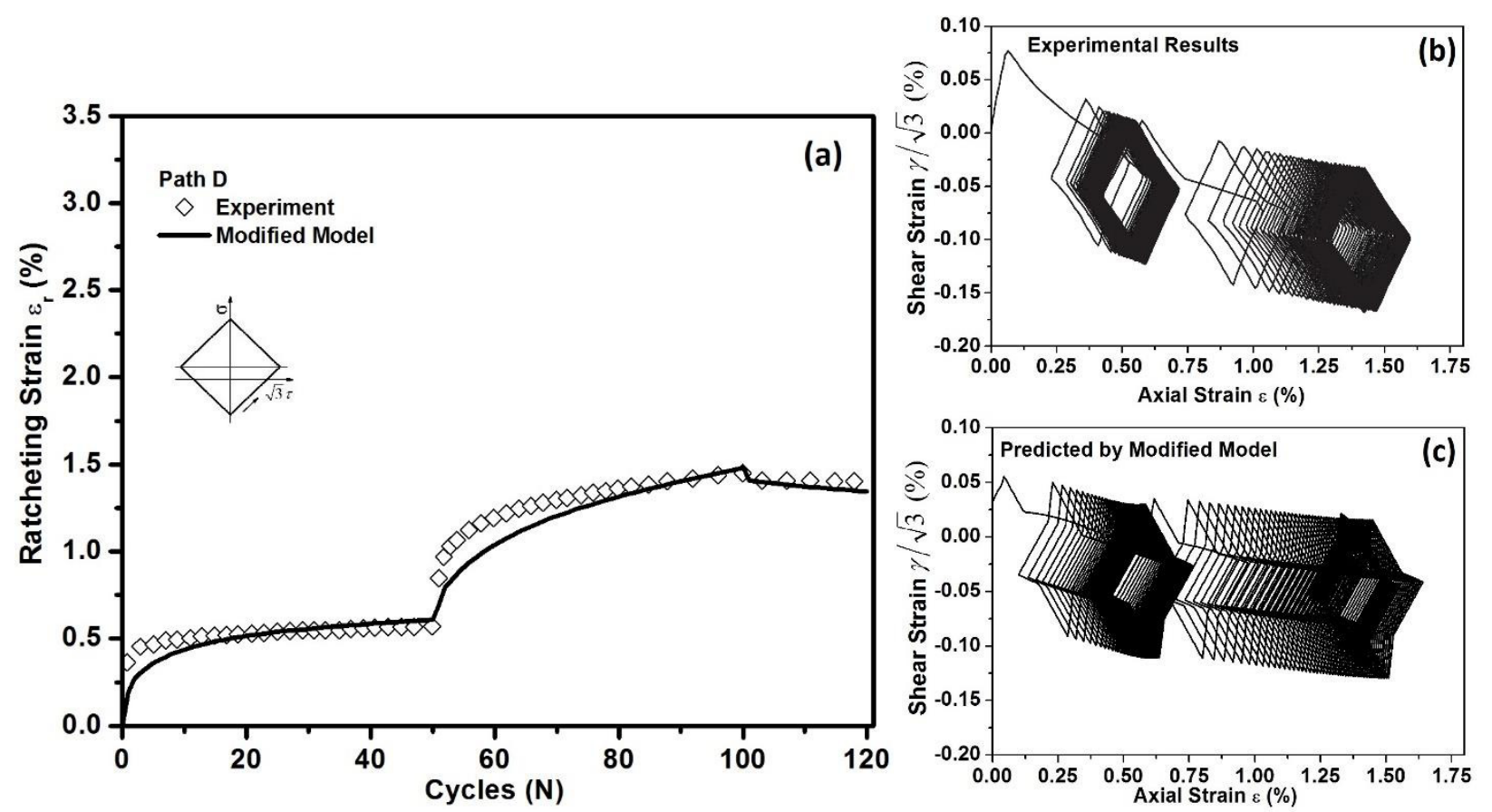

Figure 4.20 (a) Experimental ratcheting data [43] vs predicted ratcheting curves based on the modified model, (b) experimental hysteresis loops [50] ( $\varepsilon-\gamma)$, and (c) predicted hysteresis loops $(\varepsilon-\gamma)$ over load steps for history D.

Figure 4.21a compares the predicted ratcheting curves with the experimental ratcheting data for diamond loading path $\mathrm{E}$. The predicted ratcheting curve underestimated experimental data half-way through step 1 cycles. The predicted ratcheting values agreed with experimental data within the third loading step. The predicted curve fell below ratcheting data at $7 \%$ over the second loading step.

Path E is similar to path D over load steps of axial stress cycles. The diamond loading path E however possessed different shear stress amplitudes over load steps. The shear stress amplitude was kept constant $\tau_{\mathrm{a}}=71.6 \mathrm{MPa}$ for history $\mathrm{E}$. The higher shear stress amplitude of history $\mathrm{D}$ $\left(\tau_{\mathrm{a}}=143.2 \mathrm{MPa}\right)$, resulted in a smaller axial strain up to $1.45 \%$ as compared with that of $2.4 \%$ for loading path E. The experimental and predicted hysteresis loops $(\varepsilon-\gamma)$ in figures $4.21 \mathrm{~b}$ and $4.21 \mathrm{c}$ showed close agreements in both trend and magnitude of axial-shear strain hysteresis loops. The 
lower shear stress amplitude for history E dropped the range of shear strain as compared with that of history D with higher shear stress amplitude over loading steps.
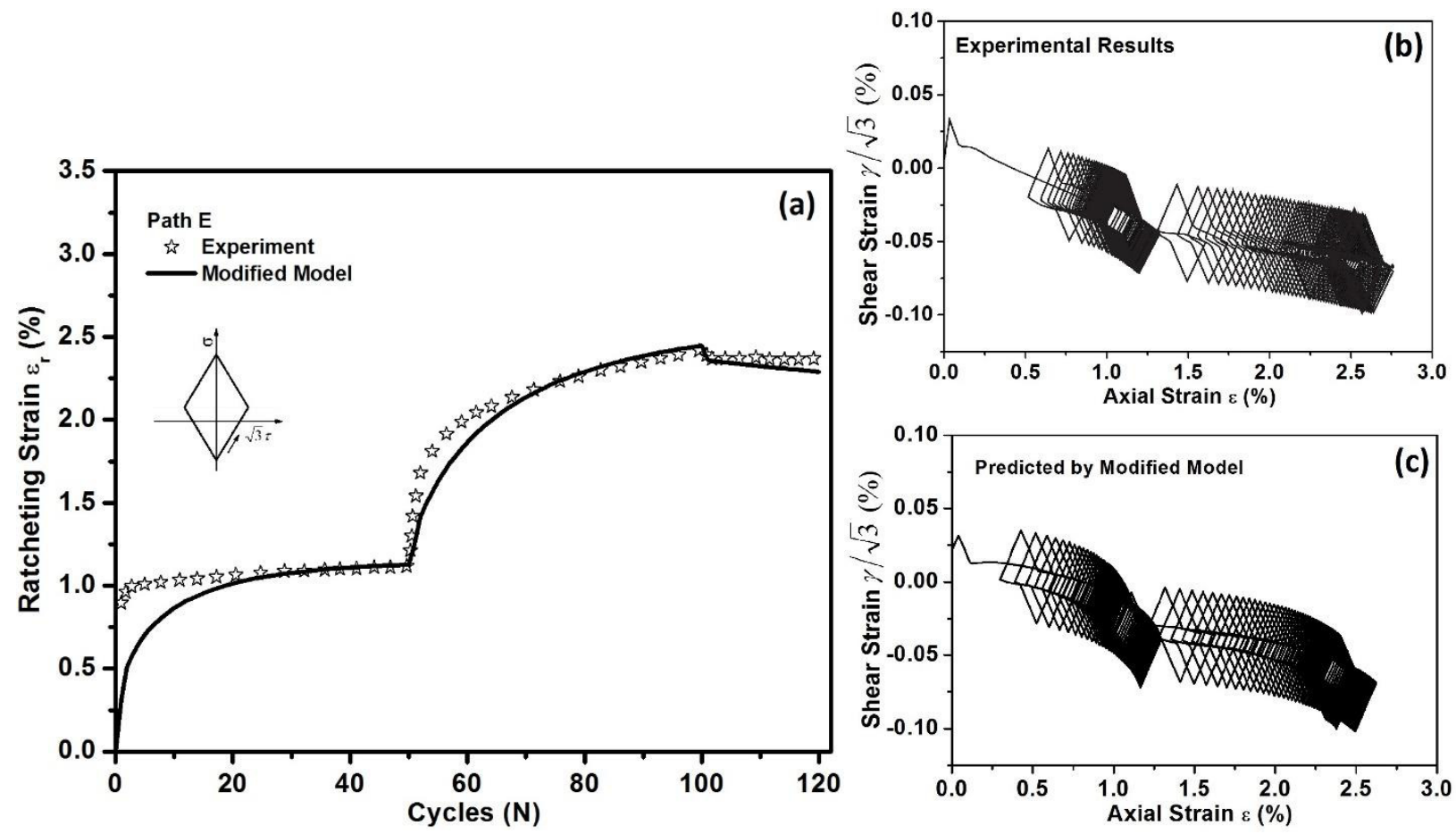

Figure 4.21 (a) Experimental ratcheting data [50] vs predicted ratcheting curves based on the modified model, (b) experimental hysteresis loops [43] ( $\varepsilon-\gamma)$, and (c) predicted hysteresis loops $(\varepsilon-\gamma)$ over load steps for history E.

An increase in axial mean stress resulted in a buildup in ratcheting data of 304 stainless steel samples tested with low-high-low axial mean stress sequence in histories A-E as presented in figures $4.17 \mathrm{a}-4.21 \mathrm{a}$. The change in direction of ratcheting over the third load step lowering the ratcheting magnitude is evident from these figures as the axial mean stress dropped at this step.

The buildup at the transient of step 1-step 2 and small peak at the transient points from step 2 to step 3 in these figures show how influential the loading sequence and drop in mean stress magnitude on the ratcheting response of 304 steel samples under step-loading conditions is.

The predicted ratcheting curves based on the modified hardening rule for loading path AE agreed with experimental data over load step 1 and particularly after a few initial cycles at this step as well as those experimental data within step 3. The predicted ratcheting data over middle 
load step however showed an underestimation as compared with experimental data for histories A-E. This underestimation has also been reported by Kang et al. [43] for loading paths B-E.

In figures 4.17-4.21, a drop in axial mean stress level in step 3 resulted in a suppression of the axial ratcheting strain values over this step. The shear ratcheting strain in figures $4.17 \mathrm{~b}$ and $4.17 \mathrm{c}$ was due to non-zero shear mean stress of $67.55 \mathrm{MPa}$ over loading steps of history A. The minor presence of shear ratcheting strain was evident in history B (figures $4.18 \mathrm{~b}$ and $4.18 \mathrm{c}$ ) at which shear mean stresses were absent over loading steps. In histories D and E, the difference in shear stress amplitudes noticeably affected the magnitude of axial ratcheting strain. Diamond loading path D resulted the ratcheting strain of $1.45 \%$ (see figures $4.20 \mathrm{~b}$ and $4.20 \mathrm{c}$ ) and loading path $\mathrm{E}$ resulted in a higher magnitude of axial ratcheting strain of $2.4 \%$ (see figures $4.21 \mathrm{~b}$ and 4.21c). The corresponding shear strain ranges in figures $4.20 \mathrm{~b}$ and $4.21 \mathrm{~b}$ over loading steps $1-3$ were found to be respectively $0.11 \%$ and $0.065 \%$.

\subsubsection{Multiaxial ratcheting of SS304 based on the O-W, C-J-K and modified A-V hardening rules under step-loading conditions}

The multiaxial ratcheting response of 304 stainless steel samples under various steploading histories A-E were predicted by means of the $\mathrm{O}-\mathrm{W}, \mathrm{C}-\mathrm{J}-\mathrm{K}$ and modified A-V kinematic hardening rules. The experimental ratcheting data were presented with those of predicted based on the modified model (Eq. (3.8)), O-W (Eq. (2.8)) and C-J-K (Eq. (2.13)) hardening rules in figure 4.22. In this figure, histories A-E possessed low-high-low sequences in mean stresses while stress amplitude stayed unchanged over load steps. This increase in the mean stress magnitude at the second loading step shifted up ratcheting strain values at this stage resulting in a peak at the transition of load steps 2 and 3 and subsequently as the mean stress in stage 3 decreased, the direction of ratcheting accumulation was reversed.

Predicted ratcheting curves based on the modified model in figures $4.22 \mathrm{a}-4.22 \mathrm{e}$ consistently agreed with those of experimentally obtained under multiaxial loading paths A-E 
beyond first few cycles of the first step as well as entire step 3. The predicted curves over step 2 slightly underestimated ratcheting for these histories over the second step.

The proposed model through introducing new terms including $\left\langle d \bar{\varepsilon}_{p} \cdot \bar{a} /|\bar{a}|\right\rangle,(2-\bar{n} \cdot \bar{a} /|\bar{a}|)$ and $\langle\bar{n} . \bar{a} /|\bar{a}|\rangle^{1 / 2}$, and calibrating coefficient $\gamma_{2}$ in its dynamic recovery term was capable to closely agree the trend of experimental ratcheting data over the multiaxial step-loading histories. The replacement of $d p$ with $\left\langle d \bar{\varepsilon}_{p} \cdot \bar{a}|| \bar{a} \mid\right\rangle$ further improved the capability of model to assess multiaxial ratcheting strain as the function in the MaCaulay brackets distinguishes different directions of $d \bar{\varepsilon}_{p}$ and $\bar{a} /|\bar{a}|$ under multiaxial stress cycles. Term $(2-\bar{n} . \bar{a} /|\bar{a}|)$ taking values between 1 and 3 under multiaxial loading conditions, magnifies the calibrating coefficient $\gamma_{2}$ and shifted down the ratcheting strain predicted by the modified model. Term $(\bar{n} \cdot \bar{a} /|\bar{a}|)$ taking values between -1 and 1 produces positive values smaller than 1, as it was employed in MaCaulay brackets in the form of $\langle\bar{n} . \bar{a} /|\bar{a}|\rangle^{1 / 2}$. This term controls the accumulation of ratcheting strain over stage II and prevents the modified model trapped in plastic shakedown.

The ratcheting curves were predicted based on the O-W and C-J-K hardening rules considering eight backstress components $M=8$. For loading paths A-D, the $\mathrm{O}-\mathrm{W}$ model overestimated ratcheting response of 304 stainless steel samples in figure 4.22 within the first step. The O-W model persistently overestimated the ratcheting strain of SS304 over subsequent steps 2 and 3 under paths $\mathrm{B}$ and $\mathrm{D}$. The overestimation of multiaxial ratcheting based on the $\mathrm{O}-\mathrm{W}$ model was consistently reported by Chen and Jiao [29] for cyclically hardened steel samples. This overestimation was earlier attributed to the absence of non-proportionality factor to control the evolution of ratcheting over complex stress cycles [36]. 

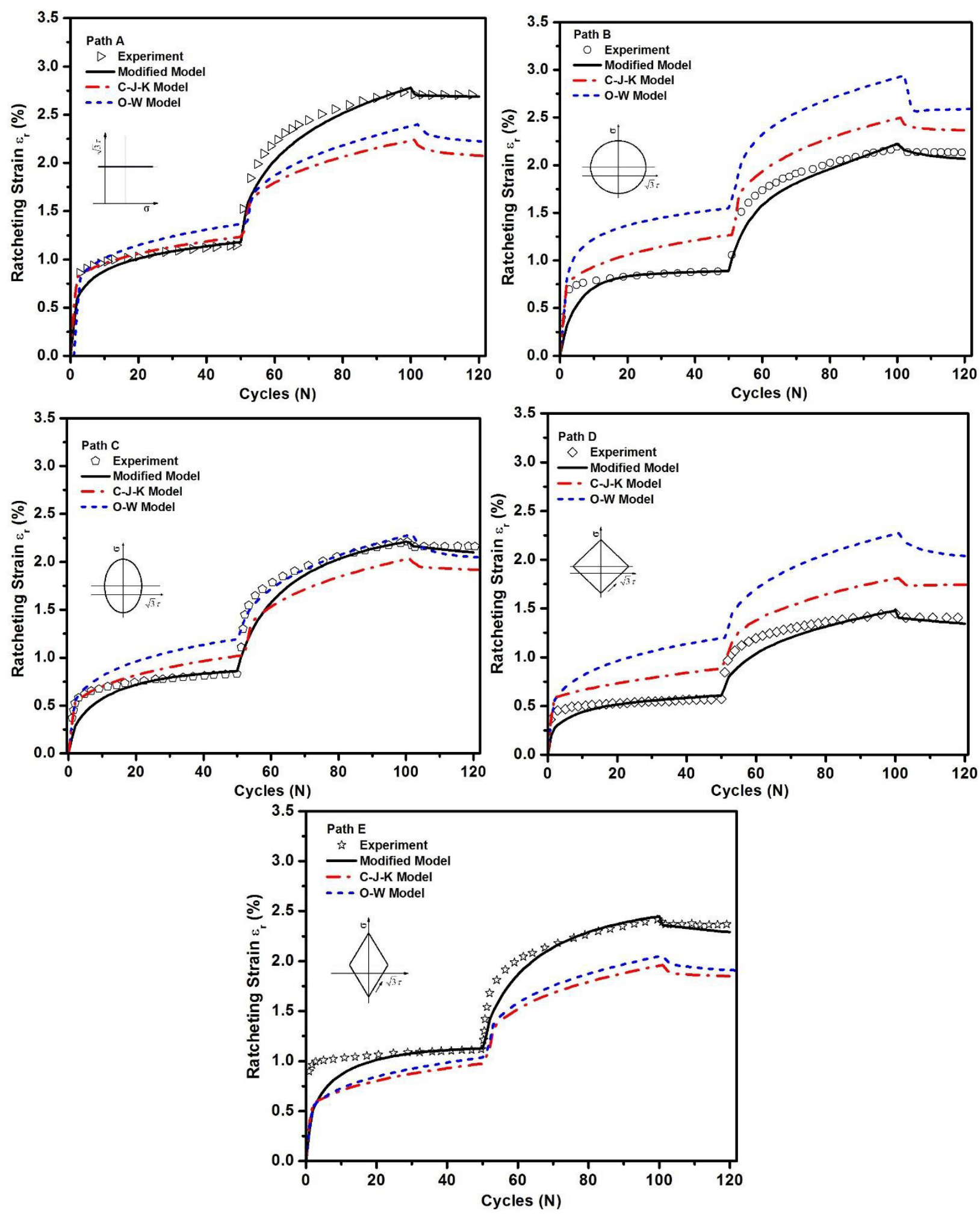

Figure 4.22 Experimental data [50] and predicted ratcheting strain values of 304 stainless steel samples based on the O-W, C-J-K and A-V modified hardening rules under various loading histories A-E. 
In the present study, the predicted ratcheting curves under loading history A resulted in a slight overestimation by the O-W model over the first loading step followed by underestimations of the second and third loading steps. The predicted curves through the O-W model showed a closer agreement with experimental results in steps 2 and 3 after the overestimation of the first step under loading history $\mathrm{C}$. The hardening rule of $\mathrm{O}-\mathrm{W}$ showed a persistent underestimation over load steps in loading history E. This model enabled to assess ratcheting in opposite direction as mean stress magnitude followed high-low sequence within steps 2 and 3 of loading histories A-E.

Constants $m_{i}, r_{i}$ and $\gamma_{i}$ in the kinematic hardening rules of O-W $[19,20]$ and C-J-K [30] are defined by segments chosen on the uniaxial stress-strain and uniaxial ratcheting curves through a tedious and time-consuming trial procedure. The deviation of predicted ratcheting curves based on the $\mathrm{O}-\mathrm{W}$ model in figures 4.22 was associated to the absence of the non-proportionality factor in the framework of the O-W. The C-J-K model addressed this shortcoming of the O-W model by incorporating term $\left\langle\bar{n} .\left(\bar{a}_{i} /\left|\bar{a}_{i}\right|\right)\right\rangle^{\chi_{i}}$ into the dynamic recovery term to include the effect of loading non-proportionality. Term $\left\langle\bar{n} . \bar{a}_{i} /\left|\bar{a}_{i}\right|\right\rangle$ takes different values of either zero or a value less than unity under non-proportional loading and the terms in the MaCaulay brackets $\left\langle\bar{n} \cdot\left(\bar{a}_{i} /\left|\bar{a}_{i}\right|\right)\right\rangle^{x_{i}}$ encounter the loading non-proportionality in ratcheting assessment of materials for exponent $\chi_{i}>0$.

The exponent $\chi_{i}$ in this model was first calibrated based on a set of multiaxial ratcheting data and then was employed for various loading paths. For 304 stainless steel samples discussed in this study, exponent $\chi_{i}=1.3$ was initially calibrated to coincide the ratcheting curve predicted by the C-J-K model with those of experimentally obtained under loading Path A. This value was then employed to predict the ratcheting under stress-controlled paths B-E. For loading paths at which the O-W model showed overproduction, the predicted ratcheting curves by the C-J-K model fell closer to experimental data as compared with those predicted curves by the O-W model mainly due to implementing term $\left\langle\bar{n} .\left(\bar{a}_{i} /\left|\bar{a}_{i}\right|\right)\right\rangle^{\chi_{i}}$. For the loading histories B and D at which the O-W model overestimated the ratcheting curves, the C-J-K hardening rule resulted in shifting down the 
ratcheting curves and closer agreements with the experimental data. The predicted ratcheting curves by the C-J-K model further shifted down the ratcheting curves as the predicted curves of A, C, and E histories through the $\mathrm{O}-\mathrm{W}$ rule fell below experimental data. The C-J-K model showed more deviation of the predicted curves as compared with experimental ratcheting data over loading paths $\mathrm{A}$ and $\mathrm{E}$ as compared to those predicted by the $\mathrm{O}-\mathrm{W}$ model.

The C-J-K model with the inclusion of multiaxial factor $\chi_{i}$ showed the capability to follow the trend of ratcheting strain under non-proportional step-loading conditions and to accumulate the ratcheting strain in the opposite direction of axial mean stress at step 3 consistent with experimental data as shown in figure 4.22 .

In the transient stage form the second step to the third step the ratcheting response of SS304 showed a small peak predicted by the O-W, C-J-K and modified A-V hardening rules. These small peaks formed in the transient stage from step 2 to step 3 verifying how influential the effect of the loading sequence in ratcheting response under step-loading histories is. Kang et al. also [52] reported that under a step-loading history each step affected the ratcheting progress over subsequent load steps depending on the sequence of loading steps.

The $\mathrm{O}-\mathrm{W}$ and $\mathrm{C}-\mathrm{J}-\mathrm{K}$ predictions were significantly influenced by the number of backstress components, $M$, exponent $m_{i}$ and parameters $r_{i}$ and $\gamma_{i}$ defined by segments chosen on the uniaxial stress-strain curve through a tedious and time-consuming trial procedure. Both the C-J-K and modified models took into account the effect of non-proportionality. The C-J-K rule improved the overestimated ratcheting curves predicted by the $\mathrm{O}-\mathrm{W}$ model as a non-proportionality factor was introduced into the dynamic recovery term.

Experimental ratcheting data of 304 steel samples under multiaxial step-loading conditions showed great influence of the prior load step history on the ratcheting response over the subsequent loading steps. The material showed slight ratcheting accumulation in the opposite direction of axial mean stress. The high-low axial mean stress load sequence in the transient stage of step 2 and step 3 resulted in the restraining of the ratcheting progress. The difference in ratcheting magnitudes 
over loading steps 1 and 3 for histories A-E at which both steps possess identical applied stress magnitudes can also be attributed to the influence of loading sequence involving the prior load step with higher axial mean stress. This is believed [73] to improve materials strength and to decrease the rate of ratcheting progress in the subsequent step.

\subsection{Summary}

The A-V hardening rule is modified through its linear hardening and dynamic recovery term. The linear term of backstress holds material dependent coefficients of $C$ and $\gamma_{l}$ controlling shape and size of hysteresis loops while the dynamic recovery term possesses calibrating coefficient $\gamma_{2}$ to control the ratcheting strain rate over stress cycles. Materials dependent coefficients $\mathrm{C}, \gamma_{1}, \gamma_{2}$ are determined based on uniaxial ratcheting data.

The modified rule through the involvement of $d \bar{\varepsilon}_{p}$ and $\bar{a} /|\bar{a}|$ in the MaCaulay brackets $\left\langle d \bar{\varepsilon}_{p} \cdot \bar{a} /|\bar{a}|\right\rangle$ is capable to track different directions under multiaxial loading. This function further decreased the overall ratcheting curve and provided a closer predicted curves to the experimental data. The non-proportionality term $(\bar{n} \cdot \bar{a} /|\bar{a}|)$ is introduced in the form of multipliers $(2-\bar{n} \cdot \bar{a} /|\bar{a}|)$ and $\langle\bar{n} \cdot \bar{a} /|\bar{a}|\rangle^{1 / 2}$ to respectively regulate dynamic recovery components $\gamma_{2}$ and $\bar{b}$ under multiaxial loading. Term $(2-\bar{n} . \bar{a} /|\bar{a}|)$ further shifted down the predicted ratcheting strain, while term $\langle\bar{n} . \bar{a} /|\bar{a}|\rangle^{1 / 2}$ prevented modified model to predict plastic shakedown over stage II.

The modified hardening rule was employed to predict ratcheting response of 1045 steel samples for eight different multiaxial loading paths A-H. The predicted curves closely agreed with experimental data over ratcheting stages I and II. Ratcheting response of 1045 and $1 \mathrm{Cr} 18 \mathrm{Ni}$ Ti steel samples undergoing multiaxial loading cycles was assessed based on nonlinear hardening rules of the $\mathrm{O}-\mathrm{W}, \mathrm{J}-\mathrm{S}, \mathrm{McD}$ owell and modified A-V. The O-W, J-S, McDowell hardening rules with relatively complex structure and several coefficients were compared with the modified model 
with less complexity and number of coefficients. The O-W, J-S and McDowell models overestimated the ratcheting strain of 1045 and $1 \mathrm{Cr} 18 \mathrm{Ni}$ Ti steel samples for various loading histories, while the predicted curves of the modified model closely agreed with experimental data of 1045 and $1 \mathrm{Cr} 18 \mathrm{Ni9Ti}$ steel samples over ratcheting stages I and II. The J-S and McDowell hardening rules developed the $\mathrm{O}-\mathrm{W}$ rule to include the effects of stress-dependency and nonproportionality in ratcheting assessment of materials through modifications over exponent $m_{i}$. The predicted ratcheting curves by the McDowell and J-S models highly overestimated the ratcheting response of steel samples under various multiaxial loading paths as compared with the experimental data. The J-S model has shown slightly lower overestimation than that of the McDowell model mainly due to its $m_{i}$ formulation. The O-W, J-S and McDowell models possessed more complicated structures.

Ratcheting response of 304 stainless steel samples undergoing stress-controlled and combined stress- and strain-controlled multiaxial tests was assessed based on hardening rules of the O-W, C-J-K and modified model. The O-W model overestimated the ratcheting strain of SS304 samples for various loading histories. The C-J-K model holding a non-proportionality term resulted in closer agreement of the predicted ratcheting curves and experimental data for loading paths A3, A4, B1, and B4. The modified model closely agreed with experimental data of 304 stainless steel samples over ratcheting stages I and II with some deviation over initial stress cycles. Ratcheting response of 304 stainless steel samples undergoing multiaxial step-loading tests was assessed based on nonlinear hardening rules of the O-W, C-J-K and modified model. Load sequence greatly affected the ratcheting strain rate and its trend over the steps. Low-high-low axial mean stress sequence studied in this paper resulted in a buildup of ratcheting strain values from the first step to the second step, followed by restraining and changing the direction of ratcheting data over the third loading step. The predicted ratcheting curves through the modified hardening rule showed a close agreement as compared with the ratcheting experimental data over multiaxial load steps in 304 steel samples undergoing various loading histories A-E. The O-W model closely predicted the ratcheting strain of 304 steel samples under loading path $\mathrm{C}$. The predicted curves 
through this model deviated from experimental data for histories A, B, D, and E. The C-J-K model through its non-proportionality term resulted in a closer agreement of the predicted ratcheting curves and experimental data for the loading paths B and D overestimated through the O-W model. The C-J-K model exacerbates the ratcheting prediction of the $\mathrm{O}-\mathrm{W}$ model for loading histories A, $\mathrm{C}$, and E. The O-W, C-J-K and modified A-V hardening rules enabled ratcheting prediction over step 3 at which mean stress reduced resulting in a change in the ratcheting direction over this loading step. The O-W, J-S, McDowell and C-J-K models possessed more complicated structures. The prediction of ratcheting curves based on these hardening rules with more number of backstress components, and with larger exponent $m_{i}$ required longer CPU time to run the models while time required to predict ratcheting over stress cycles by the modified model was twice shorter due to less complicated hardening rule and limited number of coefficients. 


\section{CHAPTER FIVE}

\section{CONCLUSIONS AND RECOMMENDATIONS}

\subsection{Conclusions}

The proposed kinematic hardening rule in this study offers a simple framework with three material dependent coefficients $C, \gamma_{1}$ and $\gamma_{2}$, to predict the ratcheting strain developed over multiaxial loading cycles mainly due to modifications in the dynamic recovery term. In the dynamic recovery of the modified hardening rule, the term $\left\langle d \bar{\varepsilon}_{p} \cdot \bar{a} /|\bar{a}|\right\rangle$ was replaced with the accumulated plastic strain increment, $d p$. The term $\left\langle d \bar{\varepsilon}_{p} \cdot \bar{a} /|\bar{a}|\right\rangle$ introduced a slower evolution of ratcheting strain in the deviatoric stress space as the components $d \bar{\varepsilon}_{p}$ and $\bar{a} /|\bar{a}|$ in the MaCaulay brackets took different directions under multiaxial loading conditions and made the model capable of tracking these different directions. The projection presented by this term resulted in a slower evolution of ratcheting under multiaxial loading compared to term $d p$. The measure of nonproportionality was also utilized in the form of product of $\langle\bar{n} \cdot \bar{a} /|\bar{a}|\rangle^{1 / 2}$ and vector $\bar{b}$ to control the accumulation rate of ratcheting strain, and essentially to prevent the modified model to experience plastic shakedown as the number of cycles advances over various multiaxial loading conditions. Under multiaxial loading histories, the term $\langle\bar{n} \cdot \bar{a} /|\bar{a}|\rangle^{1 / 2}$ takes positive values smaller than 1 and prevents this model from premature plastic shakedown. The term $(2-\bar{n} \cdot \bar{a} /|\bar{a}|)$ regulates the magnitude of coefficient $\gamma_{2}$ properly for various multiaxial loading paths. The coefficient $\gamma_{2}$ was determined using the ratcheting data under uniaxial loading through a trial-error procedure. This 
term takes the values between 1 and 3 under multiaxial loading and consequently magnifies the effect of $\gamma_{2}$ and results in a smaller accumulation rate of ratcheting strain over stress cycles. Term $(2-\bar{n} \cdot \bar{a} /|\bar{a}|)$ encounters the effect of non-proportionality as multiaxial loads are applied, and reduces to unity for uniaxial loading condition. The new modified hardening rule was employed to assess the ratcheting response of 1045 steel samples under various multiaxial loading paths. The applied modifications were individually studied to investigate the evolutional impact of introduced terms. The modified hardening rule offered a simple framework to successfully predict ratcheting strain over multiaxial loading cycles mainly due to its dynamic recovery terms with limited number of coefficients.

The multiaxial ratcheting strain of 1045 and $1 \mathrm{Cr} 18 \mathrm{Ni}$ Ti steel samples was assessed under various loading paths by means of the O-W, J-S, McDowell, and A-V modified hardening rules. The O-W, J-S, McDowell hardening rules with relatively complex structures and several coefficients were compared with the modified model with less complexity and number of coefficients. The O-W, J-S and McDowell models overestimated the ratcheting strain of 1045 and $1 \mathrm{Cr} 18 \mathrm{Ni} 9 \mathrm{Ti}$ steel samples under various multiaxial loading histories, while the predicted curves of the modified model closely agreed with experimental data of steel samples over all the ratcheting stages through terms $\left\langle d \bar{\varepsilon}_{p} \cdot \bar{a} /|\bar{a}|\right\rangle$ and $(\bar{n} . \bar{a} /|\bar{a}|)$ in the dynamic recovery, accounting for non-proportionality effect. The J-S and McDowell models have further developed the O-W model to introduce non-proportionality effects through the exponents $m_{i}$. The J-S model showed a closer agreement with experimental data due to the employed $m_{i}$ formulation. The McDowell presented a more overestimation as compared to the $\mathrm{O}-\mathrm{W}$ model.

The capability of the O-W, C-J-K and the modified hardening rule was investigated in multiaxial ratcheting assessment of SS304 steel samples under various stress-controlled and combined stress- and strain-controlled multiaxial paths. The O-W model persistently overestimated the ratcheting response of 304 stainless steel samples tested under various loading histories, while the predicted curves through the C-J-K model showed less overprediction for all 
cases and presented a close agreement with ratcheting experimental data for loading paths A3, A4, $\mathrm{B} 1$, and B4 due to the inclusion of non-proportionality term in its framework. The predicted ratcheting curves based on the modified model closely agreed with experimental data of 304 steel samples.

The ratcheting response of 304 stainless steel samples was also evaluated under three-step multiaxial loading histories by means of the modified hardening rule as well as the models of O$\mathrm{W}$ and C-J-K and the results were compared with experimentally obtained ratcheting data. The predicted ratcheting curves and generated axial-torsional hysteresis loops based on the modified model closely agreed with the experimentally obtained hysteresis loops of SS304 steel samples over the loading steps. The modified model was found capable of predicting ratcheting in the opposite direction as the tensile axial mean stress dropped from $117 \mathrm{MPa}$ to $78 \mathrm{MPa}$. The predicted ratcheting curve closely agreed with the experimental data over the first and third steps however a deviation of up to $8 \%$ between the predicted and experimental ratcheting values was evidenced within the second step.

The predicted ratcheting curves by the $\mathrm{O}-\mathrm{W}$ model showed deviations from the experimental data as compared with experimental ratcheting data of SS304 samples for histories $\mathrm{A}, \mathrm{B}, \mathrm{D}$, and $\mathrm{E}$, while presented a close agreement as the loading history $\mathrm{C}$ was applied. This deviation has been lowered than under loading conditions B and D predicted by the $\mathrm{O}-\mathrm{W}$ model as the C-J-K model holding non-proportionality term was employed. However, the C-J-K model aggravated the ratcheting prediction of the $\mathrm{O}-\mathrm{W}$ model for loading histories $\mathrm{A}, \mathrm{C}$, and $\mathrm{E}$.

The choice of hardening rule to assess ratcheting steel samples is found to be very much dependent on complexities involved with ratcheting algorithms, their constitutive equations and framework, coefficients, and CPU time required to run ratcheting programs. The $\mathrm{O}-\mathrm{W}, \mathrm{J}-\mathrm{S}$, McDowell and C-J-K models possessed more complicated structures with more number of backstress components as well as more number of coefficients, including $r_{i}, \gamma_{i}$ and $m_{i}$. The prediction of ratcheting strain on the basis of these complex framewoks required longer CPU time, 
while the time required to predict the ratcheting strain over loading cycles by the modified hardening rule was found more time-efficient due to requirement for less coefficients and less complicated framework.

\subsection{Recommendations for Future Research}

For reliable design of engineering components and structures undergoing asymmetric stress cycles, assessment of successive plastic strain referred as ratcheting strain in materials is always crucial. When both ratcheting and fatigue phenomena are coupled, they result in a severe damage leading to failure of components. Influential parameters and their interactions affecting ratcheting and fatigue phenomena need to be prioritized by the researchers in the field of cyclic plasticity and failure analysis, to more accurately investigate the effects of mechanistic parameters. The influencing parameters include non-proportionality under multiaxial loading conditions, microstructural characterization, cyclic softening/ hardening of materials, thermal stresses, and hostile environment.

Further research outlooks in the field of cyclic plasticity coupled damage is recommended for the reliable ratcheting and failure assessment of materials. Some of these plans are listed below:

* In addition to non-proportionality effects, influential parameters such as environmental and thermal conditions as well as rate-dependency need to be properly addressed as future research perspectives in the framework of hardening rules for a realistic ratcheting assessment of materials.

- Materials microstructures, dislocations characterizations and their evolution and interactions over stress cycles are prominent parameters in the ratcheting phenomenon and consequently introducing them in the modeling of ratcheting strain is recommended for future studies. 
* The proposed hardening rule was evaluated for steel alloys under a wide variety of stress- and stress/strain controlled multiaxial loading histories. Further investigations are recommended to assess the capability of the modified model in ratcheting prediction of other metallic, composites and smart materials.

In the kinematic hardening rules, it is assumed that the size and shape of yield surface stay unchanged during the plasticity over the deviatoric stress space. Combining the kinematic and isotropic hardening rules introduces change in the yield surface into the hardening rule. This can improve the capability of models in the ratcheting assessment of viscoplastic materials, and materials under very complex loading conditions which may be experiencing extra hardening.

The kinematic hardening rule of Ohno and Wang was defined on the basis of the critical state of the dynamic recovery term in the backstress equation. The total backstress in this hardening rule is defined based on the superposition of $M$ independent backstress components. It is recommended to employ the modified hardening rule to form $d \bar{a}=\sum_{i=1}^{M} d \bar{a}_{i} \quad$ superpositioning $M$ independent backstress components.

* The modified hardening rule in this study presented a promising results. This model has a simpler framework and requires less number of coefficients compared with the other models, these offer more robust analysis, consistency condition and consequently produces reliable component ratcheting prediction. Implementation of the developed model within a finite element code is recommended for ratcheting assessment of various geometry components. 


\section{APPENDIX A}

Appendix A presents MATLAB Programming code developed on the basis of the modified kinematic hardening rule in this thesis to assess ratcheting response of 1045, SS304 and 1Cr18Ni9Ti steel alloys under various multiaxial loading spectra. MATLAB Programming codes for the O-W, McDowell, J-S and C-J-K hardening rules were also developed as subroutine programs to assess multiaxial ratcheting under stress-controlled and mixed stress- and straincontrolled conditions. The codes are developed based on incremental theory of plasticity and include steps of analysis for various loading paths, stress/strain tensors, deviatoric stress components and yield surface translations. Table A.1 lists symbols and terms employed in the developed MATLAB programming codes.

Table A.1 Symbols and terms used in the MATLAB programming codes.

\begin{tabular}{|c|c|}
\hline $\mathrm{E}$ & Young's modulus of elasticity \\
\hline G & Shear modulus \\
\hline$n i$ & Poisson's ratio \\
\hline $\mathrm{n}$ & Normal vector yield surface \\
\hline a & Total backstress tensor \\
\hline $\mathrm{Da}$ & Increments of backstress tensor \\
\hline $\mathrm{ai}$ & Total backstress tensor of $\mathrm{i}^{\text {th }}$ components in $\mathrm{O}-\mathrm{W}$ \\
\hline Dsig & Increments of stress tensor \\
\hline Eps & Total strain \\
\hline DEps & Increments of total strain \\
\hline DEps_e & Increments of elastic strain \\
\hline DEps_p & Increments plastic tensor \\
\hline Ddev_Sig & Increments of deviatoric stress \\
\hline $\mathrm{Hp}$ & Modulus of plasticity \\
\hline $\mathrm{Db}$ & Increments of the second internal variable \\
\hline $\mathrm{Cb} 2$ & Coefficient $\gamma_{2}$ in the modified model \\
\hline $\mathrm{Cb} 1$ & Coefficient $\gamma_{1}$ in the modified model \\
\hline $\mathrm{A} \mathrm{b}$ & Coefficient $\mathrm{C}$ in the modified model \\
\hline A_Oi & Calibrating exponent of McDowell and J-S models \\
\hline $\mathrm{Bi}$ & Noncoaxial calibrating exponent of McDowell model \\
\hline $\mathrm{Xi}$ & Mutiaxiality factor of C-J-K model \\
\hline $\operatorname{aexx}$ & Ratcheting strain in axial direction \\
\hline aexy & Ratcheting strain in axial direction \\
\hline
\end{tabular}




\section{MATLAB Programs for the Modified Hardening Rule}

\section{Main program under mixed stress- strain-controlled loading conditions}

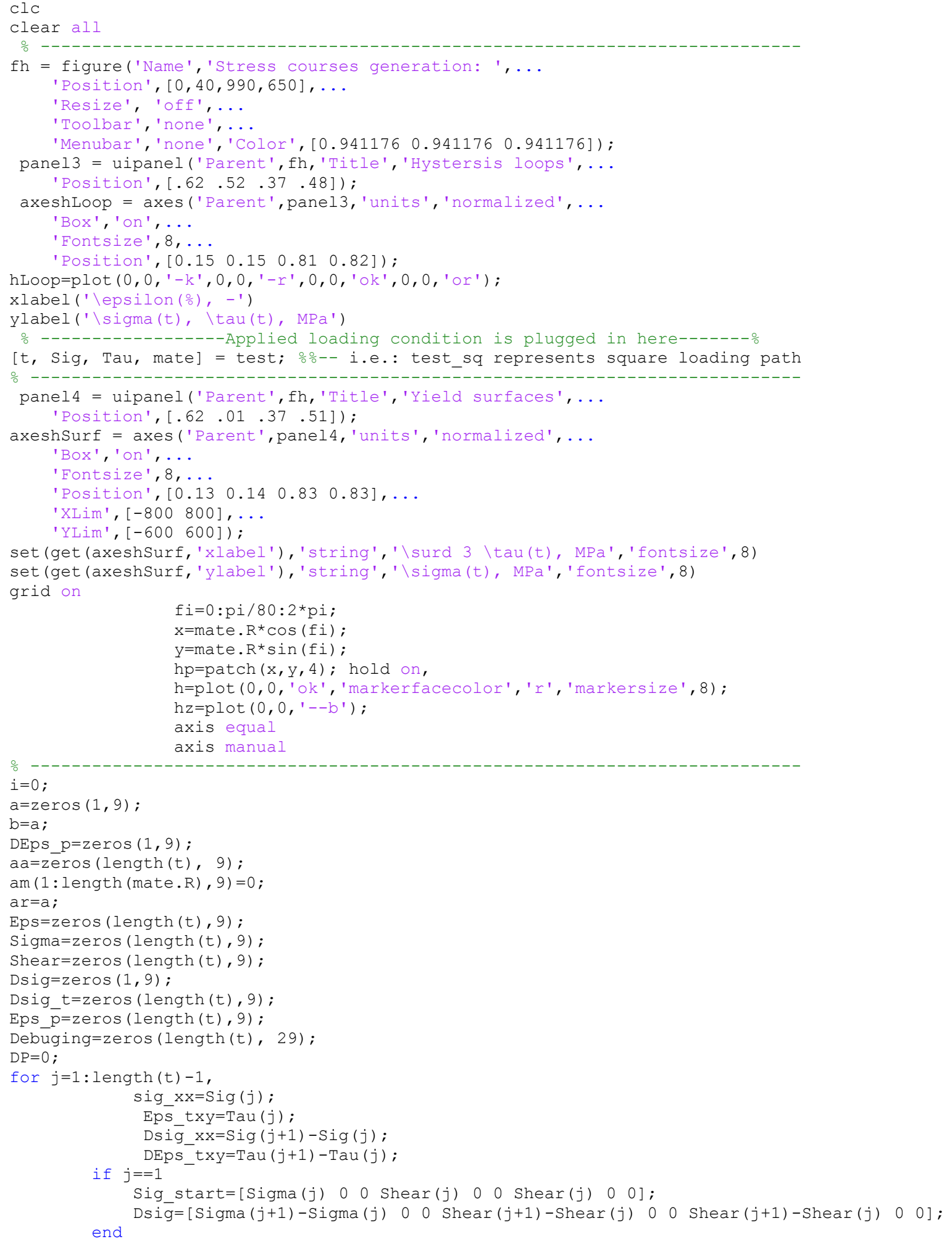


[Debuging, i, a, ar, DEps_p, b, ... DP,Dsig,DEps_t]=calculation(Sig_start, Dsig, i, a, mate,j,Debuging, ar, DEps_p, b, DP,Dsig_xx,DEps_txy);

$\operatorname{Sigma}(j+1,:)=\operatorname{Sigma}(j,:)+\operatorname{Dsig} ;$

Dsig_t $(j,:)=$ Dsig;

Sig_start $=$ Sigma $(j+1$

$\operatorname{Eps}(j+1,:)=\operatorname{Eps}(j,:)+\operatorname{DEps} t$

Eps_p $(j+1,:)=E p s \_p(j,:)+\bar{D} E p s \_p ;$

$\mathrm{aa}(j+1,:)=\mathrm{a}$;

$\%$ (1, :

set (hLoop (1), 'xdata', Eps $(1: j+1,1) * 100, '$ ydata', $\operatorname{Sigma}(1: j+1,1)$ )

set (hLoop (2), 'xdata', $\operatorname{Eps}(1: j+1,4) * 100, ' y d a t a ', \operatorname{Sigma}(1: j+1,4))$

set (hLoop (3), 'xdata', Eps $(j+1,1) \star 100$, 'ydata' , Sigma $(j+1,1))$

set (hLoop (4), 'xdata', Eps $(j+1,4) \star 100$, 'ydata', $\operatorname{Sigma}(j+1,4))$

drawnow expose

응

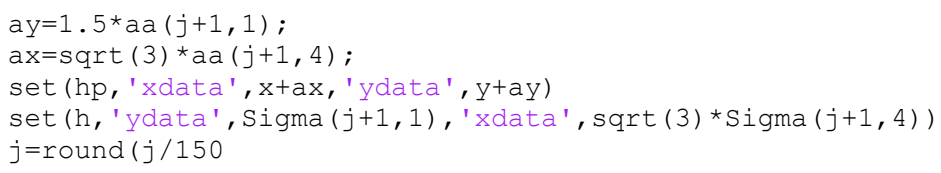

end

$\mathrm{k}=1$;

$\mathrm{z}=1$;

응음----------Ratcheting Calculation------------------ $\% \circ \%$

for $j=1:$ length $(t)$

if $\operatorname{rem}(t(j), .05)==0$

$\operatorname{Mexx}(z, 1)=\max (\operatorname{Eps}(k: j, 1)) ;$

$\operatorname{Nexx}(z, 1)=\min (\operatorname{Eps}(k: j, 1)) ;$

$\operatorname{aexx}(z, 1)=(\operatorname{Mexx}(z, 1)+\operatorname{Nexx}(z, 1)) \star 100 / 2$;

$\operatorname{Mexy}(z, 1)=\max (\operatorname{Eps}(k: j, 4)) ;$

$\operatorname{Nexy}(z, 1)=\min (\operatorname{Eps}(k: j, 4))$;

$\operatorname{aexy}(z, 1)=(\operatorname{Mexy}(z, 1)+\operatorname{Nexy}(z, 1)) \star 100 / 2$;

$\mathrm{k}=\mathrm{j}+1$

$\mathrm{z}=\mathrm{z}+1$

end

end 


\section{Calculation subroutine for modified model under mixed stress- strain-controlled loading conditions}

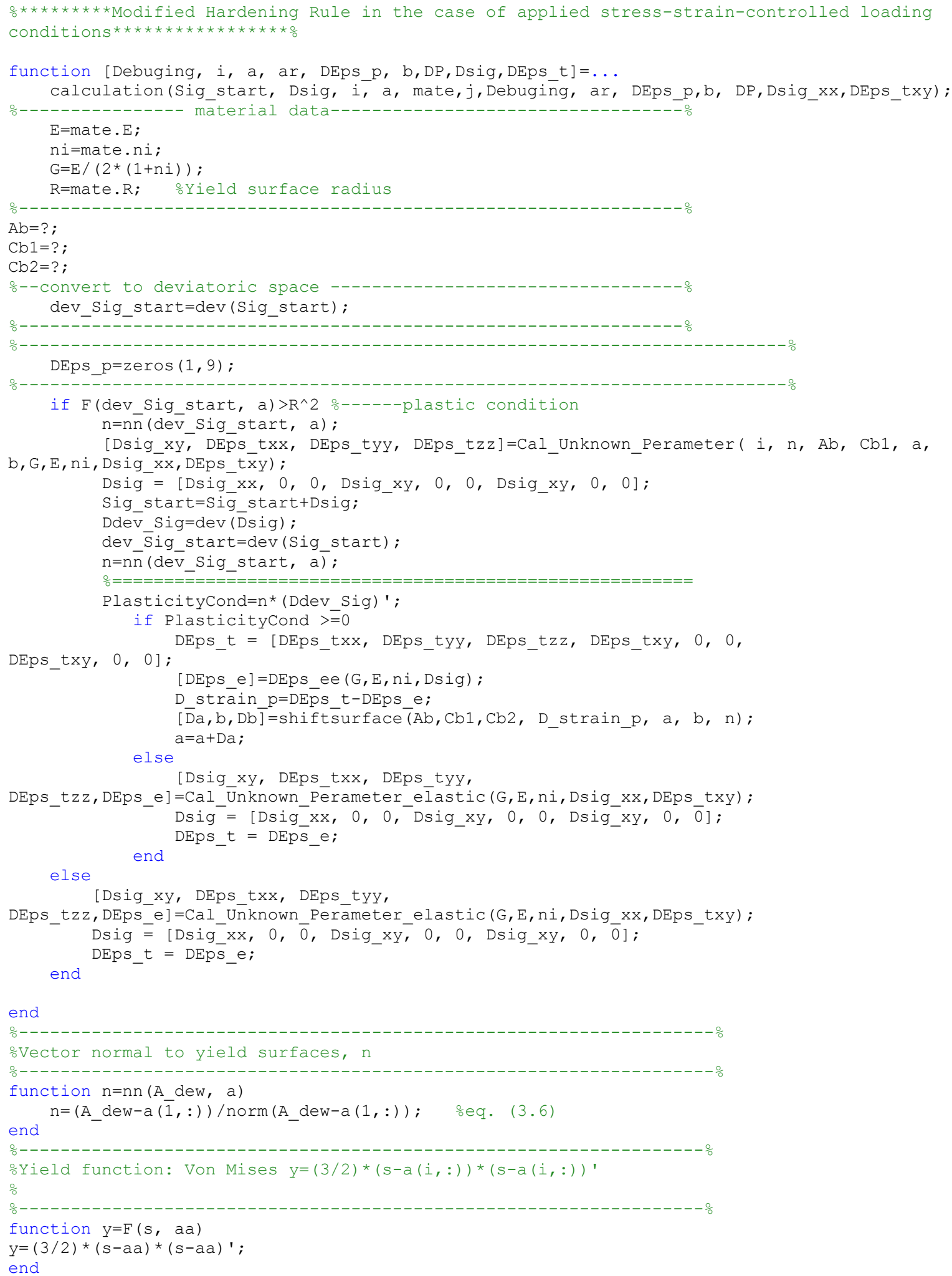




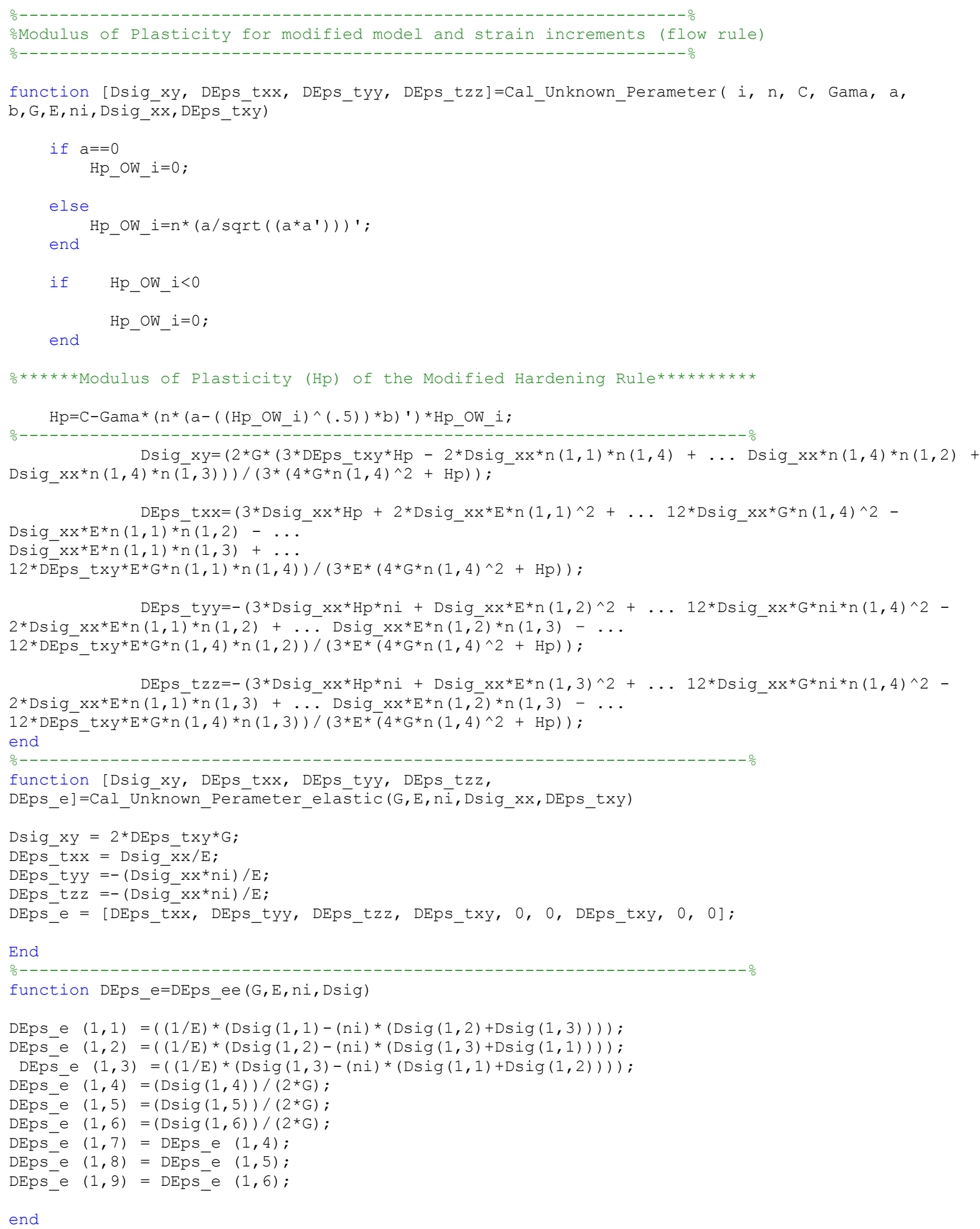




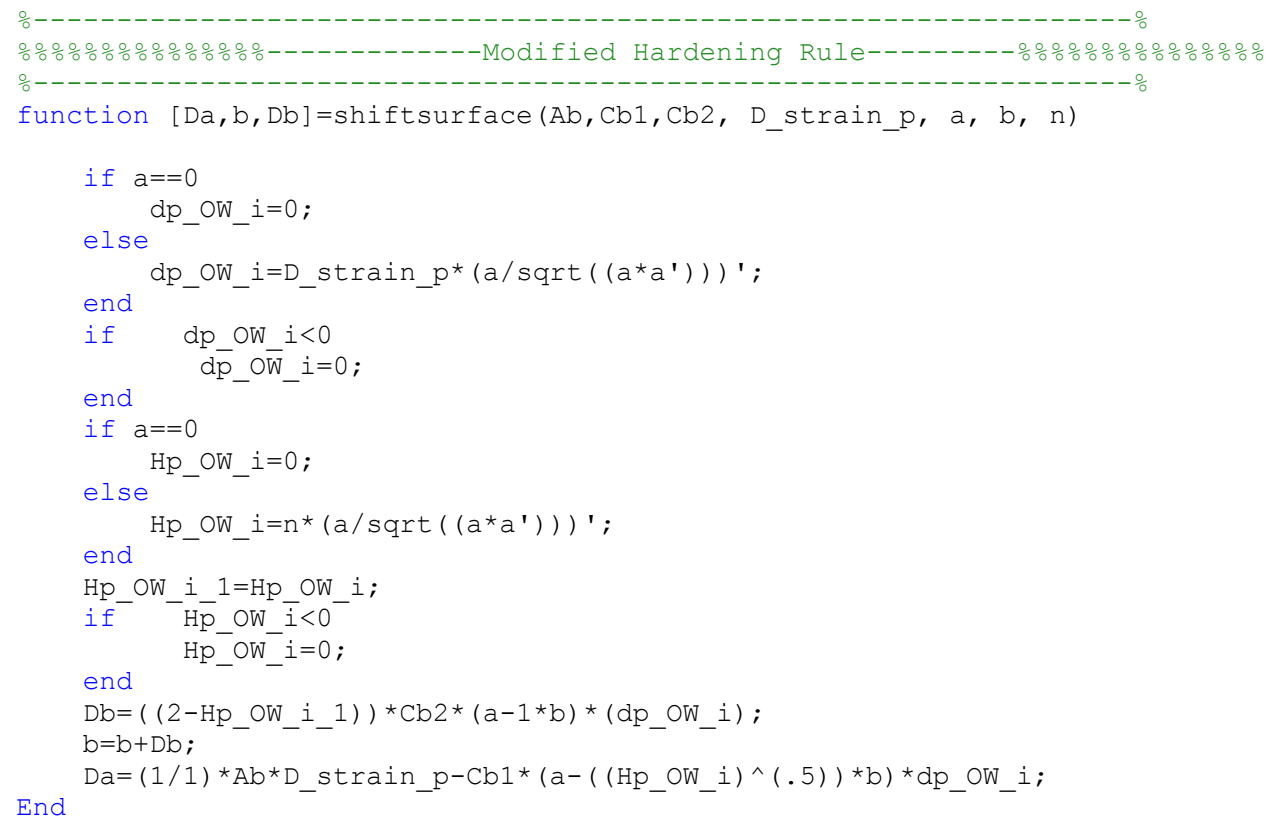




\section{Deviatoric stress subroutine ${ }^{1}$}

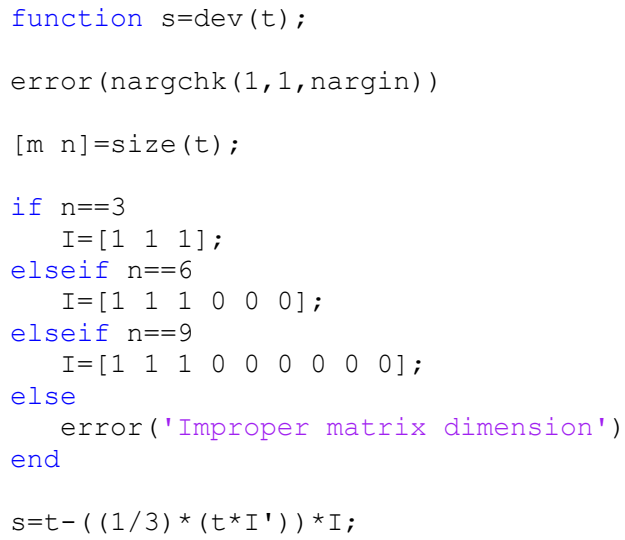

\section{Material properties subroutine ${ }^{2}$}

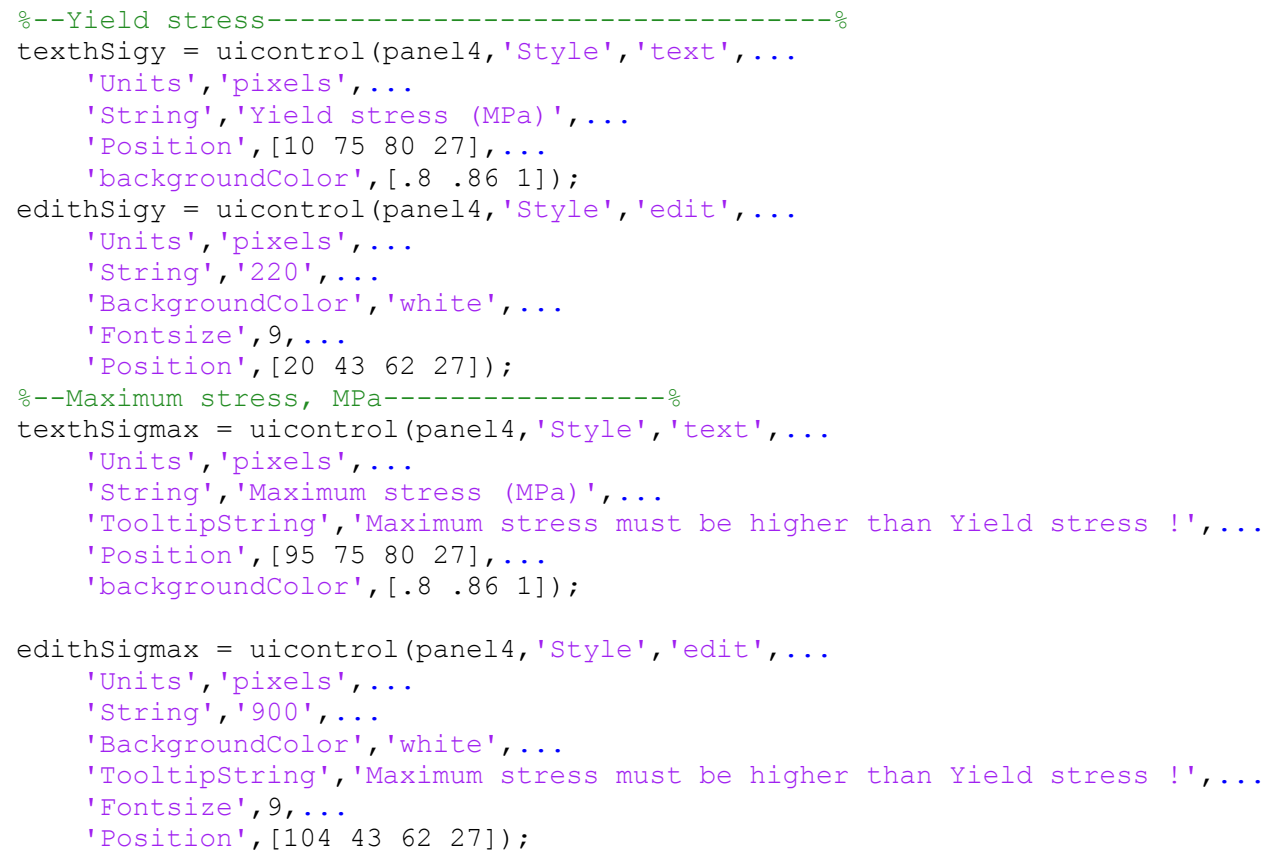

${ }^{1}$ Material properties subroutine was taken from [74]

2 Hooke's Law subroutine was taken from [74] 


\section{Stress generation subroutine under mixed stress- strain-controlled loading conditions 3}

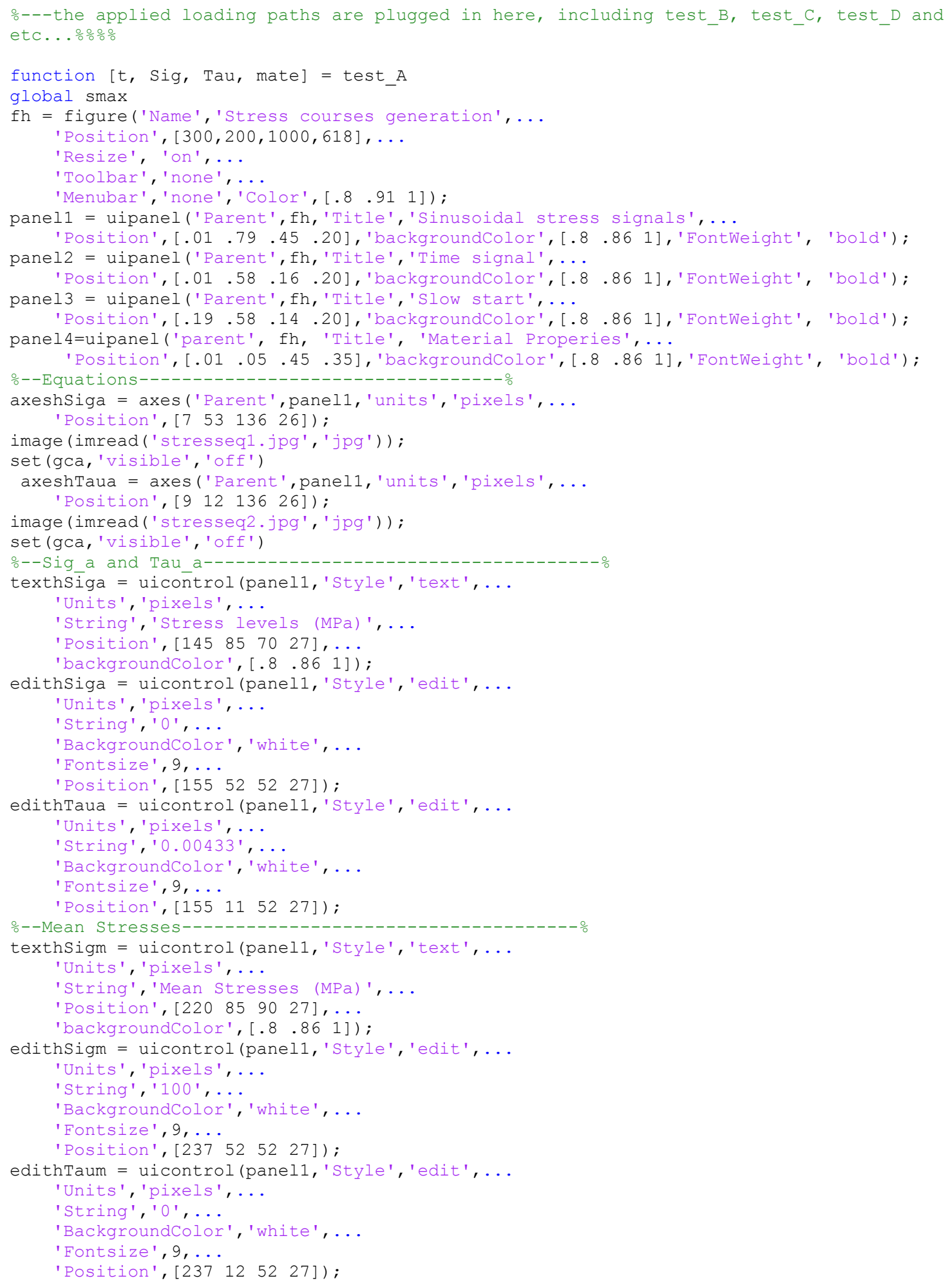

${ }^{3}$ The graphical programming in stress generation subroutine pages 105-107 was taken from [74] 


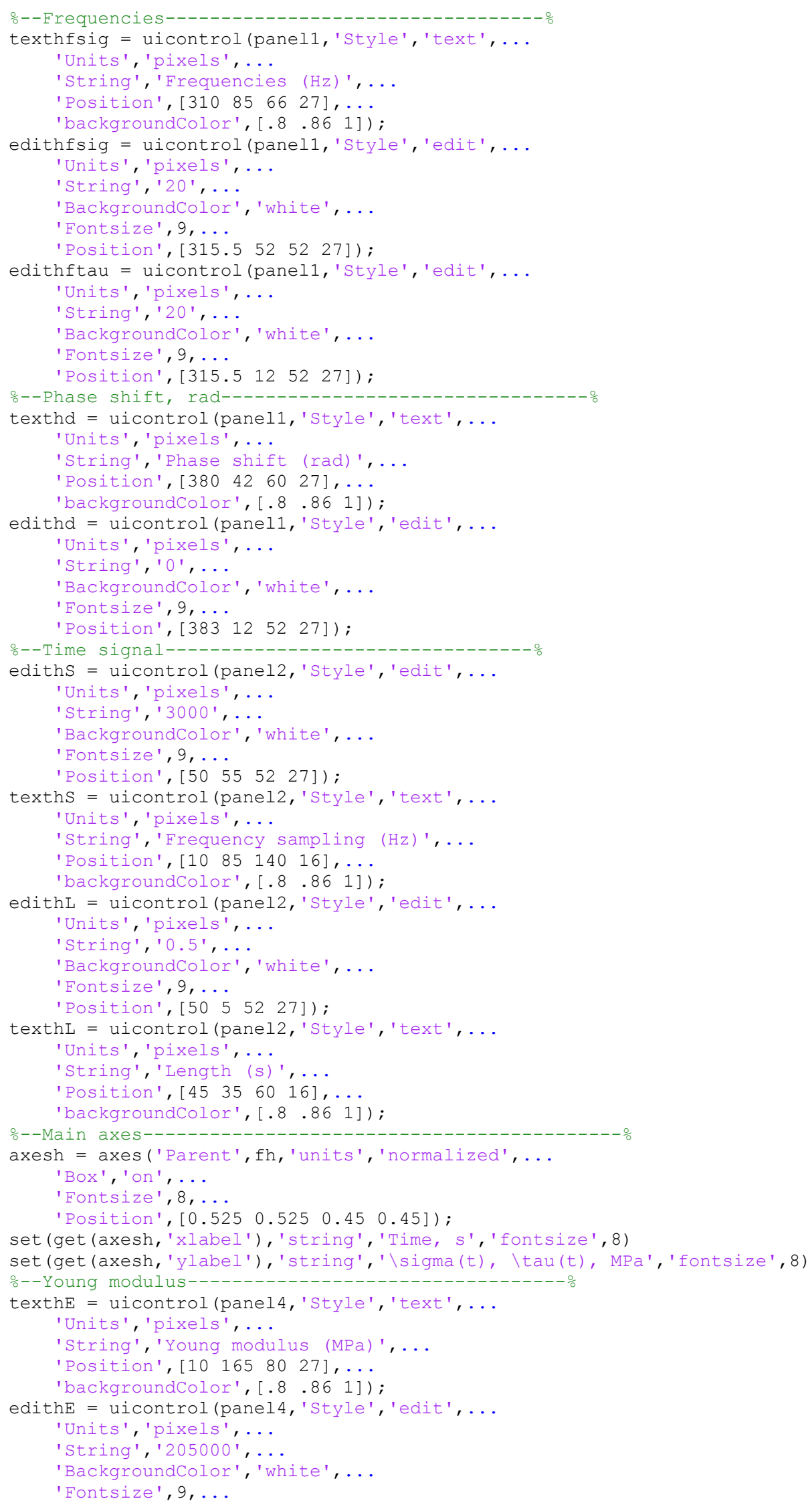




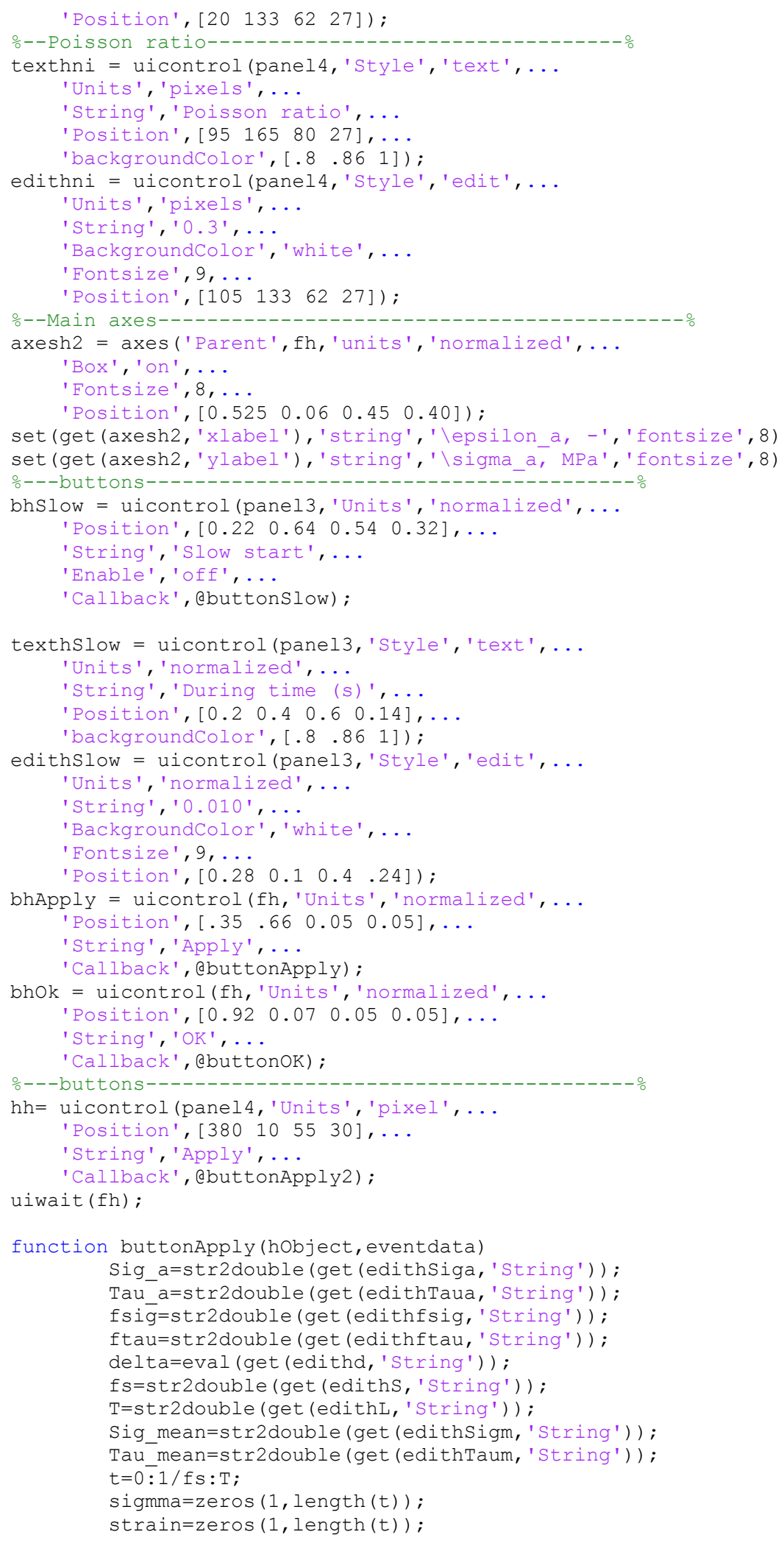




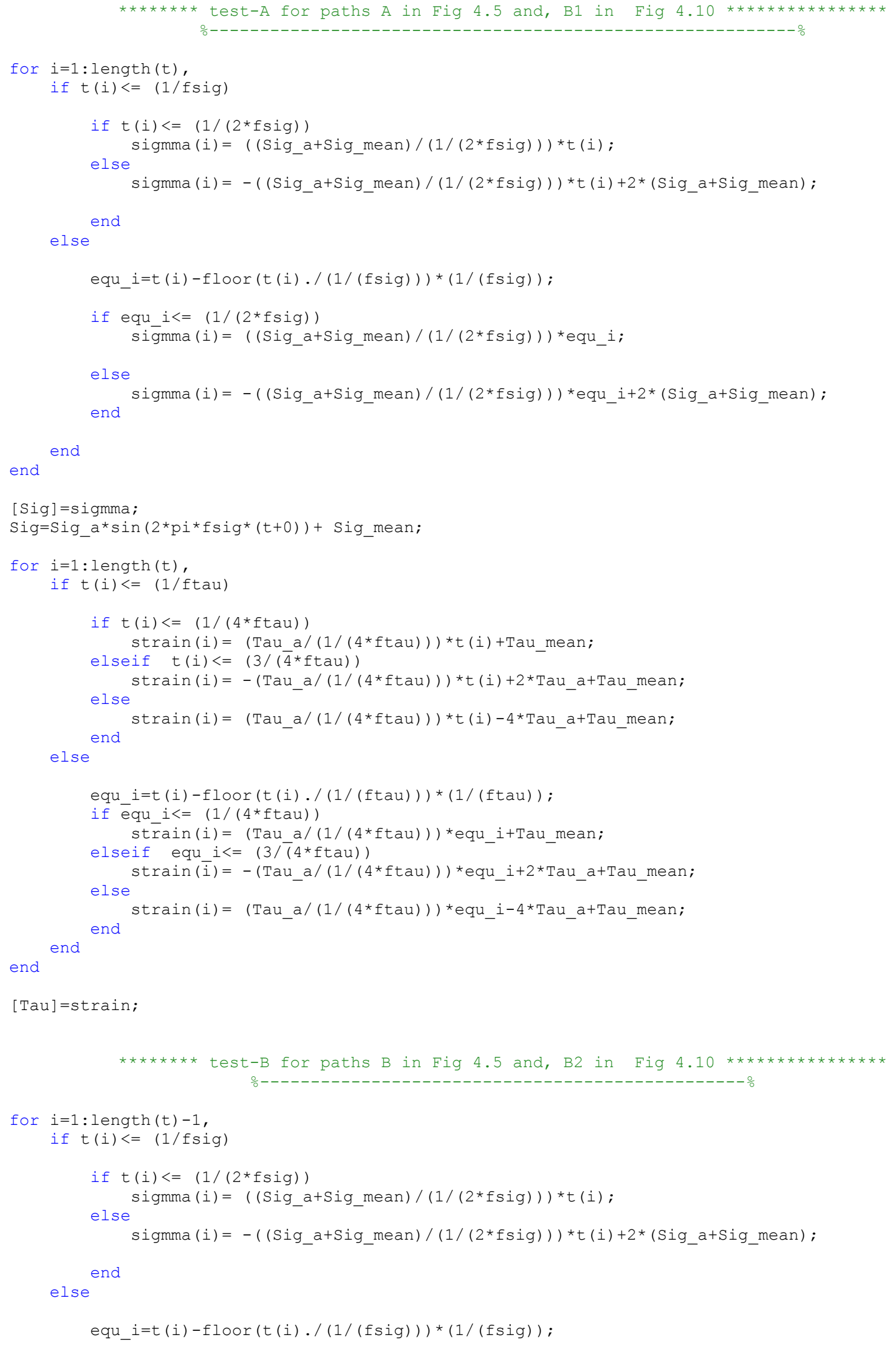




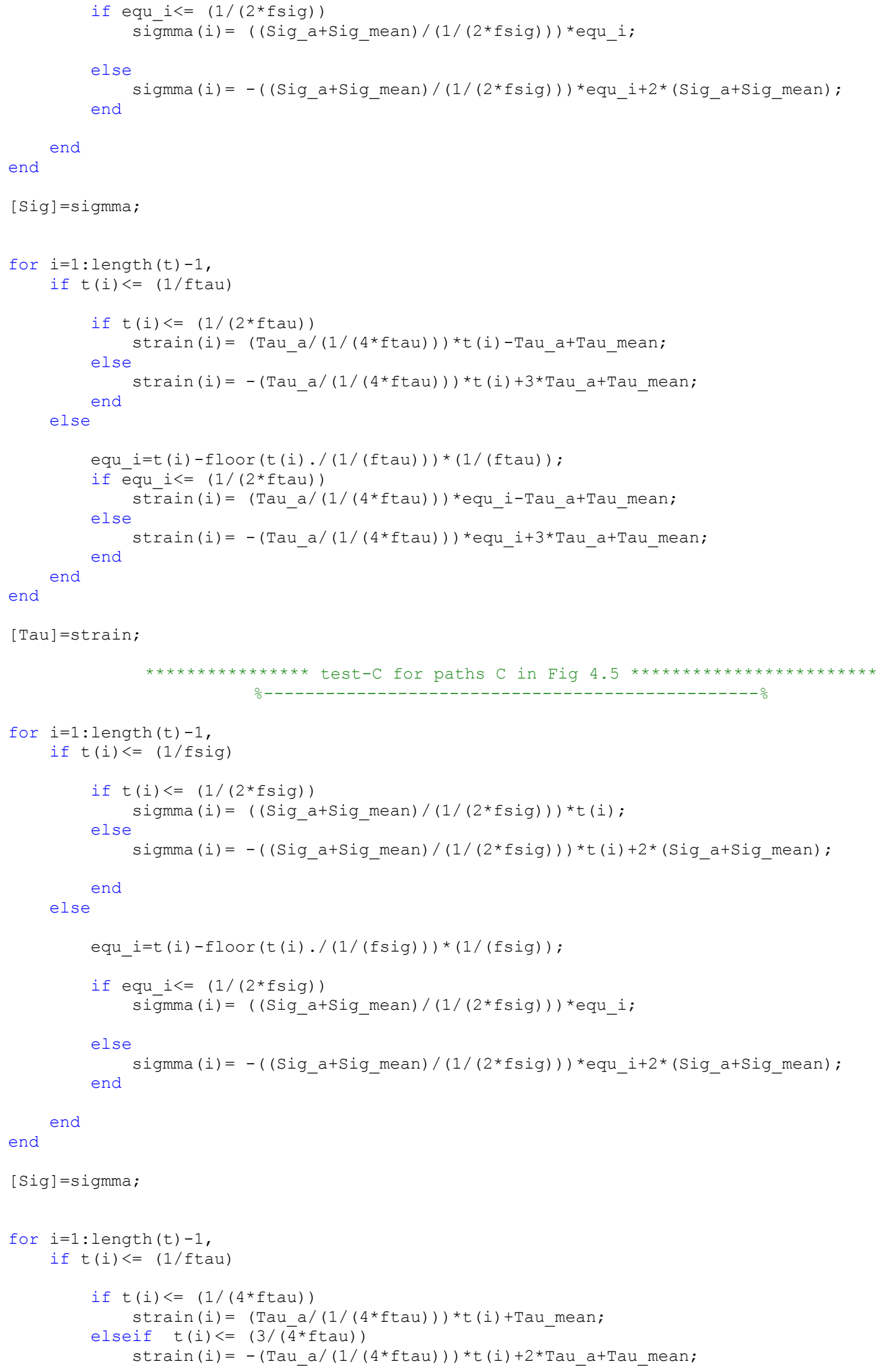




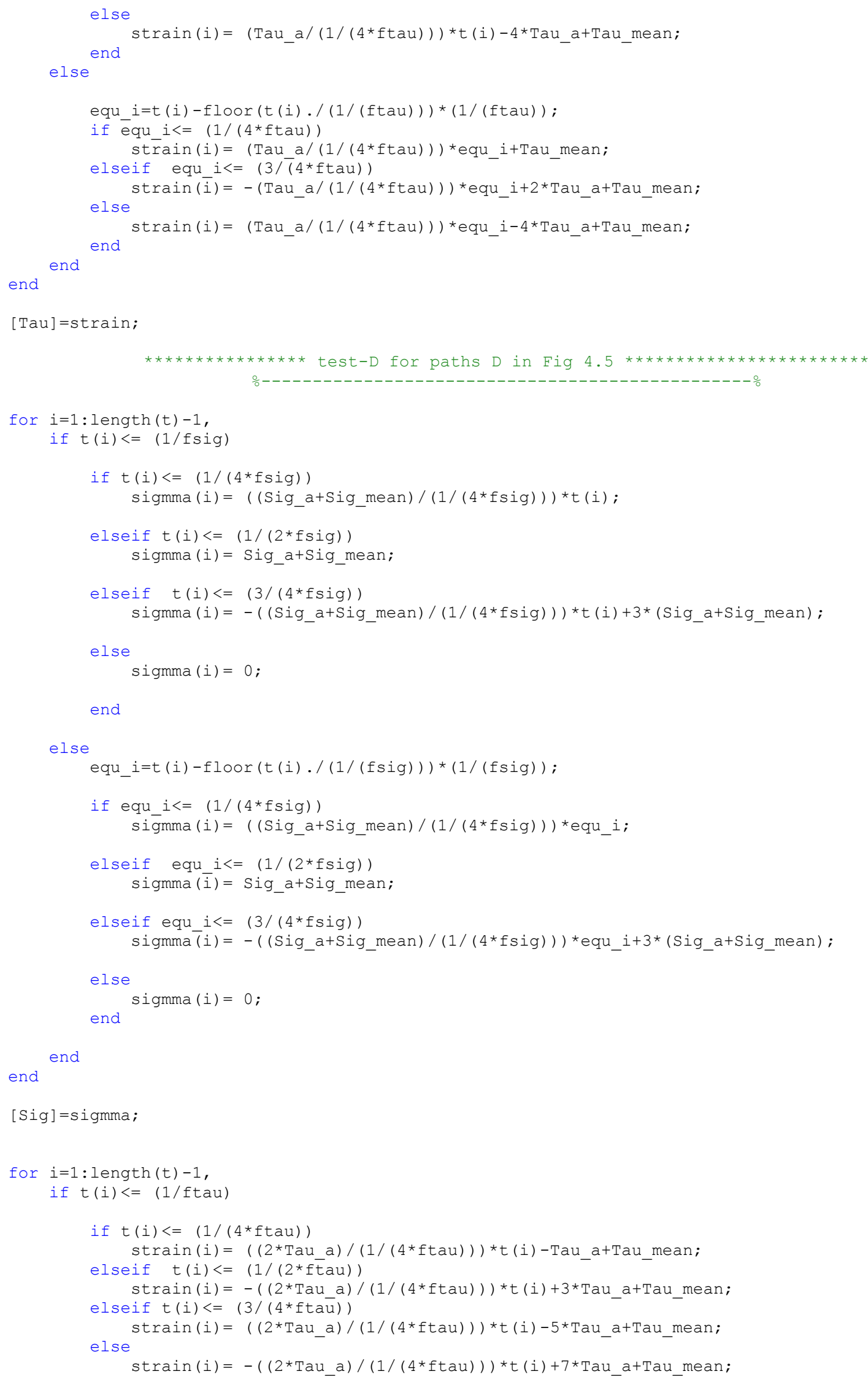




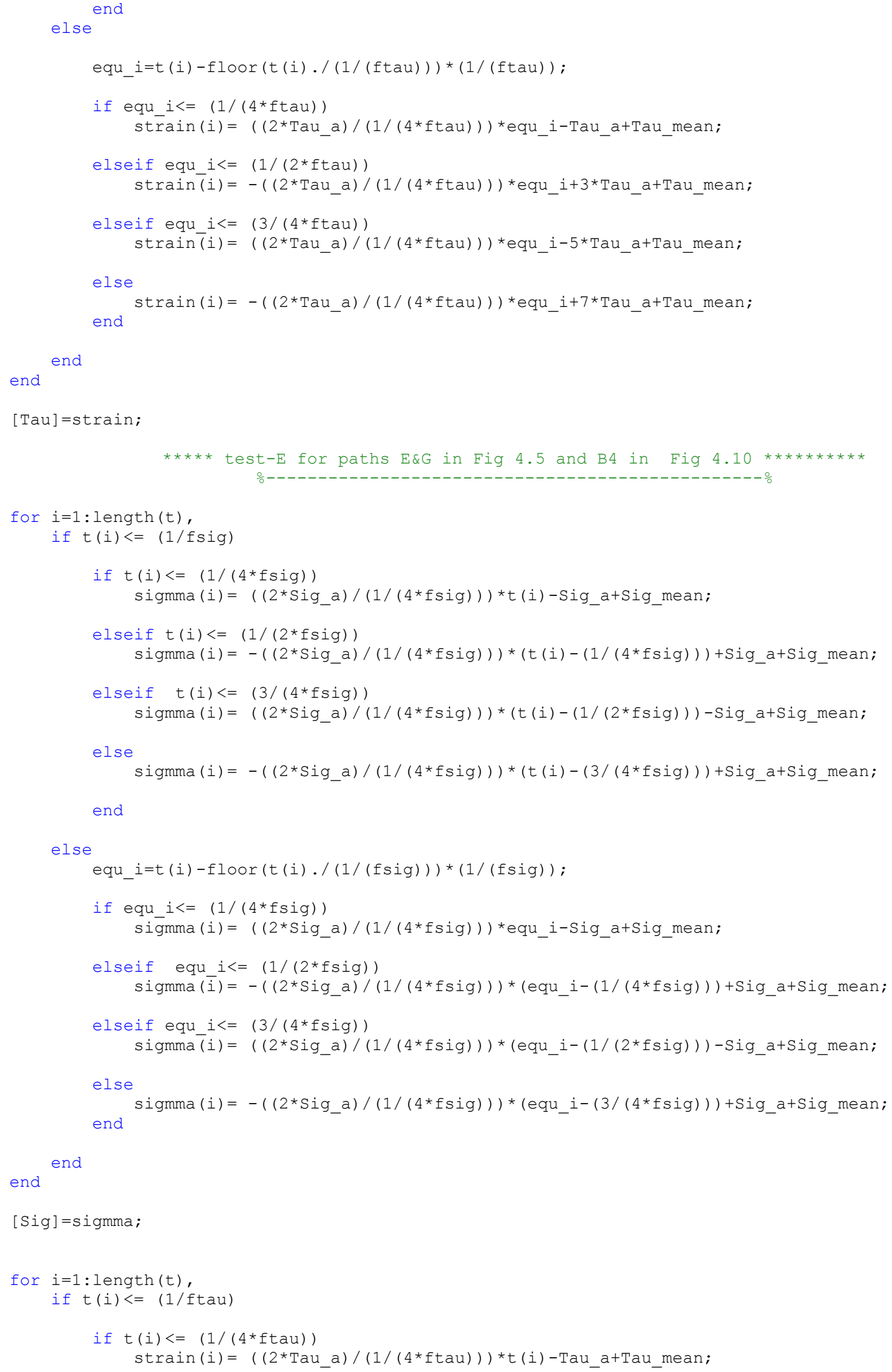




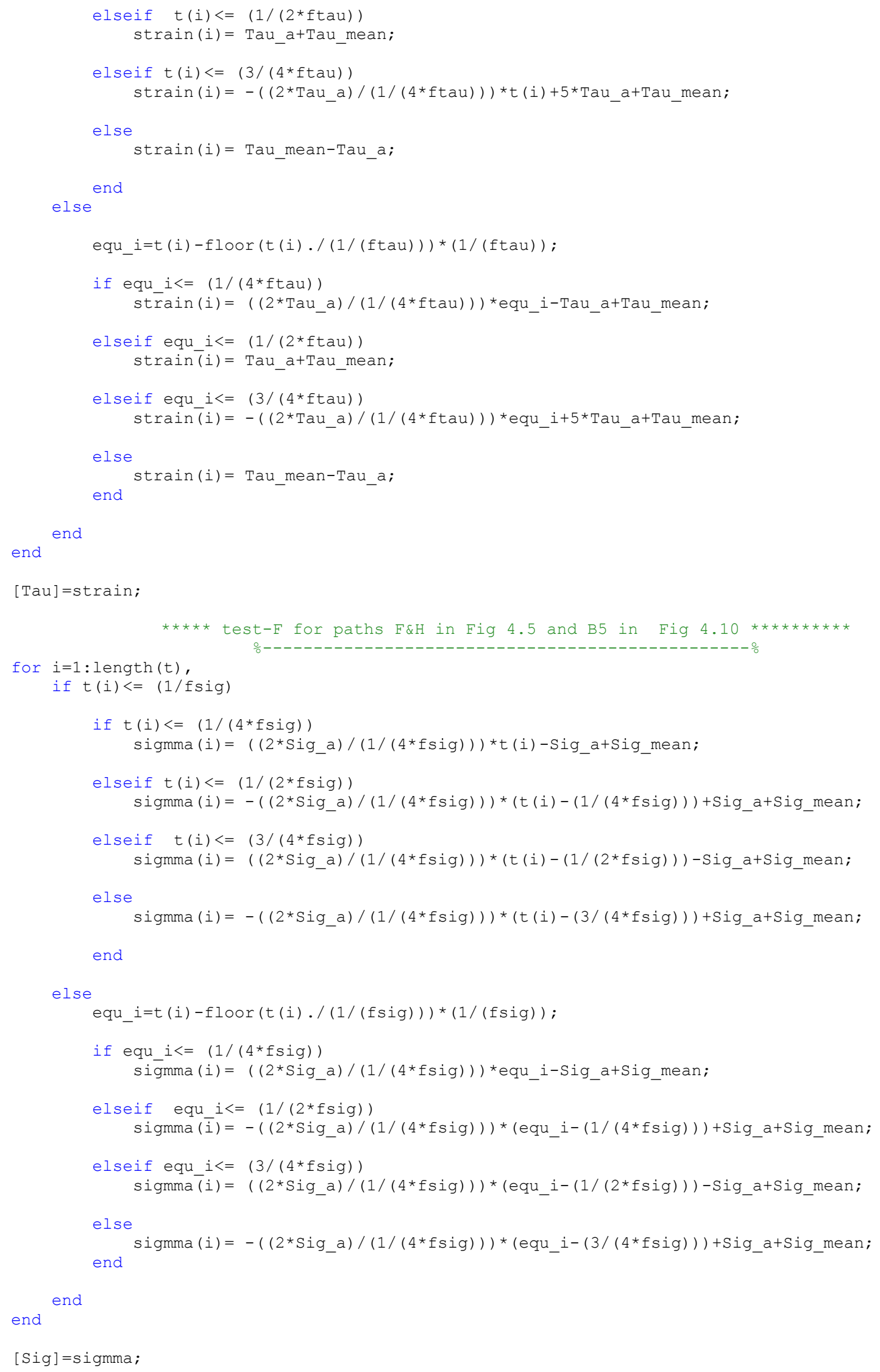




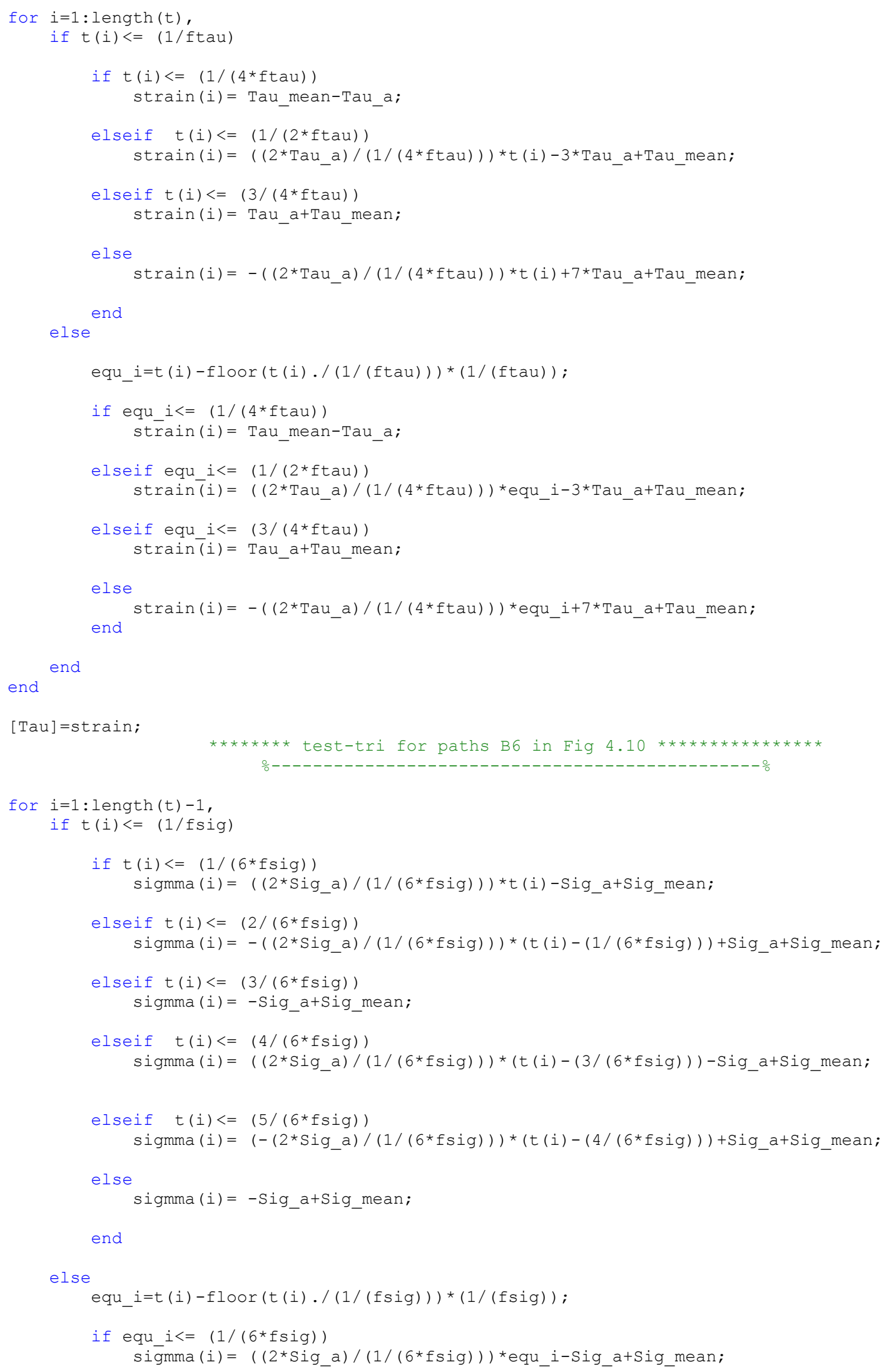




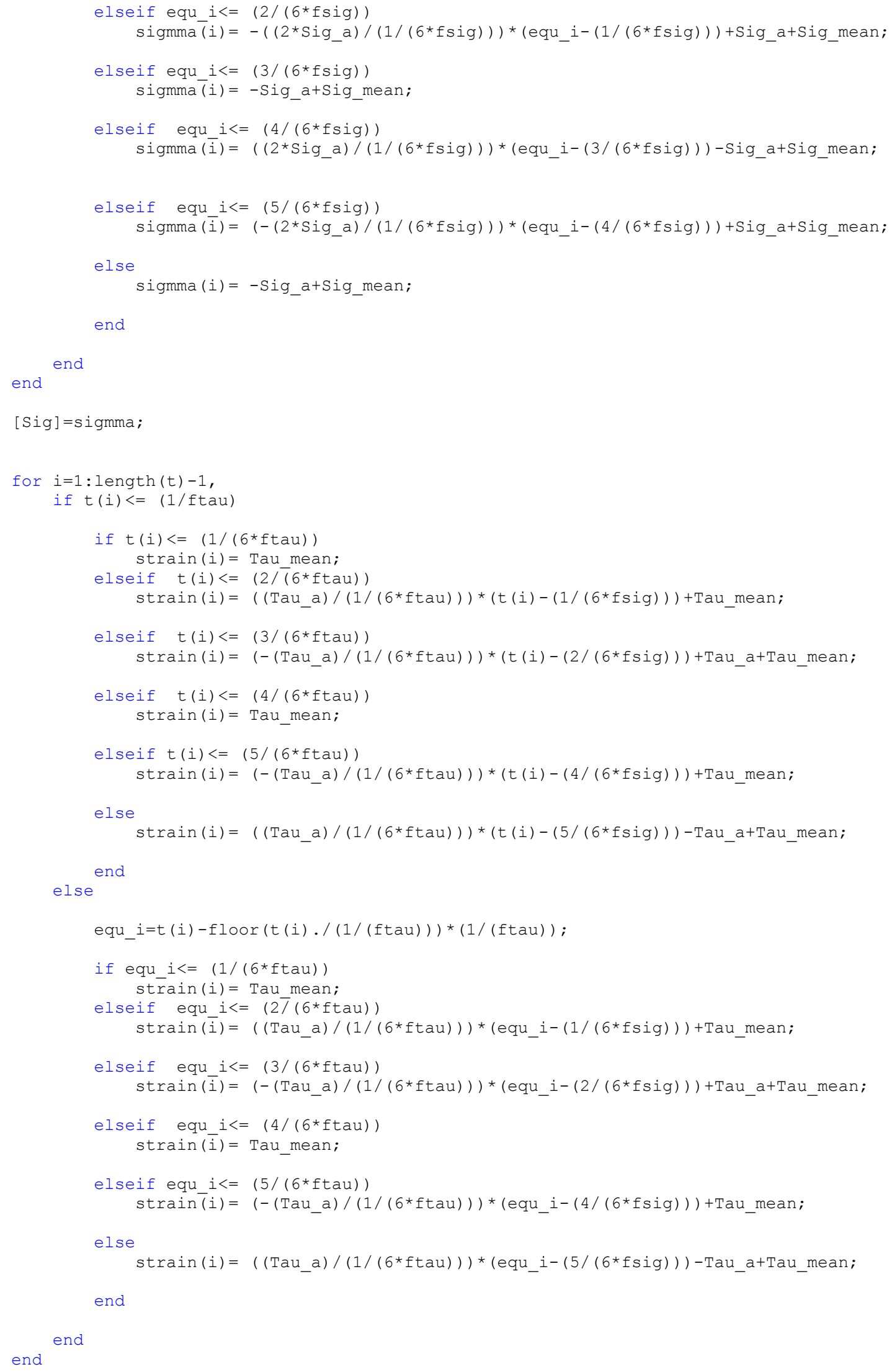




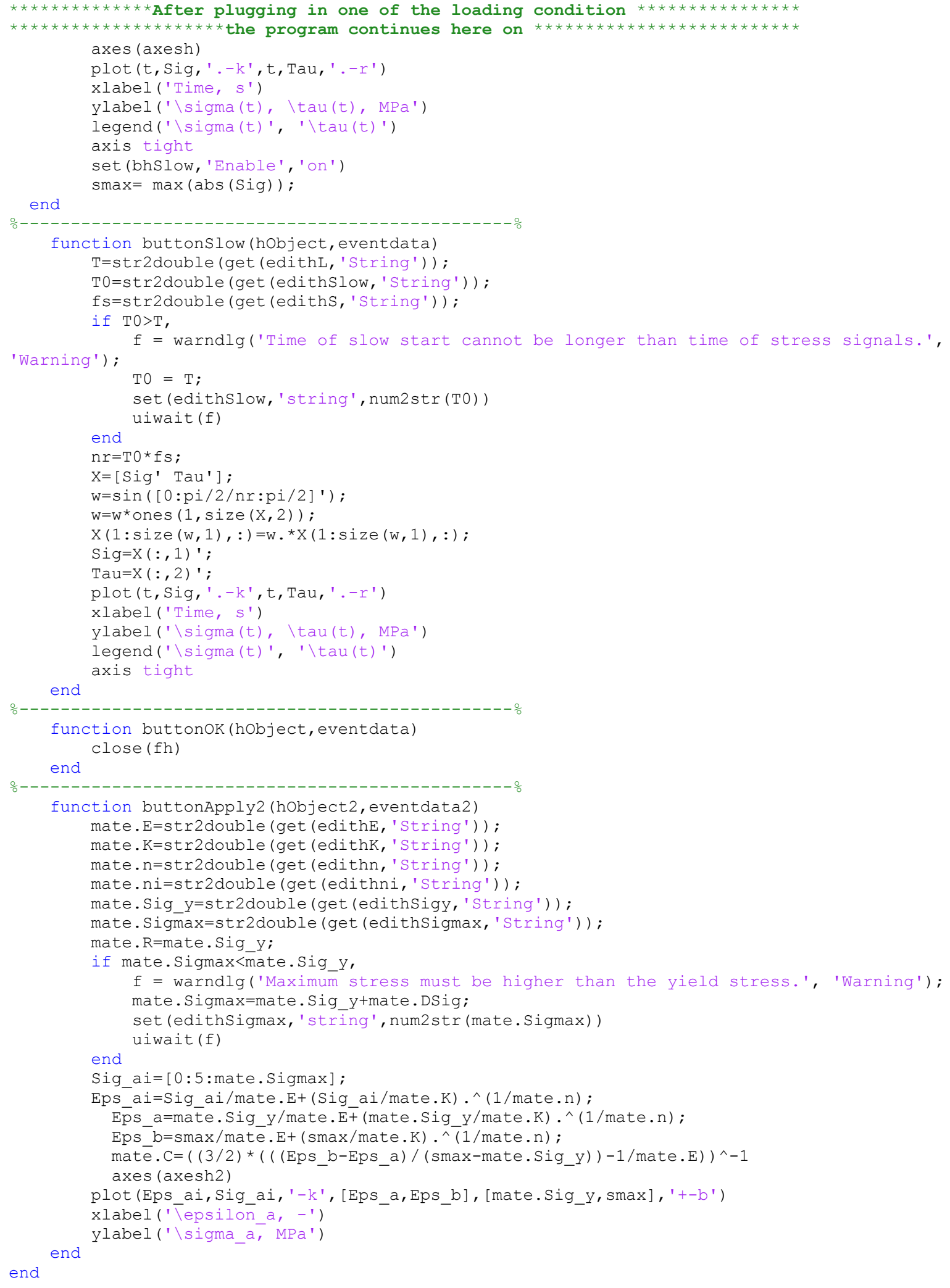




\section{MATLAB Programs for the O-W, McDowell, J-S and C-J-K hardening rules Main program under mixed stress- strain-controlled loading conditions}

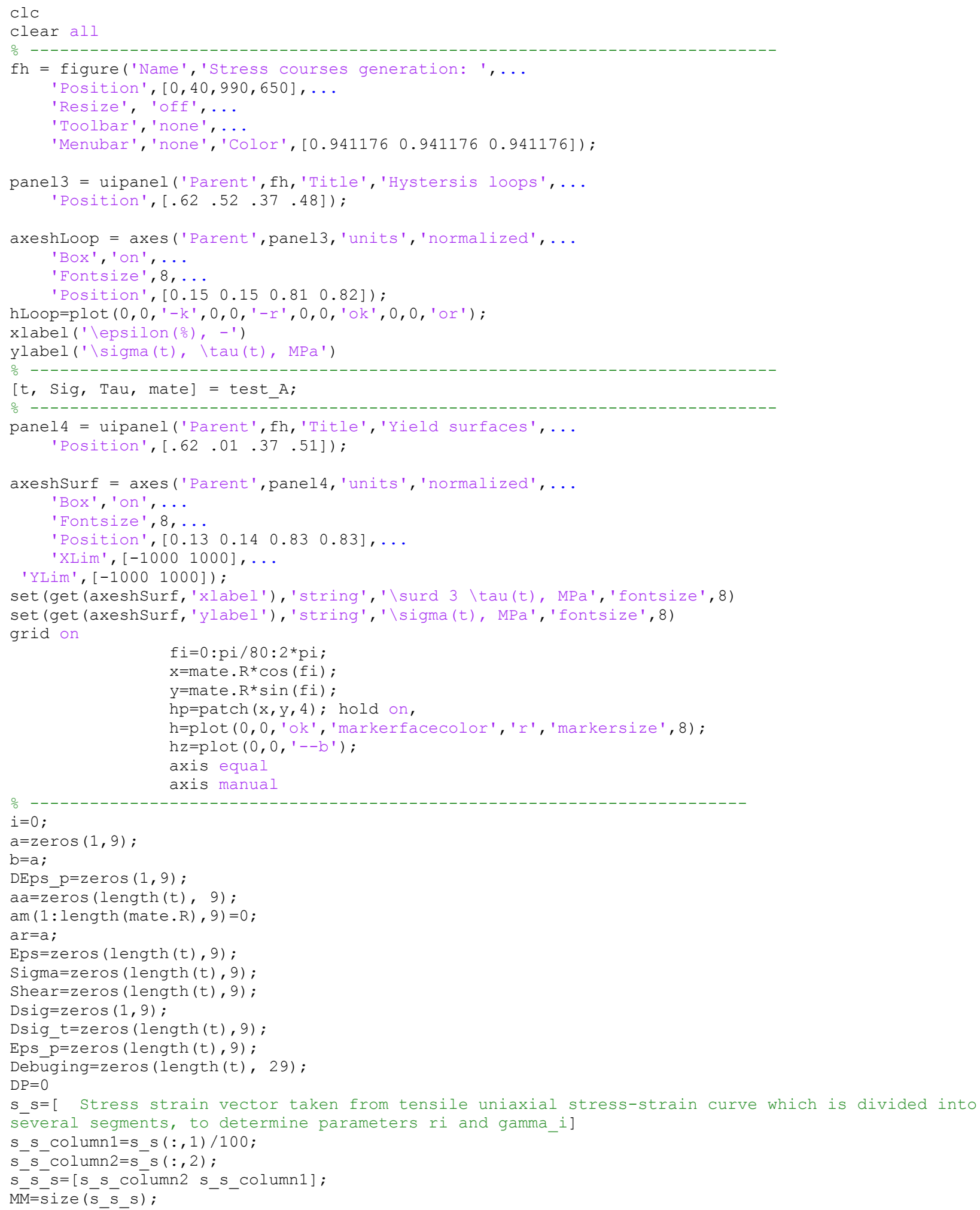




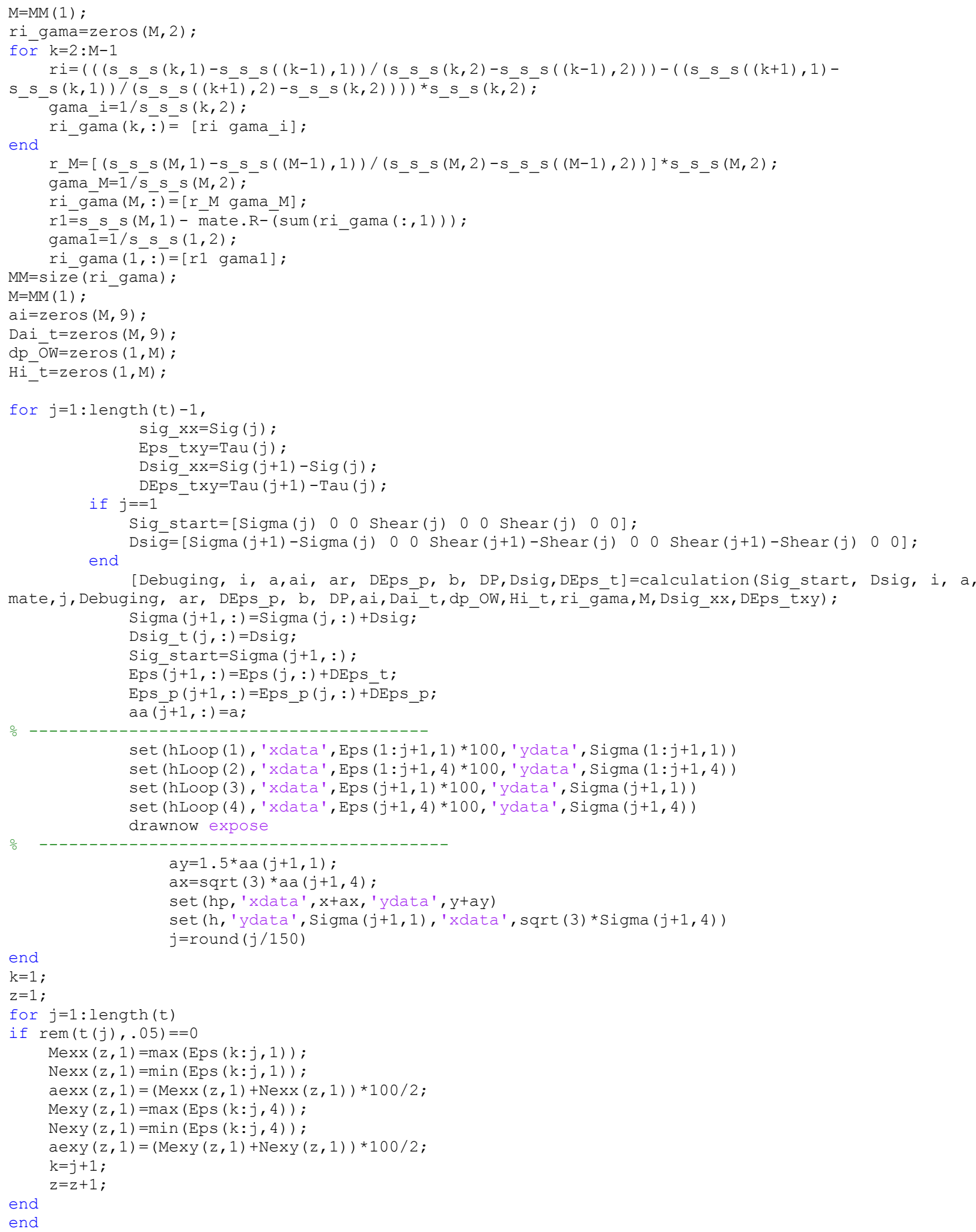




\section{Calculation subroutine for $\mathbf{O}-\mathrm{W}$, McDowell, J-S and C-J-K hardening rules under mixed stress- strain-controlled loading conditions}

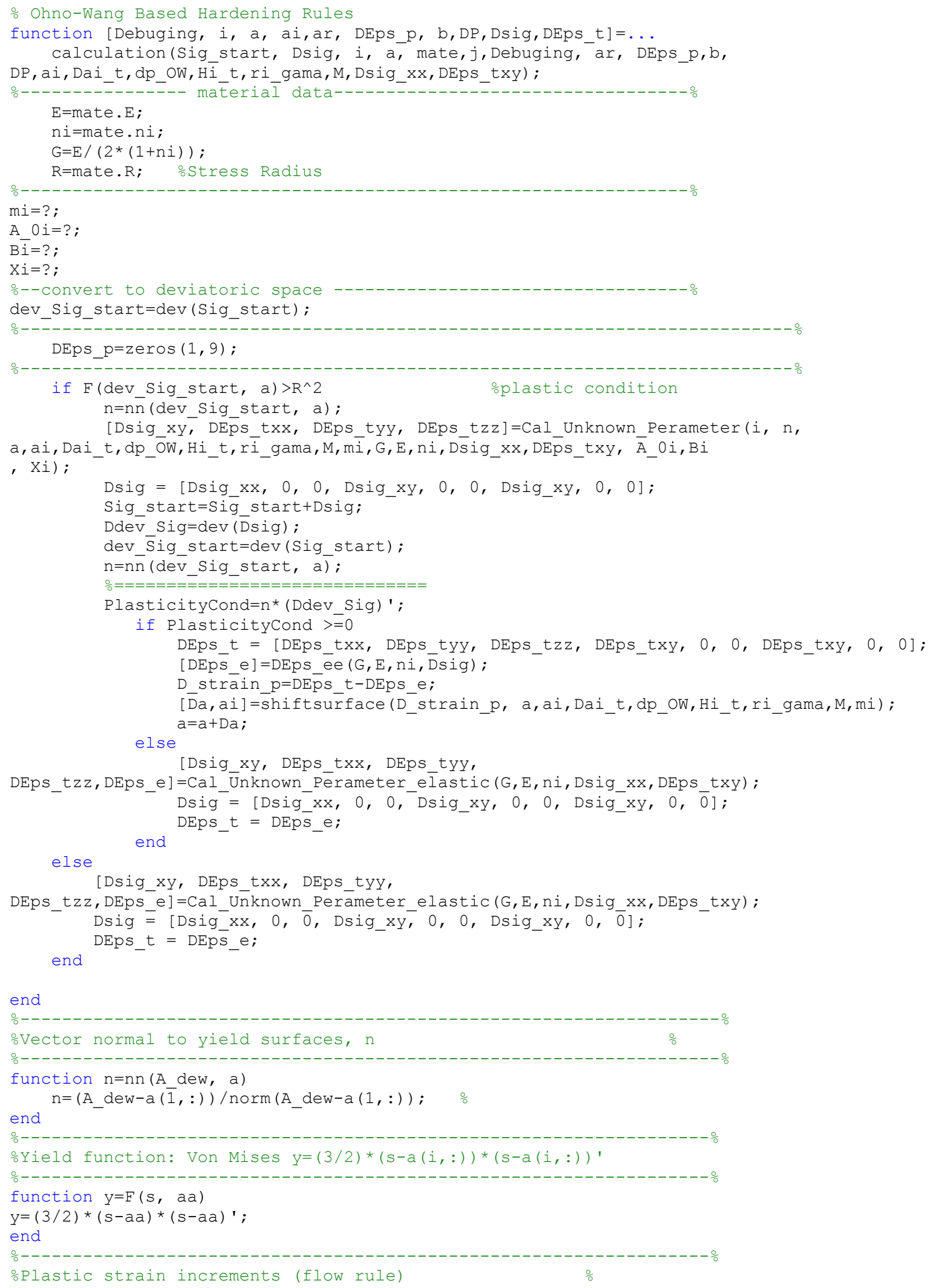




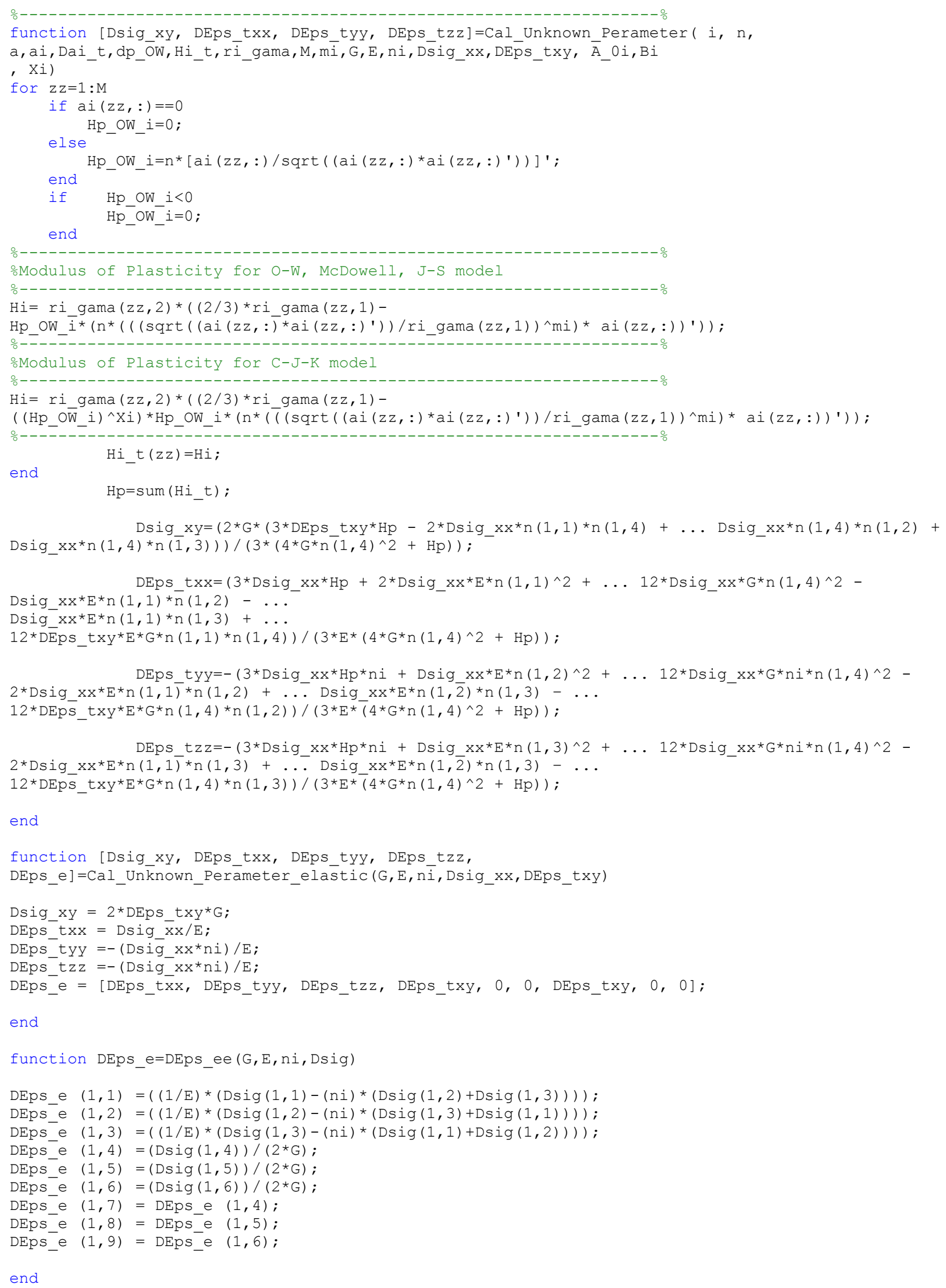




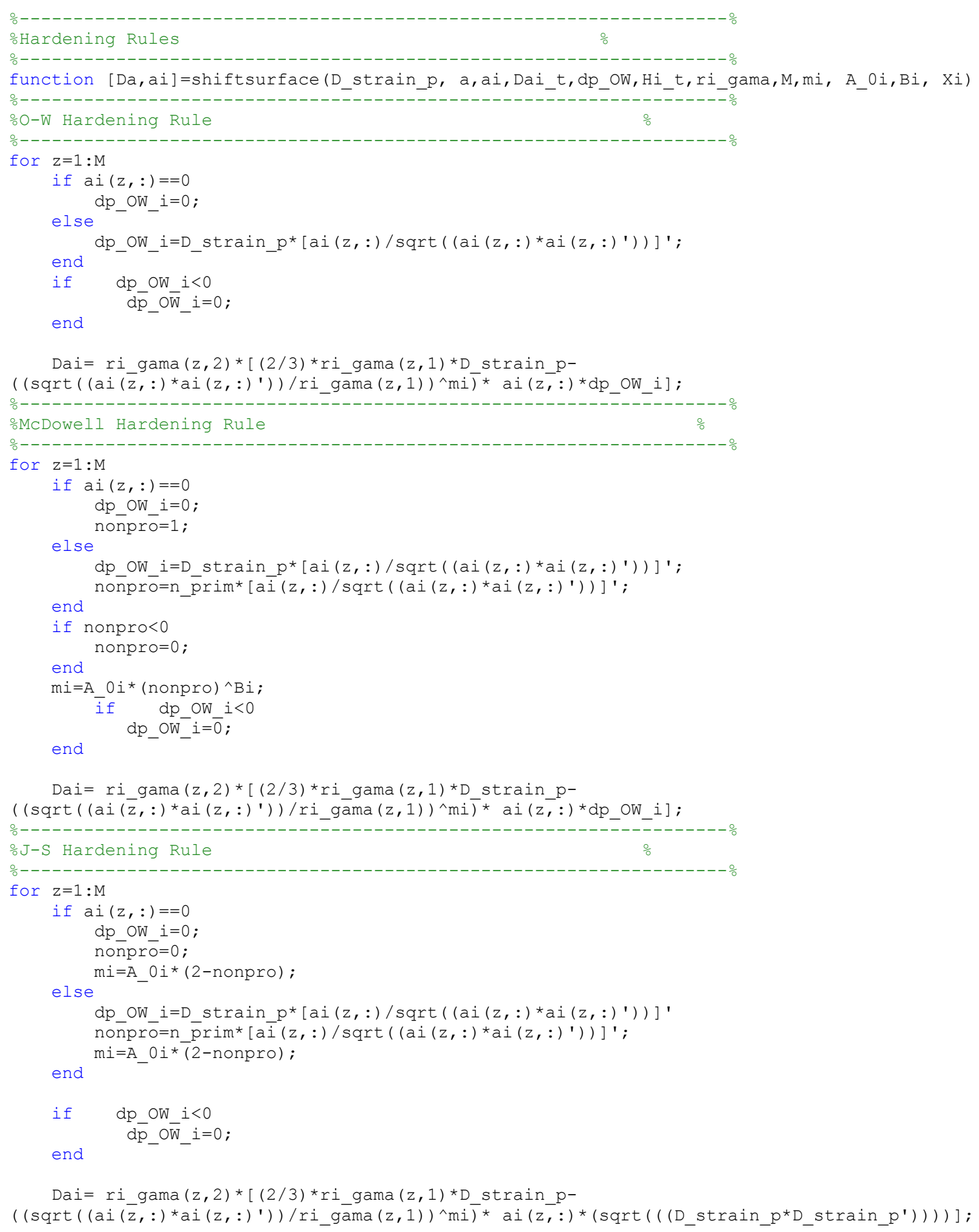




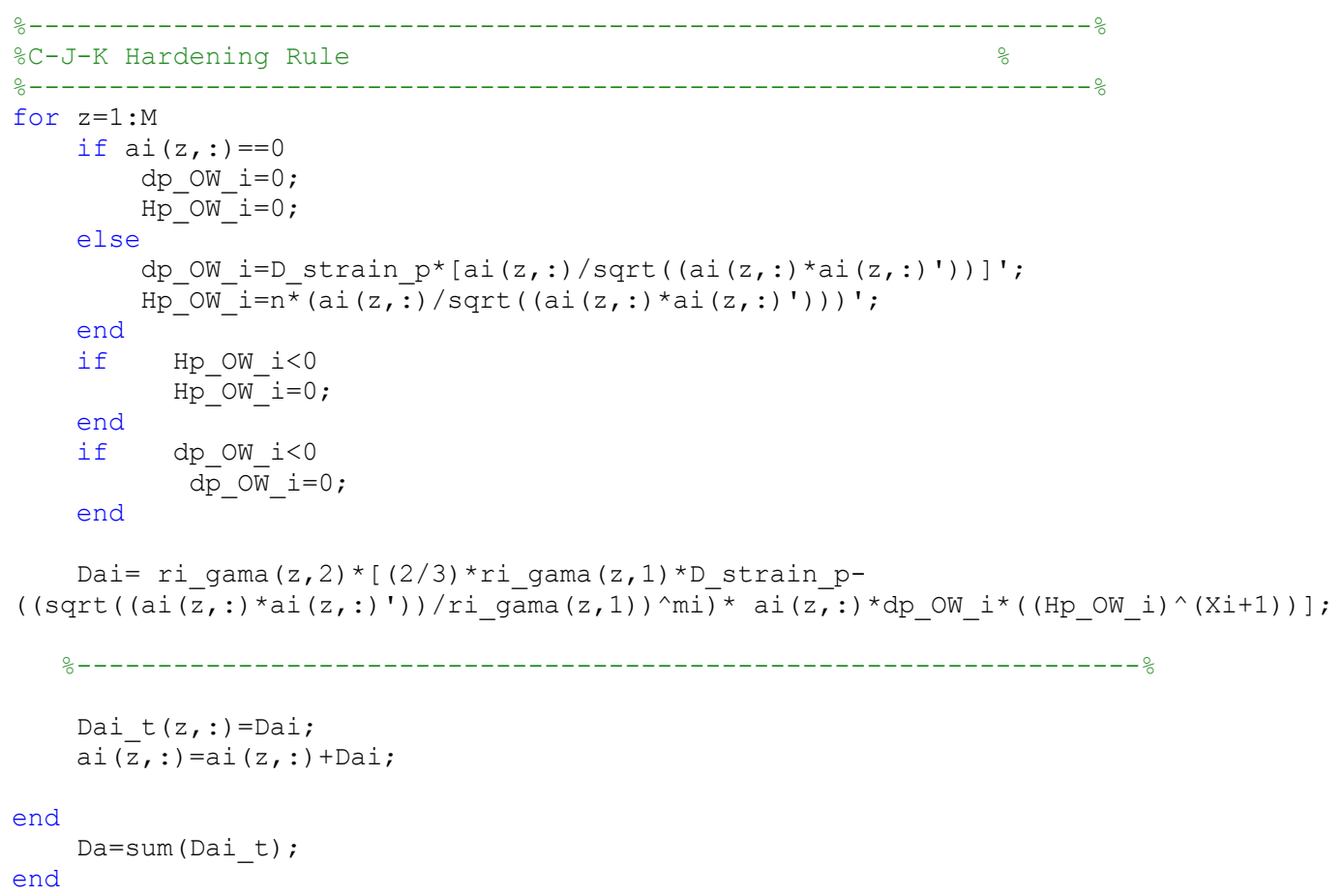




\section{MATLAB Programs for the modified hardening rule}

\section{Main program under stress-controlled loading conditions ${ }^{4}$}

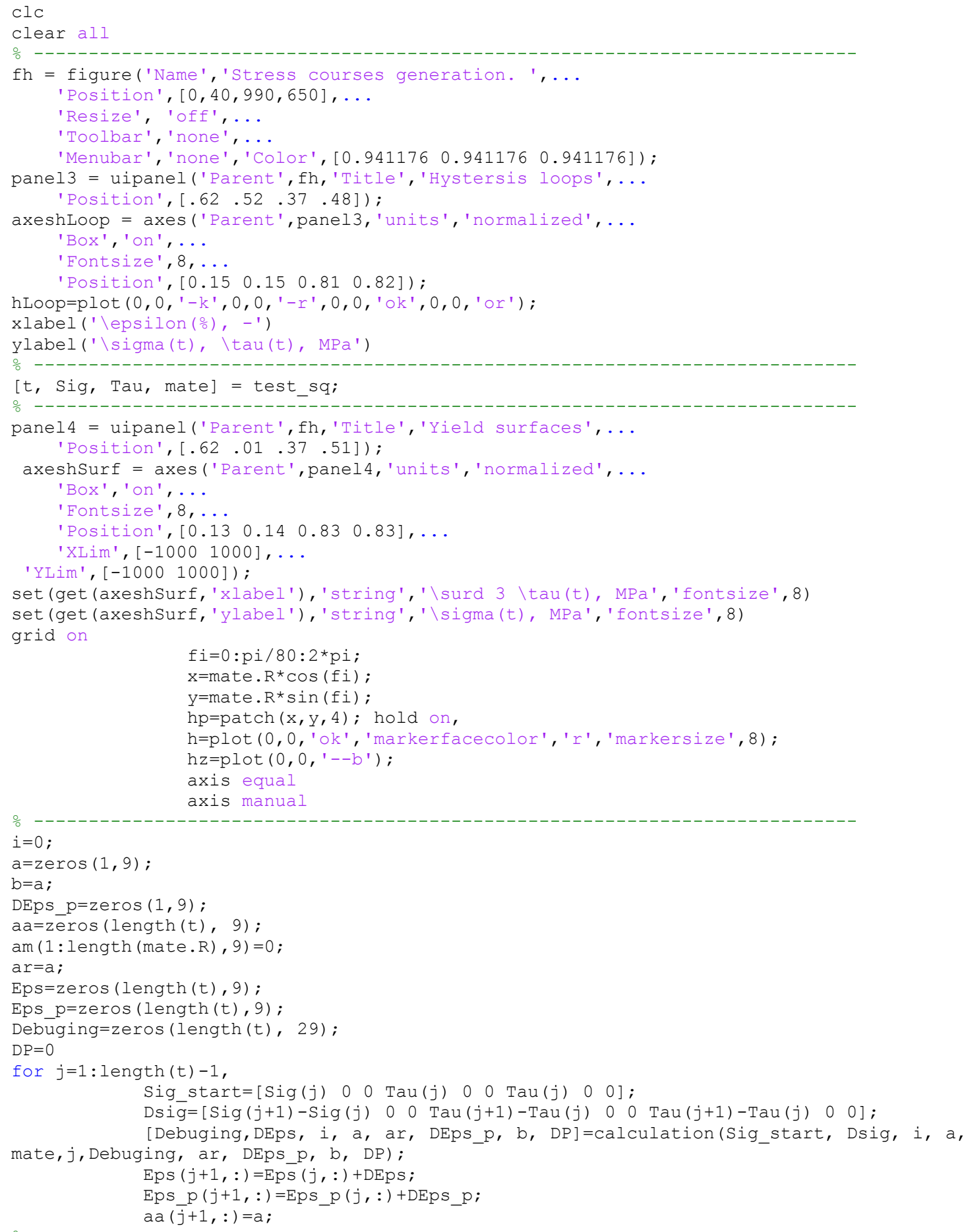

4 This section was taken from [74] 


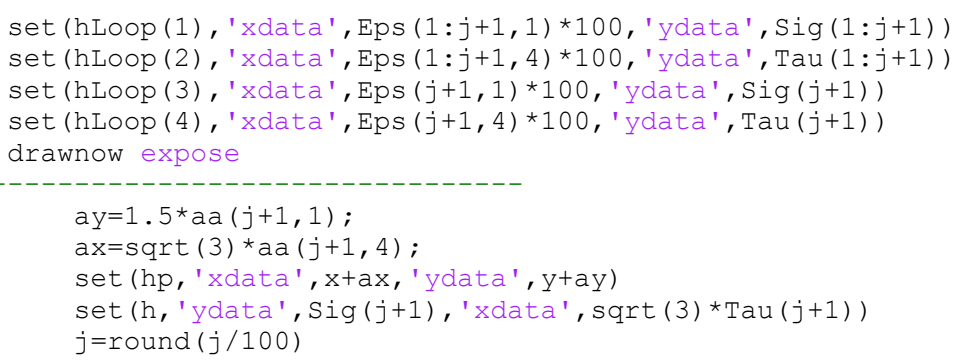

end 


\section{Calculation subroutine for modified model under stress-controlled loading conditions}

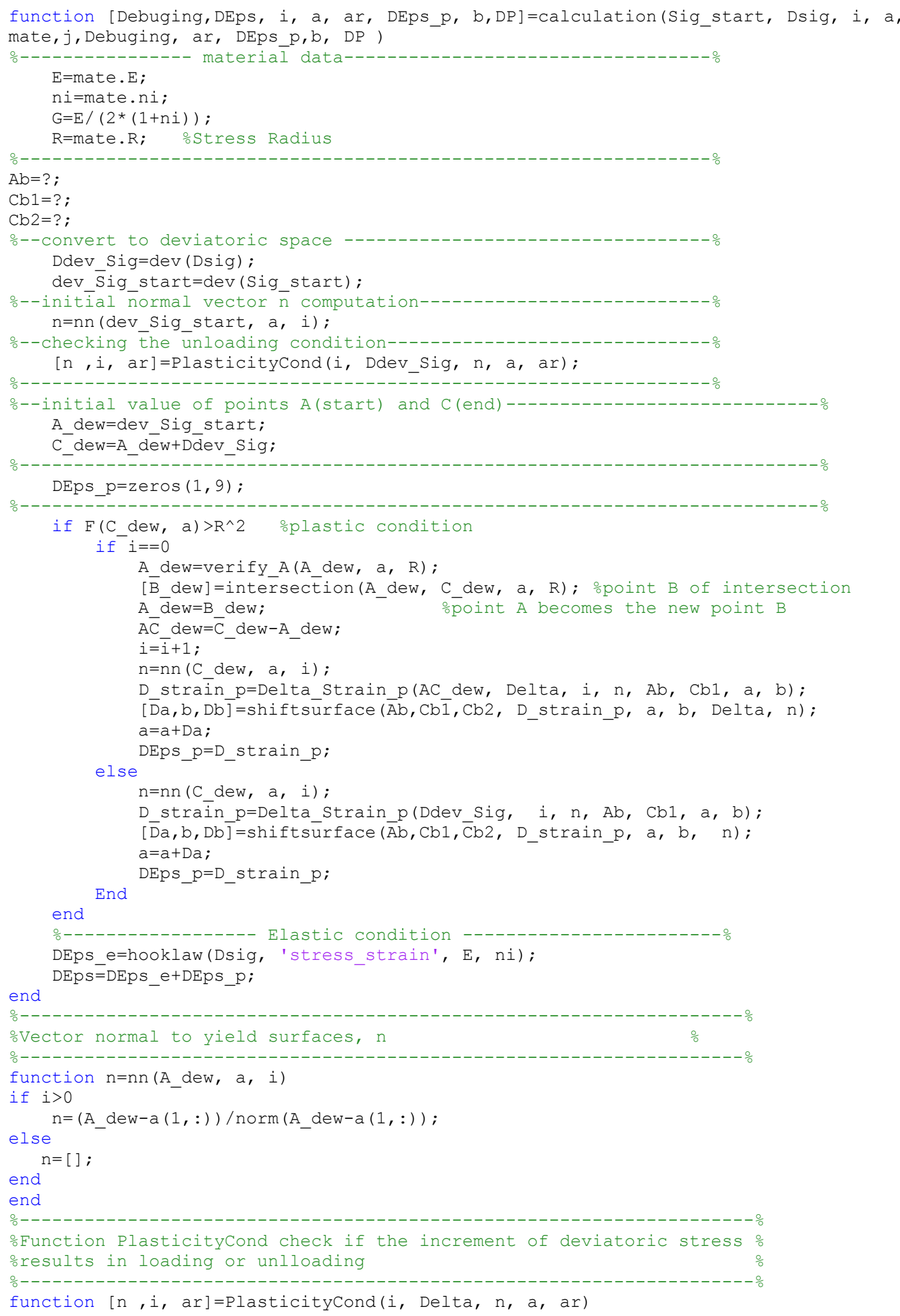




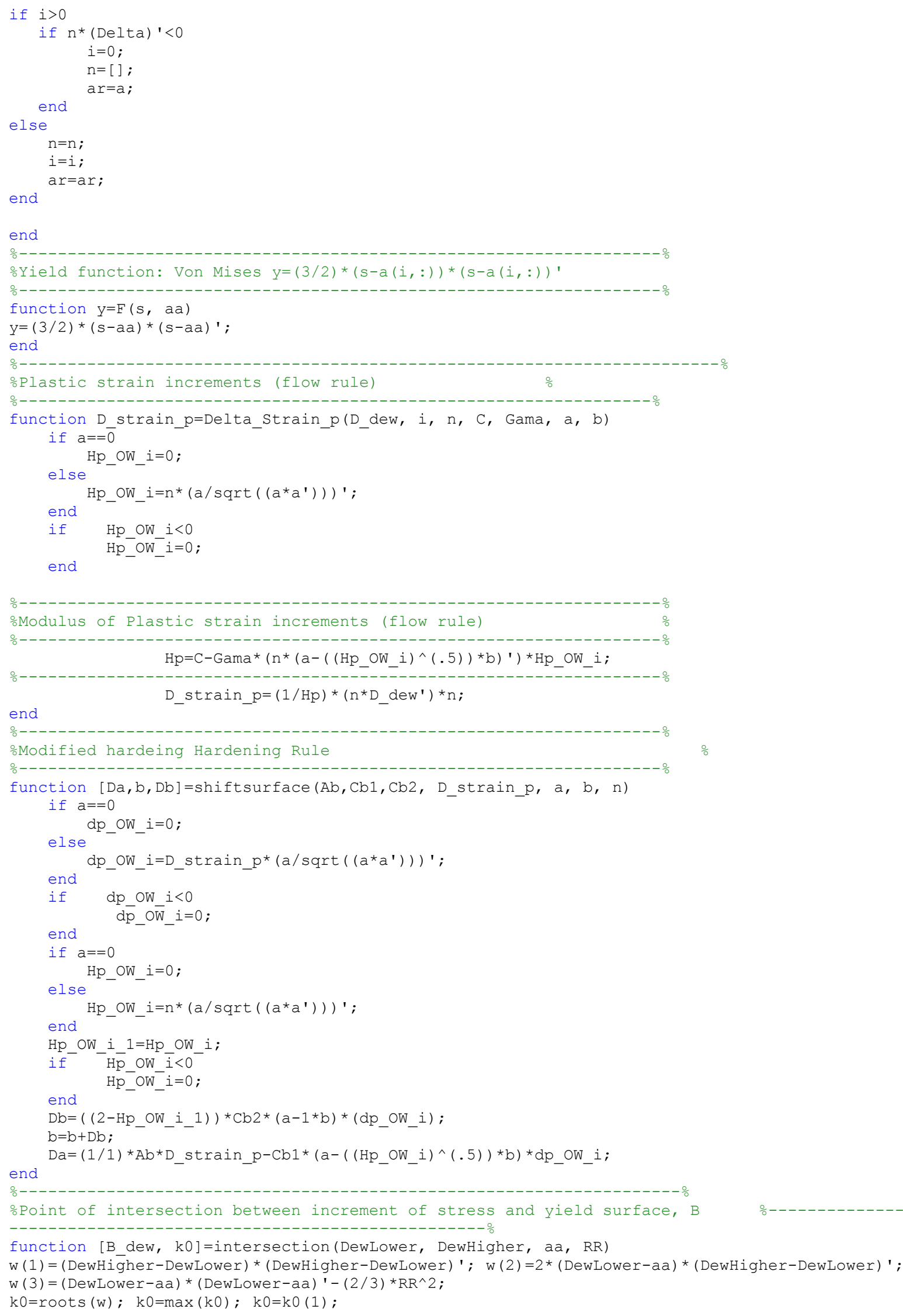




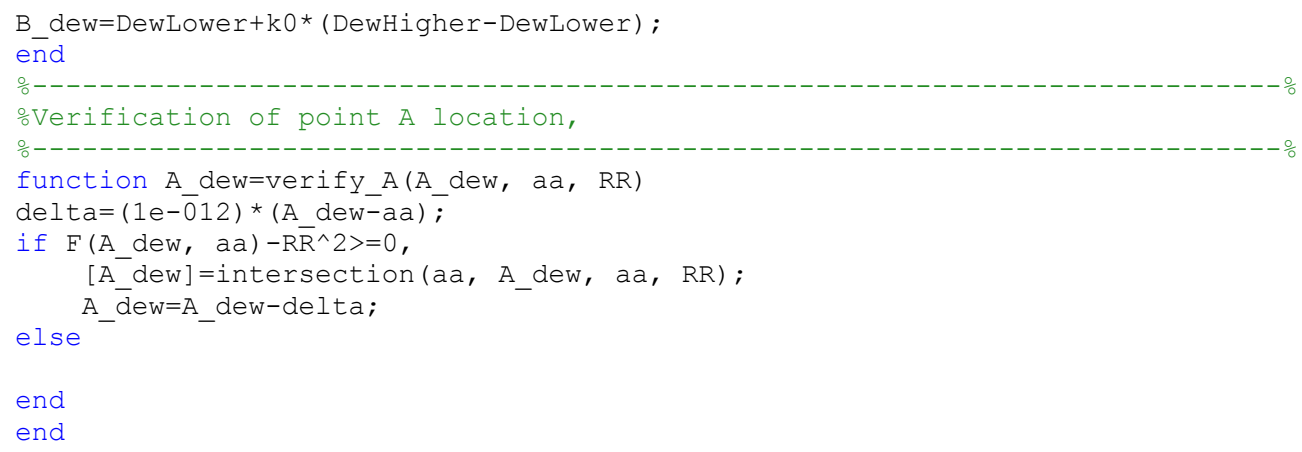




\section{Stress generation subroutine under mixed stress -controlled loading conditions}

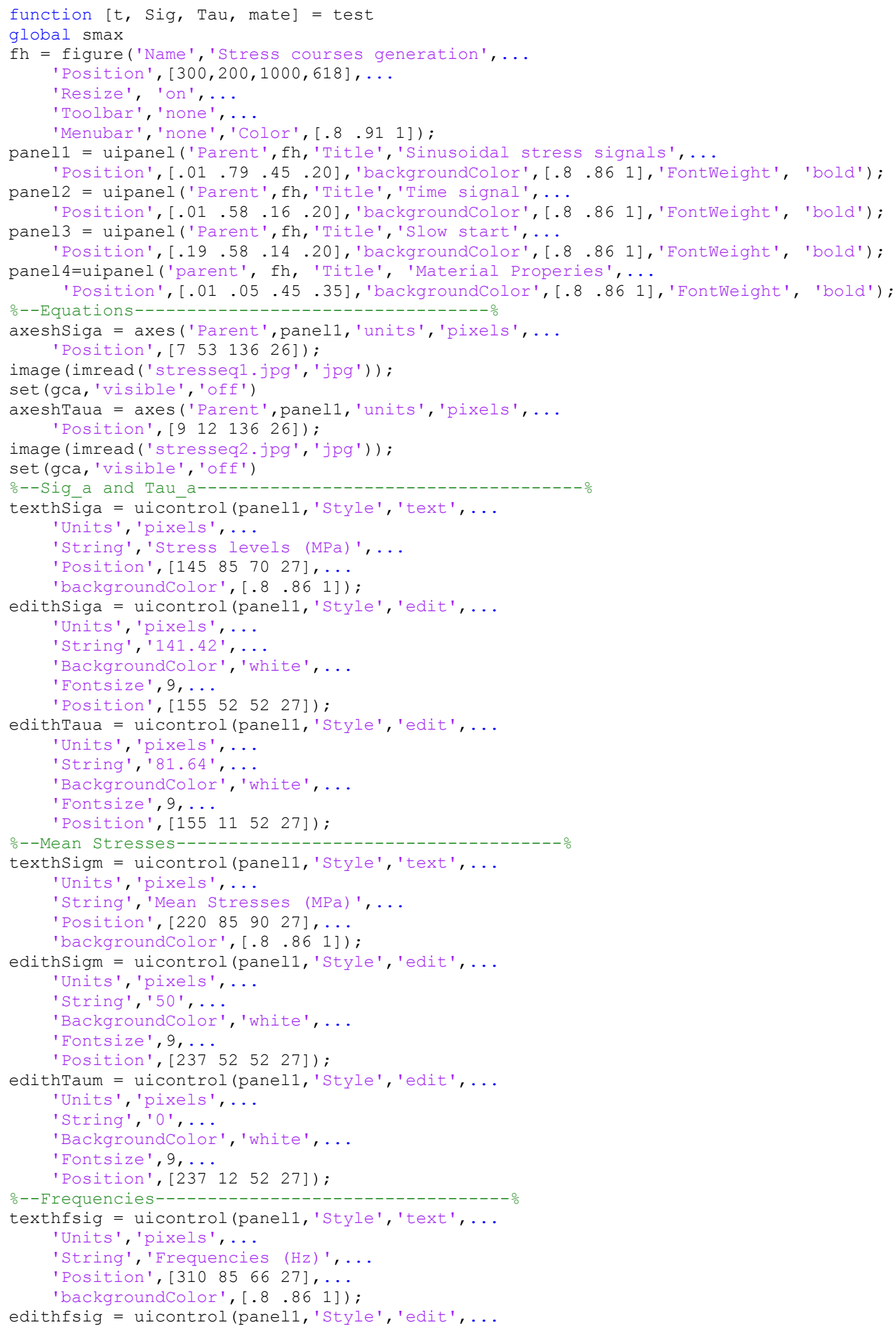




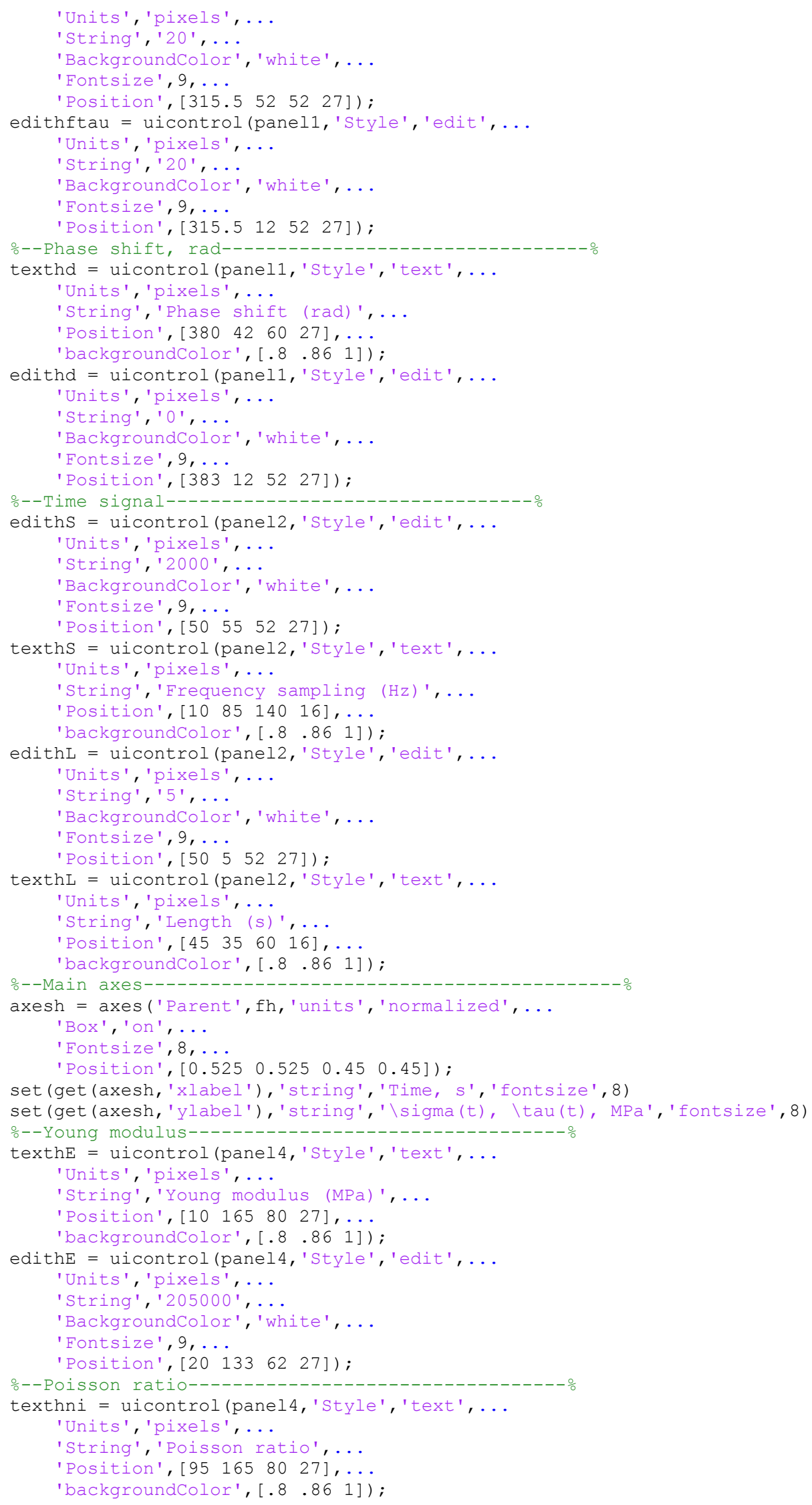




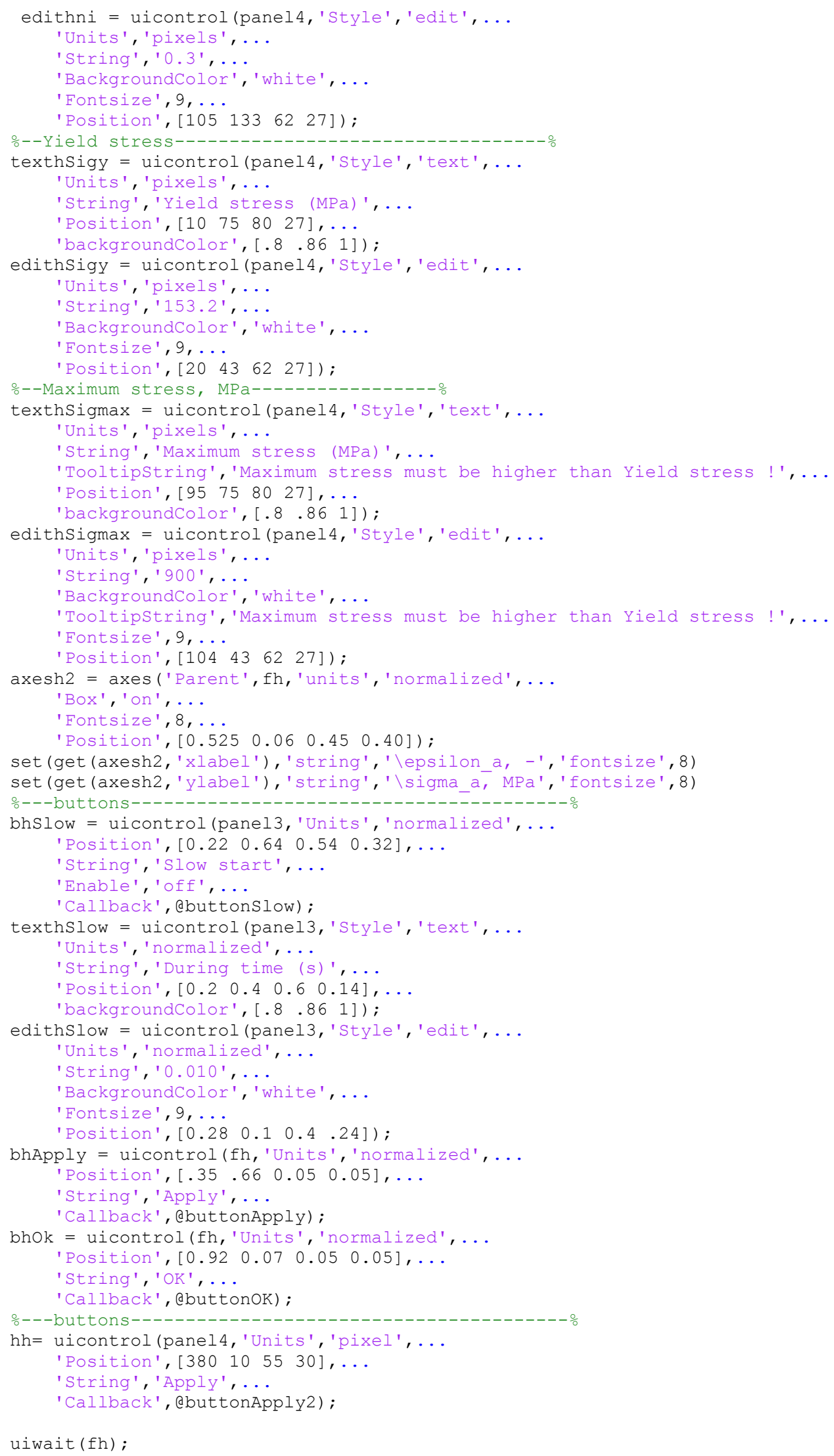


function buttonApply (hobject, eventdata)

Sig_a=str2double (get (edithSiga, 'String'));

Tau a=str2double (get (edithTaua, 'String'));

fsiḡ=str2double (get (edithfsig,'string')) ;

ftau=str2double (get (edithftau, 'String')) ;

delta=eval (get (edithd, 'String'));

fs=str2double (get (ediths, 'String'));

T=str2double (get (edithL, 'String'));

Sig mean=str2double (get (edithSigm, 'string')) ;

Tau_mean=str2double (get (edithTaum, 'String')) ;

$\mathrm{t}=0 \overline{:} 1 / \mathrm{fs}: \mathrm{T}$;

Sig=zeros $(1$, length $(t))$;

Tau=zeros $(1$, length $(t))$;

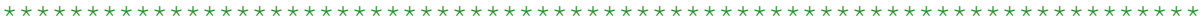

**************** test-sq for paths A2 in Fig $4.9 * * * * * * * * * * * * * * * * * * * * * * * *$

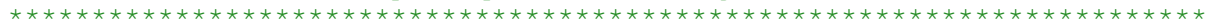

for $i=1:$ length $(t)$

if $t(i)<=(1 /$ fsig)

if $t(i)<=(1 /(4 *$ fsig $))$

Sig $(i)=$ Sig_a+Sig_mean;

elseif $t(i)<=(1 /(2 * f \operatorname{sig}))$

Sig $(i)=-((2 *$ Sig_a $) /(1 /(4 * f \operatorname{sig}))) *(t(i)-(1 /(4 * f \operatorname{sig})))+$ Sig_a+Sig_mean;

elseif $t(i)<=(3 /(4 *$ fsig) $)$

Sig $(i)=-$ Sig_a+Sig_mean;

else

$\operatorname{Sig}(i)=((2 * \operatorname{Sig} a) /(1 /(4 * f \operatorname{Sig}))) *(t(i)-(3 /(4 *$ Sig $)))-$ Sig_a+Sig_mean

end

else

equ_i=t(i)-floor(t(i)./(1/(fsig)))*(1/(fsig));

if equ $i<=(1 /(4 *$ fsig) $)$

$\operatorname{Sig}(i)=$ Sig_a+Sig_mean;

elseif equ_i<= $(1 /(2 *$ fsig $))$

$\operatorname{Sig}(i)=-((2 * \operatorname{Sig}$ _a $) /(1 /(4 *$ fsig $))) *($ equ_i- $(1 /(4 *$ fsig $)))+$ Sig_a+Sig_mean;

elseif equ $i<=(3 /(4 *$ fsig) $)$

Sig $(i)=-$ Sig_a+Sig_mean;

else

end

$\operatorname{Sig}(i)=((2 *$ Sig_a $) /(1 /(4 * f \operatorname{sig}))) *($ equ_i- $(3 /(4 *$ fsig $)))-S i g \_a+S i g \_m e a n$;

end

end

for $i=1$ : length $(t)$,

if $t(i)<=(1 /$ ftau $)$

if $\mathrm{t}(\mathrm{i})<=(1 /(4 * \mathrm{ftau}))$

$\operatorname{Tau}(i)=\left(\left(2 \star T a u \_a\right) /(1 /(4 * f t a u))\right) * t(i)-T a u \_a+T a u \_m e a n ;$

elseif $t(i)<=(1 /(2 * f t a u))$

Tau $(i)=$ Tau_a+Tau_mean;

elseif $t(i)<=(3 /(4 *$ ftau $)$

$\operatorname{Tau}(i)=-((2 *$ Tau_a $) /(1 /(4 * \mathrm{ftau}))) *($ t $(i)-(1 /(2 * \mathrm{ftau})))+$ Tau_a+Tau_mean;

else

Tau $(i)=-T a u \_a+T a u \_m e a n ;$

end 


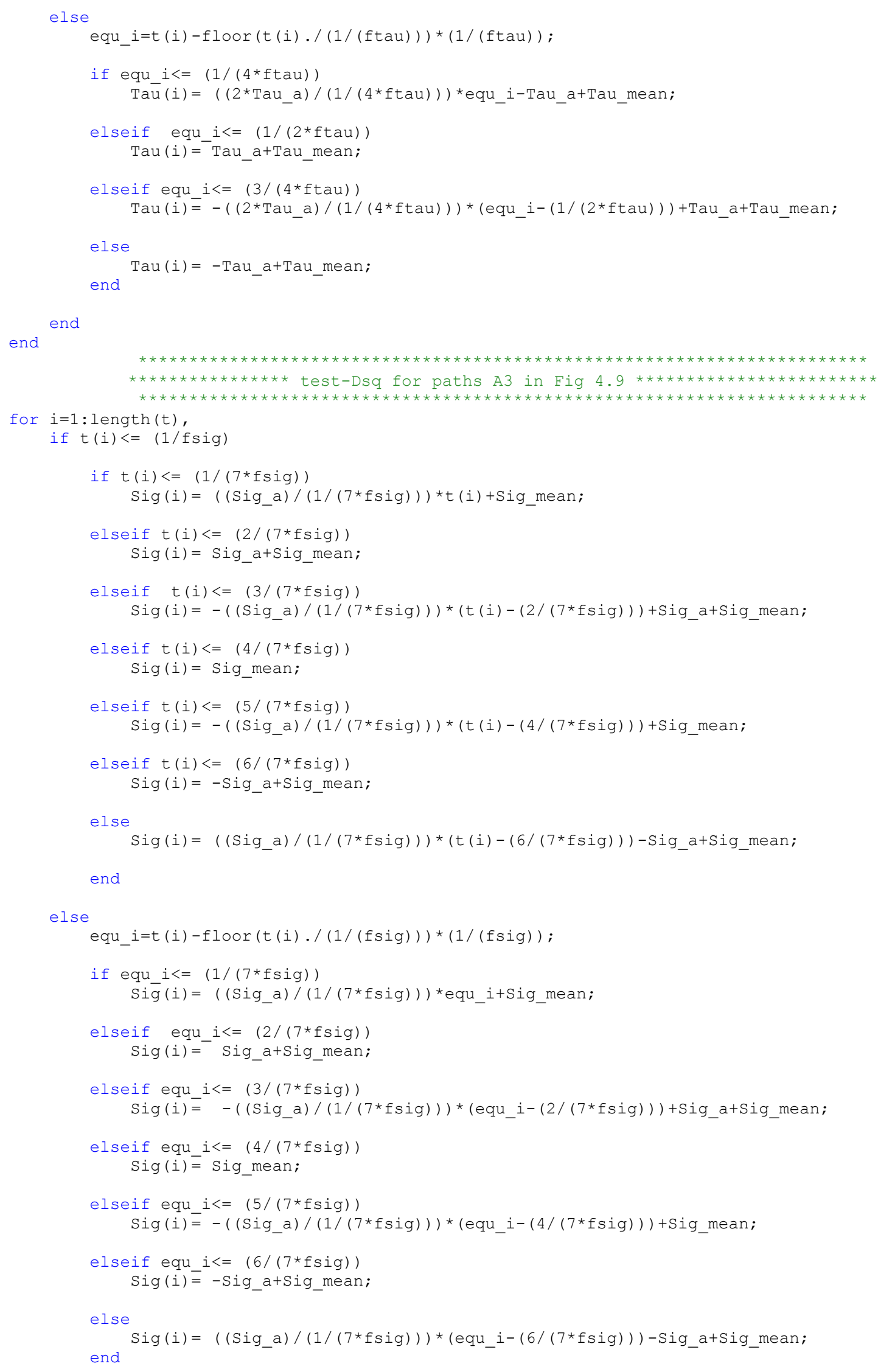




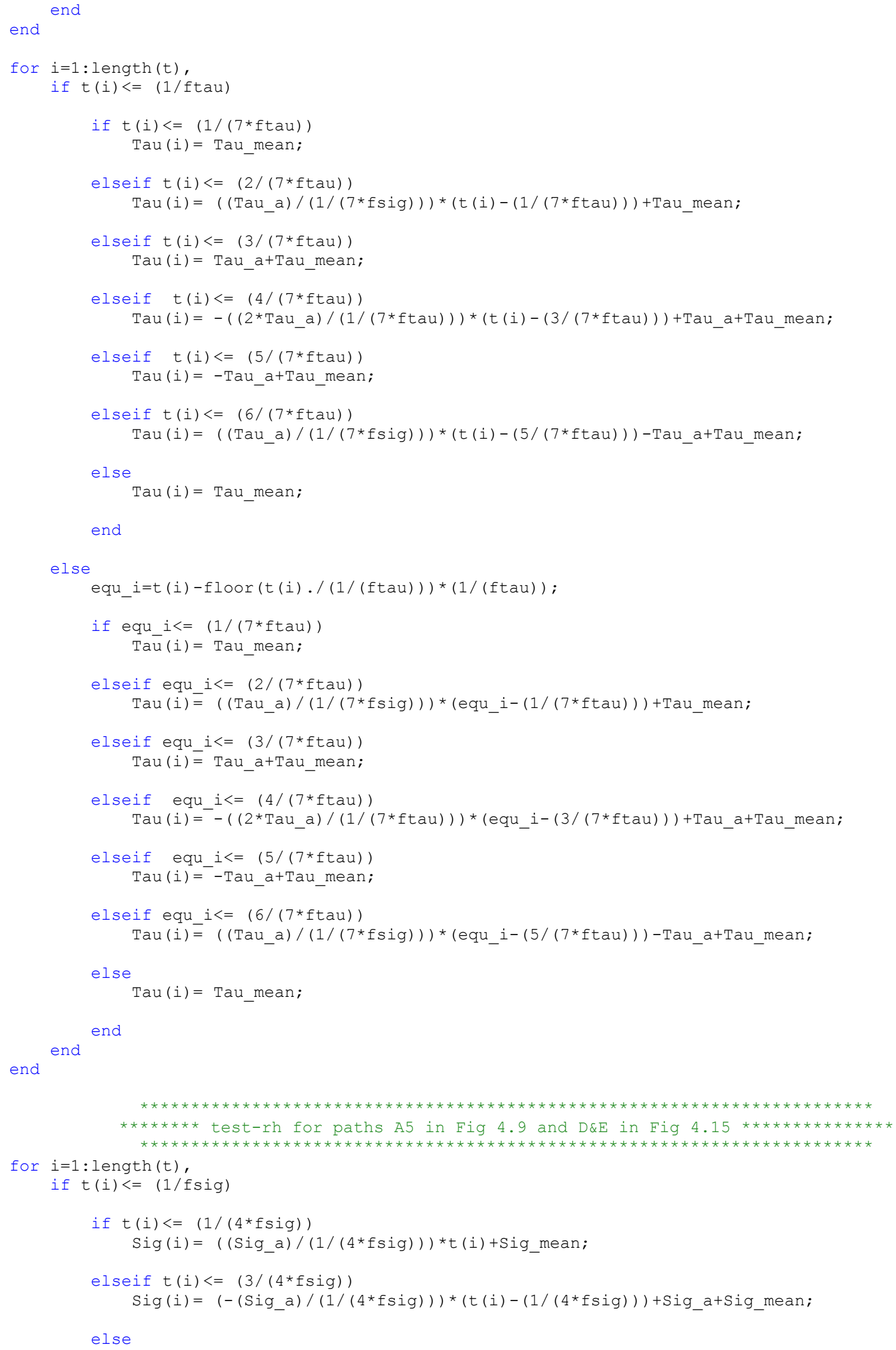




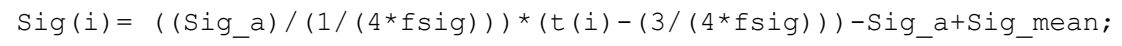

end

else

equ_i=t(i) -floor (t (i)./(1/(fsig)))*(1/(fsig)) ;

if equ $i<=(1 /(4 *$ fsig) $)$

$\operatorname{sig}(i)=(($ Sig_a $) /(1 /(4 *$ fsig $)))$ *equ_i+Sig_mean;

elseif equ_i<=(3/(4*fsig))

$\operatorname{Sig}(i)=\left(-\left(\operatorname{Sig}_{-}\right) /(1 /(4 * \mathrm{fsig}))\right) *($ equ_i- $(1 /(4 * \mathrm{fsig})))+$ Sig_a+Sig_mean;

else

$\operatorname{Sig}(i)=\left(\left(\operatorname{Sig} \_\right) /(1 /(4 * f \operatorname{sig}))\right) *($ equ_i- $(3 /(4 * f \operatorname{sig})))-$ Sig_a+Sig_mean;

end

end

end

for $i=1:$ length $(t)$,

if $\mathrm{t}(\mathrm{i})<=(1 / \mathrm{ftau})$

if $t(i)<=(1 /(2 *$ ftau $))$

$\operatorname{Tau}(i)=(($ Tau_a $) /(1 /(4 * f s i g))) * t(i)-T a u \_a+T a u \_m e a n ;$

else

$\operatorname{Tau}(i)=(-($ Tau_a $) /(1 /(4 * f s i g))) *(t(i)-(2 /(4 * f t a u)))+T a u \_a+T a u \_m e a n ;$

end

else

equ_i=t(i) $-f \operatorname{loor}(t(i) . /(1 /($ ftau $))) *(1 /($ ftau $))$;

if equ_i<= (1/(2*ftau))

$\operatorname{Tau}(i)=(($ Tau_a $) /(1 /(4 *$ fsig) $)) *$ equ_i-Tau_a+Tau_mean;

else

$\operatorname{Tau}(i)=(-($ Tau_a $) /(1 /(4 * f s i g))) *($ equ_i-(2/(4*ftau $)))+T a u \_a+T a u \_m e a n ;$

end

end

axes (axesh)

plot (t,Sig, '.-k', t, Tau, '.-r')

xlabel ('Time, s')

ylabel('\sigma(t), \tau(t), MPa')

legend ('\sigma(t)', '\tau(t)')

axis tight

set (bhslow, 'Enable', 'on' )

$\operatorname{smax}=\max (\mathrm{abs}(\mathrm{Sig}))$;

end

function buttonslow (hobject, eventdata)

T=str2double (get (edithL, 'String')) ;

T0=str2double (get (edithSlow, 'String')) ;

fs=str2double (get (ediths, 'string')) ;

if $\mathrm{TO}>\mathrm{T}$,

'Warning ') ;

$f=$ warndlg('Time of slow start cannot be longer than time of stress signals.', $\mathrm{T} 0=\mathrm{T} ;$

set (edithslow, 'string', num2str(T0))

uiwait(f)

end

$\mathrm{nr}=\mathrm{TO}$ * $\mathrm{fs}$;

$\mathrm{X}=[$ Sig' Tau'] ;

$\mathrm{w}=\sin \left([0: \mathrm{pi} / 2 / \mathrm{nr}: \mathrm{pi} / 2]^{\prime}\right)$;

$\mathrm{W}=\mathrm{W}^{*}$ ones $(1, \operatorname{size}(\mathrm{X}, 2))$;

$\mathrm{X}(1: \operatorname{size}(\mathrm{w}, 1),:)=\mathrm{w} \cdot{ }^{\star} \mathrm{X}(1: \operatorname{size}(\mathrm{w}, 1),:)$; 


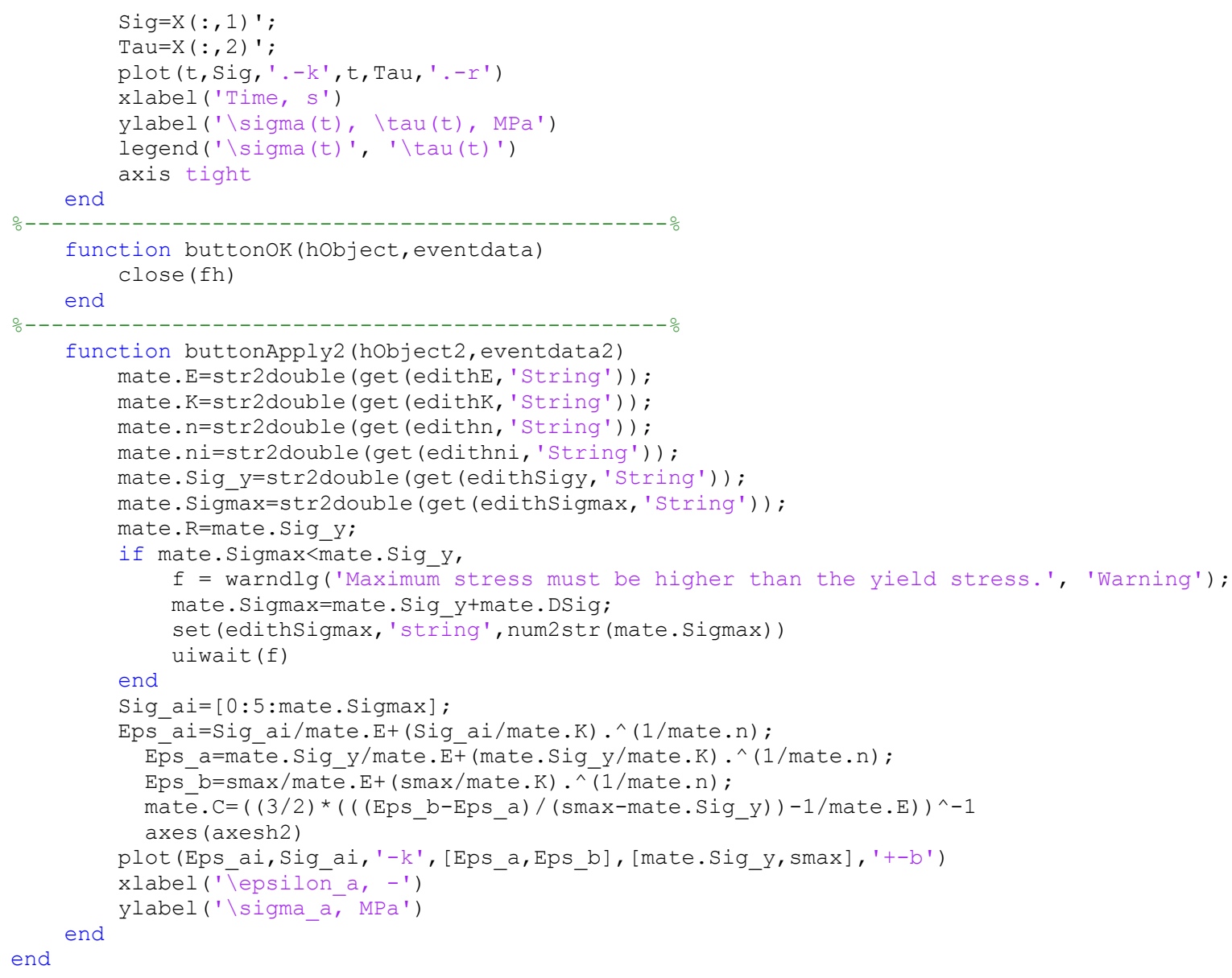




\section{APPENDIX B}

Experimental data employed in chapter four to assess the ratcheting response of steel alloys under various multiaxial loading spectra are presented in Appendix B. Table B.1 presents mechanical properties of steel alloys used in this thesis. Experimental ratcheting data for 1045 steel samples subjected to eight various loading paths were used to evaluate the capability of the modified hardening rule in ratcheting assessment under multiaxial loading cycles (see figure 4.4). These experimental data are presented in Tables B.2 and B.3. The capability of the O-W, J-S, McDowell and modified A-V hardening rules in the ratcheting prediction of 1045 steel and 1Cr18Ni9Ti stainless steel under different multiaxial loading histories was evaluated in figures 4.7 and 4.8. Experimental ratcheting data in these figures are tabulated in Tables B.3 and B.4.

Ratcheting data of SS304 steel samples were compared with predicted curves based on the hardening rules of Ohno-Wang $(\mathrm{O}-\mathrm{W})$, Chen-Jiao-Kim $(\mathrm{C}-\mathrm{J}-\mathrm{K})$ and the modified hardening rule under both stress-controlled and mixed stress- and strain-controlled multiaxial histories as presented in figures 4.13 and 4.14. Tables B.5, B.6 and B.7 present experimental ratcheting strain data for multiaxial loading paths A1-A5 and B1-B6 as presented in figure 4.13-4.14. The hardening rules of Ohno-Wang (O-W), Chen-Jiao-Kim (C-J-K) and the modified model were evaluated for their capability in ratcheting assessment of as compared with SS304 ratcheting data reported for various multiaxial step-loading histories presented in figures 4.17-4.22. The experimental ratcheting data for these multiaxial paths are presented in Table B.8. 
Table B.1 Mechanical properties of steel alloys studied in this thesis.

\begin{tabular}{ccccc}
\hline Material & $\boldsymbol{\sigma}_{\mathbf{y}}(\mathbf{M P a})$ & $\mathbf{E}(\mathbf{G P a})$ & $\mathbf{G}(\mathbf{G P a})$ & $\boldsymbol{\sigma}_{\mathbf{u}}(\mathbf{M P a})$ \\
\hline 1045 steel [30] & 590 & 205 & 79 & 798 \\
\hline 1Cr18Ni9Ti [29] & 310 & 193 & 65.5 & 605 \\
\hline SS304L [47] & 19 & 205 & 80 & 480 \\
\hline SS304 [50] & 210 & 192 & 83 & $500-510$ \\
\hline SS304 [51] & 230 & 195 & 86 & $500-510$ \\
\hline
\end{tabular}


Table B.2 Uniaxial experimental ratcheting data of 1045 steel alloy [30] used to determine calibrating coefficient $\gamma_{2}$ in figure 4.3.

\begin{tabular}{c|c}
\multicolumn{2}{c}{ Uniaxial $(\mathbf{1 0 0} \pm \mathbf{3 7 0 M P a})$} \\
\hline Cycles $(\mathbf{N})$ & $\boldsymbol{\varepsilon}_{\mathbf{r}}(\mathbf{\%})$ \\
\hline 0 & 0.00 \\
1 & 0.38 \\
40 & 0.81 \\
100 & 1.19 \\
200 & 1.62 \\
300 & 1.93
\end{tabular}


Table B.3 Multiaxial ratcheting data of 1045 steel alloy [30] for various loading paths A-H used in figures 4.4 and 4.7.

Path A

\begin{tabular}{c|c}
\hline Cycles $(\mathbf{N})$ & $\boldsymbol{\varepsilon}_{\mathbf{r}}(\mathbf{\%})$ \\
\hline 0 & 0.00 \\
6 & 0.30 \\
24 & 0.58 \\
60 & 0.96 \\
80 & 1.09 \\
94 & 1.17 \\
140 & 1.42 \\
210 & 1.71 \\
230 & 1.76 \\
240 & 1.81 \\
255 & 1.84 \\
270 & 1.88 \\
280 & 1.92 \\
300 & 1.96 \\
\end{tabular}

Path B

\begin{tabular}{c|c}
\hline Cycles $(\mathbf{N})$ & $\boldsymbol{\varepsilon}_{\mathrm{r}}(\mathbf{\%})$ \\
\hline 0 & 0.00 \\
9 & 0.34 \\
20 & 0.41 \\
30 & 0.49 \\
40 & 0.54 \\
50 & 0.59 \\
60 & 0.62 \\
70 & 0.68 \\
80 & 0.69 \\
90 & 0.70 \\
100 & 0.74 \\
200 & 0.97 \\
300 & 1.11 \\
--- & ---
\end{tabular}

Path C

\begin{tabular}{|c|c|}
\hline Cycles (N) & $\varepsilon_{\mathrm{r}}(\%)$ \\
\hline 0 & 0.00 \\
\hline 19 & 0.36 \\
\hline 30 & 0.40 \\
\hline 40 & 0.46 \\
\hline 50 & 0.51 \\
\hline 60 & 0.57 \\
\hline 70 & 0.59 \\
\hline 80 & 0.64 \\
\hline 90 & 0.67 \\
\hline 100 & 0.68 \\
\hline 200 & 0.92 \\
\hline 300 & 1.07 \\
\hline --- & --- \\
\hline --- & --- \\
\hline
\end{tabular}

Path D

\begin{tabular}{c|c}
\hline Cycles $(\mathbf{N})$ & $\boldsymbol{\varepsilon}_{\mathbf{r}}(\boldsymbol{\%})$ \\
\hline 0 & 0.23 \\
19 & 0.49 \\
40 & 0.63 \\
60 & 0.71 \\
80 & 0.75 \\
100 & 0.82 \\
120 & 0.88 \\
140 & 0.92 \\
160 & 0.98 \\
180 & 1.02 \\
200 & 1.04 \\
300 & 1.17 \\
--- & --- \\
--- & ---
\end{tabular}


Table B.3 Continued

Path E

\begin{tabular}{c|c}
\hline Cycles $(\mathbf{N})$ & $\boldsymbol{\varepsilon}_{\mathbf{r}}(\mathbf{\%})$ \\
\hline 0 & 0.00 \\
2 & 0.16 \\
3 & 0.24 \\
4 & 0.30 \\
8 & 0.36 \\
10 & 0.39 \\
20 & 0.50 \\
30 & 0.58 \\
40 & 0.65 \\
50 & 0.70 \\
60 & 0.75 \\
70 & 0.80 \\
80 & 0.84 \\
90 & 0.89 \\
100 & 0.92 \\
200 & 1.21 \\
300 & 1.40 \\
--- & --- \\
&
\end{tabular}

Path F

\begin{tabular}{c|c}
\hline Cycles $(\mathbf{N})$ & $\boldsymbol{\varepsilon}_{\mathbf{r}}(\mathbf{\%})$ \\
\hline 0 & 0.00 \\
3 & 0.02 \\
4 & 0.04 \\
7 & 0.07 \\
10 & 0.09 \\
20 & 0.12 \\
30 & 0.15 \\
40 & 0.18 \\
50 & 0.21 \\
60 & 0.24 \\
70 & 0.26 \\
80 & 0.27 \\
90 & 0.28 \\
100 & 0.30 \\
200 & 0.42 \\
300 & 0.50 \\
--- & --- \\
--- & --- \\
\end{tabular}

Path G

\begin{tabular}{c|c}
\hline Cycles $(\mathbf{N})$ & $\boldsymbol{\varepsilon}_{\mathbf{r}}(\mathbf{\%})$ \\
\hline 0 & 0.00 \\
2 & 0.31 \\
3 & 0.44 \\
4 & 0.53 \\
6 & 0.61 \\
10 & 0.69 \\
11 & 0.76 \\
20 & 0.98 \\
30 & 1.15 \\
40 & 1.28 \\
50 & 1.40 \\
60 & 1.51 \\
70 & 1.60 \\
80 & 1.68 \\
90 & 1.78 \\
100 & 1.85 \\
200 & 2.44 \\
300 & 2.87 \\
\end{tabular}

Path H

\begin{tabular}{c|c}
\hline Cycles $(\mathbf{N})$ & $\boldsymbol{\varepsilon}_{\mathbf{r}}(\mathbf{\%})$ \\
\hline 0 & 0.00 \\
7 & 0.55 \\
9 & 0.63 \\
10 & 0.70 \\
20 & 0.96 \\
30 & 1.16 \\
40 & 1.31 \\
50 & 1.43 \\
60 & 1.53 \\
70 & 1.63 \\
80 & 1.73 \\
90 & 1.78 \\
100 & 1.86 \\
200 & 2.38 \\
300 & 2.75 \\
--- & --- \\
--- & --- \\
--- & --- \\
\end{tabular}


Table B.4 Multiaxial ratcheting data of 1Cr18Ni9Ti steel alloy [29] for loading paths I-K used in figure 4.8 .

\begin{tabular}{|c|c|c|c|c|c|}
\hline \multicolumn{2}{|c|}{ Path I } & \multicolumn{2}{|c|}{ Path J } & \multicolumn{2}{|c|}{ Path K } \\
\hline Cycles (N) & $\varepsilon_{\mathrm{r}}(\%)$ & Cycles (N) & $\varepsilon_{r}(\%)$ & Cycles (N) & $\varepsilon_{r}(\%)$ \\
\hline 0 & 0.00 & 0 & 0.00 & 0 & 0.00 \\
\hline 1 & 0.18 & 1 & 0.30 & 1 & 0.48 \\
\hline 2 & 0.26 & 2 & 0.34 & 2 & 0.54 \\
\hline 3 & 0.30 & 3 & 0.37 & 3 & 0.59 \\
\hline 4 & 0.32 & 4 & 0.39 & 4 & 0.62 \\
\hline 5 & 0.35 & 5 & 0.41 & 5 & 0.65 \\
\hline 5 & 0.37 & 6 & 0.42 & 6 & 0.68 \\
\hline 7 & 0.39 & 7 & 0.43 & 7 & 0.71 \\
\hline 9 & 0.40 & 8 & 0.44 & 8 & 0.73 \\
\hline 10 & 0.42 & 9 & 0.45 & 9 & 0.75 \\
\hline 15 & 0.45 & 10 & 0.46 & 10 & 0.78 \\
\hline 31 & 0.51 & 16 & 0.50 & 20 & 0.93 \\
\hline 63 & 0.57 & 32 & 0.56 & 40 & 1.11 \\
\hline--- & --- & 64 & 0.62 & 60 & 1.22 \\
\hline
\end{tabular}


Table B.5 Uniaxial experimental ratcheting data of SS304 steel alloys [47, 50-51] used to determine calibrating coefficient $\gamma_{2}$ in figure 4.12

SS304L [47]

Uniaxial Loading 50 \pm 200

\begin{tabular}{|c|c|}
\hline Cycles (N) & $\varepsilon_{\mathrm{r}}(\%)$ \\
\hline 0 & 0.00 \\
\hline 1 & 1.03 \\
\hline 6 & 1.35 \\
\hline 11 & 1.49 \\
\hline 16 & 1.58 \\
\hline 21 & 1.66 \\
\hline 26 & 1.72 \\
\hline 31 & 1.78 \\
\hline 36 & 1.83 \\
\hline 41 & 1.87 \\
\hline 46 & 1.91 \\
\hline 51 & 1.95 \\
\hline 56 & 1.98 \\
\hline 61 & 2.01 \\
\hline 66 & 2.04 \\
\hline 71 & 2.07 \\
\hline 76 & 2.10 \\
\hline 81 & 2.12 \\
\hline 86 & 2.14 \\
\hline 91 & 2.17 \\
\hline 96 & 2.19 \\
\hline
\end{tabular}

SS304 [50]

Uniaxial Loading $78 \pm 248$

\begin{tabular}{c|c}
\hline Cycles $(\mathbf{N})$ & $\boldsymbol{\varepsilon}_{\mathbf{r}}(\%)$ \\
\hline 0 & 0.00 \\
1 & 0.89
\end{tabular}

3

6

9

13

19

25

30

36

43

50

$--$

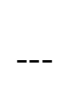

---

$---$

$-$

$--$

$--$

$---$

$--$
SS304 [51]

Uniaxial Loading 235 \pm 235

\begin{tabular}{c|c}
\hline Cycles $(\mathbf{N})$ & $\boldsymbol{\varepsilon}_{\mathbf{r}}(\mathbf{\%})$ \\
\hline 0 & 0.00 \\
4 & 1.18 \\
15 & 1.43 \\
39 & 1.66 \\
79 & 1.84 \\
134 & 1.97 \\
216 & 2.15 \\
300 & 2.28
\end{tabular}


Table B.6 Multiaxial ratcheting data for SS304 alloys [47, 50] for loading paths A1-A5 under stress-controlled loading conditions used in figure 4.13.

\begin{tabular}{|c|c|c|c|c|c|}
\hline \multicolumn{2}{|c|}{ Path A1 [47] } & \multicolumn{2}{|c|}{ Path A2 [47] } & \multicolumn{2}{|c|}{ Path A3 [47] } \\
\hline Cycles (N) & $\varepsilon_{\mathrm{r}}(\%)$ & Cycles (N) & $\varepsilon_{\mathrm{r}}(\%)$ & Cycles (N) & $\varepsilon_{\mathrm{r}}(\%)$ \\
\hline 0 & 0.00 & 0 & 0.00 & 0 & 0.00 \\
\hline 1 & 0.035 & 1 & 0.194 & 1 & 0.391 \\
\hline 6 & 0.063 & 6 & 0.261 & 6 & 0.444 \\
\hline 11 & 0.063 & 11 & 0.274 & 11 & 0.472 \\
\hline 16 & 0.070 & 16 & 0.285 & 16 & 0.493 \\
\hline 21 & 0.070 & 21 & 0.292 & 21 & 0.514 \\
\hline 26 & 0.072 & 26 & 0.297 & 26 & 0.526 \\
\hline 31 & 0.075 & 31 & 0.306 & 31 & 0.540 \\
\hline 36 & 0.075 & 36 & 0.308 & 36 & 0.554 \\
\hline 41 & 0.075 & 41 & 0.313 & 41 & 0.566 \\
\hline 46 & 0.077 & 46 & 0.320 & 46 & 0.578 \\
\hline 51 & 0.080 & 51 & 0.323 & 51 & 0.592 \\
\hline 56 & 0.077 & 56 & 0.325 & 56 & 0.599 \\
\hline 61 & 0.080 & 61 & 0.330 & 61 & 0.610 \\
\hline 66 & 0.082 & 66 & 0.335 & 66 & 0.619 \\
\hline 71 & 0.080 & 71 & 0.337 & 71 & 0.627 \\
\hline 76 & 0.077 & 76 & 0.337 & 76 & 0.639 \\
\hline 81 & 0.082 & 81 & 0.341 & 81 & 0.645 \\
\hline 86 & 0.082 & 86 & 0.346 & 86 & 0.655 \\
\hline 91 & 0.084 & 91 & 0.348 & 91 & 0.662 \\
\hline 96 & 0.084 & 96 & 0.348 & 96 & 0.673 \\
\hline
\end{tabular}


Table B.6 Continued

\begin{tabular}{c|c}
\multicolumn{2}{c}{ Path A4 [50] } \\
\hline Cycles $(\mathbf{N})$ & $\boldsymbol{\varepsilon}_{\mathbf{r}}(\mathbf{\%})$ \\
\hline 0 & 0.00 \\
1 & 0.40 \\
2 & 0.635 \\
3 & 0.694 \\
4 & 0.724 \\
5 & 0.742 \\
6 & 0.756 \\
7 & 0.764 \\
9 & 0.780 \\
11 & 0.790 \\
13 & 0.800 \\
15 & 0.812 \\
17 & 0.817 \\
19 & 0.828 \\
21 & 0.832 \\
23 & 0.839 \\
25 & 0.843 \\
27 & 0.848 \\
29 & 0.856 \\
31 & 0.860 \\
33 & 0.860 \\
35 & 0.866 \\
37 & 0.870 \\
39 & 0.876 \\
41 & 0.879 \\
43 & 0.880 \\
45 & 0.880 \\
47 & 0.884 \\
50 & 0.888 \\
--- & --- \\
-- & --- \\
\hline
\end{tabular}

\begin{tabular}{c|c}
\multicolumn{2}{c}{ Path A5 [50] } \\
\hline Cycles $\mathbf{( N )}$ & $\boldsymbol{\varepsilon}_{\mathbf{r}} \mathbf{( \% )}$ \\
\hline 0 & 0.00 \\
1 & 0.40 \\
2 & 0.431 \\
3 & 0.453 \\
4 & 0.463 \\
5 & 0.467 \\
6 & 0.479 \\
7 & 0.491 \\
9 & 0.495 \\
11 & 0.503 \\
13 & 0.511 \\
15 & 0.515 \\
17 & 0.521 \\
19 & 0.525 \\
20 & 0.528 \\
23 & 0.531 \\
25 & 0.539 \\
27 & 0.543 \\
29 & 0.546 \\
31 & 0.547 \\
32 & 0.547 \\
34 & 0.551 \\
36 & 0.553 \\
38 & 0.555 \\
40 & 0.560 \\
42 & 0.564 \\
44 & 0.568 \\
45 & 0.567 \\
47 & 0.567 \\
\hline & 0.571 \\
\hline & 0.571 \\
\hline
\end{tabular}


Table B.7 Multiaxial ratcheting data for SS304 alloys [47, 51] for loading paths B1-B6 under mixed strain- and stress-controlled conditions used in figure 4.14.

\section{Path B1 [51]}

\begin{tabular}{c|c}
\hline Cycles $(\mathbf{N})$ & $\boldsymbol{\varepsilon}_{\mathbf{r}}(\mathbf{\%})$ \\
\hline 0 & 0.00 \\
1 & 0.25 \\
2 & 0.40 \\
3 & 0.59 \\
5 & 0.75 \\
12 & 0.87 \\
20 & 0.97 \\
30 & 1.06 \\
44 & 1.15 \\
59 & 1.23 \\
72 & 1.28 \\
84 & 1.32 \\
101 & 1.37 \\
113 & 1.41 \\
128 & 1.45 \\
142 & 1.50 \\
163 & 1.56 \\
191 & 1.62 \\
213 & 1.67 \\
234 & 1.72 \\
256 & 1.76 \\
282 & 1.83 \\
300 & 1.87 \\
--- & --- \\
--- & --- \\
--- & --- \\
&
\end{tabular}

Path B2 [51]

\begin{tabular}{c|c}
\hline Cycles $(\mathbf{N})$ & $\boldsymbol{\varepsilon}_{\mathbf{r}}(\mathbf{\%})$ \\
\hline 0 & 0.00 \\
2 & 0.18 \\
3 & 0.24 \\
4 & 0.30 \\
6 & 0.38 \\
12 & 0.42 \\
21 & 0.47 \\
33 & 0.50 \\
44 & 0.53 \\
60 & 0.55 \\
73 & 0.57 \\
87 & 0.59 \\
105 & 0.61 \\
128 & 0.63 \\
151 & 0.66 \\
176 & 0.68 \\
213 & 0.72 \\
245 & 0.74 \\
274 & 0.77 \\
300 & 0.80 \\
--- & --- \\
--- & --- \\
--- & --- \\
--- & --- \\
--- & --- \\
--- & --- \\
&
\end{tabular}

Path B3 [51]

\begin{tabular}{c|c}
\hline Cycles $(\mathbf{N})$ & $\boldsymbol{\varepsilon}_{\mathbf{r}}(\mathbf{\%})$ \\
\hline 0 & 0.00 \\
1 & 0.26 \\
2 & 0.30 \\
3 & 0.35 \\
5 & 0.40 \\
8 & 0.44 \\
14 & 0.47 \\
21 & 0.49 \\
33 & 0.51 \\
43 & 0.53 \\
54 & 0.53 \\
68 & 0.55 \\
83 & 0.56 \\
95 & 0.57 \\
112 & 0.58 \\
126 & 0.59 \\
142 & 0.59 \\
158 & 0.60 \\
175 & 0.61 \\
191 & 0.62 \\
208 & 0.63 \\
226 & 0.63 \\
243 & 0.64 \\
261 & 0.65 \\
279 & 0.66 \\
300 & 0.66 \\
&
\end{tabular}


Table B.7 Continued

\begin{tabular}{c|ccccccc}
\multicolumn{2}{c}{ Path B4 [51] } & & \multicolumn{2}{c}{ Path B5 [51] } & & \multicolumn{2}{c}{ Path B6 [47] } \\
\cline { 1 - 4 } \cline { 6 - 7 } Cycles $(\mathbf{N})$ & $\boldsymbol{\varepsilon}_{\mathbf{r}}(\mathbf{\%})$ & & Cycles $(\mathbf{N})$ & $\boldsymbol{\varepsilon}_{\mathbf{r}}(\mathbf{\%})$ & & Cycles $(\mathbf{N})$ & $\boldsymbol{\varepsilon}_{\mathbf{r}}(\mathbf{\%})$ \\
\hline 0 & 0.00 & & 0 & 0.00 & & 0 & 0.00 \\
1 & 0.16 & & 1 & 0.18 & & 1 & 1.64 \\
2 & 0.28 & & 2 & 0.23 & & 6 & 2.68 \\
3 & 0.43 & & 3 & 0.29 & & 11 & 2.99 \\
5 & 0.61 & & 10 & 0.35 & & 16 & 3.13 \\
11 & 0.82 & & 23 & 0.40 & & 21 & 3.21 \\
17 & 0.99 & & 40 & 0.43 & & 26 & 3.26 \\
28 & 1.21 & & 54 & 0.44 & & 31 & 3.30 \\
43 & 1.39 & & 74 & 0.46 & & 36 & 3.32 \\
61 & 1.57 & & 94 & 0.47 & & 41 & 3.35 \\
83 & 1.74 & & 112 & 0.48 & & 46 & 3.38 \\
106 & 1.88 & & 138 & 0.50 & & 51 & 3.40 \\
138 & 2.04 & & 165 & 0.51 & & 56 & 3.42 \\
170 & 2.19 & & 192 & 0.52 & & 61 & 3.44 \\
200 & 2.34 & & 232 & 0.54 & & 66 & 3.46 \\
229 & 2.44 & & 269 & 0.56 & & 71 & 3.48 \\
253 & 2.52 & & 300 & 0.57 & & 76 & 3.49 \\
280 & 2.61 & & --- & --- & & 81 & 3.52 \\
300 & 2.68 & & --- & --- & & 86 & 3.53 \\
--- & --- & & --- & --- & & 91 & 3.54 \\
--- & --- & & --- & --- & & 96 & 3.56 \\
& & & & & &
\end{tabular}


Table B.8 Ratcheting data for SS304 alloys [43, 50] for loading paths A-E under various multiaxial step-loading spectra used in figure 4.17-4-22.

Path A

\begin{tabular}{c|ccc|c}
\hline Cycles $(\mathbf{N})$ & $\boldsymbol{\varepsilon}_{\mathbf{r}}(\mathbf{\%})$ & & Cycles $(\mathbf{N})$ & $\boldsymbol{\varepsilon}_{\mathbf{r}}(\mathbf{\%})$ \\
\hline 0 & 0.00 & & 63 & 2.31 \\
3 & 0.86 & & 65 & 2.35 \\
5 & 0.91 & & 67 & 2.39 \\
7 & 0.94 & & 70 & 2.44 \\
9 & 0.96 & & 74 & 2.51 \\
11 & 0.98 & & 78 & 2.56 \\
13 & 1.00 & & 82 & 2.60 \\
17 & 1.02 & & 86 & 2.64 \\
20 & 1.03 & & 90 & 2.67 \\
24 & 1.06 & & 94 & 2.71 \\
28 & 1.08 & & 98 & 2.73 \\
32 & 1.09 & & 101 & 2.71 \\
36 & 1.11 & & 103 & 2.71 \\
40 & 1.12 & & 106 & 2.71 \\
43 & 1.13 & & 110 & 2.71 \\
47 & 1.14 & & 114 & 2.71 \\
49 & 1.15 & & 118 & 2.71 \\
51 & 1.52 & --- & --- \\
53 & 1.84 & --- & --- \\
55 & 1.99 & --- & --- \\
57 & 2.09 & --- & --- \\
59 & 2.17 & & --- & --- \\
61 & 2.24 & & --- & --- \\
& & & &
\end{tabular}

Path B

\begin{tabular}{c|ccc|c}
\hline Cycles $(\mathbf{N})$ & $\boldsymbol{\varepsilon}_{\mathbf{r}}(\mathbf{\%})$ & & Cycles $(\mathbf{N})$ & $\boldsymbol{\varepsilon}_{\mathbf{r}}(\mathbf{\%})$ \\
\hline 0 & 0.00 & & 68 & 1.88 \\
1 & 0.40 & & 70 & 1.91 \\
3 & 0.69 & & 72 & 1.93 \\
5 & 0.74 & & 75 & 1.96 \\
7 & 0.76 & & 78 & 1.99 \\
11 & 0.79 & & 80 & 2.02 \\
15 & 0.81 & & 83 & 2.05 \\
19 & 0.83 & & 85 & 2.06 \\
23 & 0.84 & & 88 & 2.08 \\
27 & 0.85 & & 91 & 2.11 \\
31 & 0.86 & & 93 & 2.13 \\
35 & 0.87 & & 96 & 2.15 \\
39 & 0.88 & & 98 & 2.16 \\
43 & 0.88 & & 100 & 2.19 \\
47 & 0.88 & & 103 & 2.13 \\
51 & 1.06 & & 106 & 2.13 \\
54 & 1.51 & & 108 & 2.13 \\
56 & 1.61 & & 111 & 2.13 \\
58 & 1.68 & & 113 & 2.13 \\
60 & 1.74 & & 115 & 2.13 \\
62 & 1.78 & & 117 & 2.13 \\
64 & 1.82 & & 119 & 2.13 \\
66 & 1.86 & & -- & --- \\
& & & &
\end{tabular}


Table B.8 Continued

Path C

\begin{tabular}{c|ccc|c}
\hline Cycles $(\mathbf{N})$ & $\boldsymbol{\varepsilon}_{\mathbf{r}}(\mathbf{\%})$ & & Cycles $(\mathbf{N})$ & $\boldsymbol{\varepsilon}_{\mathbf{r}}(\mathbf{\%})$ \\
\hline 0 & 0.00 & & 53 & 1.55 \\
2 & 0.52 & & 55 & 1.65 \\
3 & 0.58 & & 57 & 1.72 \\
5 & 0.62 & & 60 & 1.79 \\
7 & 0.65 & & 63 & 1.85 \\
9 & 0.67 & & 67 & 1.91 \\
12 & 0.70 & & 71 & 1.96 \\
14 & 0.71 & & 75 & 2.01 \\
17 & 0.73 & & 80 & 2.05 \\
20 & 0.74 & & 85 & 2.10 \\
22 & 0.76 & & 88 & 2.12 \\
26 & 0.77 & & 91 & 2.15 \\
28 & 0.78 & & 95 & 2.18 \\
31 & 0.79 & & 98 & 2.20 \\
34 & 0.80 & & 100 & 2.21 \\
37 & 0.80 & & 102 & 2.17 \\
40 & 0.81 & & 103 & 2.16 \\
43 & 0.82 & & 105 & 2.16 \\
46 & 0.83 & & 108 & 2.16 \\
50 & 0.83 & & 111 & 2.16 \\
51 & 1.11 & & 115 & 2.16 \\
52 & 1.30 & & 118 & 2.17 \\
52 & 1.45 & & 120 & 2.16 \\
& & & &
\end{tabular}

Path D

\begin{tabular}{c|ccc|c}
\hline Cycles $(\mathbf{N})$ & Er(\%) & & Cycles $(\mathbf{N})$ & $\boldsymbol{\varepsilon}_{\mathbf{r}}(\mathbf{\%})$ \\
\hline 0 & 0.00 & & 62 & 1.22 \\
1 & 0.36 & & 64 & 1.24 \\
3 & 0.45 & & 66 & 1.26 \\
5 & 0.47 & & 68 & 1.28 \\
7 & 0.49 & & 70 & 1.30 \\
9 & 0.49 & & 72 & 1.31 \\
11 & 0.50 & & 74 & 1.32 \\
13 & 0.51 & & 76 & 1.34 \\
15 & 0.52 & & 78 & 1.35 \\
17 & 0.52 & & 80 & 1.36 \\
21 & 0.53 & & 82 & 1.37 \\
25 & 0.54 & & 85 & 1.38 \\
29 & 0.55 & & 88 & 1.40 \\
37 & 0.56 & & 92 & 1.42 \\
41 & 0.56 & & 96 & 1.44 \\
50 & 0.57 & & 100 & 1.45 \\
51 & 0.85 & 103 & 1.41 \\
52 & 0.97 & 107 & 1.41 \\
53 & 1.03 & 111 & 1.41 \\
54 & 1.07 & 118 & 1.40 \\
56 & 1.12 & --- & --- \\
58 & 1.16 & --- & --- \\
60 & 1.19 & & --- & -- \\
& & & &
\end{tabular}


Table B.8 Continued

Path E

\begin{tabular}{c|cc|c|c}
\hline Cycles $(\mathbf{N})$ & $\boldsymbol{\varepsilon}_{\mathbf{r}}(\mathbf{\%})$ & Cycles $(\mathbf{N})$ & $\boldsymbol{\varepsilon}_{\mathbf{r}}(\mathbf{\%})$ \\
\hline 0 & 0.00 & & 52 & 1.68 \\
1 & 0.89 & & 54 & 1.81 \\
2 & 0.96 & & 56 & 1.91 \\
3 & 0.99 & & 59 & 1.99 \\
5 & 1.01 & & 62 & 2.05 \\
8 & 1.02 & & 64 & 2.08 \\
11 & 1.03 & & 68 & 2.14 \\
14 & 1.04 & & 71 & 2.18 \\
17 & 1.05 & & 76 & 2.23 \\
21 & 1.06 & & 79 & 2.26 \\
25 & 1.08 & & 83 & 2.30 \\
29 & 1.09 & & 86 & 2.32 \\
32 & 1.09 & & 90 & 2.35 \\
36 & 1.10 & & 93 & 2.37 \\
38 & 1.10 & & 96 & 2.39 \\
41 & 1.10 & & 100 & 2.42 \\
44 & 1.11 & & 101 & 2.39 \\
47 & 1.11 & 106 & 2.37 \\
50 & 1.12 & 109 & 2.37 \\
50 & 1.21 & 112 & 2.37 \\
51 & 1.30 & & 114 & 2.37 \\
51 & 1.42 & & 117 & 2.37 \\
51 & 1.54 & & 119 & 2.37 \\
& & & &
\end{tabular}




\section{REFERENCES}

[1] Varvani-Farahani, A., Brebbia, C.A. (2003). Fatigue Damage of Materials: Experiment and Analysis. Southampton: WIT Press.

[2] Bairstow, L. (1911). The Elastic Limits of Iron and Steel under Cyclical Variations of Stress, Philosophical Transactions of the Royal Society of London, Series A, 210, pp. 35-55.

[3] Chen, X., Gao, B. and Chen, G., (2006). Ratcheting study of pressurized elbows subjected to reversed in-plane bending. Journal of Pressure Vessel Technology, 128(4), pp. 525-532.

[4] Hassan, T., and Kyriakides, S. (1992), Ratcheting in cyclic plasticity, Part I: uniaxial behavior. International Journal of Plasticity, 8, pp. 91-116.

[5] Hassan, T., Corona, E., and Kyriakides, S., (1992), Ratchetting in cyclic plasticity part II: multiaxial behavior. International Journal of Plasticity, 8, pp. 117-146.

[6] Jiang, Y., (1993). Cyclic plasticity with emphasis on ratcheting. PhD thesis, University of Illinois at Urbana-Champaign, Champaign, IL.

[7] Hassan, T., and Kyriakides, S. (1994). Ratcheting of cyclically hardening and softening materials, Part I: uniaxial behavior. International Journal of Plasticity, 10, pp. 149-184.

[8] Hassan, T., and Kyriakides, S. (1994). Ratcheting of cyclically hardening and softening materials, Part II: multiaxial behavior. International Journal of Plasticity, 10, pp. 185-212.

[9] Kim, K.S., Jiao, R., Chen, X. and Sakane, M. (2009). Ratcheting of Stainless Steel 304 under Multiaxial Nonproportional Loading. Journal of Pressure Vessel Technology, 131(2), 021405

[10] Kang, G.Z., and Liu, Y.J. (2008). Uniaxial ratcheting and low-cycle fatigue failure of the steel with cyclic stabilizing or softening feature. Materials Science and Engineering: A, 472, pp. 258268.

[11] Taleb, L., and Hauet, A. (2009). Multiscale experimental investigations about the cyclic behavior of the 304L SS. International Journal of Plasticity, 25, pp. 1359-1385. 
[12] Yaguchi, M., and Takahashi, Y. (2005). Ratcheting of viscoplastic material with cyclic softening, part 1: experiments on modified 9Cr-1Mo steel. International Journal of Plasticity, 21, pp.43-65.

[13] Armstrong, P.J., and Fredrick, C.O. (1966). A mathematical representation of the multiaxial Bauschinger effect," CEGB Report RD/B/N731 Berkeley Nuclear Laboratories.

[14] Chaboche, J.L., (1991). On some modifications of kinematic hardening to improve the description of ratcheting effects. International Journal of Plasticity, 7, pp. 661-678.

[15] Bower, A.F. (1989). Cyclic hardening properties of hard-drawn copper and rail steel, Journal of the Mechanics and Physics of Solids, 37, 455-470.

[16] Bari, S., and Hassan, T. (2002). An advancement in cyclic plasticity modeling for multiaxial ratcheting simulation. International Journal of Plasticity, 7, pp. 873-894.

[17] Delobelle, P., Robinet, P., and Bocher, L. (1995). Experimental study and phenomenological modelization of ratcheting under uniaxial and biaxial loading on an austenitic stainless steel. International Journal of Plasticity, 11, pp. 295-330.

[18] Burlet, H., and Cailletaud, G. (1986). Numerical techniques for cyclic plasticity at variable temperature. Engineering Computations, 3, pp.143-153.

[19] Ohno, N. and Wang, J.D. (1993). Kinematic hardening rules with critical state of dynamic recovery, part I: formulations and basic features for ratcheting behavior. International Journal of Plasticity, 9, pp.375-390.

[20] Ohno, N., and Wang, J.D. (1993). Kinematic hardening rules with critical state of dynamic recovery, part II: application to experiments of ratcheting behavior. International Journal of Plasticity, 9, pp. 391-403.

[21] Jiang, Y., and Sehitoglu, H. (1996). Modeling of cyclic ratcheting plasticity, part I: development of constitutive relations," ASME Journal of Applied Mechanics, 63, pp. 720-725.

[22] Jiang, Y., and Sehitoglu, H., (1996). Modeling of cyclic ratcheting plasticity, part II: comparison of model simulations with experiments. ASME Journal of Applied Mechanics, 63, pp. 726-733. 
[23] McDowell, D.L., (1995). Stress state dependence of cyclic ratcheting behavior of two rail steels. International Journal of Plasticity, 11, pp. 397-421.

[24] Abdel-Karim, M., and Ohno, N. (1998). Uniaxial Ratchetting of 316FR Steel at Room Temperature-Part II: Constitutive Modeling and Simulation. J. Eng. Mater. Technol. 122(1):35-4. [25] Abdel-Karim, M., and Ohno, N. (2000). Kinematic hardening model suitable for ratcheting with steady-state. International Journal of Plasticity, 16, pp. 225-240.

[26] Döring, R., Hoffmeyer, J., Seeger, T. and Vormwald, M., (2003). A plasticity model for calculating stress-strain sequences under multiaxial nonproportional cyclic loading. Computational Materials Science, 28, pp. 587-596.

[27] Kang, G.Z., (2004). A viscoplastic constitutive model for ratcheting of cyclically stable materials and its finite element implementation," Mechanics of Materials 36 (4), pp. 299-312.

[28] Chen, X., Jiao, R. and Kim, K.S. (2003). Simulation of ratcheting strain to a high number of cycles under multiaxial loading. International Journal of Solids and Structures, 40, pp. 7449-7461.

[29] Chen, X., and Jiao, R. (2004). Modified kinematic hardening rule for multiaxial ratcheting prediction. International Journal of Plasticity, 20, pp. 871-898.

[30] Chen, X., Jiao, R., and Kim, K.S. (2005). On the Ohno-Wang kinematic hardening rules for multiaxial ratcheting modeling of medium carbon steel, International Journal of Plasticity, 6 , pp. 161-184.

[31] Yaguchi, M., and Takahashi, Y. (2005). "Ratcheting of viscoplastic material with cyclic softening, part 2: application of constitutive models. International Journal of Plasticity, 21, pp. 835-860.

[32] Colak, O.U. (2008). Kinematic hardening rules for modeling uniaxial and multiaxial ratcheting. Material \& Design, 29, pp. 1575-1581.

[33] Dafalias, Y.F. and Feigenbaum, H.P. (2011). Biaxial ratchetting with novel variations of kinematic hardening. International Journal of Plasticity, 27, pp. 479-491. 
[34] Ahmadzadeh, G.R., and Varvani-Farahani, A. (2013). Ratcheting assessment of materials based on the modified Armstrong-Frederick hardening rule at various uniaxial stress levels. Fatigue \& Fracture of Engineering Materials \& Structures, 36, pp. 1232-1245.

[35] Ahmadzadeh, G.R., and Varvani-Farahani, A. (2013). Ratcheting assessment of steel alloys under step-loading conditions. Material \& Design, 51, pp. 231-241.

[36] Bari, S., and Hassan, T. (2000). Anatomy of coupled constitutive models for ratcheting simulation. International Journal of Plasticity, 16, pp. 381-409.

[37] Dafalias, Y.F., Kourousis, K.I., and Saridis. G.J. (2008). Multiplicative AF kinematic hardening in plasticity. International Journal of Solids and Structures, 45, pp. 2861-2880.

[38] Dafalias, Y.F., Kourousis, K.I., and Saridis, G.J. (2008). Corrigendum to "Multiplicative AF kinematic hardening in plasticity," International Journal of Solids and Structures, 45, pp. 4878.

[39] Chaboche, J.L., Dang-Wan, K., and Cordier, G. (1979). Modelization of the strain memory effect on the cyclic hardening of 316 stainless steel. Proc. SMIRT-5. No. Division L., Berlin.

[40] Abdel-Karim M. (2009). Modified kinematic hardening rules for simulations of ratcheting. International Journal of Plasticity, 25, pp. 1560-1587.

[41] Tanaka, E. (1994). A nonproportional parameter and a cyclic viscoelastic constitutive model taking into account amplitude dependences and memory effects of isotropic hardening. Eur. J. Mech. A/Solids 13, 155-173.

[42] Aubin, V., Quaegebeur, P., Degallaix, S. (2003b). Cyclic plasticity of a duplex stainless steel under non-proportional loading. Material Science and Engineering A. 346, 208-215.

[43] Kang, G.Z., Gao, Q., Yang, X.J. (2004). Uniaxial and nonproportional multiaxial ratcheting of SS304 stainless steel at room temperature: Experiments and simulations, Int. J. Non-linear Mech. 39, 843-857.

[44] Benallal, A., Le Gallo, P., Marquis, D. (1989). An experimental investigation of cyclic hardening of 316 stainless steel and of 2024 aluminum alloy under multiaxial loading. Nucl. Eng. Des. 114, 345-353. 
[45] McDowell, D.L. (1985). A two surface model for transient nonproportional cyclic plasticity. ASME J. Appl. Mech. 52, 298-308.

[46] Jinghong Fan, Xianghe Peng. (1991). A physical based constitutive description for nonproportional cyclic plasticity. Trans. ASME, J Eng. Mater. Technol. 113(2), 254-262.

[47] Hassan, T., Taleb, L., Krishna, S. (2008). Influences of non-proportional loading paths on ratcheting responses and simulations by two recent cyclic plasticity models. Int. J. Plast. 24, 18631889.

[48] Corona, E., Hassan, T., Kyriakids, S., 1996. On the performance of kinematic hardening rules in predicting a class of biaxial ratchetting experiments. Int. J. Plast. 12 (1), 117-145.

[49] Hassan, T., Kyriakids, S., 1994. Ratchetting of cyclically hardening and softening materials: II. Multiaxial behavior. Int. J. Plast. 10 (2), 185-212.

[50] G. Kang, Q. Gao, L. Cai, Y. Sun, 2002. Experimental study on uniaxial and nonproportionally multiaxial ratcheting of SS304 stainless steel at room and high temperatures. Nuclear Engineering and Design 216, 13-26.

[51] Kim, K. S., Jiao, R. Chen, X., Sakane, M., 2009. Ratcheting of Stainless Steel 304 Under Multiaxial Nonproportional Loading. J. Pressure Vessel Technol. 131(2), 021405.

[52] Meggiolaro, M.A., de Castro, J.T.P. (2014). Prediction of non-proportionality factors of multiaxial histories using the moment of inertia method, Int. J. Fat. 61, 151-159.

[53] Jiang Y, Sehitoglu H. Multiaxial cyclic ratcheting under multiple step loading. Int J Plast. 10, $849-70$.

[54] Haupt A, Schinke B. (1996) Experiments of the ratcheting behavior of AISI 316L(N) austenitic steel at room temperature. J Eng Mater Technol. 118:281-4.

[55] Paul SK, Sivaprasad S, Dhar S, Tarafder S. (2011). Cyclic plastic deformation behavior in SA333 Gr. 6 C-Mn steel. Mater Sci Eng, A. 528:7341-9.

[56] Paul SK, Sivaprasad S, Dhar S, Tarafder S. (2012). True stress-controlled ratcheting behavior of 304LN stainless steel. J Mater Sci. 47:4660-72. 
[57] Kang GZ, Li Y, Gao Q, Kan QH, Zhang J. (2006) Uniaxial ratchetting in steels with different cyclic softening/hardening behaviours. Fatigue Fract Eng Mater Struct. 29:93-103.

[58] Kang GZ, Gao Q, Yang X. (2002). Experimental study on the cyclic deformation and plastic flow of U71Mn rail steel. Int J Mech Sci 44:1647-63.

[59] G. Kang, Q. Gao, L. Cai, Y. Sun, (2002). Experimental study on uniaxial and nonproportionally multiaxial ratcheting of SS304 stainless steel at room and high temperatures. Nuclear Engineering and Design 216, 13-26.

[60] Goodman AM (1984). Development of constitutive equations for computer analysis of stainless steel components. Nucl Eng Des 83:349-54.

[61] Das D., Chakraborti P.C. (2011). Effect of stress parameters on ratcheting deformation stages of polycrystalline OFHC copper. Fatigue \& Fracture of Engineering Materials \& Structures, 34, 734-742.

[62] Lim C.-B., Kim K.S., Seong J.B. (2009). Ratcheting and fatigue behavior of a copper alloy under uniaxial cyclic loading with mean stress. International Journal of Fatigue, 31, 501-507.

[63] Paul S.K., Sivaprasad S., Dhar S., Tarafder S. (2010). Ratcheting and low cycle fatigue behavior of SA333 steel and their life prediction. Journal of Nuclear Materials, 401, 17-24.

[64] Gaudin C., Feaugas X. (2004) Cyclic creep process in AISI 316L stainless steel in terms of dislocation patterns and internal stresses. Acta Materialia, 52, 3097-3110.

[65] Jiang Y., Zhang J. (2008). Benchmark experiments and characteristic cyclic plasticity deformation. International Journal of Plasticity, 24, 1481-1515.

[66] Prager W. (1956). A new method of analyzing stresses and strains in work hardening plastic solids. Journal of Applied Mechanics, 23, 493-496.

[67] Bower A.F., Johnson K.L. (1989). The influence of strain hardening on cumulative plastic deformation in rolling and sliding line contact. J. Mech. Phys. Soild, 37, 471-493.

[68] Ohno, N., (1998). Constitutive modeling of cyclic plasticity with emphasis on ratcheting. International Journal of Mechanical Science, 40 (2-3), 397-421. 
[69] Ahmadzadeh, G.R., Hamidinejad, S.M., Varvani-Farahani, A. (2015). Ratcheting Prediction of 1070 and 16MnR Steel Alloys Under Uniaxial Asymmetric Stress Cycles By Means of OhnoWang and Ahmadzadeh-Varvani Kinematic Hardening Rules. J. Pressure Vessel Technol. 137(3), 031001.

[70] Drucker, D. C. (1951). A more fundamental approach to plastic stress strain relations. Proceedings of the First U.S National Congress of Applied, ASME, pp. 487-491.

[71] Drucker, D. C. (1960). Plasticity in structural mechanics. Proceedings of the First (1958) symposium on Naval Structural Mechanics, Goodier and Hoff, Eds., Pergamon, Macmillan, New York, pp. 331-350.

[72] Abdel-Karim M. (2010). An evaluation for several kinematic hardening rules on prediction of multiaxial stress-controlled ratchetting. International Journal of Plasticity 26, 711-730.

[73] Kang GZ, Gao Q, Cai L, Yang X, Sun Y. (2001). Experimental study on uniaxial and multiaxial strain cyclic characteristics and ratcheting of 316L stainless steel. J Mater Sci Technol. 17:219-23.

[74] Ahmadzedehrishehri, Gholamreza, (2013). Modifications on A-F hardening rule to assess ratcheting response of materials and its interaction with fatigue damage under uniaxial stress cycles. PhD thesis, Ryerson University, Toronto, ON, Canada. 